
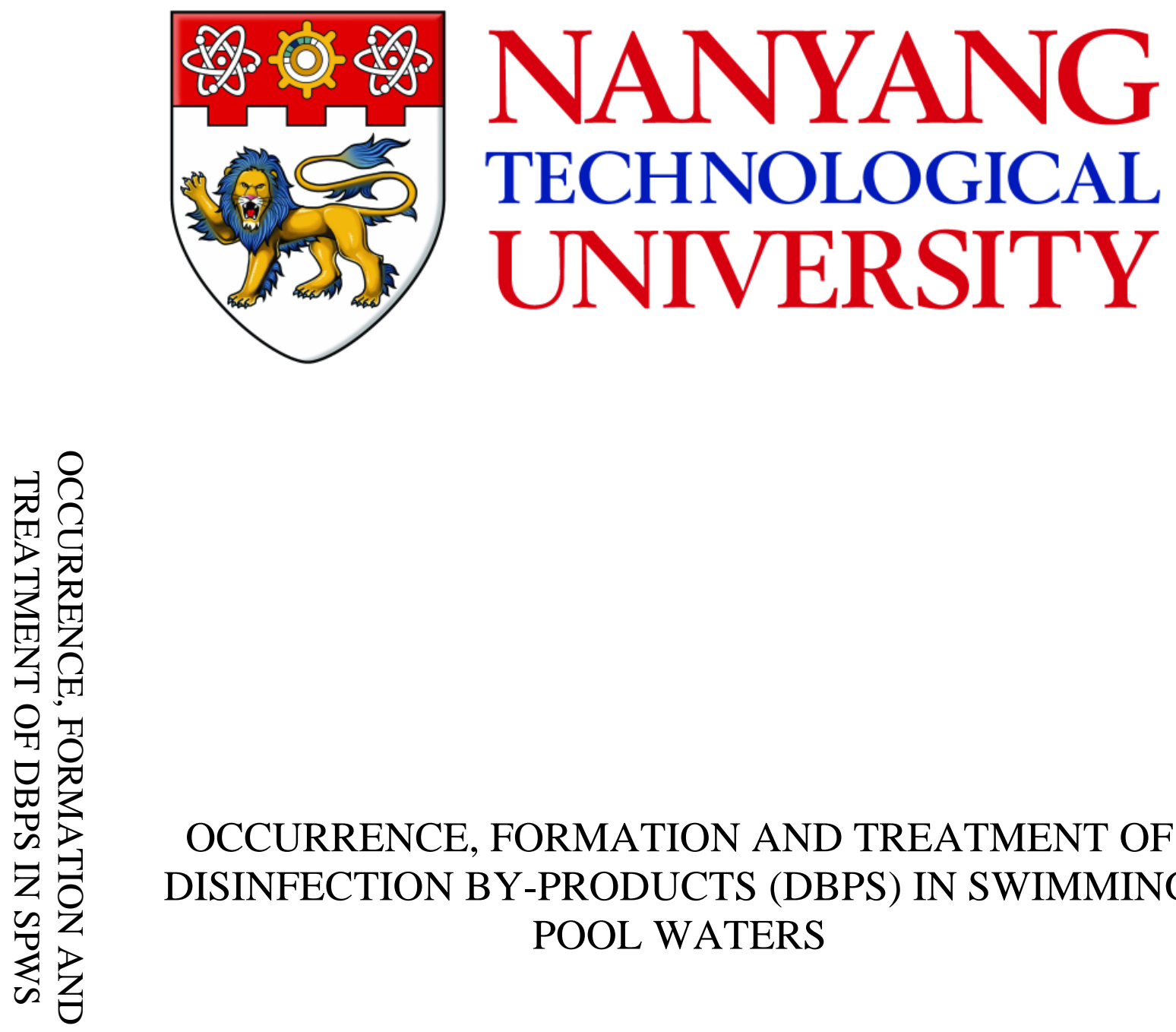

\title{
OCCURRENCE, FORMATION AND TREATMENT OF DISINFECTION BY-PRODUCTS (DBPS) IN SWIMMING POOL WATERS
}

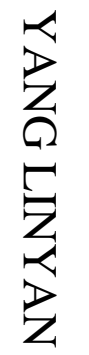

YANG LINYAN

Interdisciplinary Graduate School

Nanyang Environment and Water Research Institute

ำ 


\title{
OCCURRENCE, FORMATION AND TREATMENT OF DISINFECTION BY-PRODUCTS (DBPS) IN SWIMMING POOL WATERS
}

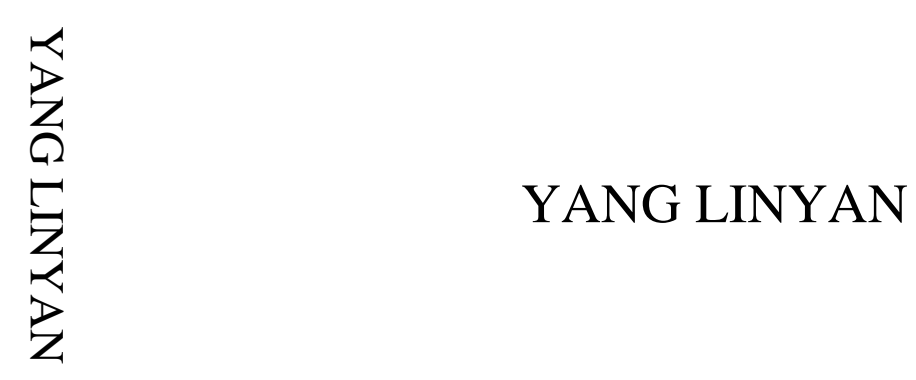

Interdisciplinary Graduate School

Nanyang Environment and Water Research Institute

\author{
A thesis submitted to the Nanyang Technological University in \\ partial fulfilment of the requirement for the degree of \\ Doctor of Philosophy
}




\section{Acknowledgement}

The dissertation was compiled at Interdisciplinary Graduate School (IGS), Nanyang Technological University (NTU), Singapore. I would like to express my sincere gratitude to those who have accompanied and continuously supported me during my $\mathrm{PhD}$ study.

Foremost, I would like to express my deepest gratitude to my main supervisor, Prof. Chang Wei-Chung Victor from School of Civil and Environmental Engineering (CEE), for his invaluable academic guidance in this research and his optimistic attitude towards life which always inspired me. I would also thank my co-supervisor, Prof. Wan Man Pun from School of Mechanical and Aerospace Engineering (MAE), and my mentors, Prof. Wang Jing-Yuan and Prof. Wang Rong from CEE, for their advice and supports. Thanks, Prof. Tang Chuyang from the University of Hong Kong and Prof. Zwiener Christian from Eberhard Karls University Tuebingen, for their patient guidance, great discussions, and feedbacks during my research.

I would also express my thanks to Dr. Schmalz Christina, Dr. She Qianhong and Dr. Ge Liya for sharing their knowledge and laboratory experience (e.g., GCMS, HPLC). I would also acknowledge all my group members, colleagues and friends in IGS, CEE and Nanyang Environment and Water Research Institute (NEWRI) for their great assistance all the way. 
Finally, my special thanks to my husband, Zhou Youjin, not only for his assistance with data analysis but also for his understanding, encouragement, and company throughout my $\mathrm{PhD}$ period. I am also grateful to my parents for their endless love and support. 


\section{Table of Contents}

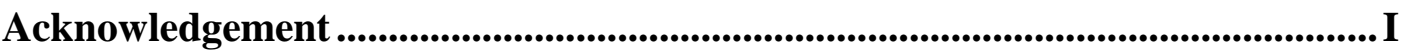

Table of Contents .......................................................................................................III

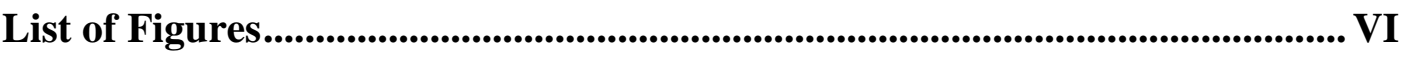

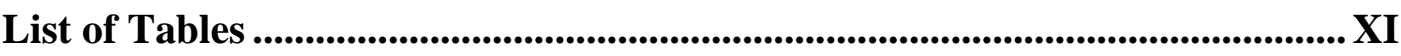

List of Acronyms .......................................................................................................... XIII

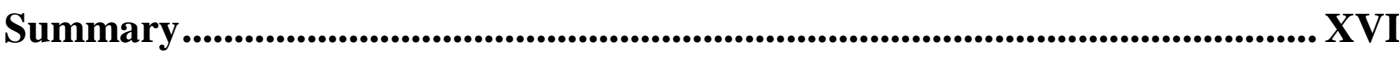

Chapter 1 Introduction.................................................................................1

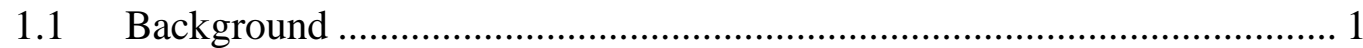

1.2 Problem statement ............................................................................. 3

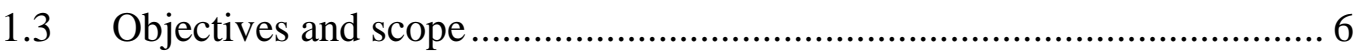

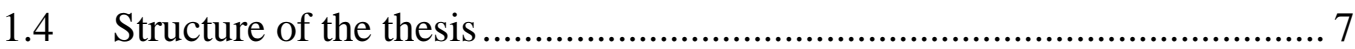

Chapter 2 Literature Review .....................................................................9

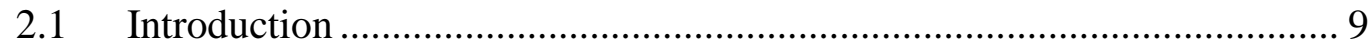

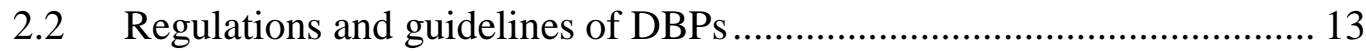

2.3 DBP formation mechanism ............................................................. 15

2.3.1 Disinfectants and their aqueous chemistry ....................................15

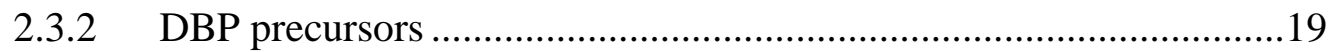

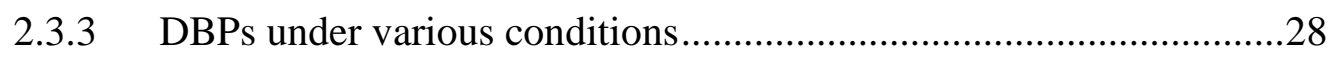

2.4 Toxicity and carcinogenicity of DBPs ............................................. 42

2.5 Human exposure and risk assessment .............................................. 43

2.5.1 Exposure assessment of DBPs by biomarkers ..............................43

2.5.2 Epidemiological evidence and its potential relationship with pool

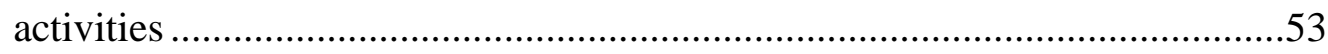

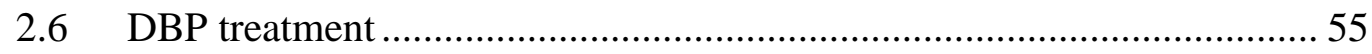

2.6.1 Membrane filtration ...............................................................55

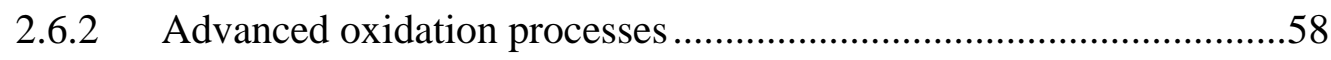

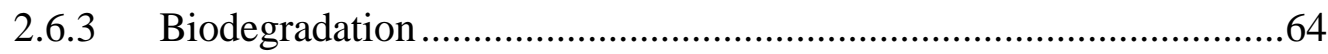

2.6.4 Thermal degradation and chemical reduction..............................65

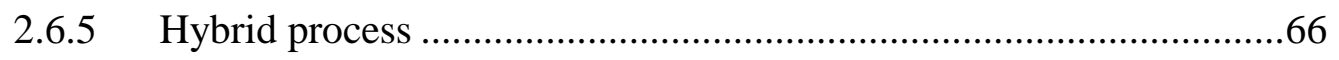


Chapter 3 An Insight of Disinfection By-Product Formation by Alternative Disinfectants for Swimming Pool Disinfection under Tropical Conditions .....70

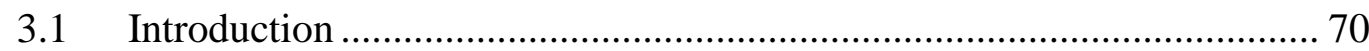

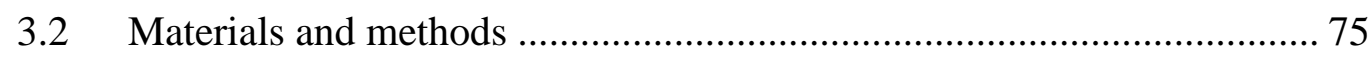

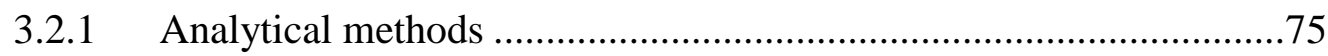

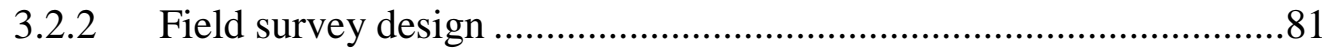

3.2.3 Lab scale batch reactions under simulated swimming pool water

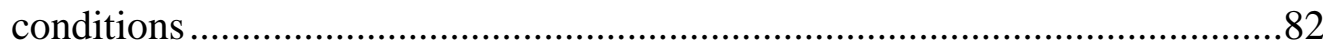

3.2.4 Two-stage models for chlorine decay and DBP formation..................85

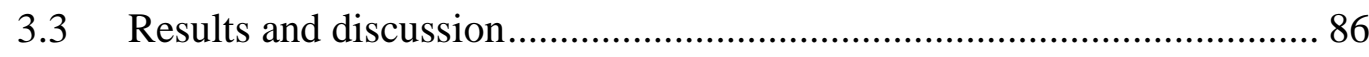

3.3.1 Disinfectant, precursors and DBP occurrence in Singapore pools ....86

3.3.2 Comparison of different disinfectants...................................................89

3.3.3 Effect of reaction time and dosing methods on DBP formation ........92

3.3.4 Effect of temperature on DBP formation ..........................................99

3.3.5 Effect of BFA and free chlorine concentration on DBP formation .101

3.3.6 Comparison of NOM and BFA on DBP formation ..........................104

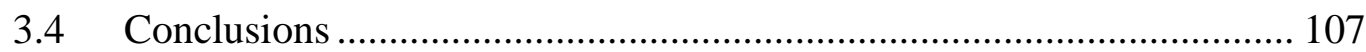

\section{Chapter 4 Removal of Haloacetic Acids from Swimming Pool Water by} Reverse Osmosis and Nanofiltration .........................................................................109

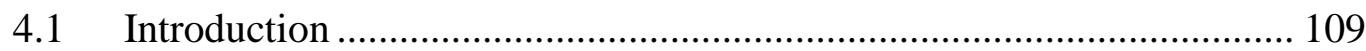

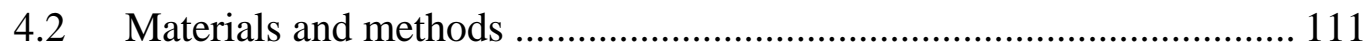

4.2.1 Chemicals and materials ....................................................................111

4.2.2 Membrane characterization ........................................................115

4.2.3 Membrane filtration experiments..................................................116

4.2.4 Analytical methods .........................................................................120

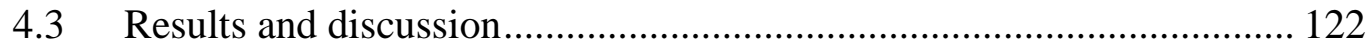

4.3.1 Membrane properties …………………......................................122

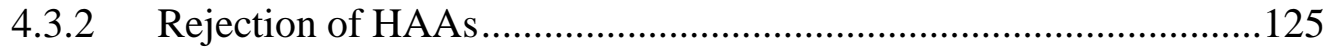

4.3.3 A unified approach for assessing size exclusion and charge repulsion 132

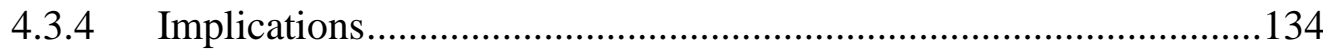




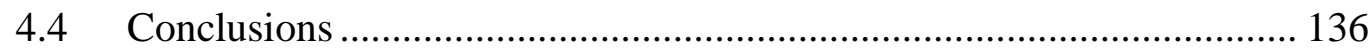

Chapter 5 Role of Calcium Ions on the Removal of Haloacetic Acids from Swimming Pool Water by Nanofiltration: Mechanisms and Implications.....138

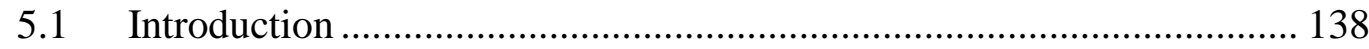

5.2 Materials and methods .................................................................. 140

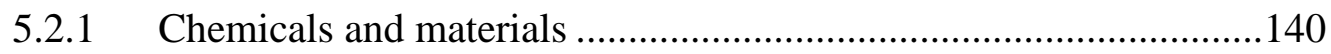

5.2.2 Membrane characterization..........................................................145

5.2.3 Membrane filtration experiments ................................................146

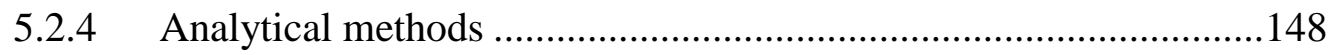

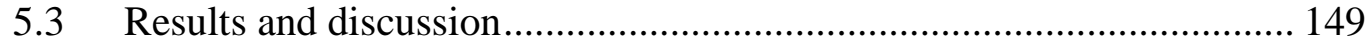

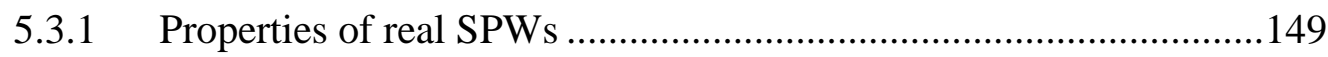

5.3.2 Rejection of inorganic solutes by membranes .............................151

5.3.3 Rejection of HAAs by membranes ............................................154

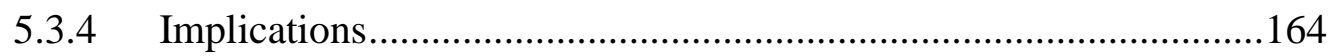

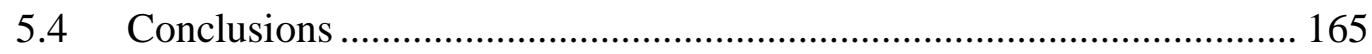

Chapter 6 Conclusions and Recommendations............................................167

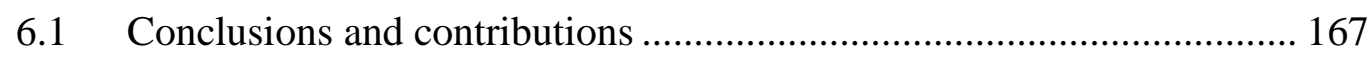

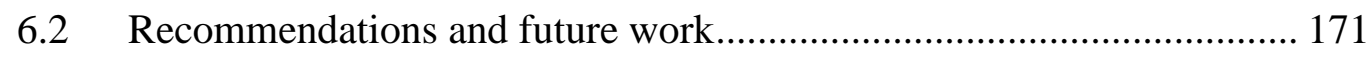

Appendix

A1. Transport model for uncharged solutes .......................................... 174

A2. Transport model for charged ions ................................................. 178

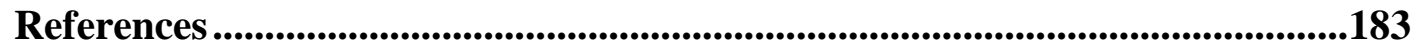

List of Publications ..........................................................................................202 


\section{List of Figures}

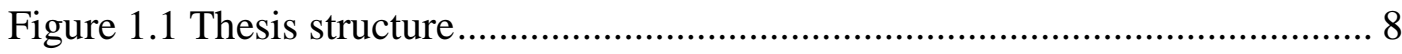

Figure 2.1 The number of publications in relation with DBPs in SPWs and DWs in the past 20 years (1997-2016). Source: Web of Science on June 22, 2016. Keywords for searching: disinfection by-product, swimming pool water/drinking water. 11

Figure 2.2 The relationship between the regulated range of free chlorine and the HAA concentrations in SPWs in different countries. The coordinates of the ellipse vertexes representing the regulated chlorine ranges and reported HAA concentrations. 30

Figure 2.3 The relationship between $\mathrm{CHCl}_{3}$ concentrations in swimming pool water and in ambient air. Other studies include (Catto et al., 2012; Fantuzzi et al., 2001; Fantuzzi et al., 2010; Kogevinas et al., 2010; Lévesque et al., 2000; Lourencetti et al., 2012; Marina et al., 2009; Richardson et al., 2010). 46

Figure 2.4 The relationship between $\mathrm{CHCl}_{3}$ concentrations in ambient air and alveolar air of swimmers. Other studies include (Catto et al., 2012; Fantuzzi et al., 2001; Fantuzzi et al., 2010; Kogevinas et al., 2010; Lévesque et al., 2000; Lourencetti et al., 2012; Marina et al., 2009; Richardson et al., 2010). 48

Figure 2.5 The relationship between $\mathrm{CHCl}_{3}$ concentrations in swimming pool water and in the urine of swimmers after $1 \mathrm{~h}$ swimming and workers after $2 \mathrm{~h}$ work shift. Data are from (Caro and Gallego, 2007; 2008).

Figure 2.6 The relationship between HAA concentrations in SPWs and human urine. Data are from (Cardador and Gallego, 2011; Cardador and Gallego, 2010). 52

Figure 3.1 Flow chart of HAA analysis procedure (adapted from EPA 552.3)..... 77

Figure 3.2 TCCA species before (A) and after (B) the reaction (filling water: tap (1.27 mg/L as TOC); temperature: $30{ }^{\circ} \mathrm{C}$; $\mathrm{pH}: 7.5$; reaction time: 24 
hours; free chlorine: $3 \mathrm{mg} / \mathrm{L}$ as $\mathrm{Cl}_{2}$; $\mathrm{BFA}: 1 \mathrm{mg} / \mathrm{L}$ as TOC; disinfectants: TCCA) 80

Figure 3.3 Free chlorine concentrations at different sampling dates and times in campus pool 1 (empty and shaded columns) are compared to the typical daily variation of the UV index in Singapore (solid line; adapted from NEA at http://www.nea.gov.sg/training-knowledge/weatherclimate/uvradiation-uvindex). The horizontal dashed lines represent the range of free chlorine regulated by NEA Singapore. 88

Figure 3.4 Comparison of three different disinfectants for residual free chlorine/bromine and DBP formation (filling water: tap $(1.27 \mathrm{mg} / \mathrm{L}$ as TOC); temperature: $30{ }^{\circ} \mathrm{C}$; $\mathrm{pH}$ : 7.5 ; reaction time: 24 hours; free chlorine/bromine: $3 \mathrm{mg} / \mathrm{L}$ as $\mathrm{Cl}_{2}$; BFA: $1 \mathrm{mg} / \mathrm{L}$ as TOC; disinfectants: $\mathrm{NaClO} / \mathrm{TCCA} / \mathrm{BCDMH})$ 91

Figure 3.5 Effect of reaction time and dosing methods on residual free chlorine/bromine, formation of bromide ions, and two-stage chlorine decay models (filling water: tap (1.40 mg/L as TOC); temperature: 30 ${ }^{\circ} \mathrm{C}$; pH: 7.5; reaction time: 138 hours; BFA: $1 \mathrm{mg} / \mathrm{L}$ as TOC; disinfectants: $\mathrm{NaClO} / \mathrm{TCCA} / \mathrm{BCDMH}$ ) 93

Figure 3.6 Effect of reaction time and dosing methods on THM and HAA formation, and two-stage DBP formation models (filling water: tap $(1.40 \mathrm{mg} / \mathrm{L}$ as TOC); temperature: $30{ }^{\circ} \mathrm{C}$; $\mathrm{pH}$ : 7.5 ; reaction time: 138 hours; BFA: 1 $\mathrm{mg} / \mathrm{L}$ as TOC; disinfectants: $\mathrm{NaClO} / \mathrm{TCCA} / \mathrm{BCDMH})$ 96

Figure 3.7 Effect of temperature on DBP formation (filling water: tap $(1.14 \mathrm{mg} / \mathrm{L}$ as TOC); temperature: $25,30,35$ and $40{ }^{\circ} \mathrm{C} ; \mathrm{pH}$ : 7.5; reaction time: 24 hours; free chlorine/bromine: $3 \mathrm{mg} / \mathrm{L}$ as $\mathrm{Cl}_{2}$; $\mathrm{BFA}: 1 \mathrm{mg} / \mathrm{L}$ as TOC; disinfectants: $\mathrm{NaClO} / \mathrm{TCCA} / \mathrm{BCDMH}$ ) 101

Figure 3.8 Effect of temperature on residual free chlorine/bromine (filling water: tap (1.14 mg/L as TOC); temperature: $25,30,35$ and $40{ }^{\circ} \mathrm{C} ; \mathrm{pH}: 7.5$; reaction time: 24 hours; free chlorine/bromine: $3 \mathrm{mg} / \mathrm{L}$ as $\mathrm{Cl}_{2}$; BFA: $1 \mathrm{mg} / \mathrm{L}$ as TOC; disinfectants: $\mathrm{NaClO} / \mathrm{TCCA} / \mathrm{BCDMH})$ 101 
Figure 3.9 Effect of BFA and free chlorine/bromine concentration on THM and HAA formation (filling water: tap $\left(1.27 \mathrm{mg} / \mathrm{L}\right.$ as TOC); temperature: $30{ }^{\circ} \mathrm{C}$; pH: 7.5; free chlorine/bromine: 1 or $3 \mathrm{mg} / \mathrm{L}$ as $\mathrm{Cl}_{2}$; BFA: 0 or 1 or 5 $\mathrm{mg} / \mathrm{L}$ as TOC; reaction time: 24 hours; disinfectants:

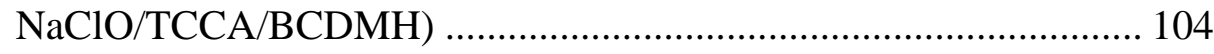

Figure 3.10 Effect of NOM and BFA on DBP formation (temperature: $30^{\circ} \mathrm{C}$; $\mathrm{pH}$ : 7.5, reaction time: 24 hours; free chlorine/bromine: $3 \mathrm{mg} / \mathrm{L}$ as $\mathrm{Cl}_{2}$; TOC: $1 \mathrm{mg} / \mathrm{L}$; disinfectants: $\mathrm{NaClO} / \mathrm{TCCA} / \mathrm{BCDMH})$........................... 106

Figure 3.11 DBP formation potential of individual component in BFA (filling water: DI; temperature: $30{ }^{\circ} \mathrm{C} ; \mathrm{pH}: 7.5$, reaction time: 24 hours; free chlorine: 3 $\mathrm{mg} / \mathrm{L}$ as $\mathrm{Cl}_{2}$; ammonium chloride as $\mathrm{TN}$ and other individual component as TOC: $1 \mathrm{mg} / \mathrm{L}$; disinfectants: $\mathrm{NaClO}$ ) ...................................... 106

Figure 4.1 Schematic diagram of the membrane filtration setup....................... 116

Figure 4.2 Chromatogram of a standard with a concentration level of $200 \mathrm{mg} / \mathrm{L}$ by HPLC-RID.

Figure 4.3 SEM images of the membrane rejection layers. Micrographs were obtained at an accelerating voltage of $2 \mathrm{keV}$. The scale bar is $100 \mathrm{~nm}$. 123

Figure 4.4 FTIR, zeta potential and MWCO of the four membranes. A: FTIR spectra of virgin membranes. B: Zeta potential of virgin membranes (in a 0.05 $\mathrm{M} \mathrm{NaCl}$ background electrolyte over $\mathrm{pH} \sim 2-11)$. C: MWCOs of the membranes obtained from the surrogate rejection tests (experimental conditions: $100 \mathrm{psi}, \mathrm{pH} \sim 6.7,25^{\circ} \mathrm{C}$, feed water containing $200 \mathrm{mg} / \mathrm{L}$ of each surrogate). 124

Figure 4.5 Effect of the molecular weight on rejection. Experimental conditions: 100 psi, pH 3.5, $25{ }^{\circ} \mathrm{C}$, feed water containing $100 \mu \mathrm{g} / \mathrm{L}$ of each HAA and $0.05 \mathrm{M} \mathrm{NaCl}$ for HAA rejection tests or $200 \mathrm{mg} / \mathrm{L}$ of each surrogate for surrogate rejection tests. Vertical dash lines represent the membrane MWCOs. 126

Figure 4.6 Effect of the molecular radius on rejection. Experimental conditions: 100 psi, $\mathrm{pH} 3.5,25^{\circ} \mathrm{C}$, feed water containing $100 \mu \mathrm{g} / \mathrm{L}$ of each HAA and 
$0.05 \mathrm{M} \mathrm{NaCl}$ for HAA rejection tests or $200 \mathrm{mg} / \mathrm{L}$ of each surrogate for surrogate rejection tests. Vertical dash lines represent the membrane pore radii. 128

Figure 4.7 Effect of $\mathrm{pH}$ on rejections of surrogates (A) and HAAs (B) for NF270 membrane. Experimental conditions: 100 psi, pH over 3.5 to $7.5,25{ }^{\circ} \mathrm{C}$, feed water containing $100 \mu \mathrm{g} / \mathrm{L}$ of each $\mathrm{HAA}$ and $0.05 \mathrm{M} \mathrm{NaCl}$ for HAA rejection tests or $200 \mathrm{mg} / \mathrm{L}$ of each surrogate for surrogate rejection tests.

Figure 4.8 Effect of $\mathrm{pH}$ on rejections of surrogates and HAAs for XLE, NF90 and SB50 membranes. Experimental conditions: 100 psi, pH over 3.5 to 7.5, $25^{\circ} \mathrm{C}$, feed water containing $100 \mu \mathrm{g} / \mathrm{L}$ of each $\mathrm{HAA}$ and $0.05 \mathrm{M} \mathrm{NaCl}$ for HAA rejection tests or $200 \mathrm{mg} / \mathrm{L}$ of each surrogate for surrogate rejection tests.

Figure 4.9 Normalized rejection evaluation. A. Normalized rejection as a function of $\lambda$ at $\mathrm{pH}$ 3.5. B. Normalized rejection as a function of $\lambda$ at $\mathrm{pH}$ 7.5. C. Normalized rejection as a function of $\lambda_{\mathrm{eff}}$ at $\mathrm{pH}$ 7.5. Vertical dash lines represent a critical point where $\lambda$ or $\lambda_{\text {eff }}=1$. The effective hindrance parameter $\lambda_{\text {eff }}\left(=r_{\text {eff }} / r_{p}\right)$ is calculated based on an effective molecular radius $r_{\text {eff }}$ given by $r_{s}+0.045 \cdot \Lambda_{d}$

Figure 5.1 Urea calibration curve (A) and the calibration standard illustration (B)

Figure 5.2 The rejection of components in real and synthetic SPWs by NF270 and NF90. 152

Figure 5.3 Effect of calcium ions on rejections of neutral compounds, cations, and anions by NF90 and NF270 .... 154

Figure 5.4 Effect of calcium ions on HAA rejection. Rejection data for NF270 (A) and NF90 (B) using synthetic SPW at pH 7.5. A conceptual diagram (C) illustrates the three possible mechanisms involved (1. HAA-Ca ${ }^{2+}$ induced effect; 2. membrane- $\mathrm{Ca}^{2+}$ induced size exclusion effect; and 3 membrane-Ca ${ }^{2+}$ induced charge interaction effect). 156 
Figure 5.5 Species distribution of chloroacetic acid, bromoacetic acid, and acetic acid in the presence of calcium ions. The log values of stability constants for calcium-based complexes are $0.14,0.55$, and 1.18 , respectively. 157

Figure 5.6 Effect of calcium ions on surrogate rejection by NF270 (A) and NF90 (B), and membrane pore size (C) calculated in accordance to Nghiem et al. (2004).

Figure 5.7 Effect of calcium ions on water flux (for surrogate filtration tests) .... 159 Figure 5.8 Effect of calcium ions on zeta potential for NF90 and NF270 (A). High resolution XPS spectra (duplicate analysis) for NF270 exposed in $\mathrm{Ca}^{2+}$ solutions at different concentrations of $0 \mathrm{mM}$ as control (B), $0.1 \mathrm{mM}$ (C), and $1 \mathrm{mM}$ (D). The minor Ca peak for the control membrane was caused by the $0.001 \mathrm{mM} \mathrm{CaCl}_{2}$ rinse solution....................................... 161

Figure 5.9 HAA rejection as a function of molecular radius at $\mathrm{pH} 3.5$ and 7.5 in the presence of calcium ions by NF270 ............................................ 162

Figure 5.10 Effect of calcium ions on HAA rejection at $\mathrm{pH} 3.5$ by NF270. The dash lines represent the average rejection under various concentrations of

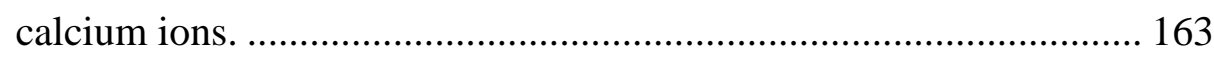

Figure 5.11 Comparison of measured and simulated conductivity rejection in the presence of calcium ions ........................................................... 165

Figure 6.1 Proposed treatment process for SPWs ...................................... 173 


\section{List of Tables}

Table 2.1 DBP regulations and guidelines in drinking water ............................. 14

Table 2.2 DBP regulations and guidelines in SPWs .......................................... 15

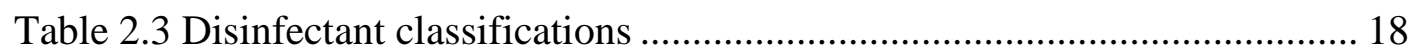

Table 2.4 Regulated conditions for the operation of swimming pools .................. 38

Table 2.5 THM concentrations in SPWs ........................................................... 39

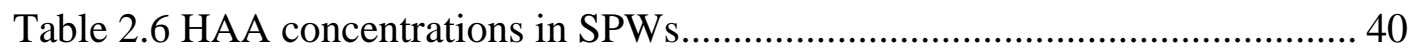

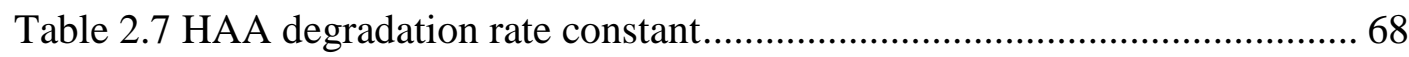

Table 3.1 THM method development and validation parameters with retention time, quantifier and qualifier ions, linearity, repeatability and reproducibility ${ }^{\mathrm{a}}$ 78

Table 3.2 HAA method development and validation parameters with retention time, quantifier and qualifier ions, linearity, repeatability and reproducibility ${ }^{\mathrm{a}}$

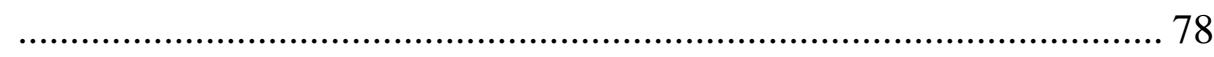

Table 3.3 Tap water characteristics and BFA components................................ 83

Table 3.4 Disinfectants, precursors and DBPs in different swimming pools in

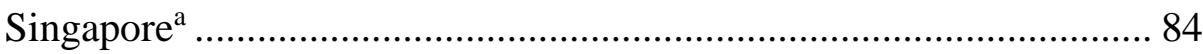

Table 3.5 An overview of all batch experimental conditions ............................. 84

Table 3.6 Formation of THMs by three disinfectants (filling water: tap $(1.27 \mathrm{mg} / \mathrm{L}$ as TOC); temperature: $30{ }^{\circ} \mathrm{C} ; \mathrm{pH}$ : 7.5 ; reaction time: 24 hours; free chlorine/bromine: $3 \mathrm{mg} / \mathrm{L}$ as $\mathrm{Cl}_{2}$; BFA: $1 \mathrm{mg} / \mathrm{L}$ as TOC; disinfectants:

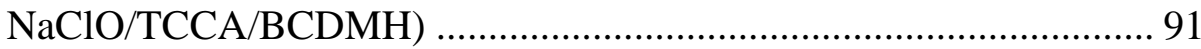

Table 3.7 Formation of HAAs by three disinfectants (filling water: tap (1.27 mg/L as TOC); temperature: $30{ }^{\circ} \mathrm{C}$; $\mathrm{pH}: 7.5$; reaction time: 24 hours; free chlorine/bromine: $3 \mathrm{mg} / \mathrm{L}$ as $\mathrm{Cl}_{2}$; BFA: $1 \mathrm{mg} / \mathrm{L}$ as TOC; disinfectants: $\mathrm{NaClO} / \mathrm{TCCA} / \mathrm{BCDMH})$......................................................... 92

Table 3.8 Comparison of NOM and BFA on DBP formation (temperature: $30{ }^{\circ} \mathrm{C}$; $\mathrm{pH}$ : 7.5, reaction time: 24 hours; free chlorine/bromine: $3 \mathrm{mg} / \mathrm{L}$ as $\mathrm{Cl}_{2}$; NOM or BFA: $1 \mathrm{mg} / \mathrm{L}$ as TOC; disinfectants: $\mathrm{NaClO} / \mathrm{TCCA} / \mathrm{BCDMH}$ ) 
Table 4.1 Haloacetic acid and surrogate properties ....................................... 112

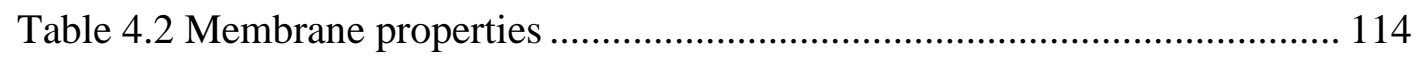

Table 4.3 Membrane pore radius estimation.................................................... 119

Table 5.1 Properties of HAAs and surrogate molecules................................... 141

Table 5.2 Recipe for the preparation of synthetic SPW ${ }^{\mathrm{a}}$.................................. 142

Table 5.3 Membrane properties (Data were extracted from Table 4.2, except RMS roughness from Tang et al. (2009b) and contact angle by this study) 144

Table 5.4 Characteristics of swimming pool water ....................................... 150 


\section{List of Acronyms}

$\begin{array}{ll}\text { ANSES } & \text { French Agency for Food, Environmental and } \\ & \text { Occupational Health \& Safety } \\ \text { AOP } & \text { Advanced Oxidation Process } \\ \text { AOX } & \text { Adsorbable Organic Halides } \\ \text { ATR-FTIR } & \text { Attenuated Total Reflection Fourier Transform } \\ & \text { Infrared Spectroscopy } \\ \text { BCDMH } & \text { Bromochlorodimethylhydantoin } \\ \text { BDCAA } & \text { Bromodichloroacetic Acid } \\ \text { BFA } & \text { Body Fluid Analog } \\ \text { Br-DBP, HAA, THM } & \text { Brominated Disinfection By-Product, Haloacetic } \\ & \text { Acid, Trihalomethane } \\ \text { C-, N-DBP } & \text { Carbonaceous, Nitrogenous Disinfection By-Product } \\ \text { CDC } & \text { Centres for Disease Control and Prevention } \\ \text { Cl }{ }_{2} \text { Cy } & \text { Dichloroisocyanurate Ion } \\ \text { Cl-DBP, HAA, THM } & \text { Chlorinated Disinfection By-Product, Haloacetic } \\ & \text { Acid, Trihalomethane } \\ \text { DBP } & \text { Disinfection By-Product } \\ \text { DCCA } & \text { Dichloroisocyanuric Acid } \\ \text { DOC } & \text { Dissolved Organic Carbon } \\ \text { DPD } & \text { Dipropyl-P-Phenylenediamine } \\ \text { DW } & \text { Drinking Water } \\ \text { EGMO } & \text { Electrochemically Generated Mixed Oxidant } \\ \text { FE-SEM } & \text { Field Emission Scanning Electron Microscopy } \\ \text { FP } & \text { Formation Potential } \\ \text { HAA } & \text { Haloacetic Acid } \\ \text { HAN } & \text { Haloacetonitrile } \\ \text { HClCy } & \text { Monochloroisocyanurate Ion } \\ \text { HNM } & \text { Halonitromethane } \\ & \end{array}$


HPLC-RID

HS-GCMS

HS

IARC

ICP-MS

ICP-OES

I-DBP, HAA, THM

LOQ

MCAA, MBAA,

DCAA, TCAA, BCAA DBAA, BDCAA,

DBCAA, TBAA

MCL

M-, D-, T-DBP

M-, D-, T-HAA

MTBE

MW

MWCO

NEA

NOM

NSPF

PCP

PEG

PPCP

RO, NF, UF, FO
High-Performance Liquid Chromatography Coupled with Refractive Index Detector Headspace Gas Chromatography - Mass Spectrometry Helmholtz-Smoluchowski

International Agency for Research on Cancer Inductively Coupled Plasma - Mass Spectroscopy Inductively Coupled Plasma - Optical Emission Spectrometry

Iodinated Disinfection By-Product, Haloacetic Acid, Trihalomethane

Limit of Quantification

Chloroacetic, Bromoacetic,

Dichloroacetic, Trichloroacetic, Bromochloroacetic, Dibromoacetic, Bromodichloroacetic, Dibromochloroacetic, Tribromoacetic Acid Maximum Contaminant Level

Monohalogenated, Dihalogenated, Trihalogenated Disinfection By-Product

Monohalogenated, Dihalogenated, Trihalogenated

Haloacetic Acid

Methyl Tert-Butyl Ether

Molecular Weight

Molecular Weight Cut-Off

National Environment Agency

Natural Organic Matter

National Swimming Pool Foundation

Personal Care Product

Polyethylene Glycol

Pharmaceuticals and Personal Care Product

Reverse Osmosis, Nanofiltration, Ultrafiltration, Forward Osmosis 
RSD

SE

SPW

TCCA

tHAA, tTHM

THM

TN

TOC

TOX

U.S. EPA

UV-US

WHO

XPS
Relative Standard Deviation

Size Exclusion

Swimming Pool Water

Trichloroisocyanutic Acid

Sum of Nine Haloacetic Acids, Four Trihalomethanes

Trihalomethane

Total Nitrogen

Total Organic Carbon

Total Organic Halides

United States Environmental Protection Agency

UV Radiation and Ultrasound Sonolysis

World Health Organization

X-Ray Photoelectron Spectroscopy 


\section{Summary}

With the popularity of swimming activities across the world, the responsibility of the pool operators and managers to ensure the microbial and chemical safety of the pool participants becomes extremely significant. Disinfection by-products (DBPs) in swimming pool waters (SPWs) have attracted considerable public attentions due to their high frequency of occurrence, considerable concentrations and potent toxicity. This study explored the effectiveness and appropriateness of using alternative disinfectants to minimize the formation of DBPs from the source and using membrane technology to remove the already generated haloacetic acids (HAAs, a group of prevalent DBPs) from the simulated SPWs.

Firstly, we reviewed the latest research progress on the occurrence, formation, and treatment of DBPs in the context of SPWs. More specifically, it provided comprehensive knowledge and information from multiple aspects, e.g., DBP regulations in different regions, formation mechanisms related with different disinfectants, precursors and other factors, toxicity and carcinogenicity, human exposure assessment reflected by biomarkers or epidemiological evidence, and latest approaches for the control and treatment of DBPs. This critical review recommended the potential directions of minimizing the DBP formation and handling the formed DBPs for the purpose of DBP control. It demonstrated that chlorine/bromine stabilized disinfectants carry the potential to sustain the sufficient effective disinfection species in pools, especially for those exposed under sunlight. However, the formation of DBPs from these alternative disinfectants is still unclear. In addition, 
membrane filtration turned out to be a promising approach to control DBPs and DBP precursors. The underlying mechanisms behind have not been comprehensively understood and most laboratory-scale investigations ignored many significant realistic conditions. These unknown and unclear situations triggered our curiosities and passions for further investigations.

Secondly, two alternative organic-based disinfectants, trichloroisocyanuric acid (TCCA, $\mathrm{C}_{3} \mathrm{Cl}_{3} \mathrm{~N}_{3} \mathrm{O}_{3}$ ) and bromochlorodimethylhydantoin (BCDMH, $\mathrm{C}_{5} \mathrm{H}_{6} \mathrm{BrClN}_{2} \mathrm{O}_{2}$ ), were investigated and compared to $\mathrm{NaClO}$ (a most commonly used disinfectant in pool treatment system) in terms of its self-degradation and the formation of DBPs, including trihalomethanes (THMs) and HAAs, under simulated tropical climate conditions. The motivations of this study include that pools in tropical regions usually suffer from the strong sunlight irradiation which degrades the free chlorine rapidly, and more pools start to adopt the recirculation of SPWs, which intensifies the DBP accumulation issue. The result revealed that halogen stabilizer, TCCA, has the advantages of slower free chlorine degradation and lower DBP concentration compared to $\mathrm{NaClO}$, and thus makes it a good alternative disinfectant. BCDMH was not recommended mainly due to the highly reactive disinfecting ingredient, hypobromous acid, which fails to sustain the continuous disinfection requirement. Total disinfectant dosage was the main factor that affects residual chlorine and THM/HAA formation regardless of different disinfectant dosing methods, e.g. shock dosing (one-time spiking) in the beginning and continuous dosing during the whole period. Two-stage second-order-kinetic-based 
models demonstrate good correlations between the measured and predicted data for chlorine decay $\left(R^{2} \geqslant 0.95\right)$, THM $\left(R^{2} \geqslant 0.99\right)$ and HAA $\left(R^{2} \geqslant 0.83\right)$ formation. Higher temperature was found to enhance the DBP formation due to the temperature dependence of reaction rates. Thus, temperature control of pools, especially for those preferring higher temperatures (e.g. hydrotherapy and spa), should take both bather comfort and DBP formation potential into consideration. It is also observed that chlorine competition existed between different precursors from natural organic matters (NOM) in filling water and body fluids from pool users. Among the composition of human body fluids, uric acid, citric acid and hippuric acid were found as main precursors of HAA formation.

Thirdly, we investigated the removal of 9 HAAs (a prevalent class of toxic DBPs) by four commercial reverse osmosis (RO) and nanofiltration (NF) membranes. Under typical SPW conditions (pH 7.5 and $50 \mathrm{mM}$ ionic strength), HAA rejections were $>60 \%$ for loose NF270 membrane and $\sim$ or $>90 \%$ for tighter membranes (XLE, NF90 and SB50) as a result of the combined effects of size exclusion and charge repulsion. We further included 7 neutral hydrophilic surrogates as molecular probes to resolve the rejection mechanisms. In the absence of strong electrostatic interaction (e.g., $\mathrm{pH}$ 3.5), the rejection data of HAAs and surrogates by various membranes fall onto an identical size-exclusion (SE) curve when plotted against the hindrance parameter, i.e., the ratio of molecular radius over membrane pore radius. The independence of this SE curve on molecular structures and membrane properties reveals that the hindrance parameter is a more fundamental SE descriptor compared 
to molecular weight. An effective molecular size can be further used to take account for charge repulsion in addition to size exclusion. The current study provides valuable insights on the rejection of trace contaminants by RO/NF membranes.

Fourthly, the strong matrix effect (particularly by $\mathrm{Ca}^{2+}$ ) on membrane rejection prompts us to systematically investigate the mechanistic role of $\mathrm{Ca}^{2+}$ in HAA rejection. Two nanofiltration membranes NF270 and NF90 have been investigated in this study. At typical SPW pH of 7.5, NF90 maintained consistently high rejection of HAAs (> 95\%) with little influence by $\mathrm{Ca}^{2+}$, thanks to the dominance of size exclusion effect for this tight membrane (pore radius $\sim 0.31 \mathrm{~nm}$ ). In contrast, the rejections of both inorganic ions (e.g., $\mathrm{Na}^{+}$and $\mathrm{Cl}^{-}$) and $\mathrm{HAA}$ anions were decreased at higher $\mathrm{Ca}^{2+}$ concentration for NF270 (pore radius $\sim 0.40 \mathrm{~nm}$ ). Further tests show that the rejection of neutral hydrophilic molecular probes and the membrane pore size were not affected by $\mathrm{Ca}^{2+}$. Although $\mathrm{Ca}^{2+}$ is unable to form a strong complex with HAAs, we observed the binding of $\mathrm{Ca}^{2+}$ to NF270 together with a reduction in its surface charge. Therefore, the formation of membrane- $\mathrm{Ca}^{2+}$ complex, which weakens charge interaction effect, was responsible for the reduced HAA rejection. The current study reveals important mechanistic insights of the matrix effect on trace contaminant rejection, which is critical for a better understanding of their fate and removal in membrane-based treatment. 


\section{Chapter 1 Introduction}

\section{$1.1 \quad$ Background}

With the popularity of swimming activities across the world, the responsibility of the pool operators and managers to ensure the microbial and chemical safety of the pool participants becomes extremely significant. Disinfection plays a predominant role to guarantee microbiologically safe and hygienic swimming pool waters (SPWs). Nevertheless, disinfectants also react with water constituents of natural and anthropogenic origin to yield disinfection by-products (DBPs) (Fischer et al., 2012). Scientists started to be aware of DBPs since the early 1970s and first regulation related to DBPs (including four trihalomethanes (THMs), i.e., chloroform, bromodichloromethane, dibromochloromethane, and bromoform) in the context of drinking water (DW) was issued in 1979 by United States Environmental Protection Agency (U.S. EPA) (EPA, 1979). In 1998, the regulation was upgraded and included seven more species, i.e., five haloacetic acids (HAAs), bromate, and chlorite (EPA, 1998). Among these, HAAs as a group of prevalent DBPs were regulated with a maximum contaminant level (MCL) of $60 \mu \mathrm{g} / \mathrm{L}$ for the sum of five species. In addition, the MCL for four THMs lowered from 100 to $80 \mu \mathrm{g} / \mathrm{L}$ (EPA, 1998). In recent years, some developed European countries (e.g., Germany, Switzerland) have just started to regulate THMs, as a group of most notable DBPs, with MCLs in a range of 20$100 \mu \mathrm{g} / \mathrm{L}$ in the context of SPWs. However, a so-far comprehensive field survey showed the occurrence of more than 100 DBPs in public swimming pools (Richardson et al., 2010). These DBPs include THMs, HAAs, haloacids, halodiacids, haloaldehydes, halonitriles, haloketones, halonitromethanes 
(HNMs), haloamides, haloalcohols, and other halogenated and non-halogenated DBPs, many of which have yet been reported in SPWs and are even not exist in the current mass spectral library database. In addition, DBP concentrations in real SPWs have a high frequency of exceeding the regulated values for SPWs or sometimes are even more than one or two orders of magnitude higher than the regulated values for DWs.

Toxicological studies show the potentially cytotoxic, genotoxic, mutagenic, teratogenic or even carcinogenic properties of DBPs to a variety of cells and animal species (Dad et al., 2013; Plewa et al., 2010; Procházka et al., 2015; Richardson et al., 2007). U.S. EPA or International Agency for Research on Cancer (IARC) have assessed six DBPs, including 4 THMs, dichloroacetic acid and bromate, as probable or possible human carcinogens. Nevertheless, many unregulated or un-carcinogen characterized DBPs may be more toxic although the lack of available toxicology data. In general, nitrogenous, brominated, and iodinated DBPs (N-, Br-, I-DBPs) are more toxic than chlorinated and carbonaceous ones (Cl-, C-DBPs) (Muellner et al., 2007b; Richardson et al., 2007). In addition, extracts of SPWs showed genotoxic effects in Ames-Test, with HepG2 cells, with CHO cells in a comet assay (Glauner et al., 2005b; Liviac et al., 2010; Richardson, 2010).

There are three main exposure routes of DBPs into human bodies: 1) inhalation of volatile compounds or aerosolized solutes; 2) dermal absorption via skin; 3) ingestion of DBP-contaminated water. In the early 2000s, researchers have started to assess DBP exposure via measuring the DBP concentrations in 
swimmers' body matrix, including exhaled air, urine, blood, plasma, etc., as biomarkers (Cardador and Gallego, 2011; Cardador and Gallego, 2010; Caro and Gallego, 2007; 2008; Erdinger et al., 2004; Nieuwenhuijsen et al., 2000). The DBPs detected in exhaled air reached dozens to hundreds of $\mu \mathrm{g} / \mathrm{m}^{3}$ levels and in urine at single digital $\mu \mathrm{g} / \mathrm{L}$ levels for bathers or pool workers. Some diseases and uncomfortable symptoms, such as bladder cancer, ocular, cutaneous, asthmarelated symptoms, and other respiratory allergies or airways inflammation, have been reported by pool participants (Bernard et al., 2008; Fantuzzi et al., 2010; Villanueva and Font Ribera, 2012; Villanueva et al., 2007a). The finding that the total uptake of THMs by pregnant women from swimming (23\%) was far more than that from DWs (1\%) challenged the public-recognized opinion that swimming is a highly recommended activity during pregnancy (Villanueva et al., 2007b). Hence, the chemical safety of SPWs is attracting more attention from the researchers or even the public in recent years.

\subsection{Problem statement}

THMs and HAAs are two most prevalent groups of DBPs due to their high frequency of occurrence, considerable concentrations and potent toxicity. Toxicity effect of THMs is associated with diseases such as bladder and colon cancer, asthma, irritation to the eyes and mucous membrane, and reproductive function (Aggazzotti et al., 2004; Erdinger et al., 1998; Goodman and Hays, 2008; Hamidin et al., 2008). HAAs are mutagenic in bacteria and induce DNA damage and chromosomal aberrations in mammalian cells in vitro (IARC, 2004; Richardson et al., 2007). Besides, some HAAs cause liver tumors, leukemias and abdominal cavity mesotheliomas in experimental animals (Richardson et al., 
2007). As mentioned, the first government department U.S. EPA has devoted to formulating the DBP-related regulation and it stipulated THMs and HAAs with MCLs of 80 and $60 \mu \mathrm{g} / \mathrm{L}$, respectively, in DWs (EPA, 1998). However, after reviewing DBPs in real SPWs in existing literature, we found that the concentrations of DBPs, HAAs in particular, were extremely high. It is surprising but not accidental to observe HAA concentrations with thousands of $\mu \mathrm{g} / \mathrm{L}$ levels (Kanan et al., 2015; Loos and Barceló, 2001; Simard et al., 2013; Wang et al., 2014; Yeh et al., 2014). The high concentration of DBPs, particularly HAAs, in SPWs could be attributed to the following reasons.

1) Abundant reactants. A large amount of organic matters with various species (natural organic matter (NOM), body fluid and personal care products (PCPs), with single- or two-digital mg/L levels) are from source water or from the continual release from swimmers. It is also a common practice to dose disinfectants continuously for SPW treatment. Abundant organic matters and disinfectants are reacted to produce substantial DBPs.

2) Contaminant accumulation. The fate of SPWs normally follows "pool watertreatment-pool water" for the purpose of water and chemical saving. A study shows only about $1 \%$ of fresh water is added to the pools to offset the daily water consumption via evaporation and other mechanisms. The retention time of SPWs reaches as high as 100 days (Arnaud, 2016). Hence more and more contaminants are accumulated in the pool system with time.

3) Highly persistent characteristic. Some DBPs are thermally and chemically 
stable, resistant to biodegradation and therefore, extremely persistent in SPW environment. For example, HAAs are polar and non-volatile, leading to a long residence time in the aqueous phase. The specific SPW matrix, i.e., consistent high chlorine concentration, could be a scourge that deactivates the corresponding bio-degraders and results in invalid DBP biodegradation (Chuang et al., 2011).

The high DBP concentration in SPWs demonstrates the traditional SPW treatment system (e.g., flocculation-sand filtration-chlorination, mainly for the removal of conventional pollutants) seems inadequate to meet the current water quality requirement. With the strict regulation for halogenated DBPs, some halogen-free disinfectants, e.g., $\mathrm{UV}, \mathrm{O}_{3}$, chloramines, etc., have been attempted. Although THMs and HAAs may be eliminated, the changes of disinfection practice can cause new issues, e.g., unable to sustain long time disinfection ability, formation of new kinds of DBPs with unknown or higher toxicity (Richardson et al., 2007). Hence, we are looking for alternative disinfectants with more reliable properties and without generating new issues. In additions, recent research progress demonstrated the potential of some DBP treatment methods, e.g., membrane filtration, biodegradation, etc., in laboratory-, or at most pilot-scale experiments. However, mechanistic understanding to these studies always remains insufficient. To date, the launched SPW treatment process specifically targeting at DBP removal is scarce. In addition, there is a lack of systematic investigation mainly aiming at HAA removal in the context of SPWs. Hence, there is an urgent need to explore novel and effective approaches in a more systematic pattern to minimize the DBP formation or to purify the formed DBPs 
in SPWs. The fundamental understanding of its mechanism would be of great significance to full-scale applications in the future.

\subsection{Objectives and scope}

The necessity and the significance of this research is reflected by the current DBP-related properties and issues in SPW context, i.e., high DBP concentration in SPWs, toxic and carcinogenic properties of some DBPs, considerable DBPs detected in the body matrix of pool participants, epidemiological evidence related with pool activities. It indicates that the traditional SPW treatment system could not fulfill the current water quality requirement, taking toxic DBPs into consideration. There is an urgent need to seek novel approaches and technologies to improve the conventional treatment system to finally achieve an excellent water purification purpose. Therefore, the objectives of this research are:

1) To provide a comprehensive literature review on DBPs in SPWs from several aspects, e.g., DBP regulations in different regions or by different organizations, formation mechanisms related with different disinfectants, precursors and other factors, toxicity and carcinogenicity, human exposure assessment reflected by biomarkers or epidemiological evidence, and latest approaches for the control and treatment of DBPs.

2) To investigate and evaluate the real SPW quality via a local field survey (with a reflection of low chlorine and high DBP concentrations in outdoor pools, which may threaten microbial and chemical safety of the pool participants), 
and thereafter to explore the appropriateness of using the chlorine/bromine stabilized disinfectants, i.e., TCCA, and BCDMH, for SPW disinfection.

3) To evaluate the adaptability of using RO and NF membranes to remove HAAs in simulated SPWs, and to explore the underlying rejection mechanisms by relating the performance with the physical-chemical properties of the membranes and solutes.

4) To investigate the effect of calcium ions (as a ubiquitous species in real SPWs) on HAA rejection in a more representative simulated SPW matrix by NF membranes and to explore the fundamental mechanisms that were responsible for the affected performance.

\subsection{Structure of the thesis}

Figure 1.1 illustrates the overall structure of this thesis. Chapter 1 briefly introduces the research background, problem statement, objectives and thesis structure. Chapter 2 reviews the current literature with regard to the occurrence, formation and treatment of DBPs in SPWs. This review not only shows the latest research progress on DBPs in SPWs but also provides directions and theoretical basis for future systematic investigations on DBP minimization at the source or subsequent treatment processes. Chapter 3 starts with a field survey aiming at investigating the water quality in swimming pools. This survey highlights two main issues: low disinfectant and high DBP concentrations, which may threaten both microbial and chemical safety of the swimmers. Two alternative disinfectants aiming at addressing these two issues have been attempted for SPW 
disinfection. Chapter 4 targets at using RO and NF membrane technology to remove HAAs from SPWs. Good HAA rejection under conditions of interest has been obtained and the underlying mechanism has been clearly interpreted. Chapter 5, as an extending study of Chapter 4, mainly focuses on the matrix effect on the HAA removal by NF membranes. Chapter 6 summarizes the major research findings and scientific contributions in this study and recommends the future research directions.

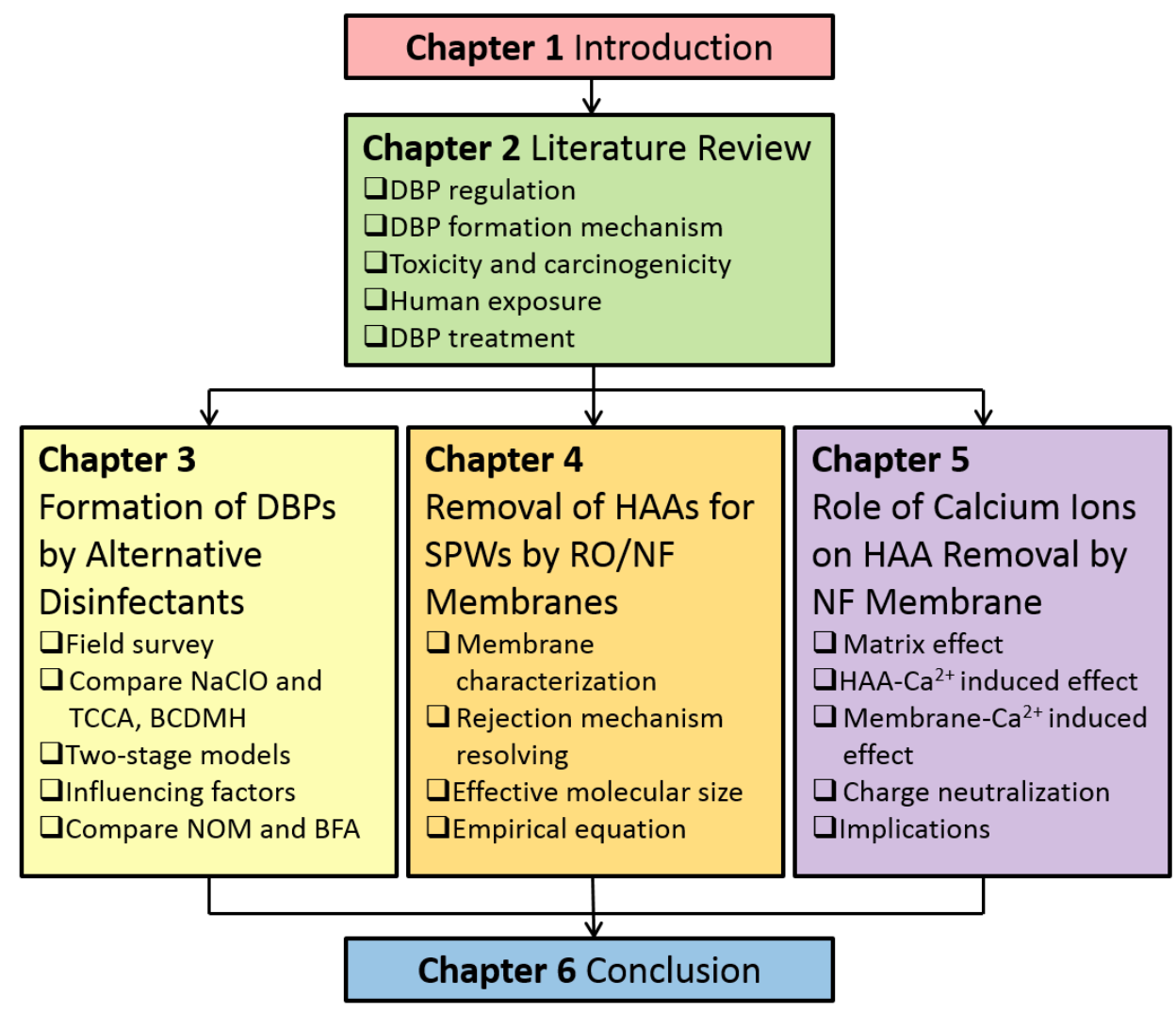

Figure 1.1 Thesis structure 


\section{Chapter 2 Literature Review}

\section{$2.1 \quad$ Introduction}

Swimming is a very popular activity for exercise and entertainment across the world. In Singapore, the government encourages every citizen to learn how to swim for fitness purpose. There are totally 26 public swimming complexes in the mini main land with an area of $\sim 700$ square kilometers (Singapore, 2014). All the universities and schools have campus pools, and every Condo provides swimming pools for its residences and many villas have their private pools. There are more than 100,000 visitors annually in each public pool based on our preliminary survey. The United States has 250,000 public pools and more than 368 million visitors every year (Zwiener et al., 2007). In Germany, there are more than 600,000 pools and the visitors reach more than 2300 per day in an outdoor pool (Glauner et al., 2005b; Zwiener et al., 2007). The numbers of pools in other European countries are also amazing, 773,000 in France, 155,000 in the UK, and 94,000 in Italy (Zwiener et al., 2007).

During pool activities, human substances (e.g., skin particles, sweat, urine, fecal, and hair) and PCPs (e.g., sunscreen, body lotion, shampoos, and other cosmetics) enter into the SPWs and become potential contaminants (see details in Section 2.3.2). For outdoor pools, the additional pollutants come from leaves and dust in the surrounding environment or from the rain water. These contaminants carry some pathogens, including bacteria, viruses and protozoa, which may result in some diseases or symptoms, e.g., gastroenteritis , dermatitis, etc. (Craun et al., 2005). Centers for Disease Control and Prevention (CDC) and EPA have 
conducted a 30-year (1971-2000) surveillance to report the waterborne outbreaks and the associated causes. During this period, > 11,000 cases of illness and $>70$ waterborne outbreaks in swimming pools were reported in the United States (Craun et al., 2005).

Disinfection plays a decisive role in killing microbial to prevent the spreading of some infectious diseases in SPWs. However, the disinfectants can react with water constituents of natural and anthropogenic origin to yield toxic DBPs (Fischer et al., 2012). The researchers became aware of the DBPs since early 1970 and more than 600 DBPs have been reported in DWs in the past 40 years (Richardson et al., 2007). THMs were the first identified DBP group with a concentration level of low to mid $\mu \mathrm{g} / \mathrm{L}$ in DWs in 1974 (Rock, 1974). Five years later, U.S. EPA regulated the THMs with a MCL of $100 \mu \mathrm{g} / \mathrm{L}$ in DWs (EPA, 1979). In 1998, the MCL of THMs was lowered to $80 \mu \mathrm{g} / \mathrm{L}$ (EPA, 1998). At the same time, HAAs, as another prevalent class of DBPs, were first time regulated with a MCL of $60 \mu \mathrm{g} / \mathrm{L}$ for the sum of chloro-, bromo-, dichloro-, dibromo-, and trichloroacetic acids. THMs and HAAs account for nearly $25 \%$ of all halogenated DBPs, which makes them of significant research values (Krasner et al., 2006).

The public attention paid to DBPs in SPWs is not as much as that in DWs according to the number of publications in the past 20 years (Figure 2.1). However, it is becoming a hot topic due to the increasing popularity of swimming activity and its associated health side-effects. In addition to NOM from the source water, body fluid and PCPs released from the swimmers make the water matrix more complicated and possess a higher potential to form regulated and emerging 
DBPs (Section 2.3.2). For example, the active ingredients, e.g., urea, in urine can react with free chlorine to form chloramine, which may lead to eye and upper respiratory tract irritation, biomarker changes in the lung, and development of asthma (Blatchley III and Cheng, 2010; Schmalz et al., 2011). The kinds and numbers of DBPs in SPWs rely on a number of other factors, for example, the type and amount of disinfectant dosed in the system, water solution properties (e.g., $\mathrm{pH}$, temperature, $\mathrm{Br}^{-}$), pool types (indoor/outdoor), or even bather activities (Section 2.3.3).

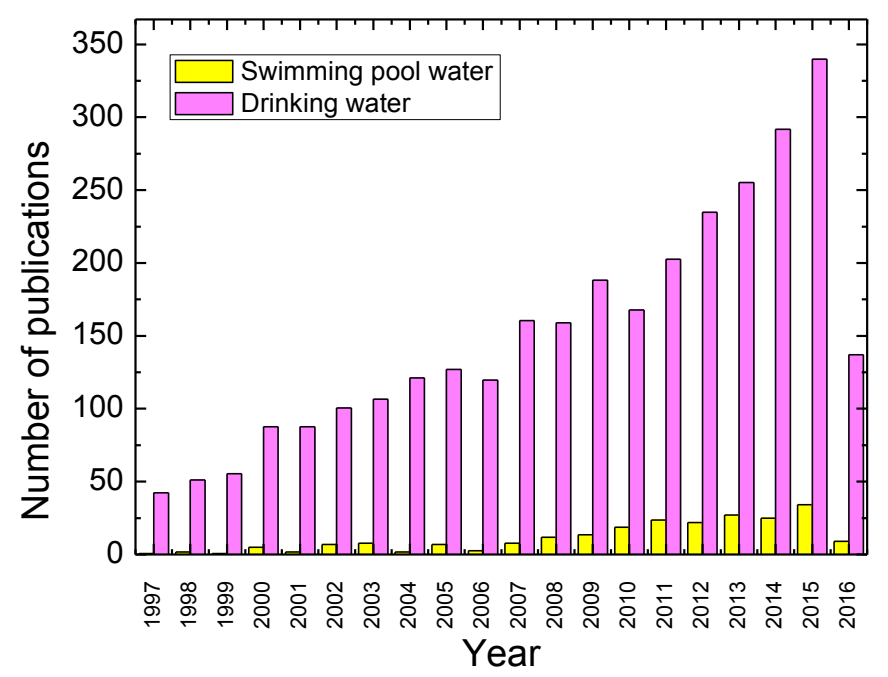

Figure 2.1 The number of publications in relation with DBPs in SPWs and DWs in the past 20 years (1997-2016). Source: Web of Science on June 22, 2016. Keywords for searching: disinfection by-product, swimming pool water/drinking water.

It is worth noting that DBP exposure during swimming was much greater than that via DW (Villanueva et al., 2006; Villanueva et al., 2007b). Only 1\% of the total uptake of THMs during pregnancy was from DW via oral ingestion, however, $23 \%$ from swimming activities by inhalation and dermal absorption (Villanueva et al., 2007b). In Section 2.5 , we reviewed how and to what extent human are exposed in DBP-contaminated swimming pool environment. Due to 
the high data availability for THMs and HAAs, we assessed the exposure by THM/HAA concentrations measured in swimmers' or workers' body matrix, including exhaled air, urine, blood, and plasma, as biomarkers. Dermal absorption and inhalation are two most important THM exposure pathways for swimmers or workers. However, HAAs, not as volatile as THMs, are exposed to swimmers mainly via ingestion ( 94\%) (Cardador and Gallego, 2011). In addition, the trace HAAs detected in workers' urine demonstrated the nonignorable role of inhalation as well. Some uncomfortable symptoms have been reported by the pool participants and its potential relationship with the pool activity has raised public attentions. Moreover, it is not accidental to observe that the DBP concentrations in SPWs are much or even several orders of magnitude higher than the MCLs in DW or SPWs, which demonstrates the traditional SPW treatment system (flocculation-sand filtration-chlorination) seems inadequate to meet the current water quality requirement. Up to date, the treatment methods specifically aimed at removing DBPs in SPWs is still in the initial stage with only laboratory-scale, or at most pilot- scale trials. Studies in the past two decades show that membrane filtration, advanced oxidation processes (AOPs), biodegradation, thermal degradation, chemical reduction and some hybrid processes are the potential DBP treatment technologies. It may provide potential directions for further research extension in these domains and will become theoretical basics for future full-scale applications.

This review overviews the latest research progress of DBPs in SPWs from the following aspects: DBP regulations in different regions or by different organizations, formation mechanisms related with different disinfectants, 
precursors and other factors, toxicity and carcinogenicity, human exposure assessment reflected by biomarkers or epidemiological evidence, and latest approaches for the control and treatment of DBPs.

\subsection{Regulations and guidelines of DBPs}

The regulations and guidelines of DBPs, mainly including THMs, HAAs, haloacetonitriles (HANs), bromated, chlorate and chlorite, in DW have been established by some global organizations or developed countries (Table 2.1). THMs were first regulated by U.S. EPA in 1979 with a MCL of $100 \mu \mathrm{g} / \mathrm{L}$ in DWs (EPA, 1979). In 1998, the MCL of THMs was lowered to $80 \mu \mathrm{g} / \mathrm{L}$ and meanwhile $\mathrm{HAA}_{5}$ (the sum of chloro-, bromo-, dichloro-, dibromo-, trichloroacetic acid) was first time regulated at $60 \mu \mathrm{g} / \mathrm{L}$ (EPA, 1998). Apart from four THM and five HAA compounds regulated by U.S. EPA, the MCL for bromate and chlorite are 10 and $1000 \mu \mathrm{g} / \mathrm{L}$ respectively. World Health Organization (WHO) has included totally 15 DBPs in its DW guideline and gives the MCL of the individual DBP (WHO, 2008). Three of them, i.e., cyanogen chloride (70 $\mu \mathrm{g} / \mathrm{L})$, n-nitrosodimethylamine $(0.1 \mu \mathrm{g} / \mathrm{l})$, and 2,4,6-trichlorophenol $(200 \mu \mathrm{g} / \mathrm{l})$, are not included in Table 2.1. Only two individual HANs, i.e., dibromoacetonitrile and dichloroacetonitrile, are regulated by WHO guideline due to the insufficient database in relation with human and animal toxicological studies for other analogs (Meike et al., 2009). DW quality standard prescribed by Singapore is wholly based on the WHO guideline, Geneva (NEA, 2008). The European Union only regulates THMs (< $100 \mu \mathrm{g} / \mathrm{L})$ and bromate $(<10 \mu \mathrm{g} / \mathrm{L})$ (Directive, 1998). 
Table 2.1 DBP regulations and guidelines in drinking water

\begin{tabular}{|c|c|c|c|c|c|c|}
\hline DBPs & $\begin{array}{l}\text { U.S. } \\
\text { EPA } \\
\text { (EPA, } \\
1998)\end{array}$ & $\begin{array}{l}\text { WHO } \\
\text { (WHO, } \\
2008 \text { ) }\end{array}$ & $\begin{array}{l}\text { Singapore } \\
\text { (NEA, } \\
\text { 2008) }\end{array}$ & $\begin{array}{l}\text { European } \\
\text { Union } \\
\text { (Directive, } \\
1998) \\
\text { MCL }^{\mathrm{a}}(\mu \mathrm{g} / \mathrm{L})\end{array}$ & $\begin{array}{l}\text { Australia } \\
\text { (Australia, } \\
\text { 2011) }\end{array}$ & $\begin{array}{l}\text { Canada } \\
\text { (Canada, } \\
2014)\end{array}$ \\
\hline $\mathrm{THMs}^{\mathrm{b}}$ & 80 & -- & -- & 100 & 250 & 100 \\
\hline Chloroform & 70 & 300 & 300 & -- & -- & -- \\
\hline Bromodichloromethane & 0 & 60 & 60 & -- & -- & -- \\
\hline Chlorodibromomethane & 60 & 100 & 100 & -- & -- & -- \\
\hline Bromoform & 0 & 100 & 100 & -- & -- & -- \\
\hline $\mathrm{HAAs}^{\mathrm{c}}$ & 60 & -- & -- & -- & -- & 80 \\
\hline Dichloroacetic acid & 0 & 50 & 50 & -- & 100 & -- \\
\hline Trichloroacetic acid & 20 & 200 & 200 & -- & 100 & -- \\
\hline Monochloroacetic acid & 70 & 20 & 20 & -- & 150 & -- \\
\hline HANs & -- & -- & -- & -- & -- & -- \\
\hline Dibromoacetonitrile & -- & 70 & 70 & -- & -- & -- \\
\hline $\begin{array}{l}\text { Dichloroacetonitrile } \\
\text { Others }\end{array}$ & -- & 20 & 20 & -- & -- & -- \\
\hline Bromate & 10 & 10 & 10 & 10 & 20 & 10 \\
\hline Chlorate & & 700 & 700 & -- & -- & 1000 \\
\hline Chlorite & 1000 & 700 & 700 & -- & 800 & 1000 \\
\hline
\end{tabular}

Note:

a MCL means maximum contaminant level.

b THMs refer to the summation of chloroform, bromodichloromethane, chlorodibromomethane, and bromoform.

${ }^{\mathrm{c}}$ HAAs refer to the summation of chloro-, bromo-, dichloro-, dibromo-, and trichloroacetic acids.

Unlike the systematic regulation of DBPs in DWs, only THMs have been regulated in some European countries in SPWs (Table 2.2). Based on DIN 19643, the MCL of THMs (calculated as chloroform) in SPWs is $20 \mu \mathrm{g} / \mathrm{L}$ in Germany (Germany, 2012). Switzerland has provided the MCL of THMs as $30 \mu \mathrm{g} / \mathrm{L}$ and Denmark has promulgated the regulation of THMs in SPWs to be under 25 and $50 \mu \mathrm{g} / \mathrm{L}$ depending on the pool types (Simard et al., 2013). The French Agency for Food, Environmental and Occupational Health \& Safety (ANSES) has set a mandatory value for THMs of $100 \mu \mathrm{g} / \mathrm{L}$ and a guide value of $20 \mu \mathrm{g} / \mathrm{L}$ (ANSES, 2012). Some other developed countries, for example, Singapore, Canada and the United States, still do not have regulations for DBPs in SPWs in spite of the high popularity of swimming activity in these regions. The MCLs of DBPs in SPWs seem comparable or even lower than these in DWs. However, the DBP concentrations in real SPWs are always much higher than those in DWs (Section 
2.3.3.1). DBPs always appear in the source or make-up water (mainly tap water) and the residual free chlorine in pools further reacts with natural and anthropogenic organic matters to product more DBPs.

Table 2.2 DBP regulations and guidelines in SPWs

\begin{tabular}{llll}
\hline Country & MCL $^{\mathrm{a}}(\mu \mathrm{g} / \mathrm{L})$ & Comments & References \\
\hline Germany & 20 & THMs calculated as chloroform & (Germany, 2012) \\
Switzerland & 30 & THMs for indoor pools & (Simard et al., 2013) \\
Denmark & 25 or 50 & THMs (depending on the type of pool) & (Simard et al., 2013) \\
Belgium & 100 & Chloroform & (Simard et al., 2013) \\
France & 100 or $20^{\mathrm{b}}$ & THMs & (ANSES, 2012) \\
United & 100 & THMs & (Simard et al., 2013) \\
Kingdom & 100 & THMs & (Simard et al., 2013) \\
Finland & 100 & & \\
\hline
\end{tabular}

Note:

${ }^{a}$ MCL means maximum contaminant level.

${ }^{\mathrm{b}}$ Mandatory value for THMs is $100 \mu \mathrm{g} / \mathrm{L}$ and the guide value for THMs is $20 \mu \mathrm{g} / \mathrm{L}$.

\subsection{DBP formation mechanism}

\subsubsection{Disinfectants and their aqueous chemistry}

\subsubsection{Different disinfectants}

Chlorination is the most commonly used disinfection approach for SPW treatment (Afifi and Blatchley Iii, 2015; Kogevinas et al., 2010; Weng and Blatchley, 2011). The chlorine based disinfectants mainly include chlorine gas $\left(\mathrm{Cl}_{2}\right)$, sodium hypochlorite $(\mathrm{NaClO})$ and calcium hypochlorite $\left(\mathrm{Ca}(\mathrm{ClO})_{2}\right)(\mathrm{WHO}$, 2006). The hydrolysis and/or dissociation of these disinfectants produce hypochlorous acid $(\mathrm{HClO})$ and hypochlorite ion $\left(\mathrm{ClO}^{-}\right)$as the active ingredients for disinfection (Eqs. (2.1)-(2.4)). The equilibrium between $\mathrm{HClO}$ and $\mathrm{ClO}^{-}$is $\mathrm{pH}$ and temperature dependent.

$$
\mathrm{Cl}_{2}+\mathrm{H}_{2} \mathrm{O} \rightarrow \mathrm{HClO}+\mathrm{HCl}
$$




$$
\begin{gathered}
\mathrm{NaClO} \rightarrow \mathrm{Na}^{+}+\mathrm{ClO}^{-} \\
\mathrm{Ca}(\mathrm{ClO})_{2} \rightarrow \mathrm{Ca}^{2+}+2 \mathrm{ClO}^{-} \\
\mathrm{ClO}^{-}+\mathrm{H}_{2} \mathrm{O} \rightarrow \mathrm{HClO}+\mathrm{OH}^{-}
\end{gathered}
$$

Bromine based disinfectants are used in pools with specific conditions, e.g., spa. Liquid bromine, following the similar hydrolysis pattern of chlorine gas, is used for the maintenance of SPWs, especially for hot tubs (Eq. (2.5)). Some European countries have practiced using the seawater as the source of SPWs since the early 1900s (Parinet et al., 2012). The bromide ion with a concentration of $\sim 70 \mathrm{mg} / \mathrm{L}$ can be oxidized by $\mathrm{HClO}$ to form $\mathrm{HBrO}$ which plays a dominant role on disinfection (Eq. (2.6)) (Borges et al., 2005; Von Gunten and Oliveras, 1998). Bromine overcomes the unstable properties of chlorine under high temperature and improves the life circle of free-halogen antimicrobial. However, bromine based disinfectants are depleted rapidly in sunlight leading to the ill-suited application in outdoor pools and spas. In addition, Parinet et al. (2012) pointed out that the formation of more toxic Br-DBPs, e.g., bromoform, dibromoacetic acid, and tribromoacetic acid, was substantial in seawater pools.

$$
\begin{aligned}
& \mathrm{Br}_{2}+\mathrm{H}_{2} \mathrm{O} \rightarrow \mathrm{HBrO}+\mathrm{HBr} \\
& \mathrm{HClO}+\mathrm{Br}^{-} \rightarrow \mathrm{HBrO}+\mathrm{Cl}^{-}
\end{aligned}
$$

Organic based disinfectants mainly include TCCA, dichloroisocyanuric acid (DCCA), and BCDMH. TCCA and DCCA are widely used in outdoor pools, especially for those exposed under strong sunlight, due to their role of chlorine 
stabilizer (Zwiener et al., 2007). The bound forms of chlorine in TCCA, i.e., monochloroisocyanurate ion $\left(\mathrm{HClCy}^{-}\right)$and dichloroisocyanurate ion $\left(\mathrm{Cl}_{2} \mathrm{Cy}^{-}\right)$, are more stable than $\mathrm{HClO}$ and $\mathrm{ClO}^{-}$under the sunlight exposure, as the former have much lower maximum absorption wavelength (Wojtowicz, 1996; 2004). Askins (2013) has pointed out that the degradation of chlorine slowed down with the presence of cyanuric acid under natural sunlight exposure. BCDMH is also sometimes used for SPW disinfection (Lourencetti et al., 2012; Richardson et al., 2010). BCDMH shows better fecal coliform killing efficiency compared to commonly used $\mathrm{NaClO}$ (Moffa et al., 2006). The hydrolysis of TCCA (similar for DCCA) and BCDMH are shown in Eqs. (2.7) and (2.8), respectively.

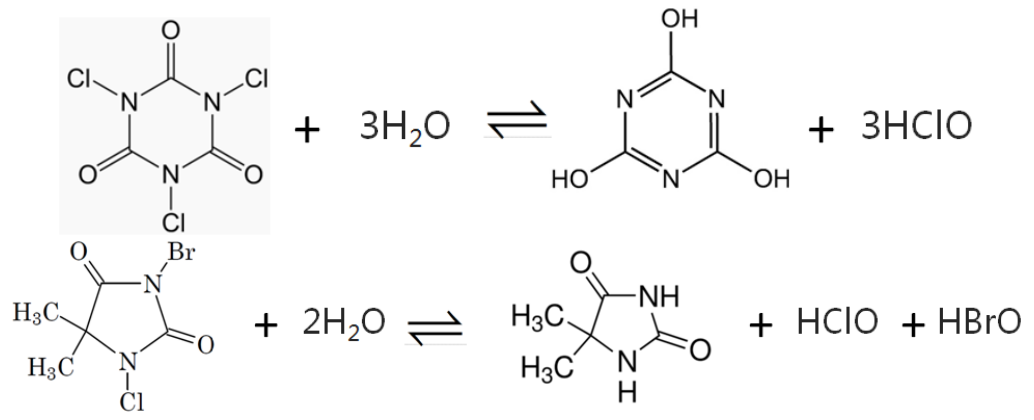

Other halogen-free disinfectants, e.g., UV and ozone, occupy the advantages to eliminating the formation of halogenated DBPs. However, they are unable to sustain long time disinfection ability due to the lack of residuals. Chlorine dioxide produces chlorite and chlorate (Eq. (2.9)), which are mandatory regulated species in DWs (Table 2.1). In order to achieve a healthier water chemistry environment, combined use of different disinfectants has raised public attention. Liviac et al. (2010) has pointed out that the combination of UV and chlorine reduced the genotoxicity compared to chlorine alone. The average total concentration of DBPs (including THMs, HAAs, HANs, and chloral hydrate) in 
SPWs disinfected by chlorine alone was significantly higher than that by combined chlorine and ozone based on an indoor pool survey (183 vs. $33 \mu \mathrm{g} / \mathrm{L}$, $\mathrm{n}=86$ ) (Lee et al., 2010). On the other hand, Richardson et al. (1999) mentioned that some emerging DBPs, e.g., 1,1-dichloropropanone and dichloroacetaldehyde, appeared at higher concentrations during the combined disinfection (ozone and chlorine) compared to chlorination only.

$$
2 \mathrm{ClO}_{2}+\mathrm{H}_{2} \mathrm{O} \rightarrow \mathrm{ClO}_{3}^{-}+\mathrm{ClO}_{2}^{-}+2 \mathrm{H}^{+}
$$

Table 2.3 Disinfectant classifications

\begin{tabular}{ll}
\hline Groups & Disinfectants \\
\hline Chlorine based disinfectants & Chlorine gas \\
& Sodium hypochlorite \\
& Calcium hypochlorite \\
Bromine based disinfectants & Bromine liquid \\
& Sodium bromide + hypochlorite \\
Organic based disinfectants & Bromochlorodimethylhydantoin \\
& (BCDMH) \\
& Trichloroisocyanutic acid (TCCA) \\
& Dichloroisocyanuric acid (DCCA) \\
& UV \\
Others & Ozone \\
& Chlorine dioxide \\
& Hydrogen peroxide/silver/copper \\
& Combined disinfectants \\
\hline
\end{tabular}

\subsubsection{Regulated disinfectant range}

Microbiologically safe SPW ensures the safety of swimmers' health. Disinfection plays a decisive role to prevent the spreading of some microbial related infectious diseases in SPWs. Active ingredients for disinfection mainly include $\mathrm{HClO}$ and $\mathrm{ClO}^{-}$, the sum of which is defined as free chlorine (White, 1999). The control of the amount of free chlorine in SPWs is essential. An excess of free chlorine may 
cause user discomfort, such as skin irritation, and insufficient free chlorine cannot ensure the effective microbial killing. Countries throughout the world have made its respective regulation for the control of free chlorine (see Table 2.4). National Environment Agency (NEA) in Singapore recommends a range of 1-3 mg/L for free chlorine (NEA, 2005). Based on Germany standard DIN19643, the free chlorine ranges from 0.3 to $0.6 \mathrm{mg} / \mathrm{L}$ for swimming pools and from 0.7 to 1.0 mg/L for hot tubs (Germany, 2012). In the U.S., National Swimming Pool Foundation (NSPF) regulates free chlorine at $1-5 \mathrm{mg} / \mathrm{L}$ and recommends an ideal range of 2-4 mg/L in real operation (NSPF, 2006). In addition, some states in the U.S. regulate their local pools individually, for example, the free chlorine ranges are $0.25-5.0,1-10$, and 1.5-5.0 mg/L for pools in Colorado, Florida, and Georgia, respectively (Colorado, 1998; Florida, 2009; Georgia, 2013). The public health department of each state is assigned for the responsibility of safe operation of local pools.

\subsubsection{DBP precursors}

DBP precursors include NOM from the filling water (surface or ground water from water treatment plants), body fluid (e.g., urine, sweat, hair, saliva, etc.) from the swimmers, and chemicals used for PCPs (e.g., cosmetics and sunscreens) released from the human skin or urine (Chowdhury et al., 2014; Zwiener et al., 2007). Different from DW, where NOM is the main organic source, the addition of body fluid and PCP chemicals makes the SPW matrix more complicated and contributes to the potential formation of more emerging DBPs with unknown toxicity. There have been considerable studies reporting DBP formation in the 
presence of these substances under real or simulated swimming pool conditions (Kanan and Karanfil, 2011; Kim et al., 2002).

\subsubsection{Natural organic matter}

Similar to DW, NOM coming from the source water (mainly tap water) does exist in SPW matrix. Humic substances (i.e., humic and fulvic acids) are the major fractions, which account for 52-70\%. A large portion of NOM $(\sim 55-80 \%)$ has a molecular weight less than $1 \mathrm{kDa}$ (Fan et al., 2001). Apart from humic fractions, NOM contains more other hydrophilic substances, e.g., transphilic acids, proteins, amino acids and carbohydrates, which account for 20-40\% (Fan et al., 2001). NOM composition can also be categorised by some fractionation techniques as humic acids and hydrophobic/hydrophilic acid/base/neutral compounds (Matilainen and Sillanpää, 2010). NOM is commonly quantified as total organic carbon (TOC) or dissolved organic carbon (DOC) due to its complicated composition. It has been reported that NOM concentrations in DWs are 0.7-6.7 mg TOC/L in the U.S. (Kanan and Karanfil, 2011), and 2.6-4.7 and 1.8-5.4 mg DOC/L in Norway and Australia (Fabris et al., 2008), and 1.1-1.4 mg TOC/L in the local context (Table 3.4). The total nitrogen (TN) in the local context is 2.15 $\mathrm{mg} / \mathrm{L}$ (nitrate-nitrogen accounts for $92.1 \%$ ) is much higher than that in the U.S. (0.1-0.3 mg/L). NOM has been proven to possess a high reactivity toward the formation of DBPs (e.g., THMs, HAAs, HANs, and HNMs) during chlorination (Kanan and Karanfil, 2011; WHO, 2008). The reduced TOC in treated SPWs or raw water corresponding to the substantially reduced the formation potential of adsorbable organic halogens and THMs (AOX-FP, THM-FP), confirms that NOM is an effective precursor for DBP formation (Fabris et al., 2008; Glauner 
et al., 2005a; Glauner et al., 2005b). Fabris et al. (2008) have reported a numerical relation between NOM and THM-FP as 10-40 $\mu \mathrm{g}$ THM-FP/mg DOC. Therefore, the efficient removal of NOM in the SPW treatment system could be an immediate and effective method to reduce the DBP formation from the source.

\subsubsection{Body fluid}

Human body fluids, including urine, sweat, hair, saliva, etc., are released from swimmers during pool activities, among which urine and sweat are two main groups of contaminants (Lamont Bradford, 2014). Judd and Black (2000) have pointed out the actual pool condition is roughly equal to $200 \mathrm{ml}$ sweat and $50 \mathrm{ml}$ urine per cubic meter of pool water. The release of urine and sweat into swimming pools has been estimated in a range of 25-77.5 and 200-1000 mL/bather, respectively (De Laat et al., 2011; WHO, 2006). Similar levels of urine and sweat release from swimmers have been reported in other literature studies, i.e., 54.7-117 and 823-1760 mL/bather by Weng and Blatchley (2011), and 20-80 and 100-1000 mL/bather by Florentin et al. (2011). Compared to urine, sweat release is much higher especially during intensive exercises in pools with high temperatures. The sweat release rate is $0.04-0.91 \mathrm{~L} / \mathrm{m}^{2} / \mathrm{h}$ as summarized by Keuten from previous publications and $0.1-0.8 \mathrm{~L} / \mathrm{m}^{2} / \mathrm{h}$ as obtained from his own laboratory and on-site experiments (Keuten et al., 2014).

Urea is the main component in urine and sweat, with a mean concentration of 10240 and $680 \mathrm{mg} / \mathrm{L}$, which accounts for $84 \%$ and $68 \%$ of $\mathrm{TN}$, respectively (WHO, 2006). Urea concentrations in SPWs have been reported in many literature studies, e.g., 0.01-0.11 mg/L by Afifi and Blatchley Iii (2015), 0.50- 
$2.12 \mathrm{mg} / \mathrm{L}$ by Schmalz et al. (2011), 0.12-3.6 mg/L by De Laat et al. (2011), 0.01$4.02 \mathrm{mg} / \mathrm{L}$ by Blatchley III and Cheng (2010). Our preliminary survey showed the urea in Singapore pools were $0.05-0.82 \mathrm{mg} / \mathrm{L}$. The urea concentration of an indoor pool increased by $72.4-155 \mu \mathrm{g} / \mathrm{L}$ daily during a national swimming competition, which was equivalent to $0.56-1.20 \mathrm{~g}$ urea or $0.26-0.56 \mathrm{~g}$ TN release by each bather per day (Weng and Blatchley, 2011). Similar urea release of 0.8$1.5 \mathrm{~g} / \mathrm{b}$ ather has been reported (WHO, 2006). The average release of pollutants during 30 min exercise is $250,77.3,37.1$ and $10.1 \mathrm{mg} /$ bather for non-purgeable organic carbon, TN, urea and ammonium, respectively (Keuten et al., 2014). Intensive usage of pools results in the substantial introduction of pollutants (e.g., urea, TOC, and TN), leading to adverse effects on water quality (Afifi and Blatchley Iii, 2015; De Laat et al., 2011; Weng and Blatchley, 2011).

Apart from urea, creatinine (670-2150 mg/L), hippuric acid (50-1670 mg/L), citric acid (90-930 mg/L), ammonia (200-730 mg/L), uric acid (40-670 mg/L), glycine $(90-450 \mathrm{mg} / \mathrm{L})$, histidine $(40-330 \mathrm{mg} / \mathrm{L})$ present with substantial concentrations in urine act as DBP precursors in SPWs as well (Beach, 1971). A large portion of these nitrogenous compounds (inclusive of urea) containing various functional groups $\left(-\mathrm{NH}_{2},-\mathrm{COOH}\right)$ and forms of bound nitrogen have been proven as efficient trichloramine precursors, particularly at acidic and neutral pH conditions (Afifi and Blatchley Iii, 2015; Blatchley III and Cheng, 2010; De Laat et al., 2011; Lian et al., 2014; Schmalz et al., 2011).The release of nitrogenous compounds into swimming pools is between $320-840$, 30-60, 15-50, 10-25 and 400-1000 mg/bather for urea, ammonia, amino-acids, creatinine and TN, respectively (Florentin et al., 2011). 
In the lab-scale experiments, different recipes for body fluid analog (BFA) have been proposed to mimic swimmers' body excretions. Urea (14800 mg/L), creatinine (1800 mg/L), hippuric acid (1710 mg/L), histidine (1210 mg/L), citric acid (640 mg/L), uric acid (490 mg/L), ammonium chloride (2000 mg/L), and sodium phosphate $(4300 \mathrm{mg} / \mathrm{L})$ are a BFA recipe widely used for DBP formation potential tests in simulated pool conditions (Judd and Bullock, 2003; Kanan and Karanfil, 2011). This BFA represents a mixture of urine and sweat with a volume ratio of $1: 4$, in a concentration of $\sim 5700 \mathrm{mg}$ TOC/L and $~ 8700 \mathrm{mg}$ TN/L. In the early stage, Judd and Black (2000) used a far more complicated BFA recipe with some additional inorganic compounds. This recipe is more representative to the real urine or sweat compositions (Beach, 1971), but the additional portion does not essentially affect the DBP formation. Kanan and Karanfil (2011) used three BFAs to study their difference in DBP speciation and found some comparable and dramatically different DBP formation results due to their variable compositions. DBP FP tests of individual component showed that citric acid made significantly greater contribution to THM and HAA formation compared to other BFA species (Kanan and Karanfil, 2011), and that acid amides, particularly urea, ammonium ions and a-amino acids were effective trichloramine precursors (Schmalz et al., 2011).

The high potential of DBP formation by human fluid raises the public awareness of the importance of hygiene behaviour, i.e., have a shower before entering into the pools, do not urinate during swimming. However, a public survey among 1000 adults in the U.S. in 2009 showed that $35 \%$ of pool participants skipped the 
shower before swimming and $17 \%$ had urination experience (Wiant, 2011). It also showed that $84 \%$ of the Americans believed their fellow swimmers engaged in unhygienic behaviours at public pools. Keuten et al. (2014) have found that, at high exercise levels $63 \%$ of pollutants in pools come from unhygienic behaviour, of which $31 \%$ is a result of none pre-swim shower and $32 \%$ results from urination. The avoidance of unhygienic behaviour could potentially reduce the DBP formation by $55 \%$ in $35{ }^{\circ} \mathrm{C}$ and up to $68 \%$ in $25{ }^{\circ} \mathrm{C}$, and this percentage is expected to increase at lower exercise levels (Keuten et al., 2014). A pre-swim shower as short as $60 \mathrm{~s}$ removed a significant amount of pollutants from human bodies (Keuten et al., 2012). Therefore, it is essential to encourage or even enforce the swimmers to develop good hygiene habits.

\subsubsection{Personal care products}

In addition to the commonly discussed NOM and BFA, PCPs (e.g., sunscreen, body lotion, hand soaps, laundry detergents, shampoos, hair gels) released by bathers in SPWs could be the potential precursors of chlorinated and oxidized or nitrogenous DBPs (Balmer et al., 2005). The more detectable PCPs in SPWs than in DWs is mainly attributed to the lower dilution ratio and high recirculation of SPWs. For example, the concentration of UV filters reaches up to $\mu \mathrm{g} / \mathrm{L}$ in SPWs or shower wastewater, which is several orders of magnitude higher than that in surface water (ng/L) (Bottoni et al., 2014; Lambropoulou et al., 2002).

The sunscreen is widely used during the summer period when pool activities are most popular. It aims at preventing skin damage under solar radiation via some commonly used organic reagents, e.g., 4-Methylbenzylidine camphor (Serpone 
et al., 2002). Zwiener et al. (2007) have identified 5 active ingredients of sunscreens, namely 2-hydroxy-4-methoxybenzophenone, ethylhexyl methoxycinnamate, 2-ethylhexyl-2-cyano-3,3-diphenyl-2-propenoate, 2-phenyl1H-benzimidazole-5-sulfonic acid, and 4-methylbenzylidene camphor, with the sum concentration ranging from single-digital to dozens of $\mu \mathrm{g} / \mathrm{L}$ in outdoor pools. The higher bather load in the baby pool explained its higher sunscreen concentration than that in the adult pool. Among these sunscreen ingredients, 2hydroxy-4-methoxybenzophenone produced considerably TOX (total organic halides) and THM after chlorination (Zwiener et al., 2007). The solar irradiation of a sunscreen agent, i.e., octyl-dimethyl-p-aminobenzoic acid, demonstrated its photolysis mainly via dealkylation and hydroxylation. In addition to the phototransformation products of octyl-dimethyl-p-aminobenzoic acid detected in seawaters, the identification of some chlorinated intermediates in chlorinated SPWs demonstrates its potential role in DBP formation (Sakkas et al., 2003). Vidal et al. (2010) developed a method to identify six active ingredients commonly used in sunscreens (e.g., isoamyl 4-methoxycinnamate, 3-(4'methylbenzylidene)camphor, 2-hydroxy-4-methoxybenzophenone, 2-ethylhexyl 2- cyano-3,3-diphenylacrylate, 2-ethylhexyl 4-dimethylaminobenzoate, and 2ethylhexyl 4-methoxycinnamate). Only the former two species were detectable in a public swimming pool with concentrations of $\sim 0.7 \mu \mathrm{g} / \mathrm{L}$ and $<$ LOQ (limit of quantification), respectively. However, they were not detectable in a private pool, possibly due to the low bather load (Vidal et al., 2010).

The studies reported the presence of sunscreen constitutes in SPWs is limited due to the high detection limit of current used analytical methods and lack of public 
concerns. Researchers started to conduct the laboratory experiments to investigate their roles in DBP formation in the late 2000s. The halogenation of sunscreen regents relies on many parameters, e.g., chlorine concentration, solution $\mathrm{pH}$, the presence of bromide ions, the compound structure, etc. (Nakajima et al., 2009; Negreira et al., 2008). Wang et al. (2013) conducted the DBP FP tests for 4 body lotions and 4 sunscreens, and found the formation of an emerging DBP group, i.e., halobenzoquinones, such as 2,6-dichloro-1,4benzoquinone with concentrations up to $>5000 \mathrm{ng} / \mathrm{L}$ and 2,6-dichloro-3-methyl$(1,4)$ benzoquinone with a concentration range of $<0.1-4.0 \mathrm{ng} / \mathrm{L}$. Two orders higher of 2,6-dichloro-1,4-benzoquinone or other more detectable halobenzoquinone (e.g., 2,3,6-trichloro-(1,4)benzoquinone, 2,3-dibromo-5,6dimethyl-(1,4)-benzoquinon, and 2,6-dibromo-(1,4)benzoquinone) in SPWs than in tap water was attributed to the higher presence of these sunscreen precursors in SPWs (Wang et al., 2013). Nakajima et al. (2009) tested the chlorination of two sunscreen agents, i.e., octyl dimethyl- $p$-aminobenzoate and octyl-pmethoxycinnamate, in model swimming pool conditions and found the intensive appearance of some chlorine-substituted compounds. The mutagenicity of the chlorinated octyl-p-methoxycinnamate increased rapidly during the initial reaction stage and later reduced to the control level, indicating the formation of some unstable mutagenic intermediates. Negreira et al. (2008) tested the stability of 2-ethylhexyl 4-(dimethylamino) benzoate, 2-ethylhexyl salicylate, and 2hydroxy-4-methoxybenzophenone, and identified their possible halogenated byproducts under chlorine exposure. The formation of DBPs was observed via aromatic substitution of hydrogen by halogen or cleavage of the carbonyl bond. 
Weng et al. (2014) found N,Ndiethyl- m-toluamide, caffeine, and tri(2chloroethyl)- phosphate were the only 3 of 32 pharmaceuticals and personal care products (PPCPs) present in SPWs at measurable concentrations. The absence of other PPCPs may be attributed to their chlorine-susceptible properties (transfer to DBPs in the presence of chlorine). Conversely, the accumulation of some PPCPs in SPWs may be caused by the slow reaction rate with chlorine, slow transfer from liquid to gas phases, or inefficient metabolism in humans (Weng et al., 2014). Parabens (known as hydroxybenzoate esters) are also widely used as preservatives in PCPs due to their broad antimicrobial spectrum and solubility in water (Alcudia-León et al., 2013; Terasaki and Makino, 2008). Terasaki and Makino (2008) first time showed the appearance of parabens and its corresponding Cl-DBPs in SPWs. They found that benzylparaben as one of the parabens was detected in SPWs at concentrations up to $28 \mathrm{ng} / \mathrm{L}$ (Terasaki and Makino, 2008). They also reported that a dichlorinated isopropylparaben was present in SPWs with a maximum concentration of $25 \mathrm{ng} / \mathrm{L}$, and a dichlorinated methylparaben and a monochlorinated benzylparaben were detectable but lower than LOQs. Triclosan as an antibacterial agent, widely present in hand soaps, toothpastes, deodorants, mouthwash, etc., is a chlorine-sensitive compound. The formation of chloroform and other Cl-DBPs has been reported when it is under chlorination or chloramination (Greyshock and Vikesland, 2006; Rule et al., 2005). 


\subsubsection{DBPs under various conditions}

\subsubsection{DBPs in SPWs and DWs}

Richardson et al. (2007) have summarized that a huge number of DBPs (> 600) have been detected in DWs by 2007. However, a so-far comprehensive field survey conducted also by Richardson in Spain in 2010 showed more than 100 DBPs in two large public swimming pools disinfected with $\mathrm{NaClO}$ and $\mathrm{BCDMH}$, respectively. These DBPs included THMs, HAAs, HNMs, haloacids, halodiacids, haloaldehydes, halonitriles, haloketones, haloamides, haloalcohols, and other halogenated and non-halogenated DBPs, many of which have yet been reported in SPWs and are even not exist in the current mass spectral library database (Richardson et al., 2010). Zwiener et al. (2007) has used GCMS to identify 20 DBPs, including HAAs, other haloacids (e.g., 2,2-dichloropropanoic acid, 2chloro-2-methylpropanoic acid, etc.), haloketones (e.g., 1,3-dichloropropanone, etc.), and dichloromethyl methyl sulfone in two private outdoor swimming pools treated with stabilized chlorine in the U.S. In swimming pool treatment system, chlorination is the most widely applied or only designated disinfection approach. For example, France only permits the use of chlorine-based disinfectants (e.g., sodium hypochlorite, calcium hypochlorite or stabilized chlorine) in public pools since 2011 (Parinet et al., 2012). In Singapore, NEA authorizes chlorination as the sole disinfection method in SPW treatment. However, other potential alternatives, e.g., ozone, chloramines, chlorine dioxide, commonly applied in DWs originally aiming at reducing Cl-DBP formation, may produce far more kinds of emerging non-chlorinated DBPs (Richardson et al., 2007; Sadiq and Rodriguez, 2004). 
A large population-based survey $(n=500)$ conducted in the U.S. reported the average THM and HAA concentrations of 38 and $23 \mu \mathrm{g} / \mathrm{L}$ respectively in DWs (Richardson et al., 2007). However, DBPs (HAAs in particular) in U.S. pools are far more than these values (see Table 2.5 and Table 2.6). The higher DBP concentrations in SPWs than in DWs could be attributed to the following reasons:

1) The concentration of organic matters in SPWs (high-ppm levels, single- or even two-digit) is higher than that in DWs (low-ppm levels, single-digit or even less) (Kanan and Karanfil, 2011; Kanan et al., 2015; Wang et al., 2014); 2) There are more organic species in SPWs (body fluid and PCPs in addition to NOM, see Section 2.3.2); 3) The recirculation of SPW system results in DBP accumulation. A study shows only about $1 \%$ of fresh water is added to the pools to offset the daily water consumption via evaporation and other mechanisms, and therefore the retention time of SPWs reaches as high as 100 days (Arnaud, 2016).

\subsubsection{DBPs in different countries}

DBPs in pools in different countries show different patterns. We used THM and HAA as a screen of other potential DBPs due to their high availability. The THM concentrations reported in current studies are mostly in two-digital $\mu \mathrm{g} / \mathrm{L}$ levels or occasionally slightly higher than $100 \mu \mathrm{g} / \mathrm{L}$ with one exception, i.e., $320 \mu \mathrm{g} / \mathrm{L}$ in a French study (see Table 2.5). The high concentration of THMs $\left(\mathrm{CHBr}_{3}\right.$ accounts for $~ 94 \%$ ) in this study is attributed to the use of bromide-rich seawater (68.1-106.7 $\mathrm{mg} / \mathrm{L}$ as $\mathrm{Br}^{-}$) as the pool filling water (Parinet et al., 2012) (see details in Section 2.3.3.4). In addition, $\mathrm{CHBr}_{3}$ is much easier to be accumulated with 
time than $\mathrm{CHCl}_{3}$ (as the main THM species for chlorination, vapour pressure: 5.4 vs. $196 \mathrm{mmHg})$.

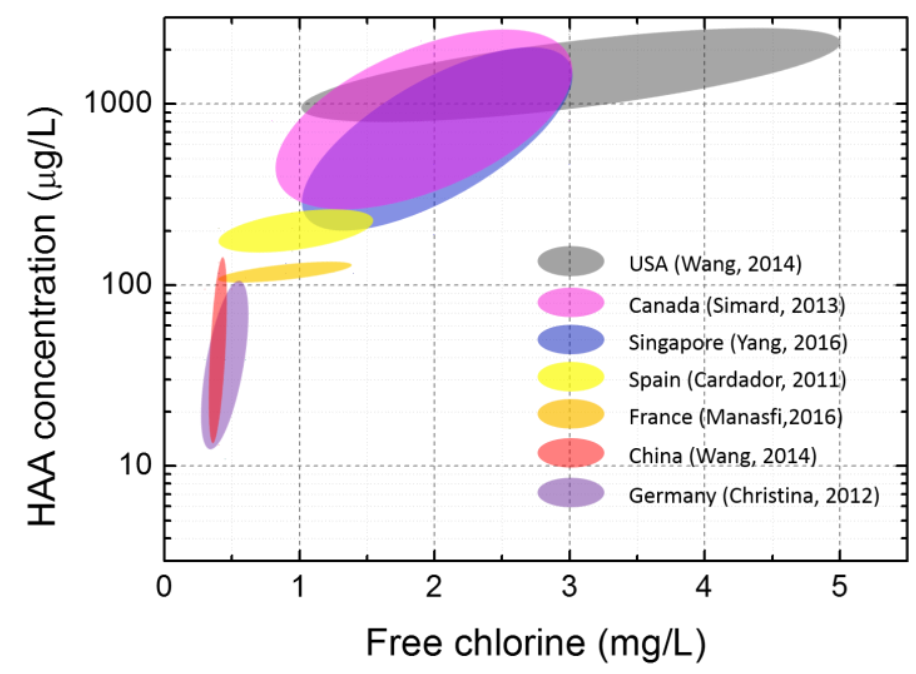

Figure 2.2 The relationship between the regulated range of free chlorine and the HAA concentrations in SPWs in different countries. The coordinates of the ellipse vertexes representing the regulated chlorine ranges and reported HAA concentrations.

The HAA concentrations in SPWs differ significantly in different countries. For example, HAAs were in average only $39 \mu \mathrm{g} / \mathrm{L}$ in a German pool (Schmalz, 2012) and 95 or $156 \mu \mathrm{g} / \mathrm{L}$ in China pools (Wang et al., 2014). Nevertheless, several studies have reported the average HAA concentrations with more than $1000 \mu \mathrm{g} / \mathrm{L}$ in the U.S. pools (Table 2.6). In addition, moderate HAA concentrations were reported for Spain and France pools. The significant gaps between these values attribute in a large extent to the different ranges of disinfectant concentrations in pools regulated by different countries, for example, only $0.3-0.6 \mathrm{mg} / \mathrm{L}$ for German pools and 0.3-0.5 mg/L for China pools, but 1-5 mg/L for U.S. pools by NSPF and 1-3 mg/L for Singapore pools (Table 2.4). Figure 2.2 illustrates that HAA concentrations increase significantly with increasing chlorine in some pool- 
popularity countries. Other potential factors, e.g., the frequency of pool usage, the hygiene habits of the bathers, may contribute to the discrepancies of DBP concentrations as well.

\subsubsection{DBPs formed by different disinfectants}

Chlorination is the most commonly used disinfection technique for SPW treatment. Chloroform, di- and trichloroacetic acids are the main THM and HAA species for chlorinated pools, and bromoform, di- and tribromoacetic acids are for brominated pools or chlorinated pools with bromide residuals (Table 2.5 and Table 2.6). For example, bromoform accounts for only $14 \%$ for chlorinated pools with minor $\mathrm{Br}^{-}$in source water and the proportion increases up to $95 \%$ during bromination by BCDMH (Lourencetti et al., 2012; Richardson et al., 2010). Chlorinated isocyanurates (e.g., TCCA and DCCA) as stabilized forms of chlorine are easy for handling (manual input) and resistant to UV irradiation, therefore are commonly used in private or outdoor pools exposed under sunlight (Bernard et al., 2008; Zwiener et al., 2007). A field study showed that DCCA reduced the DBP formation up to one order of magnitude compared to common bleach (e.g., sodium hypochlorite, calcium hypochlorite), which may be attributed to the more stable bound-chlorine species, i.e., $\mathrm{HClCy}^{-}$and $\mathrm{Cl}^{2} \mathrm{Cy}^{-}$, than $\mathrm{HClO}$ and $\mathrm{ClO}^{-}$, although other parameters may contribute as well (Parinet et al., 2012; Wojtowicz, 2004).

Alternative disinfection methods other than chlorination or bromination are also used for SPWs in some regions. In Korea, chlorine alone, the combined use of ozone and chlorine (ozone/chlorine), and a technique that uses electrochemically 
generated mixed oxidants (EGMOs), are three most commonly used disinfection methods. Ozone/chlorine seems the most chemical-safe method in terms of the formation of THMs, HAAs, HANs and chloral hydrate (Lee et al., 2009; Lee et al., 2010). The geometric mean concentrations of THMs in indoor SPWs were $32.9 \pm 2.4,23.3 \pm 2.2$, and $58.2 \pm 1.7 \mu \mathrm{g} / \mathrm{L}$ for chlorine $(\mathrm{n}=72)$, ozone/chlorine $(\mathrm{n}=$ 86) and EGMO ( $\mathrm{n}=25)$, respectively (Lee et al., 2009). Ozone as a partial substitute of chlorine reduced the total chorine dosage therefore reduced the formation of Cl-DBPs. Nevertheless, ozone promoted the formation of some extra halogen-free DBPs, e.g., bromate and nitrate, which are also toxic substances (Lee et al., 2010; Michalski and Mathews, 2007). A study in Poland showed that SPWs treated with chlorine dioxide had higher chlorite and chlorate compared to these with ozone (Michalski and Mathews, 2007). These halogenfree DBPs had also been found in DWs treated with ozone or chlorine dioxide (Sadiq and Rodriguez, 2004).The combined use of chlorine and UV had advantages of reducing genotoxicity compared to chlorination alone, which might be due to the less formation of Cl-DBPs (Liviac et al., 2010). However, UV irradiation promotes the formation of some nitrogenous DBPs (e.g., $\mathrm{CH}_{3} \mathrm{NCl}_{2}$, $\mathrm{CNCHCl}_{2}$, and $\mathrm{CNCl}$ ) which are found more toxic than the carbon-based DBPs (Muellner et al., 2007a; Weng et al., 2012). Cassan et al. (2006) found the medium-pressure UV irradiation increased chloroform and bromodichloromethane via increased active chlorine or a progressive transformation of $\mathrm{CHBr}_{3}$. Chloramination reduced the overall levels of regulated DBPs (e.g., THMs and HAAs) compared to chlorination but produced other unregulated DBPs, e.g., cyanogen chloride $(\mathrm{CNCl})$ and n-nitrosodimethylamine (Richardson et al., 2007; WHO, 2008). 
2.3.3.4 Role of water solution properties

In addition to the types and nature of disinfectants or organisms (see Section 2.3.1 and 2.3.2) in SPWs, the water solution properties, including $\mathrm{pH}$, temperature, the presence of bromide, etc., play significant roles in DBP formation as well.

Proper $\mathrm{pH}$ adjustment is an important process to ensure the disinfection efficiency. In addition, an appropriate $\mathrm{pH}$ range secures user comfort and avoids pipe corrosion. Different ranges of $\mathrm{pH}$ have been regulated in different countries and for different types of pools or disinfectants (see Table 2.4). The $\mathrm{pH}$ range is 7.2-7.8 for pools in the U.S., Canada, Australia and Singapore, however, wider ranges for pools in Germany (6.5-7.6), Spain (6.8-8.0), China (6.8-8.5 or 6.0-9.0). In general, lower $\mathrm{pH}$ is favoured as $\mathrm{HClO}$ is a more effective disinfection species than $\mathrm{ClO}^{-}$(Sadiq and Rodriguez, 2004). In terms of the effect of $\mathrm{pH}$ on DBP formation, Hansen's group has made some systematic investigations (Hansen et al., 2013; Hansen et al., 2012a; Hansen et al., 2012b). In general, with the decreasing $\mathrm{pH}$ within the range of 6.0-8.0, THM formation decreased gradually, but $\mathrm{HAN}$ and $\mathrm{NCl}_{3}$ formation increased, and $\mathrm{HAA}$ formation remained constant or decreased. The formation of THMs contains the alternate steps of hydrolysis and halogenation, which are favoured under high $\mathrm{pH}$ conditions (Bougeard et al., 2008). HANs as one group of THM precursors are expected to be increased due to their reduced hydrolysis under low pH (Teo et al., 2015). The conflicting results for HAAs may thank to the difference of organic sources in these two experiments, one is from particles of swimming pool filters and the other is from BFA (Hansen et al., 2012a; Hansen et al., 2012b). Acidic and neutral pHs are the 
most favourable conditions for $\mathrm{NCl}_{3}$ formation. Urea as one of the most effective $\mathrm{NCl}_{3}$ precursors has a relative $\mathrm{NCl}_{3}$ formation of $96 \%$ at $\mathrm{pH} 2.5$ however only $24 \%$ at $\mathrm{pH} 7.7$ (Schmalz et al., 2011). Low $\mathrm{pH}$ promotes the formation of $\mathrm{HClO}$ and therefore results in the protonation of bound nitrogen. In addition, low $\mathrm{pH}$ slows down $\mathrm{NCl}_{3}$ decay (Schmalz et al., 2011). Similarly, Kanan (2010) found three groups of DBPs (i.e., THMs, HAAs, and HNMs) decreased with decreasing $\mathrm{pH}$. Specifically, the formation of THMs and HAAs decreased by 40-60\% when pH reduced from 8 to 6 . Hansen et al. (2013) recommended a $\mathrm{pH}$ range of 6.87.5 for SPWs based on the calculated genotoxicity of several DBP groups (including THMs, HANs and $\mathrm{NCl}_{3}$ ). Nevertheless, a comprehensive toxicity assessment to a wider range of DBPs existed in SPWs is necessary to obtain a more reliable $\mathrm{pH}$ range.

The temperature of SPWs also influences the DBP formation. Simard et al. (2013) found higher concentrations of THMs, HAAs and inorganic chloramine in heated than non-heated outdoor pools ( 23 vs. $\left.26^{\circ} \mathrm{C}\right)$. An indoor swimming pool survey $(n=10)$ in Canada illustrated higher halobenzoquinone formation at higher temperatures (Wang et al. 2013). Kanan and Karanfil (2011) found higher temperature contributed to higher THM than HAA yields, which is possibly due to thermal decarboxylation of HAAs to the corresponding THMs (Lifongo et al., 2010). The DBP formation affected by temperature could be a combined result of several contributing factors, e.g., $\mathrm{HClO}$ ionization, the kinetics of reactions between chlorine and organic matters, heat-promoted hydrolysis of bound chlorine/bromine if stabilized disinfectants are used, thermal stability of DBPs, etc. In addition, the sweat release rate increased from $0.1-0.2$ to $0.8 \mathrm{~L} / \mathrm{m}^{2} / \mathrm{h}$ with 
the temperature increase from 29 to $35{ }^{\circ} \mathrm{C}$, which indicates temperature to be a main parameter to restrain the continual anthropogenic pollutant release from the swimmers (Keuten et al., 2014).

The bromide present in the source water or as impurities in chemicals for SPW treatment plays a noticeable role in the formation of Br-DBPs. The SPWs are mainly supplied by DWs with a Br- range of 36-2230 $\mu \mathrm{g} / \mathrm{L}$ based on a survey during 1997-1998 by U.S. EPA or with a small portion supplied by seawater with 70-90 mg/L Br- (Kanan, 2010; Manasfi et al., 2016; Parinet et al., 2012). Bromoform, dibromoacetic acid, tribromoacetic acid, dibromoacetonitrile, tribromonitromethane and bromonitromethane are the predominant species of THMs, HAAs, HANs and HNMs in pools with the presence of bromide (Kanan, 2010; Manasfi et al., 2016; Parinet et al., 2012). The THM and HAA concentrations reached as high as 996 and $2233 \mu \mathrm{g} / \mathrm{L}$ respectively (bromoform accounted for $93 \%$ of THMs, and dibromoacetic acid, tribromoacetic acid accounted for $70 \%$ of HAAs) in one of the eight chlorinated seawater pools, which are far more than the average in chlorinated freshwater pools as shown in Table 2.5 and Table 2.6 (Parinet et al., 2012). However, except THMs with comparable values, the concentrations of HANs, haloketones, HAAs, THAs and HNMs in chlorinated freshwater pools were much higher than those in chlorinated seawater pools (Manasfi et al., 2016). This discrepancy may be related to other factors e.g., the number of swimmers, solution $\mathrm{pH}$ and temperature, location of pools, physicochemical properties of DBPs, etc. Nevertheless, the cytotoxicity and genotoxicity study based on 18 DBPs (including 6 HAAs, 6 haloacetamides, 3 HANs and 3 THMs) showed that Br- 
DBPs were substantially more toxic than their chlorinated analogs (Plewa et al., 2008). Hence, the use of freshwater with high bromide or seawater should be avoided to reduce the formation of more toxic Br-DBPs.

\subsubsection{Role of pool types}

The coverage of the pools, e.g., indoor or outdoor, has an impact on certain DBP formation. THM and HAA concentrations in outdoor pools were found to be higher than those in indoor pools based on two studies (Cardador and Gallego, 2011; Simard et al., 2013). The entries of addition precursors from the external environment, e.g., rains, dust, leaves, for outdoor pools might be an appropriate interpretation. The doubled TOC concentration in outdoor pools than indoor pools effectively validated the above assumption (Simard et al., 2013). In addition, prolonged exposure of outdoor pools under solar radiation could contribute to higher THM and HAA yields as well (Liu et al., 2006). However, the lower inorganic chloramine concentration in outdoor pools was also possibly caused by its photodegradation by UV rays (Simard et al., 2013). On the other hand, indoor environment may accumulate the volatile DBPs in the gaseous phases above the pool water levels if the ventilation conditions are insufficient (Catto et al., 2012). Zwiener et al. (2007) pointed out that swimmers and even non-swimmers in indoor pools are exposed to a larger extent than in outdoor pools, via inhalation of volatile DBPs (e.g., THMs and $\mathrm{NCl}_{3}$ ). Similarly, higher DCAA and TCAA were detected in workers' urine samples in indoor pools than outdoor pools although outdoor pools generally had higher HAAs (Cardador and Gallego, 2011). However, Li and Blatchley (2007) and Wang et al. (2014) found both indoor and outdoor pools in the U.S. had similar THM and HAA 
concentrations. Therefore, final DBP concentrations in pools rely on the combined effects of formation, transformation and degradation. 
Table 2.4 Regulated conditions for the operation of swimming pools

\begin{tabular}{|c|c|c|c|c|}
\hline $\begin{array}{l}\text { Countries } \\
\text { US }\end{array}$ & Free chlorine & Combined chlorine $\left(\mathrm{MCL}^{\mathrm{a}}\right)$ & $\mathrm{pH}$ & Reference \\
\hline \multirow[t]{2}{*}{ US } & 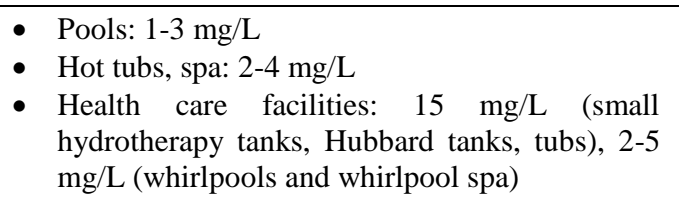 & & - $7.2-7.8$ & (Bottoni et al., 2014) \\
\hline & $\begin{array}{l}\text { - } 1-5 \mathrm{mg} / \mathrm{L} \text { (an ideal range of } 2-4 \mathrm{mg} / \mathrm{L} \text { in real } \\
\text { operation) }\end{array}$ & $\begin{array}{l}\text { - Pools: } 0.2 \mathrm{mg} / \mathrm{L} \\
\text { - Spa: } 0.5 \mathrm{mg} / \mathrm{L}\end{array}$ & - $7.4-7.6$ & (NSPF, 2006) \\
\hline $\begin{array}{l}\text { Canada } \\
\text { (Quebec) }\end{array}$ & $\begin{array}{l}\text { - Indoor pools: } 0.8-2 \mathrm{mg} / \mathrm{L} \\
\text { - } \text { Outdoor pools: } 0.8-3 \mathrm{mg} / \mathrm{L} \\
\text { - } \quad \text { Pools }\left(\mathrm{T}>35^{\circ} \mathrm{C}\right): 2-3 \mathrm{mg} / \mathrm{L}\end{array}$ & $\begin{array}{l}\text { - Indoor pools: } 0.5 \mathrm{mg} / \mathrm{L} \\
\text { - Outdoor pools: } 1 \mathrm{mg} / \mathrm{L}\end{array}$ & - $7.2-7.8$ & (Canada, 2016) \\
\hline Germany & $\begin{array}{l}\text { - Pools: } 0.3-0.6 \mathrm{mg} / \mathrm{L} \\
\text { - Spa (hot tubs): } 0.7-1 \mathrm{mg} / \mathrm{L}\end{array}$ & - $\quad 0.2 \mathrm{mg} / \mathrm{L}$ & - $6.5-7.6$ & (Germany, 2012) \\
\hline Spain & - $0.4-1.5 \mathrm{mg} / \mathrm{L}$ & & - $6.8-8.0$ & (Spain, 2007) \\
\hline $\begin{array}{l}\text { Australia } \\
\text { (Queensland) }\end{array}$ & $\begin{array}{l}\text { - } \text { Indoor pools: }>1.5 \mathrm{mg} / \mathrm{L} \\
\text { - } \text { Heated indoor pools }\left(>26{ }^{\circ} \mathrm{C}\right):>2 \mathrm{mg} / \mathrm{L} \\
\text { - } \text { Outdoor pools: }>1.5 \mathrm{mg} / \mathrm{L} \\
\text { - } \text { Heated outdoor pools }\left(>26{ }^{\circ} \mathrm{C}\right):>3 \mathrm{mg} / \mathrm{L} \\
\text { - } \quad \text { Spa }\left(35-40{ }^{\circ} \mathrm{C}\right):>3 \mathrm{mg} / \mathrm{L}\end{array}$ & $\begin{array}{l}\text { - } 1 \mathrm{mg} / \mathrm{L} \text { (not exceed half } \\
\text { the total chlorine } \\
\text { concentration) }\end{array}$ & - $7.2-7.8$ & (Government, 2004) \\
\hline France & $\begin{array}{l}\text { - Pools (bleach or chlorine gas): } 0.4-1.4 \text { or } 0.3-0.6 \\
\mathrm{mg} / \mathrm{L}^{\mathrm{b}} \\
\text { - Pools (isocyanuric acid derivatives): } 2-4 \mathrm{mg} / \mathrm{L}\end{array}$ & - $\quad 0.6 \mathrm{mg} / \mathrm{L}$ & $\begin{array}{l}\text { - Pools: 6.9-7.7 } \\
\text { - Whirlpools: } 6.9-8.2\end{array}$ & $\begin{array}{l}\text { (ANSES, } \\
2014)\end{array}$ \\
\hline London & - $1-2 \mathrm{mg} / \mathrm{L}$ & & & (WHO, 2006) \\
\hline Italy & - $0.6-1.2 \mathrm{mg} / \mathrm{L}$ & & & (WHO, 2006) \\
\hline Switzerland & $\begin{array}{l}\text { - Public and semi-public pools: }<3 \mathrm{mg} / \mathrm{L} \\
\text { - Public and semi-public hot tubs: }<5 \mathrm{mg} / \mathrm{L}\end{array}$ & - $0.2 \mathrm{mg} / \mathrm{L}$ & $\begin{array}{l}\text { - Chlorine disinfectants: } \\
\text { 7.2-7.8 } \\
\text { - Bromine-based or other } \\
\text { non-chlorine } \\
\text { disinfectants: } 7.2-8.0\end{array}$ & (WHO, 2006) \\
\hline Singapore & - $1-3 \mathrm{mg} / \mathrm{L}$ & & - $7.2-7.8$ & (NEA, 2005) \\
\hline China & - $\quad 0.3-0.5 \mathrm{mg} / \mathrm{L}$ & & $\begin{array}{l}\text { - Artificial pools: } 6.8-8.5 \\
\text { - Natural pools: } 6.0-9.0\end{array}$ & (China, 1996) \\
\hline
\end{tabular}

Note:

${ }^{a}$ MCL means maximum contaminant level.

$\mathrm{b}$ The mandatory range of chlorine is $0.4-1.4 \mathrm{mg} / \mathrm{L}$. The range can be $0.3-0.6 \mathrm{mg} / \mathrm{L}$ when the hygiene conditions of pools are met. 
Table 2.5 THM concentrations in SPWs

\begin{tabular}{|c|c|c|c|c|c|c|c|}
\hline \multirow{2}{*}{ Country } & \multicolumn{5}{|c|}{ THM concentration $(\mu \mathrm{g} / \mathrm{L})$} & \multirow{2}{*}{ Pool type } & \multirow{2}{*}{ Reference } \\
\hline & $\mathrm{CHCl}_{3}$ & $\mathrm{CHCl}_{2} \mathrm{Br}$ & $\mathrm{CHClBr}_{2}$ & $\mathrm{CHBr}_{3}$ & THMs & & \\
\hline \multirow[t]{6}{*}{ U.S. } & $71(25-207)$ & $4(1-28)$ & $4(1-10)$ & $<1$ & $80(26-213)$ & 23 indoor $(\mathrm{Cl})$ & (Kanan et al., 2015) \\
\hline & $\sim 25$ & $<\mathrm{LOD}$ & $\sim 2$ & $<\mathrm{LOD}$ & & 1 indoor $(\mathrm{Cl})$ & (Weng and Blatchley, 2011) \\
\hline & $63(16-138)$ & & $6(1-35)$ & $7(1-55)$ & $78(19-146)$ & 11 indoor $(\mathrm{Cl})$ & (Weaver et al., 2009) \\
\hline & $80-130$ & & & & & 4 indoor $(\mathrm{Cl})$ & (Li and Blatchley, 2007) \\
\hline & $70-140$ & & & & & 2 outdoor $(\mathrm{Cl})$ & (Li and Blatchley, 2007) \\
\hline & $81(12-282)$ & & 2 (N.D.-10) & 1 (N.D.-32) & $77(33-282)$ & 1 indoor $(\mathrm{Cl})$ & (Afifi and Blatchley Iii, 2015) \\
\hline \multirow[t]{4}{*}{ Canada } & & & & & $44(18-114)$ & 15 indoor $(\mathrm{Cl})$ & (Simard et al., 2013) \\
\hline & & & & & $98(12-311)$ & 39 outdoor $(\mathrm{Cl})$ & (Simard et al., 2013) \\
\hline & $18-80$ & & & & & 8 indoor $(-)$ & (Lévesque et al., 2000) \\
\hline & $29(13-46)$ & & & & $29(13-46)$ & 2 indoor $(\mathrm{Cl})$ & (Catto et al., 2012) \\
\hline \multirow[t]{7}{*}{ Germany } & & & & & $39(5-125)$ & $\begin{array}{l}2 \text { outdoor } \\
\text { (TCCA) }\end{array}$ & (Zwiener et al., 2007) \\
\hline & & & & & 21 & 1 indoor $(\mathrm{Cl})$ & (Glauner et al., 2005b) \\
\hline & & & & & $35-47$ & 2 outdoor $(\mathrm{Cl})$ & (Glauner et al., 2005b) \\
\hline & $18(7-25)$ & & & & & 1 indoor $(\mathrm{Cl})$ & (Erdinger et al., 2004) \\
\hline & $23(21-27)$ & $3(2-3)$ & $<0.5$ & $<0.4$ & $27(25-30)$ & 3 indoor $(-)$ & (Cammann and Hübner, 1993) \\
\hline & $3-28$ & $0.7-6$ & $0.03-7$ & $0.02-0.8$ & & Indoor (-) & (Cammann and Hübner, 1995) \\
\hline & & & & & $14(6-43)$ & Indoor (-) & (Schmalz, 2012) \\
\hline \multirow[t]{6}{*}{ Spain } & $15(8-21)$ & $14(9-27)$ & $13(7-23)$ & $7(3-17)$ & $50(35-75)$ & 1 indoor $(\mathrm{Cl})$ & (Richardson et al., 2010) \\
\hline & $<0.3$ & $<0.7$ & $2(2-3)$ & $57(52-64)$ & $60(54-67)$ & $\begin{array}{l}1 \text { indoor } \\
(\mathrm{BCDMH})\end{array}$ & (Richardson et al., 2010) \\
\hline & $13.7 \pm 7.3$ & $1.4 \pm 0.7$ & $0.5 \pm 0.5$ & $0.3 \pm 0.7$ & $15.8 \pm 7.2$ & 20 indoor $(-)$ & (Marina et al., 2009) \\
\hline & & & & & $45.4 \pm 7.3$ & 1 indoor $(\mathrm{Cl})$ & (Kogevinas et al., 2010) \\
\hline & $15(9-20)$ & $14(9-25)$ & $13(7-23)$ & $7(3-16)$ & 50 & 1 indoor $(\mathrm{Cl})$ & (Lourencetti et al., 2012) \\
\hline & $<0.3$ & $<0.6$ & $2(2-3)$ & $60(52-61)$ & 63 & $\begin{array}{l}1 \text { indoor } \\
(\mathrm{BCDMH})\end{array}$ & (Lourencetti et al., 2012) \\
\hline \multirow[t]{4}{*}{ France } & $<0.3$ & $0.3(0.1-1.1)$ & $19(3-64)$ & $\begin{array}{l}300 \\
931)\end{array}$ & $\begin{array}{l}320 \\
996)\end{array}$ & $\begin{array}{l}8 \text { indoor } \\
\text { (seawater) }\end{array}$ & (Parinet et al., 2012) \\
\hline & $67(47-82)$ & $9(5-12)$ & $3(1-5)$ & $1(1-2)$ & $80(64-98)$ & 3 indoor $(\mathrm{Cl})$ & (Villanueva et al., 2007b) \\
\hline & 70 & 8 & 2 & 0.6 & 80 & 1 outdoor $(\mathrm{Cl})$ & (Manasfi et al., 2016) \\
\hline & N.D. & N.D. & $4(2-5)$ & $66(49-87)$ & $70(50-92)$ & $\begin{array}{l}3 \text { indoor } \\
\text { (seawater) }\end{array}$ & (Manasfi et al., 2016) \\
\hline Australia & $76(65-84)$ & $2(2-3)$ & $0.3-0.4$ & $<0.1$ & $79(67-87)$ & 1 outdoor $(\mathrm{Cl})$ & (Yeh et al., 2014) \\
\hline
\end{tabular}




\begin{tabular}{|c|c|c|c|c|c|c|c|}
\hline Italy & $33.2 \pm 24.6$ & $4.2 \pm 1.3$ & $1.9 \pm 2.0$ & $0.4 \pm 0.5$ & $39.8 \pm 21.7$ & 5 indoor $(\mathrm{Cl})$ & (Fantuzzi et al., 2001) \\
\hline Ireland & $88-116$ & $17-18$ & & & $105-133$ & 3 indoor $(\mathrm{Cl})$ & (Stack et al., 2000) \\
\hline England & $\begin{array}{l}121 \\
212)\end{array}$ & $8(3-23)$ & $3(1-7)$ & $1(1-2)$ & $\begin{array}{l}132 \\
223)\end{array}$ & 8 indoor $(\mathrm{Cl})$ & $\begin{array}{l}\text { (Chu and Nieuwenhuijsen, } \\
2002)\end{array}$ \\
\hline Korea & $\begin{array}{l}21 \quad \text { (N.D.- } \\
46)\end{array}$ & 2 (N.D. -7) & N.D. & N.D. & 23 & $\begin{array}{l}86 \text { indoor (three } \\
\text { disinfectants) }\end{array}$ & (Lee et al., 2010) \\
\hline
\end{tabular}

Note: ND: not detectable; LOD: limit of detection.

Table 2.6 HAA concentrations in SPWs

\begin{tabular}{|c|c|c|c|c|c|c|c|c|c|c|c|c|}
\hline \multirow{2}{*}{ Country } & \multicolumn{10}{|c|}{ HAA concentration $(\mu \mathrm{g} / \mathrm{L})$} & \multirow{2}{*}{ Pool type } & \multirow[b]{2}{*}{ Reference } \\
\hline & MCAA & MBAA & DCAA & TCAA & BCAA & DBAA & BDCAA & DBCAA & TBAA & HAAs & & \\
\hline \multirow[t]{4}{*}{ U.S. } & & & $\begin{array}{l}700 \\
(310- \\
1330)\end{array}$ & $\begin{array}{l}680 \\
(370- \\
1140)\end{array}$ & & & & & & $\begin{array}{l}1442 \\
(800- \\
2430)\end{array}$ & 6 outdoor $(\mathrm{Cl})$ & (Wang et al., 2014) \\
\hline & & & $\begin{array}{l}727(50- \\
2040)\end{array}$ & $\begin{array}{l}890(20- \\
2970)\end{array}$ & & & & & & $\begin{array}{l}1613 \\
(70- \\
3980)\end{array}$ & 6 indoor $(\mathrm{Cl})$ & (Wang et al., 2014) \\
\hline & & & $\begin{array}{l}450(50- \\
750)\end{array}$ & $\begin{array}{l}330(40- \\
530)\end{array}$ & & & & & & $\begin{array}{l}1067 \\
(690- \\
1360)\end{array}$ & 3 spa $(\mathrm{Cl})$ & (Wang et al., 2014) \\
\hline & & $\begin{array}{l}2 \text { (MRL- } \\
5)\end{array}$ & $\begin{array}{l}777(52- \\
6787)\end{array}$ & $\begin{array}{l}437(76- \\
1925)\end{array}$ & $\begin{array}{l}21 \\
176)\end{array}$ & $8(1-25)$ & $\begin{array}{l}34 \\
110)\end{array}$ & $6(1-32)$ & & $\begin{array}{l}1541 \\
(172- \\
9005)\end{array}$ & 23 indoor $(\mathrm{Cl})$ & (Kanan et al., 2015) \\
\hline \multirow[t]{3}{*}{ Canada } & & & & & & & & & & $\begin{array}{l}364 \\
(104- \\
1195)\end{array}$ & 15 indoor $(\mathrm{Cl})$ & (Simard et al., 2013) \\
\hline & & & & & & & & & & $\begin{array}{l}808 \\
(155- \\
2224)\end{array}$ & $\begin{array}{l}39 \text { outdoor } \\
(\mathrm{Cl})\end{array}$ & (Simard et al., 2013) \\
\hline & & & $\begin{array}{l}93(48- \\
192)\end{array}$ & $\begin{array}{l}10 \\
191)\end{array}$ & $2(1-3)$ & & $\begin{array}{l}15 \quad(< \\
\text { LOD-24) }\end{array}$ & & & $\begin{array}{l}218 \\
(111- \\
390)\end{array}$ & 2 indoor $(\mathrm{Cl})$ & (Catto et al., 2012) \\
\hline \multirow[t]{4}{*}{ Germany } & & & & $\begin{array}{l}206 \\
(175- \\
245)\end{array}$ & & & $1(1-2)$ & $<0.5$ & & & 3 indoor $(-)$ & $\begin{array}{ll}\text { (Cammann and } \\
\text { Hübner, 1993) }\end{array}$ \\
\hline & $\begin{array}{l}26 \\
81)\end{array}$ & $\begin{array}{l}0.3 \\
0.5-3)\end{array}$ & $\begin{array}{l}23 \\
192)\end{array}$ & $\begin{array}{l}42 \\
199)\end{array}$ & & $\begin{array}{l}0.6(0.2- \\
8)\end{array}$ & & & & & Indoor & $\begin{array}{l}\text { (Stottmeister and } \\
\text { Naglitsch, 1996) }\end{array}$ \\
\hline & $\begin{array}{l}32 \\
174)\end{array}$ & $\begin{array}{l}0.2 \\
0.5-2)\end{array}$ & $9(2-27)$ & $\begin{array}{l}15 \\
45)\end{array}$ & & $\begin{array}{l}0.6(0.2- \\
5)\end{array}$ & & & & & hydrotherapy & $\begin{array}{l}\text { (Stottmeister and } \\
\text { Naglitsch, 1996) }\end{array}$ \\
\hline & $\begin{array}{l}26 \\
112)\end{array}$ & $\begin{array}{l}0.1 \\
0.5-2)\end{array}$ & $\begin{array}{l}132 \\
562)\end{array}$ & $\begin{array}{l}249 \quad(8- \\
887)\end{array}$ & & $\begin{array}{l}0.1(0.2- \\
1)\end{array}$ & & & & & outdoor & $\begin{array}{l}\text { (Stottmeister and } \\
\text { Naglitsch, 1996) }\end{array}$ \\
\hline
\end{tabular}




\begin{tabular}{|c|c|c|c|c|c|c|c|c|c|c|c|c|}
\hline & $2(1-5)$ & & $\begin{array}{l}19 \quad(4- \\
59)\end{array}$ & $\begin{array}{l}10 \quad(4- \\
21)\end{array}$ & $\begin{array}{l}2(<0.6- \\
4)\end{array}$ & $<1$ & $5(2-10)$ & & & $\begin{array}{l}39 \quad(11- \\
99)\end{array}$ & Indoor (-) & (Schmalz, 2012) \\
\hline \multirow[t]{4}{*}{ Spain } & $\begin{array}{l}20 \\
36)\end{array}$ & & $\begin{array}{l}71 \quad(60- \\
91)\end{array}$ & $\begin{array}{l}105(85- \\
125)\end{array}$ & & & & & & $\begin{array}{l}207 \\
(154- \\
251)\end{array}$ & 1 indoor $(\mathrm{Cl})$ & $\begin{array}{l}\text { (Cardador } \\
\text { Gallego, 2011) }\end{array}$ \\
\hline & $\begin{array}{l}26 \\
34)\end{array}$ & & $\begin{array}{l}154 \\
(135- \\
170)\end{array}$ & $\begin{array}{l}122(99- \\
146)\end{array}$ & & & & & & $\begin{array}{l}302 \\
(254- \\
350)\end{array}$ & 1 outdoor $(\mathrm{Cl})$ & $\begin{array}{ll}\text { (Cardador } & \text { and } \\
\text { Gallego, 2011) }\end{array}$ \\
\hline & $15-1000$ & & & $\begin{array}{l}1000- \\
1700\end{array}$ & & & 208-912 & $\begin{array}{l}<\text { LOD- } \\
62\end{array}$ & $\begin{array}{l}<\text { LOD- } \\
15\end{array}$ & $\begin{array}{l}1300- \\
3200\end{array}$ & $3(-)$ & $\begin{array}{l}\text { (Loos and Barceló, } \\
2001 \text { ) }\end{array}$ \\
\hline & 4 & N.D. & 45 & 155 & 11 & 2 & 61 & 33 & 19 & 330 & $1(-)$ & (Sarrión et al., 2000) \\
\hline \multirow[t]{3}{*}{ France } & $\begin{array}{l}15 \quad(1- \\
96)\end{array}$ & $\begin{array}{l}42 \\
155)\end{array}$ & $4(1-9)$ & $\begin{array}{l}22 \\
87)\end{array}$ & $\begin{array}{l}50 \\
216)\end{array}$ & $\begin{array}{l}234(11- \\
1089)\end{array}$ & $10(1-20)$ & $\begin{array}{l}92 \\
243)\end{array}$ & $\begin{array}{l}124 \\
428)\end{array}$ & $\begin{array}{l}594(84- \\
2233)\end{array}$ & $\begin{array}{l}8 \text { indoor } \\
\text { (seawater) }\end{array}$ & (Parinet et al., 2012) \\
\hline & N.D. & N.D. & 23 & 461 & 2 & 2 & 7 & 3 & N.D. & 498 & 1 outdoor $(\mathrm{Cl})$ & (Manasfi et al., 2016) \\
\hline & N.D. & N.D. & N.D. & N.D. & $4(4-5)$ & $\begin{array}{l}66 \quad(63- \\
72)\end{array}$ & N.D. & $3(3-4)$ & $\begin{array}{l}43 \\
53)\end{array}$ & $\begin{array}{l}116 \\
(109- \\
132)\end{array}$ & $\begin{array}{l}3 \text { indoor } \\
\text { (seawater) }\end{array}$ & (Manasfi et al., 2016) \\
\hline Australia & $\begin{array}{l}59 \quad(< \\
0.5-120)\end{array}$ & $<0.5$ & $\begin{array}{l}1001 \\
(230- \\
2400)\end{array}$ & $\begin{array}{l}1151 \\
(110- \\
2600)\end{array}$ & $<0.5$ & $<0.5$ & $\begin{array}{l}12 \\
0.5-22)\end{array}$ & $<0.5$ & -- & $\begin{array}{l}2204 \\
(366- \\
5126)\end{array}$ & $\begin{array}{l}7 \text { outdoor }+5 \\
\text { indoor }(\mathrm{Cl})\end{array}$ & (Yeh et al., 2014) \\
\hline Korea & & & $\begin{array}{l}68 \\
246)\end{array}$ & $\begin{array}{l}156(20- \\
636)\end{array}$ & & & & & & & $\begin{array}{l}86 \text { indoor } \\
\text { (three } \\
\text { disinfectant) }\end{array}$ & (Lee et al., 2010) \\
\hline \multirow[t]{2}{*}{ China } & & & $\begin{array}{l}81 \\
195)\end{array}$ & $\begin{array}{l}52 \\
98)\end{array}$ & & & & & & $\begin{array}{l}156(89- \\
332)\end{array}$ & 5 outdoor $(\mathrm{Cl})$ & (Wang et al., 2014) \\
\hline & & & $\begin{array}{l}34 \quad(5- \\
60)\end{array}$ & $\begin{array}{l}53 \quad(6- \\
90)\end{array}$ & & & & & & $\begin{array}{l}95 \quad(13- \\
133)\end{array}$ & 9 indoor $(\mathrm{Cl})$ & (Wang et al., 2014) \\
\hline
\end{tabular}

Note: ND: not detectable; LOD: limit of detection; MRL: minimum reporting levels. 


\subsection{Toxicity and carcinogenicity of DBPs}

A previous review paper has systematically assessed the toxicity and carcinogenicity of some DBPs, therefore we only make a brief summary in this section to avoid the repetition (Richardson et al., 2007). THMs have been intensively investigated over the past 40 years since they were discovered. Toxicity effect of THMs is associated with diseases such as bladder and colon cancer, asthma, irritation to the eyes and mucous membrane, and reproductive function (Aggazzotti et al., 2004; Erdinger et al., 1998; Goodman and Hays, 2008; Hamidin et al., 2008). Among four regulated THMs, chloroform and bromodichlormethane are considered to be nongenotoxic and genotoxic carcinogens, respectively, and both are classified as possibly carcinogenic to humans (2B) by IARC (Richardson et al., 2007). MCAA, DCAA, TCAA, BCAA, DBAA and TBAA are reported to be cytotoxic and mutagenic in Salmonella typhimurium (Kargalioglu et al., 2002). Among the five regulated HAAs, DCAA has solely been considered as a possibly human carcinogen (2B) although it shows its weak genotoxicity only at high concentrations (Richardson et al., 2007). There are few data showing the evidence of genotoxicity or carcinogenicity of chlorite. Of all the regulated DBPs by U.S. EPA, bromate is the most potent carcinogen species which is classified as $2 \mathrm{~B}$.

The toxicology data for the unregulated DBPs are not as much as those for the regulated ones, however more attention has been paid in recent years due to their more severe genotoxic or mutagenic activities in some assays. For example, I-THMs, which are formed at high levels in chloraminated water, show higher chronic 
cytotoxicity to $\mathrm{CHO}$ cells than their Cl- or Br-THMs (Plewa et al., 2008). Similarly, I-HAAs are more mutagenic in TA100 and more genotoxic in CHO cells than the Cl- or Br-HAAs (Plewa et al., 2004). In addition, Br-HAAs were more toxic than ClHAAs to CHO cells and DNA and the increase of halogen atom number per molecule decreases the toxicity (Giller et al., 1997; Plewa et al., 2002). The suffering of dermatitis by swimmers in brominated swimming pools has been also reported (Pardo et al., 2007). HANs with low $\mu \mathrm{g} / \mathrm{L}$ concentration levels account for only 3.4$6.6 \%$ of the total DBPs in the chlorine or ozone/chlorine or EGMO treated pools (Lee et al., 2010). However, HANs are highly reactive and able to damage DNA in mammalian cells in vitro (Richardson et al., 2007). In general, N-, Br-, I-DBPs are more toxic than Cl- and C-DBPs (Muellner et al., 2007b; Richardson et al., 2007). Extracts of SPWs showed genotoxic effects in Ames-Test, with HepG2 cells, with CHO cells in a comet assay (Glauner et al., 2005b; Liviac et al., 2010; Richardson, 2010). The main compounds responsible for these genotoxic effects are so far unknown. Therefore, a comprehensive and systematic toxicology database for individual DBP is essential for the overall toxicology assessment to real SPWs.

\subsection{Human exposure and risk assessment}

\subsubsection{Exposure assessment of DBPs by biomarkers}

There are three main exposure routes of DBPs into human bodies: 1) inhalation of volatile compounds or aerosolized solutes; 2) dermal absorption via skin; 3) ingestion of DBP-contaminated water. The exposure of DBPs in swimming pools 
has been evaluated by the measurement of the corresponding DBP concentrations in participates' body matrix, including exhaled air, urine, blood, and plasma, as biomarkers (Caro and Gallego, 2007; 2008; Erdinger et al., 2004; Nieuwenhuijsen et al., 2000).

\subsubsection{THMs}

Dermal absorption and inhalation are two main exposure routes of THMs (due to their percutaneous absorbable and volatile properties) during activities such as bathing, showering and swimming in indoor pools (Villanueva et al., 2007a; Villanueva et al., 2007b; Xu et al., 2002). A level III fugacity model demonstrated the similar exposure level for dermal contact and inhalation for chloroform however higher for dermal contact for Br-THMs (Dyck et al., 2011). Erdinger et al. (2004) found $2 / 3$ of chloroform in the blood was taken up via inhalation and $1 / 3$ via dermal absorption under the assumption that accidental swallowing of water by divers is negligible. Similarly, Lourencetti et al. (2012) assessed the THM exposure by two predetermined actions, i.e., bathing in the water without exercise, and standing beside the pools. It showed dermal absorption (by subtracting the exposures in these two cases) accounted for $\sim 40 \%$ of inhalation. The chemical property "highly volatile" of these compounds could be a fundamental interpretation for deeming inhalation as a major exposure route. For example, the vapour pressure of chloroform (196 mm $\mathrm{Hg}$ ) is two orders of magnitude higher than that of trichloroacetic acid (1 mm Hg), which is the most volatile species in the group of HAAs. 
The crucial exposure of THM by inhalation attracts the public awareness of THM concentration in the ambient air especially in an indoor environment with a relatively closed space. It is not surprising to observe a positive correlation between chloroform concentrations in SPWs and its corresponding ambient air in Figure 2.3, although some data were extracted randomly from different literature and therefore were independent of each other (Catto et al., 2012; Fantuzzi et al., 2001; Fantuzzi et al., 2010; Kogevinas et al., 2010; Lévesque et al., 2000; Lourencetti et al., 2012; Marina et al., 2009; Richardson et al., 2010). The chloroform concentrations obtained from an indoor public pool in Spain demonstrated an excellent correlation (Caro and Gallego, 2008). It indicates that an accurate prediction of air concentration based on its corresponding water concentration is possible since air sampling is more complicated. It should be noted that different regions inside the swimming pools may have extremely different air concentrations. The average air concentration of THMs in poolside $\left(58.0 \mu \mathrm{g} / \mathrm{m}^{3}\right)$ was more than two times of that in the reception area and engine room (26.1 and $25.6 \mu \mathrm{g} / \mathrm{m}^{3}$ ) (Fantuzzi et al., 2001). Similarly, Aprea et al. (2010) found that the air concentrations near the pool were higher than those in other places far from the pools (e.g., change rooms, passageways and offices). 


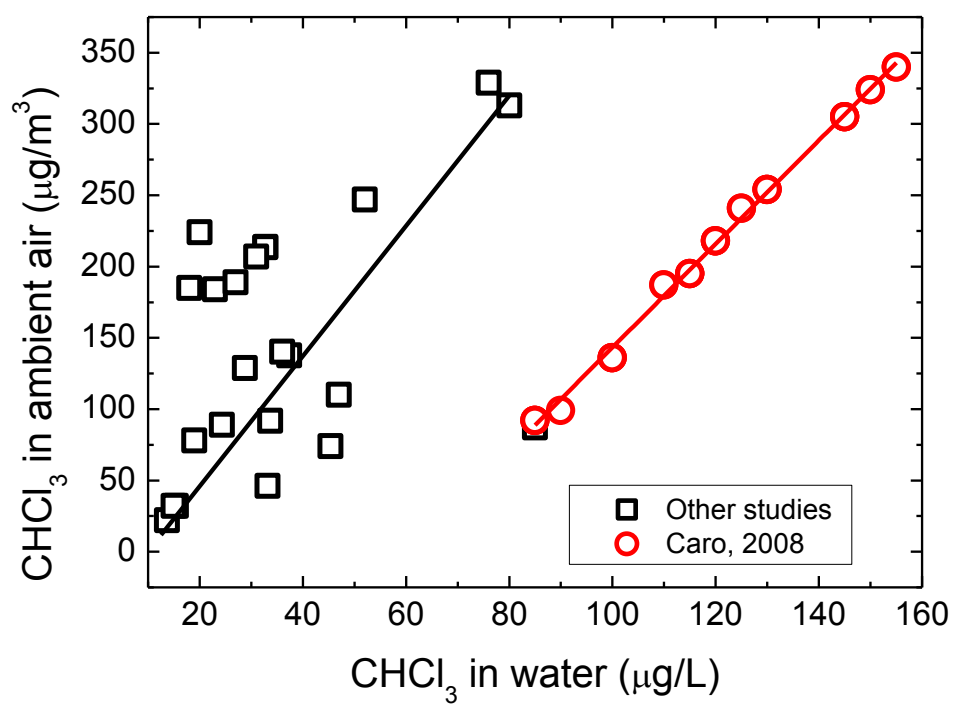

Figure 2.3 The relationship between $\mathrm{CHCl}_{3}$ concentrations in swimming pool water and in ambient air. Other studies include (Catto et al., 2012; Fantuzzi et al., 2001; Fantuzzi et al., 2010; Kogevinas et al., 2010; Lévesque et al., 2000; Lourencetti et al., 2012; Marina et al., 2009; Richardson et al., 2010).

The THM concentrations in alveolar air of swimmers or workers could be a good biomarker to evaluate THM exposure. The $\mathrm{CHCl}_{3}$ in swimmers' alveolar air had a quite wide concentration range of $\sim 10-350 \mu \mathrm{g} / \mathrm{m}^{3}$ (shown as black squares in Figure 2.4), due to a number of factors, including $\mathrm{CHCl}_{3}$ concentrations in water and air, exposure time, exercise intensity, coverage of pools (indoor/outdoor) and etc. (Catto et al., 2012; Fantuzzi et al., 2001; Fantuzzi et al., 2010; Kogevinas et al., 2010; Lévesque et al., 2000; Lourencetti et al., 2012; Marina et al., 2009; Richardson et al., 2010). Caro and Gallego have systematically investigated the THM exposure by human groups with different activities. The alveolar $\mathrm{CHCl}_{3}$ concentration increased 20 times for swimmers after $1 \mathrm{~h}$ swimming however only 8 times for workers after $2 \mathrm{~h}$ work shift (Caro and Gallego, 2008). Similarly, Fantuzzi et al. (2001) found that the alveolar $\mathrm{CHCl}_{3}$ concentrations of pool trainers were almost doubled compared to 
those worked as reception participants and engine room technicians (25.1 vs. 14.8 $\left.\mu \mathrm{g} / \mathrm{m}^{3}\right)$. The THM concentrations in alveolar air were highest for swimmers followed by those bathing in the pools without exercise and were lowest for those standing beside the pool outside the water (Lourencetti et al., 2012). It is worth noting that $\mathrm{CHCl}_{3}$ concentration in breathing zones of swimmers (i.e., boundary layer) was estimated to be 40 times higher than that in the ambient indoor air, which indicates that inhalation of $\mathrm{CHCl}_{3}$ by swimmers is more substantial than other non-swimmers (Chen et al., 2011). A good linear correlation between the $\mathrm{CHCl}_{3}$ concentrations in alveolar and ambient air as shown in Figure 2.4 further demonstrates the significance of inhalation for human exposure. The $\mathrm{CHCl}_{3}$ concentration in the alveolar air reached up to $124 \mu \mathrm{g} / \mathrm{m}^{3}$ after $1 \mathrm{~h}$ leisure activity, however $376 \mu \mathrm{g} / \mathrm{m}^{3}$ for competition swimmers (Lévesque et al., 2000). In addition, Lourencetti et al. (2012) found that physical activity enhanced THM exposure by comparing swimmers with swimming activities and volunteers with bathing only. Intensive exercises speed up swimmers' breathing and THM mass transfer, and therefore enhance swimmers' THM exposure via inhalation. In brominated pools, $\mathrm{CHBr}_{3}$ in swimmers' alveolar air reached up to $\sim 10 \mu \mathrm{g} / \mathrm{m}^{3}$ and showed a correlation with its corresponding ambient air concentrations as well (Lourencetti et al., 2012). 


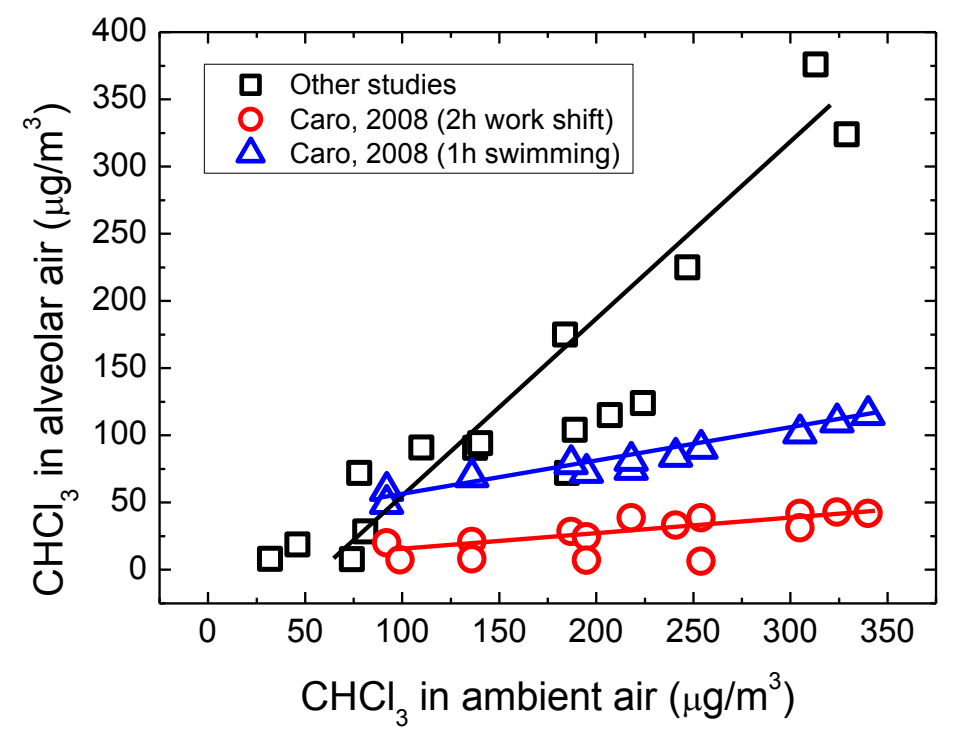

Figure 2.4 The relationship between $\mathrm{CHCl}_{3}$ concentrations in ambient air and alveolar air of swimmers. Other studies include (Catto et al., 2012; Fantuzzi et al., 2001; Fantuzzi et al., 2010; Kogevinas et al., 2010; Lévesque et al., 2000; Lourencetti et al., 2012; Marina et al., 2009; Richardson et al., 2010).

Urine is another biomarker for human exposure evaluation. Aprea et al. (2010) found the $\mathrm{CHCl}_{3}$ concentration in urine increased from 0.123 to $0.404 \mu \mathrm{g} / \mathrm{L}$ after some water activities. Caro et al. found the $\mathrm{CHCl}_{3}$ and $\mathrm{CHBrCl}_{2}$ concentrations in human urine are around 500 and $20 \mathrm{ng} / \mathrm{L}$ respectively before any exposure in swimming pool environment. The concentrations increased 3 times after $1 \mathrm{~h}$ swimming however only 2 times after $2 \mathrm{~h}$ work shift. Even after $4 \mathrm{~h}$ work shift, the concentration cannot reach as high as that after swimming (Caro and Gallego, 2007; 2008; Caro et al., 2007). Both swimmers and workers suffer THM exposure via inhalation, however, swimmers doing more strenuous exercises than workers inhale more air with higher THM concentration (just above the water level). In addition, swimmers exposed directly in SPWs experienced two additional exposure routes, i.e., dermal absorption and ingestion. Figure 2.5 shows the staffs working near the pool (e.g., monitors and 
pool guardians) experienced much higher exposure of $\mathrm{CHCl}_{3}$ than those working far away (e.g., receptionists and maintenance technician). It indicates that the relative position to the swimming pools where people stay significantly influences the THM exposure. Figure 2.5 also shows a good linear relationship between $\mathrm{CHCl}_{3}$ concentrations in SPWs and urine under similar conditions (e.g., activity and venue).

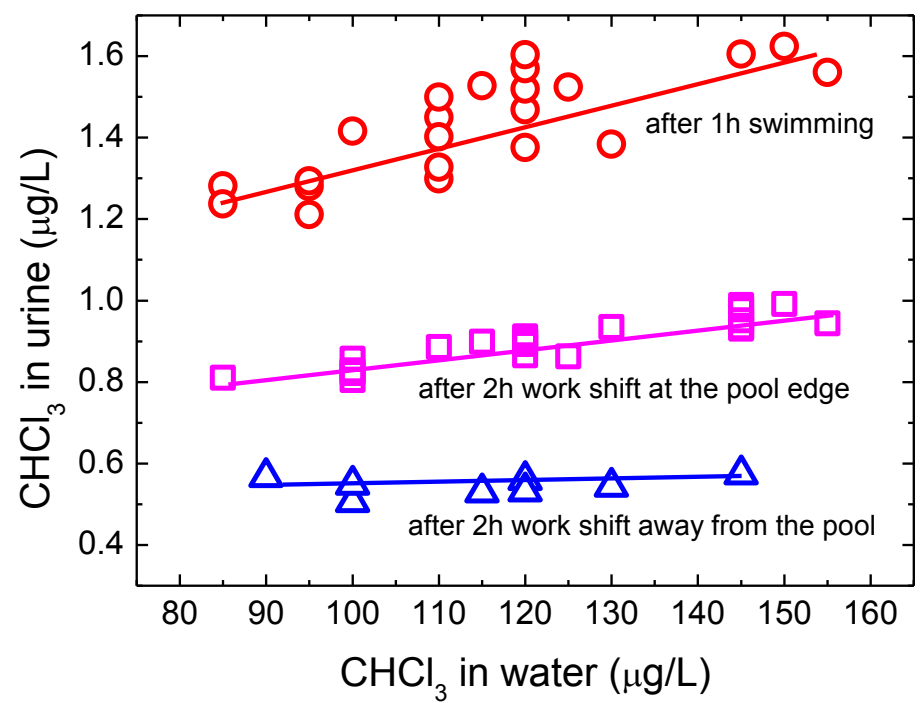

Figure 2.5 The relationship between $\mathrm{CHCl}_{3}$ concentrations in swimming pool water and in the urine of swimmers after $1 \mathrm{~h}$ swimming and workers after $2 \mathrm{~h}$ work shift. Data are from (Caro and Gallego, 2007; 2008).

In addition to the direct measurement of THMs, evaluation of micronuclei and DNA damage (comet assay), mutagenicity (ames assay) in human matrix (e.g., blood, urine), and its correlation with the THM concentration in alveolar air offers new insights of genotoxic effect assessment for swimmers exposed in SPWs (Kogevinas et al., 2010). Urine mutagenicity correlated proportionally with exhaled $\mathrm{CHBr}_{3}(p=$ 0.004) and the frequency change of micronucleated lymphocytes correlated with BrTHMs $(p \leq 0.05)$ after swimming, which reflects that Br-THMs are more genotoxic than chlorinated ones (Kogevinas et al., 2010). 
Cancer risk of THMs in SPWs was more than four times higher than that in DWs (Chowdhury, 2015). Swimming activity accounts for 93.9-94.2\% of the total risk of THM exposure. Swimmers exposed in THM-contaminated SPWs $(26.15-65.09 \mu \mathrm{g} / \mathrm{L})$ experienced the cancer risk up to $1.47 \times 10^{-3}$, which is classified as an unacceptable risk based on U.S. EPA. However, the risk for non-swimmers is in an acceptable level of $4.43 \times 10^{-5}$ (Panyakapo et al., 2008). A simulation study shows $>99 \%$ of the total cancer risk was attributed to $\mathrm{CHCl}_{3}$ inhalation $(<1 \%$ for dermal contact and oral ingestion) which further confirms inhalation to be the important exposure route (Chen et al., 2011).

\subsubsection{HAAs}

The more polar HAAs are less volatile and permeable to skin, and therefore have a higher potential of exposure by ingestion (Xu et al., 2002). HAA concentrations in SPWs as shown in Table 2.6 are generally much higher than those reported in DWs (Richardson et al., 2007), thus even a small amount of SPW ingestion may result in a large body intake. In 2010, Gallego's group developed a sensitive and straightforward method (headspace gas chromatography - mass spectrometry, HSGCMS) to detect HAAs in human urine with detection limits as low as ng/L, which established a technique foundation for the further investigation of HAA exposure among humans (Cardador and Gallego, 2010). Cardador and Gallego (2011) tested the urinary HAA concentrations of three groups of subjects to distinguish the respective role of three exposure pathways. They found that ingestion $(\sim 94 \%)$ 
outcompeted inhalation $(\sim 5 \%)$ and dermal absorption $(\sim 1 \%)$ and became a major exposure route. The finding that the urinary HAA concentration of the testers after 1 $\mathrm{h}$ swimming was more than an order of magnitude higher than that after $2 \mathrm{~h}$ work shift further validates ingestion as a more decisive exposure route than inhalation (Cardador and Gallego, 2011). More specifically, TCAA, DCAA and MCAA were present in swimmers' urine with concentrations of $\sim 4.4, \sim 2.3$, and $\sim 0.56 \mu \mathrm{g} / \mathrm{L}$ respectively after $1 \mathrm{~h}$ swimming in indoor pools. Font-Ribera et al. (2016) similarly reported the urinary TCAA increased from 3.9 to $12.3 \mu \mathrm{g} / \mathrm{L}$ after 40 min swimming in a chlorinated indoor pool. Urinary TCAA seems a better biomarker for ingestion exposure evaluation due to its longer biological half-life than other HAAs, e.g., DCAA (Bader et al., 2004; Kim et al., 1999).

Although the low volatility of HAAs, they are still detectable in air phase with a concentration range of 5-64 $\mu \mathrm{g} / \mathrm{m}^{3}$, owning to a great amount of aerosols (reflected by the humidity) in some indoor swimming pools (Pereira et al., 2012). Therefore, workers around the pools without touching the SPWs still have the high potential to suffer HAA exposure via inhalation of HAA-contaminated aerosols. Cardador and Gallego (2011) reported that DCAA and TCAA reached $\sim 300$ and $\sim 120 \mathrm{ng} / \mathrm{L}$ in the urine of workers after $2 \mathrm{~h}$ work shift in indoor swimming pools. These values were several times higher than those reported in outdoor pools and the exposure increased with time ( $2 \mathrm{~h}$ vs. $4 \mathrm{~h}$ as shown in Figure 2.6A, B). It indicates that sufficient ventilation in indoor pools is of high importance to ensure workers' safety. The linear relationship between HAA concentrations in human urine and SPWs as shown in Figure 2.6 offers the possibility to estimate the human HAA exposure by known 
HAA concentration in SPWs (Cardador and Gallego, 2011; Cardador and Gallego, 2010). Hence, HAA exposure by pool participants is concluded to be varied with a number of factors, e.g., HAA concentrations in SPWs, pool types (indoor or outdoor), exposure time, human activities, etc.
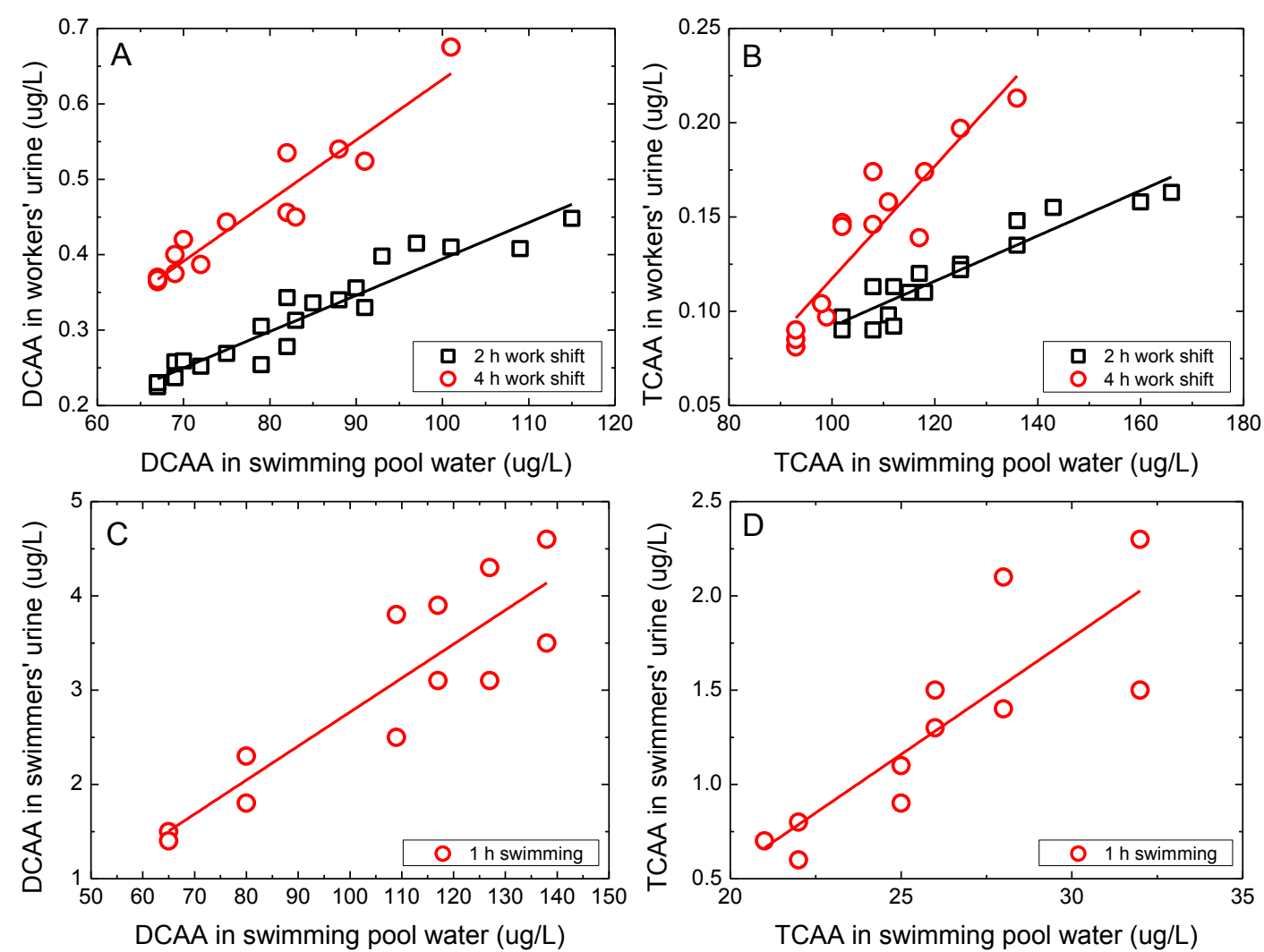

Figure 2.6 The relationship between HAA concentrations in SPWs and human urine. Data are from (Cardador and Gallego, 2011; Cardador and Gallego, 2010).

\subsubsection{Other DBPs}

Apart from these commonly mentioned regulated DBPs (like THMs and HAAs), other DBPs existed in SPWs may have the potential to be absorbed via human skin while swimming, which may be responsible for the health effects. For example, Xiao 
et al. (2012) newly identified some halo(nitro)phenols, among which 2,4dibromophenol, 2,4-dichlorophenol, and 2-bromophenol have the skin permeability of $0.031,0.021$, and $0.023 \mathrm{~cm} / \mathrm{h}$, respectively, in SPWs. Haloketones, as another prevalent group of chlorinated DBPs, have a skin permeation range of 0.024-0.043 $\mathrm{cm} / \mathrm{h}$, more than one order of magnitude higher than HAAs $(0.001-0.003 \mathrm{~cm} / \mathrm{h})$. Temperature is an important factor that affects the permeability of haloketones, i.e., the value triples as the temperature is increased from $20{ }^{\circ} \mathrm{C}$ to $40^{\circ} \mathrm{C}$ (Xu et al., 2002). These permeation studies may further promote the researchers' interest of exploring the content of these unregulated DBPs in the body matrix of pool participants which may contribute to certain health effects.

2.5.2 Epidemiological evidence and its potential relationship with pool activities

Health risks of pool activities are commonly assessed by the association with THMs, because of their sufficient data availability. Fantuzzi et al. (2010) reported that higher THM concentration in alveolar air $\left(>21 \mu \mathrm{g} / \mathrm{m}^{3}\right)$ was corresponding to higher risks of suffering from red eyes, itchy eyes, dyspnea/asthma and blocked nose, in a study of twenty indoor pools. Florentin et al. (2011) also mentioned that the increases of THM concentration and exposure duration may enhance the risk of colorectal cancer. Panyakapo et al. (2008) assessed the cancer risk of swimmers exposed to THMs to be $7.99 \times 10^{-4}$ in average which exceeds the acceptable level based on U.S. EPA standard. For non-swimmers, the average risk was only $2.19 \times 10^{-5}$ which is acceptable. Skin exposure of THMs during swimming accounts $94.2 \%$ of the total 
cancer risk and the rest is contributed by gastro-intestinal exposure while swimming, and skin and gastro-intestinal exposure from tap water. Similarly, Chowdhury (2015) found that the cancer risk via THM uptake is 4.06-6.64 times higher in SPWs than in DWs. Villanueva et al. (2007b) reported $23 \%$ of the total THM uptake from swimming but only $1 \%$ from DWs for pregnant women. In a study of 183 indoor swimming pools in Korea, Lee et al. (2009) assessed the lifetime cancer risk via THM inhalation during swimming to range between $7.77 \times 10^{-4}$ and $1.36 \times 10^{-3}$.

Apart from THMs, pool attendants, in fact, are exposed to a mixture of varieties of DBPs in aqueous and gaseous phases during pool activities. Therefore many studies reported the uncomfortable symptoms of pool participants and its potential relationship with the pool activity only. Fantuzzi et al. (2010) conducted a survey to record the declared health symptoms (e.g., ocular, cutaneous, asthma-related and other respiratory symptoms) among pool workers. It shows that workers in swimming pools suffered frequently from cold (65.4\%), sneezing $(52.6 \%)$, red eyes (48.9\%) and itchy eyes (44.4\%). Workers with the frequent touch of SPWs, e.g., lifeguards and trainers, were much easier to catch symptoms like red eyes, runny nose, voice loss and cold, than other employees in pool complexes (e.g., office and cafe). Villanueva and Font Ribera (2012) mentioned that pool participants had higher risks of suffering bladder cancer and asthma. Bernard et al. (2008) found that the increase of exposure time in pools increased the prevalence of asthma, respiratory allergies or airways inflammation for the adolescents as well. Villanueva et al. (2007a) similarly found that the risk of suffering bladder cancer increased with 
swimming activities and the duration of exposure to THM-contaminated water. However, there is still no explicit epidemiological evidence showing a direct association between swimming activities and reproductive outcomes (Villanueva and Font Ribera, 2012). It should be noted that the comprehensive identification and characterization of DBP mixture in SPWs are essential for a clear distinction on associations of health effects with DBPs.

\subsection{DBP treatment}

The high frequency of occurrence and high concentration of DBPs in SPWs indicates that the conventional pool water treatment system (flocculation-sand filtrationchlorination) cannot effectively remove DBPs or DBP precursors to acquire good water quality. Some novel control processes, e.g., membrane filtration, advanced oxidation processes, biodegradation, etc., aiming at DBP removal in SPWs are essential to secure the chemical safety of pool participates. In this section, we reviewed the most advanced DBP treatment approaches investigated in the past two decades.

\subsubsection{Membrane filtration}

Membrane has been widely used in the water and wastewater treatment and seawater desalination with its maturely developed technology and operation. The enhanced safety awareness towards DBPs in recent years promotes membrane filtration to be a potential technology for DBP treatment. Laboratory experiments reveal that DBP 
treatment by forward osmosis (FO), NF, and RO has achieved good results. Kong et al. (2014) first time used FO to deal with 9 HAAs and found that the rejection of each HAA reached more than $94.6 \%$ for the orientation of active layer facing the feed water using $1 \mathrm{~mol} / \mathrm{L} \mathrm{NaCl}$ as the draw solution. The rejection of five regulated HAAs reached $90-100 \%$ by a dense negatively charged NF membrane (Chalatip et al., 2009). Kimura et al. (2003) found 91-96\% of DCAA and TCAA were removed by NF/RO membranes. For THMs, Uyak et al. (2008) found that the rejection of total THMs reached as high as $95 \%$ under the conditions of interest by NF membranes. The rejection of individual THM followed the order of $\mathrm{CHClBr}_{2}>$ $\mathrm{CHCl}_{2} \mathrm{Br}>\mathrm{CHCl}_{3}$, attributed to the role of the molecular weight. $\mathrm{Xu}$ et al. (2005) found that high initial rejections of $\mathrm{CHCl}_{3}(>80 \%)$ and $\mathrm{CHBr}_{3}(>90 \%)$ by $3 \mathrm{NF} / \mathrm{RO}$ membranes was attributed to the effect of adsorption (hydrophobic-hydrophobic solute-membrane interactions) in addition to size exclusion. The rejections lowered down to $20-35 \%$ and $35-45 \%$ respectively at steady state conditions. Doederer et al. (2014) discovered an extremely wide range of rejections (i.e., 5-100\%) for 29 DBPs (e.g., THMs, I-THMs, HANs, HNMs, chloral hydrate, haloketones, and haloacetamides) by NF/RO membranes. These rejection data correlated well with the intrinsic DBP molecular properties (e.g., polar surface area, molecular volume, and etc.) and operational parameters (e.g., temperature and flux) by a multiple linear regression method.

In addition to bench-scale experiments, Klüpfel et al. (2011) attempted to apply an additional NF setup prior to the traditional sand filter process for a swimming pool. 
The rejection of DBPs (characterized as AOX) and DBP precursors (characterized as DOC) reached as high as $80 \%$ and $70 \%$, respectively. High rejections (86-94\%) for charged HAAs and low rejections (as low as 55\%) for neutral and low-molecularweight DBPs, e.g., $\mathrm{CHCl}_{3}, \mathrm{CHBrClCN}$, etc., were shown in a pilot-scale desalination plant (with 980,000 L/day, located in Carlsbad, California) equipped with a RO module (Agus and Sedlak, 2010). Fujioka et al. (2012) reviewed nnitrosodimethylamine removal based on the findings in some laboratory-, pilot- and full-scale RO plants and found significantly different rejections (negligible up to $86 \%$ ) caused by the varied operating parameters, e.g., feed solution temperature and $\mathrm{pH}$, ionic strength, and membrane permeate flux.

Filtration of DBP precursors is an effective method to minimize the DBP formation at source (Chellam, 2000; Sentana et al., 2010). Sentana et al. (2010) have discovered that NF membranes reduced the formation potential of HAAs and THMs (deemed as HAA-FP and THM-FP) by $>82 \%$. The rejection of THM and HAA precursors reached $77-96 \%$ and $75-92 \%$, respectively, by NF membranes under optimized conditions (Chellam, 2000). However, the high rejection of TOC (71-94\%) coupled with the extremely low rejection of bromide ions $(<10 \%)$ shifted Cl-DBPs to brominated ones upon chlorination to permeate water (Chellam, 2000).

The present studies demonstrate membrane filtration to be a potential candidate for DBP treatment in SPWs. However, the mechanisms governing these phenomena have not been systematically interpreted and fully understood. In addition, the 
challenges triggered by realistic SPW matrix or conditions should be considered before its real application. Klüpfel et al. (2011) found a polyamide membrane (XN45) exposed in a swimming pool with a chlorine concentration range of $0.6-2.2 \mathrm{mg} / \mathrm{L}$ experienced a decrease rejection of DOC and AOX after a period of filtration time. The constant chlorine exposure of membrane might damage the membrane material and affect its performance. The totally different DBP concentrations used in the filtration experiments may produce pretty different rejection due to some factors, i.e., concentration polarization, diffusion, and partitioning (Chalatip et al., 2009; Kimura et al., 2003; Uyak et al., 2008). It is essential to conduct the filtration experiments at concentrations of realistic interest. Fouling issue during long-time operation may occur, particularly for pools under frequent heavy usage (Barbot and Moulin, 2008). We should also pay special attention to the further treatment of DBP-contained retentate generated from the filtration process to make the effluent absolutely environmental-friendly.

\subsubsection{Advanced oxidation processes}

AOPs mainly refer to the processes employed $\mathrm{UV}, \mathrm{H}_{2} \mathrm{O}_{2}$ or $\mathrm{O}_{3}$, with hydroxyl radicals $(\cdot \mathrm{OH})$ as the active ingredient for the degradation of organic matter in the water environment. The application of AOPs in the SPW treatment has the high potential to eliminate DBPs and DBP precursors (Glauner et al., 2005a). Direct sunlight is a free natural source for DBP degradation in outdoor pools. Chen et al. (2010) attempted to investigate the photolysis of a variety of DBPs under natural solar irradiation and found the stability of DBPs generally followed the order of N- 
DBPs < C-DBPs, Br-, I-DBPs < Cl-DBPs, T-DBPs < D-DBPs < M-DBPs. Some THMs and HAAs showed high persistence to solar irradiation, i.e., 5-10\% of THM removal under $6 \mathrm{~h}$ solar exposure $\left(1150-1300 \mathrm{~W} / \mathrm{m}^{2}\right.$ irradiation intensities), indicating solar photolysis is insufficient for the degradation of these DBPs (Chen et al., 2010). It partly explains the high concentrations of DBPs, especially non-volatile HAAs, detected in some outdoor pools (see Table 2.5 and Table 2.6). The fate of $\mathrm{CH}_{2} \mathrm{I}_{2}, \mathrm{CH}_{2} \mathrm{ICl}$, and $\mathrm{CH}_{2} \mathrm{IBr}$ under natural solar photolysis demonstrated that one iodide was substituted by one chlorine (Jones and Carpenter, 2005).

DBPs exposed under UV or visible light in the presence of catalyst undergo good photocatalysis degradation. Lifongo et al. (2004) found that HAAs had better photocatalysis degradation under visible light $(\lambda>400)$ in the presence of $\mathrm{TiO}_{2}$, compared to photolysis only (without $\mathrm{TiO}_{2}$ ). HAAs with a higher number of halogens had higher degradation rate constants and almost no photolysis was observed for mono-halogen HAAs. Dehalogenation of HAAs was the proposed mechanism due to the halide ions detected during the reactions (for example, TCAA may degrade to form DCAA and chloride ions (Eq. (2.10))). In addition, HAAs under high temperatures underwent thermal degradation (Lifongo et al., 2004). Spangenberg is a pioneer researcher who has proposed two possible degradation mechanisms for TCAA: 1) photolysis by radical decarboxylation to form $\mathrm{CHCl}_{3}$ and $\mathrm{CO}_{2}$ (Eq. (2.11)); 2) pyrolysis by dehalogenation to form $\mathrm{HCl}$ and $\mathrm{CO}_{2}$ (Eq. (2.12)) (Spangenberg et al., 1996). Some potential metal catalysts (e.g. Cu, Fe, Mn, Ti, V, Mo and Zn) showed no direct promotion for HAA degradation (Lifongo et al., 2010). 


$$
\begin{gathered}
\mathrm{CCl}_{3} \mathrm{COOH} \underset{\mathrm{TiO}_{2}}{\longrightarrow} \mathrm{CHCl}_{2} \mathrm{COOH}+\mathrm{Cl}^{-} \\
\mathrm{CCl}_{3} \mathrm{COOH} \stackrel{\text { hv }}{\longrightarrow} \mathrm{CHCl}_{3}+\mathrm{CO}_{2} \quad \Delta \mathrm{H}_{\mathrm{R}}=-11.3 \mathrm{~kJ} / \mathrm{mol} \\
\mathrm{CCl}_{3} \mathrm{COOH}+\mathrm{H}_{2} \mathrm{O}+\frac{1}{2} \mathrm{O}_{2} \stackrel{\text { Heat }}{\longrightarrow} 3 \mathrm{HCl}+2 \mathrm{CO}_{2} \quad \Delta \mathrm{H}_{\mathrm{R}}=-486.4 \mathrm{~kJ} / \mathrm{mol}
\end{gathered}
$$

$\mathrm{UV} / \mathrm{H}_{2} \mathrm{O}_{2}$ based AOPs are widely used in the treatment of DBPs. Rudra et al. (2005) found 70 min UV irradiation completely degraded THMs except for $\mathrm{CHCl}_{3}(46 \%)$ with an initial concentration of $50 \mu \mathrm{g} / \mathrm{L}$ of each THM. The addition of $0.1 \% \mathrm{H}_{2} \mathrm{O}_{2}$ made a further contribution to $\mathrm{CHCl}_{3}$ degradation (92\%). However, only $10-12 \%$ of individual THM was removed in $0.1 \% \quad \mathrm{H}_{2} \mathrm{O}_{2}$ solution without $\mathrm{UV}$ exposure, indicating sole $\mathrm{H}_{2} \mathrm{O}_{2}$ to be a mild oxidizer. The $\mathrm{UV} / \mathrm{H}_{2} \mathrm{O}_{2}$ provides oxidation via $\cdot \mathrm{OH}$ generation by photolysis of $\mathrm{H}_{2} \mathrm{O}_{2}$, which is consistent with $\cdot \mathrm{OH}$ dependent degradation of Cl-DBPs (Eq. (2.14)) (Jo et al., 2011; Rudra et al., 2005). Jo et al. (2011) found that the removal of Br-THMs and HAAs reached more than $80 \%$ at a UV dose of $1200 \mathrm{~mJ} / \mathrm{cm}^{2}$ in the presence of $6 \mathrm{mg} / \mathrm{L} \mathrm{H}_{2} \mathrm{O}_{2}$ and the removal efficiency increased with the increased bromine number in the molecules, e.g., $\mathrm{CHBr}_{3}>$ $\mathrm{CHBr}_{2} \mathrm{Cl}$ and TBAA > DBAA. The degradation of Br-DBPs mainly rely on UV hydrolysis (Eq. (2.13), $\mathrm{X}$ refers to a halogen) in the $\mathrm{UV} / \mathrm{H}_{2} \mathrm{O}_{2}$ process, attributed to high UV absorption by Br-DBPs and enough energy from UV photon $(113 \mathrm{kcal} / \mathrm{mol}$ at $253.7 \mathrm{~nm}$ ) for the cleavage of $\mathrm{C}-\mathrm{Br}$ bond $\left(70.4 \mathrm{kcal} / \mathrm{mol}\right.$ for $\left.\mathrm{CHBr}_{3}\right)$ (Jo et al., 2011). Xiao et al. (2014) attempted to degrade I-THMs by UV254 irradiation. The I-THMs underwent a rapid first-order photodegradation process via cleavage of 
carbon-halogen bond, with reaction rate constants (in the range of 0.1-0.6 $\mathrm{min}^{-1}$ ) proportional to their corresponding molar extinction coefficients (an indicator of UV absorption). The end-product identification showed THMs were mainly conversed to hydrogen halide (92-109\% recovery) and also produced minor $\mathrm{HCOOH}(5 \%$ carbon conversion) as shown in Eqs. (2.15) and (2.16) (Xiao et al., 2014), which is similar to the observation by Kwok et al. (2004). The degradation rate constants of I-THMs in the $\mathrm{UV} / \mathrm{H}_{2} \mathrm{O}_{2}$ system increased to more than $2 \mathrm{~min}^{-1}$, indicating the $\cdot \mathrm{OH}$ promoted photolysis in addition to direct UV photolysis (Xiao et al., 2014). The combined UV radiation and ultrasound sonolysis (UV-US) showed greater degradation rate for TCAA than UV and US alone, attributed to dual effects of free radical excitation by UV and thermal degradation by the US (Wu et al., 2001).

$$
\begin{gathered}
\text { OH oxidation (C-H cleavage): } \mathrm{H}_{2} \mathrm{O}_{2} \stackrel{\text { hv }}{\longrightarrow} \cdot \mathrm{OH} \stackrel{\mathrm{R}_{3} \mathrm{C}-\mathrm{H}}{\longrightarrow} \mathrm{H}_{2} \mathrm{O}+\mathrm{R}_{3} \mathrm{C} \cdot(2.13) \\
\text { Direct UV photolysis (C-X cleavage) }: \mathrm{R}_{3} \mathrm{C}-\mathrm{X} \stackrel{\text { hv }}{\longrightarrow} \mathrm{R}_{3} \mathrm{C} \cdot+\mathrm{X} \cdot
\end{gathered}
$$

$$
\begin{gathered}
\text { Major reaction : } \mathrm{CHX}_{3}+\mathrm{H}_{2} \mathrm{O} \stackrel{\text { hv }}{\longrightarrow} \mathrm{CO}+3 \mathrm{HX} \\
\text { Minor reaction : } \mathrm{CHX}_{3}+2 \mathrm{H}_{2} \mathrm{O} \stackrel{\text { hv }}{\longrightarrow} \mathrm{HCOOH}+3 \mathrm{HX}
\end{gathered}
$$

Apart from regulated DBPs, some unregulated but potentially more toxic DBPs are degradable under UV photolysis. HNMs underwent homolysis or heterolysis to produce halides, nitrite, nitrate, and/or carbon dioxide under UV254 radiation (Fang et al., 2013). It is nice to observe that neutral to alkaline $\mathrm{pHs}$ which are typical for SPWs, contribute to HNM degradation due to their high UV molar absorptivity at 
these pHs. Two nitrogenous DBPs (N-DBPs), N-nitrosopyrrolidine and $\mathrm{N}$ nitrosopiperidine, were almost completely photodegraded (> 99\%) after only 5 min UV254 exposure (Xu et al., 2009). The N-N bond fission of these two compounds was proposed as the first photodegradation step followed by the further degradation to form aliphatic amines with low molecular weight. N-nitrosodimethylamine was degradable under UV with a reaction rate constant up to $0.36 \mathrm{~min}^{-1}$ (Sharpless and Linden, 2003). The addition of $\mathrm{H}_{2} \mathrm{O}_{2}$ slightly assisted or seriously hindered the nnitrosodimethylamine degradation due to the competing effects of light screening by $\mathrm{H}_{2} \mathrm{O}_{2}$ and the $\cdot \mathrm{OH}$ promoted n-nitrosodimethylamine degradation.

AOPs are also used to reduce the amount of DBP precursors, decrease the DBP formation potential and finally control the DBP levels in the treated water. The combined AOPs, i.e., $\mathrm{O}_{3} / \mathrm{UV}$ and $\mathrm{O}_{3} / \mathrm{H}_{2} \mathrm{O}_{2}$, illustrated better elimination of DBP precursors (using TOC, AOX-FP, THM-FP, and/or HAA-FP as indicators) than $\mathrm{O}_{3}$ and UV alone for SPW or surface water treatment (Chin and Bérubé, 2005; Glauner et al., 2005a). The removal efficiency of TOC, THM-FP and HAA-FP after $1 \mathrm{~h}$ $\mathrm{O}_{3} / \mathrm{UV}$ treatment reached $50 \%, 80 \%$ and $70 \%$ and reaction constants were $0.04,0.12$ and $0.15 \mathrm{~min}^{-1}$ respectively (Chin and Bérubé, 2005). Wang et al. (2000) found $\mathrm{UV} / \mathrm{H}_{2} \mathrm{O}_{2}$ an effective method for the remediation of a group of important DBP precursors, i.e., humic acids ( $\sim 90 \%$ removal within $1 \mathrm{~h}$ with a reaction rate constant of $\left.0.037 \mathrm{~min}^{-1}\right)$. 
In conclusion, considerable laboratory studies reviewed above have demonstrated the effectiveness of AOPs used for DBP degradation via the means of dehalogenation, decarboxylation, and others, depending on the specific AOP and DBP species. Nevertheless, there are still some concerns, limitations or contradictory findings we need to pay attention to. It is essential to optimize $\mathrm{H}_{2} \mathrm{O}_{2}$ amount dosed to the $\mathrm{UV} / \mathrm{H}_{2} \mathrm{O}_{2}$ system as an excess addition may result in a scavenging effect to the hydroxyl radical or light screening, eventually decreases the removal efficiency of DBPs or DBP precursors (Jo et al., 2011; Rudra et al., 2005; Sharpless and Linden, 2003; Wang et al., 2000). Humic acids, nitrate, chloride, and sulphate commonly detected in SPWs have the potential to affect the DBP photodegradation efficiency via shielding or activating $\cdot \mathrm{OH}$ (Chen et al., 2010; Xiao et al., 2014). In addition, the application of medium-pressure UV lamps in an indoor chlorinated public swimming pool even resulted in a significant increase of THMs, particularly $\mathrm{CHCl}_{3}$ and $\mathrm{CHBrCl}_{2}$ (Cassan et al., 2006). Therefore, the systematic understanding of real SPW characteristics and the fundamental reaction mechanisms are basic knowledge to ensure the effective AOP applications in real SPWs. Furthermore, the comprehensive identification of end-products (using mass balance) after AOPs is essential as DBPs are normally degraded to a variety of small weight compounds with unknown or even higher toxicity (Glauner et al., 2005b; Xu et al., 2009). Hence, it is far from enough to obtain a high degradation rate. An overall risk assessment for the degradation process is of great importance. 


\subsubsection{Biodegradation}

Biodegradation is another potential technique for the removal of DBPs in water systems. HAAs are shown to be biodegradable under conditions of interest (Chuang et al., 2011; Pluchon et al., 2013; Tung and Xie, 2009). Chlorine less than $0.3 \mathrm{mg} / \mathrm{L}$ and heterotrophic bacteria more than $10,000 \mathrm{HPC} / \mathrm{mL}$ were important water conditions for effective HAA removal (Chuang et al., 2011; Tung and Xie, 2009). Chuang et al. (2011) observed the removal of MCAA and DCAA reached up to $80 \%$ by sand filtration when the chlorine was less than $0.3 \mathrm{mg} / \mathrm{L}$. The bioactivity on the sand surface made a significant contribution to the HAA removal due to the development of biofilm under year-round high-temperature condition. However, we observed an average HAA concentration of $\sim 1000 \mu \mathrm{g} / \mathrm{L}$ in a public campus pool treated with sand filtration (Table 3.4). The contradictory findings might be explained by the difference of chlorine residuals in these two systems. The average chlorine concentration of $0.57 \mathrm{mg} / \mathrm{L}$ in the public pool (higher than the threshold 0.5 $\mathrm{mg} / \mathrm{L}$ ) may deactivate the corresponding bio-degraders and result in invalid HAA biodegradation (Chuang et al., 2011). The inverse relationship between heterotrophic bacteria and HAA concentrations under low chlorine concentration further emphasized the important role of bacteria for HAA degradation (Tung and Xie, 2009). Bacteria, e.g., S. natatoria and P. donghaensis, have been isolated and proven to be responsible for HAA degradation (Chuang et al., 2011). In addition, other parameters, including water temperature, retention time, the composition of organic matter, etc., affect HAA degradation simultaneously (Pluchon et al., 2013). On the contrary, biodegradation might not be favourable for THMs as a proportional 
relationship was observed between bacteria and THM concentration in some water distribution systems (Tung and Xie, 2009). The application of DBP biodegradation in SPWs still has a long way to go. The specific SPW matrix, in particular constant high chlorine (e.g., 1-3 mg/L), could be an unfavourable factor affecting the growth of some contributing bacteria. It is worthwhile to find robust bacteria which could tolerate the high chlorine conditions and promote the DBP removal as well.

\subsubsection{Thermal degradation and chemical reduction}

Lifongo et al. (2010) explored the thermal degradation of HAAs and found trihalogenated haloacetic acids (T-HAAs) were degraded by decarboxylation (as shown in Eq. (2.11) for TCAA), via C-C bond crack. The higher activation energy of decarboxylation reaction (C-C break) for TCAA compared to TBAA (151 vs. 139 $\mathrm{kJ} / \mathrm{mol} / \mathrm{k})$ was consistent to its lower reaction constant $\left(4.80 \times 10^{-10} \mathrm{vs} .7 .77 \times 10^{-8} \mathrm{~s}^{-1}\right.$, at $15^{\circ} \mathrm{C}$ ) (Lifongo et al., 2010). On the other hand, monohalogenated and dihalogenated haloacetic acids (M-HAAs, D-HAAs) were hydrolysed via oxygen attack to the $\alpha$-carbon of the molecules, producing glycollic and glyoxalic acids, and its corresponding hydrogen halide (as shown in Eq. (2.17) and (2.18), X represents halogens). The reaction rate for decarboxylation followed an order of T-HAAs > DHAAs > M-HAAs, as halogen attachment to the $\alpha$-carbon weakens the C-C bond. Zhang and Minear (2002) found the temperature-dependent decomposition of THAAs produce the corresponding THMs via decarboxylation.

$$
\mathrm{CH}_{2} \mathrm{XCOOH}+\mathrm{H}_{2} \mathrm{O} \rightarrow \mathrm{CH}_{2} \mathrm{OHCOOH}+\mathrm{HX}
$$




$$
\mathrm{CHX}{ }_{2} \mathrm{COOH}+\mathrm{H}_{2} \mathrm{O} \rightarrow \mathrm{CHOCOOH}+2 \mathrm{HX}
$$

Zero-valent iron $\left(\mathrm{Fe}^{0}\right)$ existing in the iron pipe system is an effective substance to degrade HAAs by dehalogenation (Hozalski et al., 2001; Tang et al., 2013). The debromination happened prior to dechlorination for the mixed chloro-bromo species, e.g., BDCAA, and therefore Br-HAAs were much easier to be removed than chlorinated ones (reaction rate constants followed $\mathrm{BDCAA}>\mathrm{CDBAA} \approx \mathrm{TBAA}>>$ TCAA as shown in Table 2.7) (Hozalski et al., 2001). Tang et al. (2013) found that DCAA and MCAA as the subsequent dehalogenation products of TCAA by $\mathrm{Fe}^{0}$ were completely biodegraded via a subsequent active carbon treatment process with a retention time of $10 \mathrm{~min}$. HAA dehalogenation was also achieved by an electrochemical reduction method using copper or gold as the electrode (Korshin and Jensen, 2001). Different from Br-HAAs (completely dehalogenated) Cl-HAAs produced notable MCAA whose direct reduction was difficult (Korshin and Jensen, 2001). DBPs other than HAAs, e.g., chloropicrin, trichloroacetonitrile, and dibromoacetonitrile, also underwent dehalogenation in the presence of sulphite as a reductant (Croue and Reckhow, 1989).

\subsubsection{Hybrid process}

The investigation of multi-stage DBP purification in SPWs is of high interests. For a municipal swimming pool in France, a hybrid process (hollow fiber ultrafiltration (UF) + granular activated carbon adsorption) was connected after a conventional SPW treatment system (coagulation-sand filtration-disinfection) (Barbot and Moulin, 
2008). This 18-month study illustrated that this hybrid process limited the combined chlorine (as an indicator of DBPs) to be lower than $0.35 \mathrm{mg} / \mathrm{L}$. Glauner et al. (2005b) used a two-step membrane filtration (UF followed by NF) to separate the compounds into three fractions with different ranges of molecular weight, i.e., $<200,200-1000,>$ $1000 \mathrm{~g} / \mathrm{mol}$. DBPs (characterized by AOX) with molecular weight less than 1000 $\mathrm{g} / \mathrm{mol}$ accounted for more than $80 \%$ of DBPs in both indoor and outdoor SPWs and the extract with lower molecular weight had higher genotoxicity. Therefore it is essential to use membranes with molecular weight cut-offs (MWCOs) less than 200 $\mathrm{g} / \mathrm{mol}$ to minimize its potential toxicity (Glauner et al., 2005b). Meanwhile, Glauner et al. (2005a) have attempted to apply UF or NF prior to AOPs for the treatment of SPWs and found that eliminations of DBPs and DBP precursors reached up to $80 \%$. The $\mathrm{Fe}^{0}$-active carbon treatment process contributed to TCAA dehalogenation by $\mathrm{Fe}^{0}$ and subsequent complete DCAA and MCAA (as the dehalogenated products of TCAA) biodegradation by active carbon within $10 \mathrm{~min}$ (Tang et al., 2013). The hybrid processes do have the potential to substitute or supplement the conventional SPW treatment to achieve better water quality. However, a full-scale application needs further validation, e.g., the stability of long-term operation, membrane foulinginduced side-effects. 
Table 2.7 HAA degradation rate constant

\begin{tabular}{|c|c|c|c|c|c|c|c|c|c|c|}
\hline \multicolumn{10}{|c|}{ HAA degradation rate constant $\left(\mathrm{min}^{-1}\right)$} & \multirow{2}{*}{ Reference } \\
\hline & MCAA & MBAA & DCAA & TCAA & BCAA & DBAA & BDCAA & DBCAA & TBAA & \\
\hline Solar photolysis & $3.48 \mathrm{E}-05$ & $1.87 \mathrm{E}-04$ & $1.18 \mathrm{E}-05$ & 4.00E-06 & $6.34 \mathrm{E}-05$ & $3.48 \mathrm{E}-04$ & $2.20 \mathrm{E}-05$ & $1.18 \mathrm{E}-04$ & $6.34 \mathrm{E}-04$ & $\begin{array}{l}\text { (Chen et al., } \\
2010)\end{array}$ \\
\hline $\begin{array}{l}\text { Photocatalysis by solar } \\
\text { light+ }+\mathrm{TiO}_{2}\end{array}$ & $1.15 \mathrm{E}-05$ & $7.68 \mathrm{E}-06$ & 4.61E-05 & $7.68 \mathrm{E}-05$ & $2.69 \mathrm{E}-05$ & $3.46 \mathrm{E}-05$ & & & $5.76 \mathrm{E}-05$ & $\begin{array}{l}\text { (Lifongo et al., } \\
\text { 2004) }\end{array}$ \\
\hline AOP by $\mathrm{H}_{2} \mathrm{O}_{2}+\mathrm{UV}$ & $3.48 \mathrm{E}-02$ & $6.60 \mathrm{E}-02$ & $3.00 \mathrm{E}-02$ & $1.74 \mathrm{E}-02$ & & $7.80 \mathrm{E}-01$ & & & $3.84 \mathrm{E}+00$ & (Jo et al., 2011) \\
\hline AOP by UV & & $1.26 \mathrm{E}-01$ & & & & $9.00 \mathrm{E}-01$ & & & $4.44 \mathrm{E}+00$ & (Jo et al., 2011) \\
\hline $\begin{array}{l}\text { Biodegradation by rapid } \\
\text { sand filtration }\end{array}$ & $1.70 \mathrm{E}-01$ & & $1.50 \mathrm{E}-01$ & & & & & & & $\begin{array}{l}\text { (Chuang et al., } \\
\text { 2011) }\end{array}$ \\
\hline $\begin{array}{l}\text { Reduction and } \\
\text { biodegradation by } \\
\mathrm{Fe}^{0}+\mathrm{BAC}\end{array}$ & $4.00 \mathrm{E}-01$ & & 4.00E-01 & $5.00 \mathrm{E}-01$ & & & & & & $\begin{array}{l}\text { (Tang et al., } \\
2013)\end{array}$ \\
\hline Reduction by $\mathrm{Fe}^{0}$ & & & & $1.33 \mathrm{E}-03$ & & & $1.77 \mathrm{E}-01$ & $2.38 \mathrm{E}-02$ & $2.35 \mathrm{E}-02$ & $\begin{array}{l}\text { (Hozalski et al., } \\
\text { 2001) }\end{array}$ \\
\hline $\begin{array}{l}\text { Thermal degradation at } \\
82-83^{\circ} \mathrm{C}\end{array}$ & 8.94E-05 & $1.39 \mathrm{E}-03$ & $2.69 \mathrm{E}-05$ & & $2.36 \mathrm{E}-04$ & $1.78 \mathrm{E}-04$ & & & & $\begin{array}{l}\text { (Lifongo et al., } \\
\text { 2010) }\end{array}$ \\
\hline
\end{tabular}




\subsection{Conclusion}

Previous research has mainly focused on the DBPs in the context of DWs subject to legal constraints by some global organizations or local government (e.g., EPA, WHO). Different from DWs, SPWs are more complicated and carry higher health risks due to the multifarious organic inputs from the bathers in addition to NOMs from source water. This chapter mainly reviewed the latest research progress on the occurrence, formation, and treatment of DBPs in the context of SPWs. More specifically, it provided comprehensive knowledge and information from multiple aspects, e.g., DBP regulations in different regions, formation mechanisms related with different disinfectants, precursors and other factors, toxicity and carcinogenicity, human exposure assessment reflected by biomarkers or epidemiological evidence, and latest approaches for the control and treatment of DBPs.

This critical review provided the potential directions of minimizing the DBP formation from the source and handling the formed DBPs. It demonstrated that chlorine/bromine stabilized disinfectants carry the potential to sustain the sufficient effective disinfection species in pools, especially for those exposed under sunlight. However, the formation of DBPs from these alternative disinfectants is still unclear. In addition, membrane filtration turned out to be a promising approach to control DBPs and DBP precursors. The underlying mechanisms have not been comprehensively understood and most laboratory-scale investigations ignored many significant realistic conditions. These unknown and unclear situations trigger our curiosities and passions for further investigations in the following chapters. 


\section{Chapter 3 An Insight of Disinfection By-Product Formation}

\section{by Alternative Disinfectants for Swimming Pool Disinfection under Tropical Conditions}

\subsection{Introduction}

Swimming is very popular in many tropical countries. There are more than 100,000 visitors annually in each public pool according to the local statistics in Singapore. The pool water safety has always been a top concern for the pool users and managers. Disinfection plays a predominant role to guarantee the microbiologically safe and hygienic water. However, disinfectants can react with water constituents of natural and anthropogenic origin to yield partly toxic DBPs (Fischer et al., 2012). For example, THMs are first identified and classified as possible carcinogens based on sufficient animal experimental data with pending further human evidence (EPA, 2005). THMs have also been statistically proven to associate with certain health concerns such as bladder and colon cancer, developmental effects and asphyxia among infants, and reproductive function (Aggazzotti et al., 2004; Hamidin et al., 2008; Hoffman et al., 2008; Villanueva et al., 2007a; Waller et al., 1998). Although Hrudey has pointed out that current human epidemiology data cannot causally link THM exposure with human cancer, it is still beneficial to explore ways to reduce the exposure due to their potential association (Hrudey, 2008; Hrudey et al., 2015). HAAs, as another prevalent class of DBPs, are also attracting concerns on their environmental occurrence, human exposure through dermal absorption, inhalation and ingestion, and high toxicity (Richardson, 2011; Richardson et al., 2007). There 
are some toxicological studies showing cytotoxic, genotoxic, mutagenic and teratogenic properties of HAAs to a variety of cells (Dad et al., 2013; Plewa et al., 2010; Procházka et al., 2015).

Increasing awareness of DBP formation and its toxicity makes the choice of disinfectants a novel and practical theme. Chlorine, chloramines, chlorine dioxide, and ozone are the most commonly used alternatives (Fischer et al., 2012). Chlorination with its residual chlorine is very popular, but the difficulty occurred when the permissible level of THMs in DWs was lowered from 100 to $80 \mu \mathrm{g} / \mathrm{L}$ in the U.S. (EPA, 1979; 1998). UV and ozone are alternatives for disinfection with limited halogenated DBP formation but no residual bactericidal ability. Chloramine combined with ozone formed the same kinds of halogenated DBPs as chlorine with ozone, but fewer in number and lower in concentration. However, compared to ozone as a sole disinfectant, some emerging DBPs, e.g. 1,1-dichloropropanone and dichloroacetaldehyde, are formed in high levels by these combined disinfection technologies (Richardson et al., 1999).

The phenomenon of high chlorine consumption and high DBP concentration in pools under sunlight disinfected by sodium hypochlorite $(\mathrm{NaClO})$ was observed in our preliminary survey (Section 3.3.1). It is a common challenge for pool operators to control the correct amount of chlorine to guarantee the microbial safety and minimize the DBP formation, because the chlorine measurement is periodic, i.e. three times per day. Two alternative organic-based disinfectants, TCCA and BCDMH, are 
known as stabilized chlorine/bromine. The fundamental principle of the stabilization property derives from two aspects: 1) The hydrolysis reactions as shown in Eqs. (2.7) and (2.8) are reversible and the equilibrium can be affected when $\mathrm{HClO} / \mathrm{HBrO}$ is partly consumed (Zwiener et al., 2007). 2) In the presence of sunlight UV with a wavelength range of 290-350 nm, $\mathrm{ClO}^{-}$with an absorption maximum of $290 \mathrm{~nm}$ undergoes photolysis to form chloride ion and oxygen. $\mathrm{HClO}$ also undergoes photolysis, but not as severe as $\mathrm{ClO}^{-}$, under the solar UV range (Feng et al., 2007). However, the bound forms of chlorine for TCCA, mainly including $\mathrm{HClCy}^{-}$and $\mathrm{Cl}_{2} \mathrm{Cy}^{-}$, under swimming pool conditions, have absorption maximum far below 290 $\mathrm{nm}$, thus are relatively stable under sunlight (Wojtowicz, 1996). Askins (2013) has discovered that $3 \mathrm{mg} / \mathrm{L} \mathrm{NaClO}$ as $\mathrm{Cl}_{2}$ can be degraded in less than 4 hours under direct sunlight exposure while the addition of $15 \mathrm{mg}$ cyanuric acid can keep chlorine available for $\sim 10$ hours, on a sunny day in April with an average temperature of 21 ${ }^{\circ} \mathrm{C}$ in Knightdale, North Carolina $\left(35^{\circ} 47^{\prime} 26^{\prime \prime} \mathrm{N} 78^{\circ} 29^{\prime} 31^{\prime \prime} \mathrm{W}\right)$. Compared with the most commonly used liquid $\mathrm{NaClO}$, TCCA and BCDMH in solid form offer the advantages of storage stability and dosing convenience, which are beneficial for some dosing systems in real applications. The consumer demand of TCCA for swimming pool disinfection is increasing by $8-10 \%$ annually (Tilstam and Weinmann, 2002). Compared to $\mathrm{NaClO}, \mathrm{BCDMH}$ has been proven as a more efficient bactericide, which is revealed by its better fecal coliform reduction in less time with an initial concentration of $1.9 \times 10^{6} \mathrm{CFU} / 100 \mathrm{~mL}$ under the same disinfectant dosage (Moffa et al., 2006). BCDMH also shows its advantage when it is used to deal with other bacteria, e.g. E. coli, E. aerogenes, P. aeruginosa, and 
polybacteria, at pH 8.5 (Nalepa, 2004). The better disinfection of BCDMH may be attributed to the fact that alkaline condition $(\mathrm{pH}>7.0)$ favors for more $\mathrm{HBrO}$ than $\mathrm{HClO}$ formation (Ginn et al., 1989). In terms of DBP formation, bromine reagents (e.g. BCDMH) generate brominated compounds dominantly, while chlorine reagents (e.g. $\mathrm{NaClO}$ ) mainly generate chlorinated ones in real swimming pools if bromide levels are below the instrument detection limit $(<0.1 \mathrm{mg} / \mathrm{L})($ Lourencetti et al., 2012; Richardson et al., 2010). However, whether the DBP concentration is below the regulation limit and whether the amount of the residual free chlorine is sufficient for pool disinfection (> $1 \mathrm{mg} / \mathrm{L}$ as $\mathrm{Cl}_{2}$ based on local regulation), especially for using the stabilized disinfectants, TCCA and BCDMH, have seldom been simultaneously taken into consideration in the existing literatures, even though both parameters are of great importance to the hygienic safety of swimmers.

Organic matters are another essential factor that controls the formation of DBPs. In SPWs, there are mainly two organic sources, NOM from filling water and body fluid from the swimmers (Chowdhury et al., 2014). Kanan and Karanfil (2011) have demonstrated the speciation of DBPs during the chlorination of NOM and BFA. In addition, the chlorination of individual organic precursor, e.g. urea, uric acid, creatinine, histidine, etc., and their formation mechanism of DBPs (e.g. THMs, chloramine and other nitrogenous compounds), have been reported (Blatchley III and Cheng, 2010; De Laat et al., 2011; Li and Blatchley, 2007; Lian et al., 2014). The general transformation pathways from these precursors to some regulated or unregulated DBPs have been discussed in some studies. For example, Hua and 
Reckhow (2008) have simplified the reaction pathways between NOM and chlorine/chloramine by means of the combination of oxidation, substitution and hydrolysis, leading to the formation of regulated THMs and HAAs. More specifically, urea, as a main species in body fluid, is chlorinated to yield trichloramine via a sequence of chlorine substitution and hydrolysis (De Laat et al., 2011; Li and Blatchley, 2007). Shah and Mitch (2012) have critically reviewed the formation pathways of nitrogenous DBPs and found these mechanisms are highly correlated with the specific precursors and disinfectants. One of the potential issues is that most of the current batch studies are conducted under certain matrix or conditions far from the real pool environment, e.g. the excess of chlorine, the unrealistic chlorine/precursor ratio, etc., which may result in the absence of some critical points and affect the final conclusion (see Section 3.3.5). In addition, the DBP formation and speciation by the stabilized disinfectants, e.g. TCCA and $\mathrm{BCDMH}$, under simulated pool water conditions has seldom been discussed in the existing literature.

Given these drawbacks of using $\mathrm{NaClO}$ in the tropical environment, and potential superiorities but lack of valid evidence of two stabilized disinfectants, TCCA and $\mathrm{BCDMH}$ were first time systematically investigated and compared to $\mathrm{NaClO}$ with respect to delivery and stabilization of chlorine/bromine, the reaction with precursors and formation of DBPs (including THMs and HAAs) under various simulated tropical climate pool conditions. 


\subsection{Materials and methods}

\subsubsection{Analytical methods}

HS-GCMS method was used for analysis of THMs based on a modified DIN 3840730: 2007-12 method. The THMs include chloroform, dibromochloromethane, bromodichloromethane and bromoform. Free chlorine in samples was quenched by $\sim 2 \mathrm{mg}$ ammonia chloride before $20 \mathrm{~mL}$ sample vials were filled headspace free. Sodium thiosulfate has also been used as the quench reagent and similar results were obtained. The water sample of $5 \mathrm{~mL}$ was transferred into a $10 \mathrm{~mL} \mathrm{GC}$ vial with PTFE-septa (gas-tight). One gram sodium sulfate was added to enhance the ionic strength in the aqueous phase to drive the targeted compounds into the gas phase. Before GC analysis, samples were shaken for $15 \mathrm{~min}$ at $60{ }^{\circ} \mathrm{C}$ (Agitator, MPSHeadspace Autosampler, Gerstel) to achieve equilibrium of the targeted compounds between gaseous and aqueous phase. Finally, $1 \mathrm{~mL}$ gas sample in the headspace was injected into the GC. Samples were analyzed by Agilent 7890A GC coupled with Agilent 5975C MS using a Restek-VMS column $(60 \mathrm{~m} \times 0.25 \mathrm{~mm}$ ID, $1.4 \mu \mathrm{m}$ film thickness). The oven temperature was held at $40{ }^{\circ} \mathrm{C}$ for $1 \mathrm{~min}$ and increased to $200{ }^{\circ} \mathrm{C}$ at a rate of $10{ }^{\circ} \mathrm{C} / \mathrm{min}$ and held for $1 \mathrm{~min}$. The total running time was $18 \mathrm{~min}$. The front inlet heater temperature was kept at $250{ }^{\circ} \mathrm{C}$. Injection was in a split mode (split ratio: 5:1) with $5.00 \mathrm{~min}$ solvent delay. The mass spectrometer was operated in electron ionization mode at $70 \mathrm{eV}$ with a scan range of $\mathrm{m} / \mathrm{z} 50-250 \mathrm{amu}$ and a scan rate of $6.6 \mathrm{scans} / \mathrm{sec}$. 
HAAs, including totally 9 compounds, chloroacetic acid, bromoacetic acid, dichloroacetic acid, bromochloroacetic acid, dibromoacetic acid, trichloroacetic acid, bromodichloroacetic acid, chlorodibromoacetic acid and tribromoacetic acid, were determined based on a modified EPA 552.3 method (see Figure 3.1). A $40 \mathrm{~mL}$ sample was adjusted to $\mathrm{pH} \leq 0.5$ by $2 \mathrm{~mL}$ concentrated sulfuric acid (98\%). The $2 \mathrm{~g}$ copper sulfate was added for better observation of phase separation and then $16 \mathrm{~g}$ sodium sulfate was added to achieve a saturated solution. The addition of $4 \mathrm{~mL}$ methyl tert-butyl ether (MTBE) was made followed by 30 min shaking to ensure sufficient extraction. For the derivatization of the HAAs to their methyl esters, $3 \mathrm{~mL}$ MTBE extract was incubated at $50{ }^{\circ} \mathrm{C}$ for 1.5 hours after the addition of $1 \mathrm{~mL}$ acidic methanol (10\% sulfuric acid in methanol). After quick cooling down by an ice bath, $4 \mathrm{~mL}$ saturated sodium bicarbonate was added for neutralization. Before venting the carbon dioxide, 2 minutes' shaking was done to ensure complete reaction. The vial was allowed to stand for 1 minute to separate aqueous and organic phases, then 1 $\mathrm{mL}$ of the methylated HAAs were transferred to a $2 \mathrm{~mL} \mathrm{GC}$ vial and were analyzed by an Agilent 6890 GC coupled with an Agilent 5973 MS equipped with a DB-5MS column (J \& W Scientific, 5\% phenyl - 95\% dimethyl polysiloxane, $30 \mathrm{~m} \times 0.25 \mathrm{~mm}$ ID, $0.25 \mu \mathrm{m}$ film thickness). The oven temperature was held at $35^{\circ} \mathrm{C}$ for $9 \mathrm{~min}$ and increased to $150{ }^{\circ} \mathrm{C}$ at a rate of $10{ }^{\circ} \mathrm{C} / \mathrm{min}$ and further increased to $250{ }^{\circ} \mathrm{C}$ at a rate of $20^{\circ} \mathrm{C} / \mathrm{min}$. The total running time was $25.5 \mathrm{~min}$. Carrier gas $(\mathrm{He})$ flowed at a constant rate of $0.6 \mathrm{~mL} / \mathrm{min}$. The transfer line, MS quad and source temperatures were kept at 280,150 and $300{ }^{\circ} \mathrm{C}$, respectively. A $1 \mu \mathrm{L}$ sample was injected for analysis in a splitless mode with 5.40 min solvent delay. The mass spectrometer was 
operated in EI mode at $70 \mathrm{eV}$ with a scan range of $\mathrm{m} / \mathrm{z} 50-300$ amu and a scan rate of $5.5 \mathrm{scans} / \mathrm{sec}$.

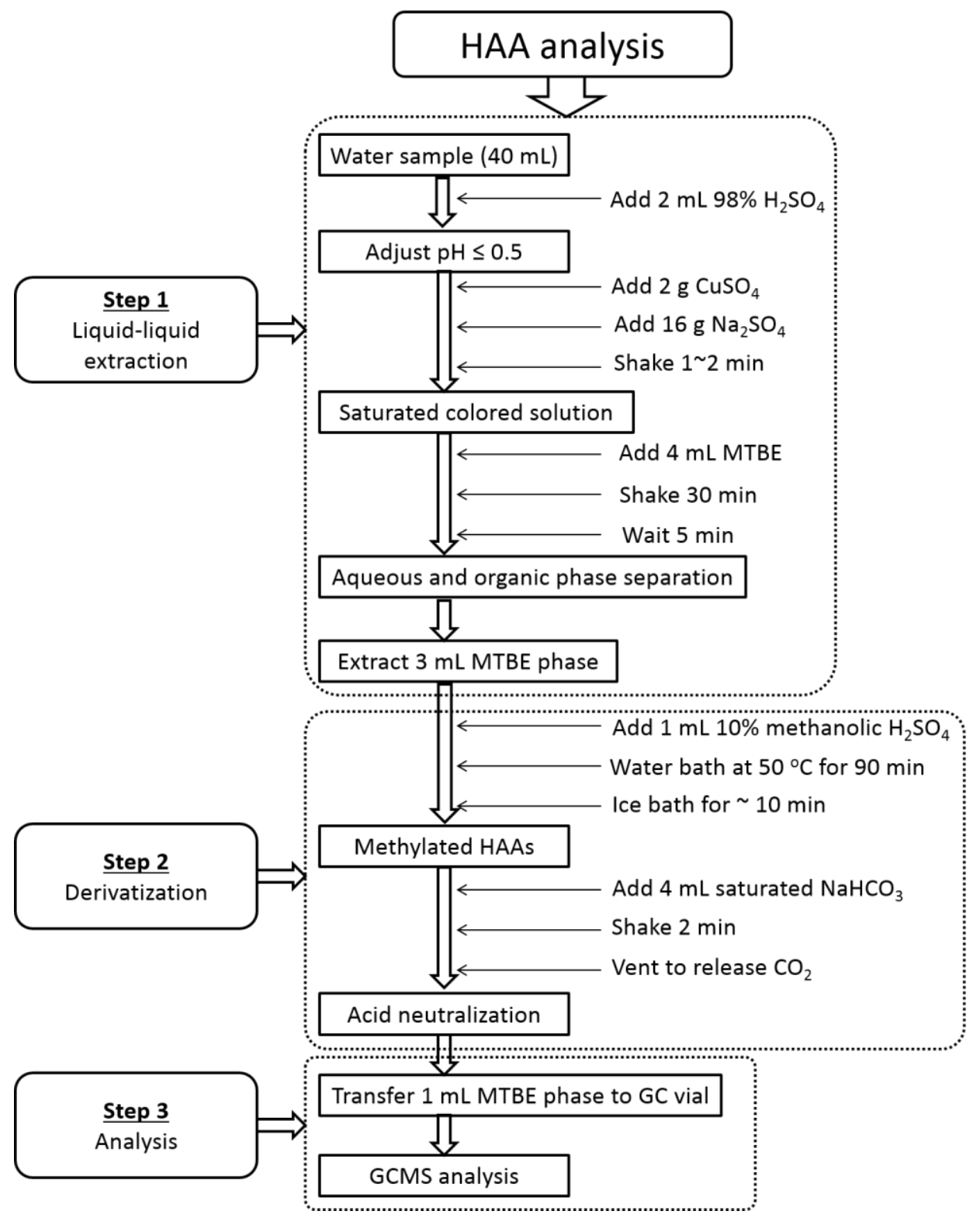

Figure 3.1 Flow chart of HAA analysis procedure (adapted from EPA 552.3)

THMs and HAAs were qualified by at least two qualifier ions and quantified by a quantifier ion. The method development and validation parameters with retention time, quantifier and qualifier ions, linearity, repeatability and reproducibility are 
listed in Table 3.1 and Table 3.2. The use of standards to do the calibration curves (7 points) and blanks to check the baseline of the detector were routinely performed before each batch of real sample measurements. Samples with known concentrations were intermittently used to check the performance of the detector during the unknown sample analysis (one known sample injection of every 10 unknown sample measurements).

Table 3.1 THM method development and validation parameters with retention time, quantifier and qualifier ions, linearity, repeatability and reproducibility ${ }^{\mathrm{a}}$

\begin{tabular}{lllllllll}
\hline Number & Substance & $\begin{array}{l}\text { Retention } \\
\text { Time } \\
(\mathrm{min})\end{array}$ & $\begin{array}{l}\text { Quantifier } \\
(\mathrm{m} / \mathrm{z})\end{array}$ & $\begin{array}{l}\text { Qualifier } \\
(\mathrm{m} / \mathrm{z})\end{array}$ & $\begin{array}{l}\text { Qualifier } \\
(\mathrm{m} / \mathrm{z})\end{array}$ & $\begin{array}{l}\text { Linearity } \\
\left(\mathrm{R}^{2}\right)^{\mathrm{b}}\end{array}$ & $\begin{array}{l}\text { Repeatability } \\
(\% \mathrm{RSD}, \mathrm{n}=3)^{\mathrm{c}}\end{array}$ & $\begin{array}{l}\text { Reproducibility } \\
(\% \mathrm{RS}, \mathrm{n}=3)^{\mathrm{d}}\end{array}$ \\
\hline 1 & $\mathrm{CHCl}_{3}$ & 8.60 & 83 & 85 & -- & 0.9999 & 5.0 & 5.9 \\
2 & $\mathrm{CHBrCl}_{2}$ & 10.50 & 83 & 85 & 129 & 1.0000 & 4.0 & 6.1 \\
3 & $\mathrm{CHBr}_{2} \mathrm{Cl}$ & 12.50 & 129 & 127 & -- & 1.0000 & 4.5 & 3.1 \\
4 & $\mathrm{CHBr}_{3}$ & 14.30 & 173 & 171 & -- & 0.9999 & 1.6 & 4.2 \\
\hline
\end{tabular}

Notes:

${ }^{\text {a }}$ The recoveries of THMs were within $99-109 \%$ by fortifying a known amount of standards to an unknown sample based on DIN 38407-30:2007-12.

b The linearity of calibration curves for each compound was determined by a series of 7-point concentration levels, $0.2,0.5,1,5,10,20$, and $50 \mathrm{ug} / \mathrm{L}$.

${ }^{\mathrm{c}}$ The repeatability was determined with triplicate of each calibration levels on the same day and was calculated by the average of relative standard deviation (RSD) of 7-point.

${ }^{d}$ The reproducibility was determined by checking the day-by-day stability of this method. Each standard (7-point) was analyzed in three consecutive days and average RSD of each compound was calculated.

Table 3.2 HAA method development and validation parameters with retention time, quantifier and qualifier ions, linearity, repeatability and reproducibility ${ }^{\mathrm{a}}$

\begin{tabular}{lllllllll}
\hline Number & Substance & $\begin{array}{l}\text { Retention } \\
\text { Time } \\
(\mathrm{min})\end{array}$ & $\begin{array}{l}\text { Quantifier } \\
(\mathrm{m} / \mathrm{z})\end{array}$ & $\begin{array}{l}\text { Qualifier } \\
(\mathrm{m} / \mathrm{z})\end{array}$ & $\begin{array}{l}\text { Qualifier } \\
(\mathrm{m} / \mathrm{z})\end{array}$ & $\begin{array}{l}\text { Linearity } \\
\left(\mathrm{R}^{2}\right)^{\mathrm{b}}\end{array}$ & $\begin{array}{l}\text { Repeatability } \\
(\% \mathrm{RSD}, \mathrm{n}=3)^{\mathrm{c}}\end{array}$ & $\begin{array}{l}\text { Reproducibility } \\
(\% \mathrm{RSD}, \mathrm{n}=3)^{\mathrm{d}}\end{array}$ \\
\hline 1 & MCAA & 5.56 & 108 & 77 & 59 & 0.9993 & 2.3 & 2.0 \\
2 & MBAA & 10.52 & 93 & 59 & -- & 0.9997 & 2.1 & 1.4 \\
3 & DCAA & 11.20 & 83 & 59 & -- & 0.9989 & 0.5 & 0.4 \\
4 & TCAA & 14.08 & 117 & 59 & -- & 0.9986 & 1.3 & 0.8 \\
5 & BCAA & 14.27 & 129 & 59 & -- & 0.9989 & 0.8 & 0.8 \\
6 & DBAA & 16.33 & 173 & 107 & -- & 0.9988 & 1.0 & 1.3 \\
7 & BDCAA & 16.43 & 163 & 59 & -- & 0.9984 & 1.9 & 7.5 \\
\hline
\end{tabular}




\begin{tabular}{lllllllll}
\hline 8 & DBCAA & 18.35 & 207 & 205 & -- & 0.9949 & 3.6 & 10.5 \\
9 & TBAA & 20.01 & 253 & 251 & -- & 0.9904 & 2.5 & 11.8 \\
\hline
\end{tabular}

Notes:

${ }^{a}$ The recoveries of HAAs were within $98-101 \%$ by fortifying a known amount of standards to an unknown sample based on EPA 552.3.

$\mathrm{b}$ The linearity of calibration curves for each compound was determined by a series of 7-point concentration levels, 2, 5, 10, 15, 20, 50, and $100 \mathrm{ug} / \mathrm{L}$.

${ }^{\mathrm{c}}$ The repeatability was determined with triplicate of each calibration levels on the same day and was calculated by the average of relative standard deviation (RSD) of 7-point.

$\mathrm{d}$ The reproducibility was determined by checking the day-by-day stability of this method. Each standard (7-point) was analyzed in three consecutive days and average RSD of each compound was calculated.

Free and combined chlorine/bromine were analyzed photometrically with the dipropyl-p-phenylenediamine (DPD) method (Spectroquant ${ }^{\circledR}$ chlorine test, EPA 330.5, US Standard Methods 4500-Cl2 G, and EN ISO 7393) immediately after sampling. We would like to highlight that the reported free chlorine for TCCA includes both $\mathrm{HClO}$ and $\mathrm{ClO}^{-}$, and bound forms of chlorine. Bound chlorine undergoes rapid hydrolysis during DPD measurement, thus actually represents potential free chlorine releasing $\mathrm{HClO}$ and $\mathrm{ClO}^{-}$on demand (Wojtowicz, 1996). The species distribution of TCCA was calculated and displayed in Figure 3.2 based on the relevant equilibrium information with solution $\mathrm{pH}$, measured free chlorine concentration, and cyanuric acid concentration and equilibrium constants (Wojtowicz, 1996). Additionally, our preliminary test showed the recovery of the chlorine in TCCA by DPD method reached $89 \%-110 \%$ under corresponding experimental conditions. The recovery was calculated by dividing the measured free chlorine concentration by theoretical free chlorine concentration. The theoretical value assumed all the chlorine elements in TCCA were HClO. Similarly, the bound forms of both chlorine and bromine in BCDMH were analyzed as free chlorine, in 
which the transformation from bromine to chlorine was on a molar basis (for example, 1 molar of BCDMH equals to 2 molars of $\mathrm{HClO}$ ).
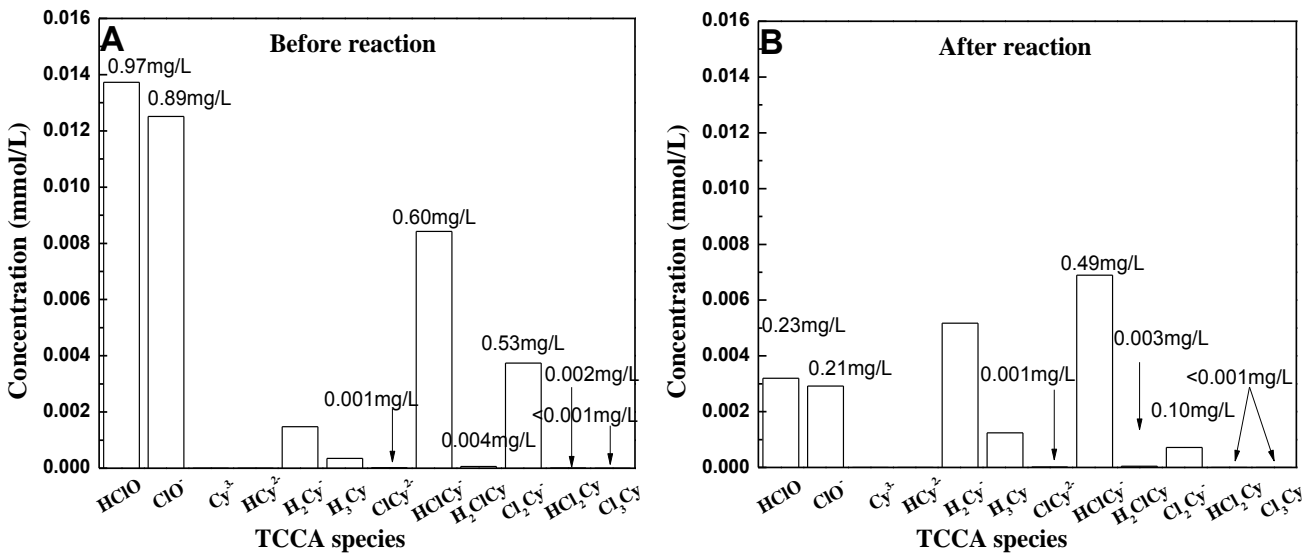

Figure 3.2 TCCA species before (A) and after (B) the reaction (filling water: tap (1.27 mg/L as TOC); temperature: $30{ }^{\circ} \mathrm{C}$; $\mathrm{pH}: 7.5$; reaction time: 24 hours; free chlorine: $3 \mathrm{mg} / \mathrm{L}$ as $\mathrm{Cl}_{2}$; BFA: $1 \mathrm{mg} / \mathrm{L}$ as TOC; disinfectants: TCCA)

Note: the values in figures indicate the concentrations of six chloroisocyanurate species as $\mathrm{Cl}_{2}$.

TOC and TN were determined by a TOC/TN high temperature combustion analyzer installed with an auto-sampler (multi N/C ${ }^{\circledR} 2100 \mathrm{~S}$, Analytikjena, Germany). The $\mathrm{pH}$ and temperature were measured by a pH meter (Accumet ${ }^{\circledR}$ Basic AB15) and a digital thermometer with an accuracy of $\pm 0.5{ }^{\circ} \mathrm{C}$ (PDQ400, USA) during sampling. The ions, including $\mathrm{F}^{-}, \mathrm{Cl}^{-}, \mathrm{NO}_{2}{ }^{-}, \mathrm{Br}^{-}, \mathrm{NO}_{3}{ }^{-}, \mathrm{PO}_{4}{ }^{3-}, \mathrm{SO}_{4}{ }^{2-}, \mathrm{Na}^{+}, \mathrm{NH}_{4}{ }^{+}, \mathrm{K}^{+}, \mathrm{Mg}^{2+}$, and $\mathrm{Ca}^{2+}$, were determined by an ion chromatography with an AG23 (4×50 mm) and an AS23 $(4 \times 250 \mathrm{~mm})$ as guard and analytical column for anions and a CG12A $(5 \mu \mathrm{m}, 3 \times 30$ $\mathrm{mm})$ and a CS12A (5 $\mu \mathrm{m}, 3 \times 150 \mathrm{~mm})$ as guard and analytical column for cations (IC, Dionex DX-120, Thermo Scientific, USA). 


\subsubsection{Field survey design}

A preliminary field survey in Singapore was conducted from March to May in 2013 in order to investigate the water quality under consideration of disinfection and formation of DBPs. The ambient and the pool water temperatures were around $28 \pm 3{ }^{\circ} \mathrm{C}$ all year round. The pool water quality may be different for different pools due to different pool operation conditions, e.g. the pool water recirculation ratio. Even for the same pool, the water condition might be different at the different time, e.g. the sampling time relative to the chlorine dosing time, and time-dependent solar radiation intensity. A total number of 37 samples were collected from 9 different swimming pools, while campus pool 1, representing a typical pool in Singapore, was chosen for intensive sampling to investigate the variation of pool water characteristics with time of the day. The 24 samples were collected from campus pool 1 during three periods, namely morning (08:30-09:30), noon (12:00-13:00), and evening (17:30-18:20). This allows us to obtain a further insight on how the solar UV strength affects the chlorine variation with time. The amount of the disinfectant, TOC and TN related to precursors for DBP formation, and the known DBPs including THMs and HAAs, were analyzed to get an overview of pool water quality in Singapore. The observations such as UV-induced chlorine variations, DBP accumulations, and recirculation of pool water, are also common to the pools in other places. 
3.2.3 Lab scale batch reactions under simulated swimming pool water conditions

The precursors of DBPs in the swimming pools mainly come from two sources, NOM from filling water and body fluids from swimmers. To simulate NOM, a tap water with a TOC of $1.27 \pm 0.13 \mathrm{mg} / \mathrm{L}$ and a $\mathrm{TN}$ of $2.15 \pm 0.16 \mathrm{mg} / \mathrm{L}$ was used. Nitrate-nitrogen accounted for $92.1 \%$ of the $\mathrm{TN}$ and the rest was mainly organic nitrogen (ammonia and nitrite were not detectable). The swimmer inputs were simulated by a BFA based on a recipe reported by Judd and Bullock (2003) as shown in Table 3.3. The BFA was the mixture of urine and sweat with a volume ratio of 1:4. The BFA stock solution with $\sim 5700 \mathrm{mg} / \mathrm{L}$ as TOC and $\sim 8700 \mathrm{mg} / \mathrm{L}$ as $\mathrm{TN}$ was prepared beforehand and diluted accordingly during the formation potential (FP) tests. The BFA concentration in pool water was reported to be roughly equal to 200 $\mathrm{ml}$ sweat and $50 \mathrm{ml}$ urine per cubic meter of pool water (Judd and Black, 2000). However the actual concentration relies on many variables, e.g. bather number, pool size and recirculation of water (Chowdhury et al., 2014). In our experiment, the BFA was adjusted to 1 or $5 \mathrm{mg} / \mathrm{L}$ as TOC. These TOC concentrations were consistent with the values in our preliminary survey (roughly in a single digital level $(\mathrm{mg} / \mathrm{L})$ as shown in Table 3.4) and in the studies by other researchers (Hansen et al., 2012a; Kanan and Karanfil, 2011). Amber glasses of $250 \mathrm{~mL}$ with PTFE seals were used as reactors to conduct DBP FP test. The use of amber glasses was to eliminate the effect of light exposure. Although the significance of our study originates from the advantage of chlorine stabilizer especially under sunlight conditions (Section 3.3.1), the direct sunlight exposure may bring some inevitable drawbacks to the experiments 
and becomes an interference factor. First, the sunlight intensity varies with times and days, thus may affect the experimental results from time to time. Second, natural sunlight has the potential to cause the decomposition of the formed THMs and HAAs (Chen et al., 2010). Reaction conditions were determined based on the local guidelines, and investigated or reported values for SPWs. Free chlorine/bromine as $\mathrm{Cl}_{2}$ was adjusted to 1 or $3 \mathrm{mg} / \mathrm{L}$ which is the lower or upper limit of Singapore swimming pool guideline (NEA, 2005). For $\mathrm{NaClO}$, the addition amount was based on the actual concentration of $\mathrm{NaClO}$ stock solution (max. $12 \%$ free chlorine, $\sim 10$ $40 \mathrm{~g} / \mathrm{L}$ as $\mathrm{Cl}_{2}$ ), measured before each FP test. For TCCA and BCDMH, it was determined based on theoretical calculation, assuming all the chlorine/bromine elements were transformed into $\mathrm{HClO}$ and/or $\mathrm{HBrO}$. TCCA and $\mathrm{BCDMH}$ in solid forms were freshly added before each experiment. Temperatures, namely 25, 30,35 and $40{ }^{\circ} \mathrm{C}$, were used in consideration of the temperature range of $26-40{ }^{\circ} \mathrm{C}$ in various types of pools, among which $30{ }^{\circ} \mathrm{C}$ was the normal water temperature in Singapore pools (ASHRAE, 2011). The $\mathrm{pH}$ was controlled at 7.50 based on the regulated range of 7.20-7.80, with a buffer solution with a mixture of $\mathrm{Na}_{2} \mathrm{HPO}_{4}$ and $\mathrm{NaH}_{2} \mathrm{PO}_{4}$ (8:2) with $\mathrm{PO}_{4}{ }^{3-}$ equal to $25 \mathrm{mM}$ (NEA, 2005). The reaction time was controlled at 24 hours for most FP experiments and extended to 138 hours for experiments to investigate the effect of reaction time and dosing methods on DBP formation. Detailed information of all experimental conditions is given in Table 3.5.

Table 3.3 Tap water characteristics and BFA components

\begin{tabular}{lccl|ll}
\hline & \multicolumn{3}{c}{ Tap water $^{\mathrm{a}}$} & \multicolumn{2}{c}{$\begin{array}{c}\mathrm{BFA}^{\mathrm{b}} \\
\mathrm{mg} / \mathrm{L}\end{array}$} \\
\hline TOC & $1.27 \pm 0.13(1.14-1.40)$ & $\mathrm{PO}_{4}{ }^{3-}$ & $<0.1$ & Urea & 14800 \\
TN & $2.15 \pm 0.16(1.98-2.29)$ & $\mathrm{SO}_{4}{ }^{2-}$ & $65.57 \pm 1.60(64.41-67.39)$ & Creatinine & 1800
\end{tabular}




\begin{tabular}{llll|ll}
$\mathrm{F}^{-}$ & $0.10 \pm 0.00(0.10-0.10)$ & $\mathrm{Na}^{+}$ & $9.66 \pm 0.94(8.60-10.39)$ & Uric acid & 490 \\
$\mathrm{Cl}^{-}$ & $15.73 \pm 1.33(14.22-16.68)$ & $\mathrm{NH}_{4}^{+}$ & $<0.01$ & Citric acid & 640 \\
$\mathrm{NO}_{2}^{-}$ & $<0.1$ & $\mathrm{~K}^{+}$ & $2.02 \pm 0.14(1.87-2.15)$ & L-Histidine & 1210 \\
$\mathrm{Br}^{-}$ & $<0.01$ & $\mathrm{Mg}^{2+}$ & $13.78 \pm 0.46(13.48-14.30)$ & Hippuric acid & 1710 \\
$\mathrm{NO}_{3}^{-}$ & $8.77 \pm 0.18(8.63-8.97)$ & $\mathrm{Ca}^{2+}$ & $76.93 \pm 2.49(74.59-79.55)$ & Ammonium chloride & 2000 \\
& & & & Sodium phosphate & 4300 \\
\hline
\end{tabular}

Note:

${ }^{\mathrm{a}}$ Mean \pm Standard Deviation (Minimum-Maximum), $\mathrm{n}=5$

${ }^{\mathrm{b}}$ BFA was proposed by Judd and Bullock (2003)

Table 3.4 Disinfectants, precursors and DBPs in different swimming pools in Singapore $^{\mathrm{a}}$

\begin{tabular}{|c|c|c|c|c|c|c|c|c|c|}
\hline & $\begin{array}{l}\text { Sample } \\
\text { No. }\end{array}$ & & Disinfectants & $\begin{array}{l}\text { Free } \\
\text { chlorine } \\
(\mathrm{mg} / \mathrm{L})\end{array}$ & $\begin{array}{l}\text { Combined } \\
\text { chlorine } \\
(\mathrm{mg} / \mathrm{L})\end{array}$ & $\begin{array}{l}\text { TOC } \\
(\mathrm{mg} / \mathrm{L})\end{array}$ & $\begin{array}{l}\mathrm{TN} \\
(\mathrm{mg} / \mathrm{L})\end{array}$ & $\begin{array}{l}\text { tTHMs } \\
(\mu \mathrm{g} / \mathrm{L})\end{array}$ & $\begin{array}{l}\text { tHAAs } \\
(\mu \mathrm{g} / \mathrm{L})\end{array}$ \\
\hline $\begin{array}{l}\text { Private } \\
\text { pools }\end{array}$ & 3 & Outdoor & N.A. & $\begin{array}{l}4.16 \pm 1.79 \\
(2.88- \\
6.21)\end{array}$ & $\begin{array}{l}0.28 \pm 0.27 \\
(0.03- \\
0.57)\end{array}$ & $\begin{array}{l}8.72 \pm 3.60 \\
(6.11- \\
12.83)\end{array}$ & $\begin{array}{l}6.63 \pm 3.09 \\
(4.76- \\
10.20)\end{array}$ & $\begin{array}{l}98 \pm 41 \\
(63- \\
142)\end{array}$ & $\begin{array}{l}620 \pm 491 \\
(343- \\
1187)\end{array}$ \\
\hline $\begin{array}{l}\text { Public } \\
\text { pools }\end{array}$ & 4 & Outdoor & N.A. & $\begin{array}{l}0.90 \pm 0.90 \\
(0.13- \\
1.88)\end{array}$ & $\begin{array}{l}0.11 \pm 0.09 \\
(0.03- \\
0.24)\end{array}$ & $\begin{array}{l}5.04 \pm 1.45 \\
(3.44- \\
6.54)\end{array}$ & $\begin{array}{l}6.31 \pm 2.53 \\
(2.94- \\
8.37)\end{array}$ & $\begin{array}{l}107 \pm 72 \\
(32- \\
170)\end{array}$ & $\begin{array}{l}817 \pm 771 \\
(191- \\
1906)\end{array}$ \\
\hline $\begin{array}{l}\text { Campus } \\
\text { pool } 1\end{array}$ & 24 & Outdoor & $\mathrm{NaClO}$ & $\begin{array}{l}0.57 \pm 0.75 \\
\text { (N.D.- } \\
\text { 2.86) }\end{array}$ & $\begin{array}{l}0.06 \pm 0.03 \\
\text { (N.D.- } \\
0.13 \text { ) }\end{array}$ & $\begin{array}{l}1.61 \pm 0.55 \\
(1.03- \\
3.64)\end{array}$ & $\begin{array}{l}4.33 \pm 0.19 \\
(4.05- \\
4.83)\end{array}$ & $\begin{array}{l}65 \pm 19 \\
(36- \\
118)\end{array}$ & $\begin{array}{l}1002 \pm 216 \\
(541- \\
1210)\end{array}$ \\
\hline $\begin{array}{l}\text { Campus } \\
\text { pool } 2\end{array}$ & 6 & Outdoor & $\mathrm{NaClO}$ & $\begin{array}{l}3.78 \pm 1.00 \\
(2.82- \\
5.40)\end{array}$ & $\begin{array}{l}0.20 \pm 0.09 \\
(0.10- \\
0.30)\end{array}$ & $\begin{array}{l}2.67 \pm 0.05 \\
(2.64- \\
2.70)\end{array}$ & N.A. & N.A. & 753 \\
\hline Average & -- & -- & -- & $2.35 \pm 1.71$ & $0.16 \pm 0.12$ & $4.51 \pm 2.55$ & $5.76 \pm 1.50$ & $90 \pm 37$ & $798 \pm 448$ \\
\hline
\end{tabular}

Notes: N.A.: not available; N.D.: not detectable.

${ }^{a}$ Mean \pm Standard Deviation (Minimum-Maximum), $n=3,4,24,6$

Table 3.5 An overview of all batch experimental conditions

\begin{tabular}{|c|c|c|}
\hline & Factors & Reaction conditions \\
\hline Section & Disinfectants: & Filling water: tap (1.27 mg/L as TOC); temperature: \\
\hline 3.3 .2 & $\begin{array}{l}\mathrm{NaClO} \text {, TCCA and } \\
\text { BCDMH. }\end{array}$ & $\begin{array}{l}30{ }^{\circ} \mathrm{C} \text {; } \mathrm{pH}: 7.5 \text {; reaction time: } 24 \text { hours; free } \\
\text { chlorine/bromine: } 3 \mathrm{mg} / \mathrm{L} \text { as } \mathrm{Cl}_{2} \text {; BFA: } 1 \mathrm{mg} / \mathrm{L} \text { as } \\
\text { TOC. }\end{array}$ \\
\hline Section & Dosing methods: & Filling water: $\operatorname{tap}(1.40 \mathrm{mg} / \mathrm{L}$ as TOC $)$; temperature: \\
\hline 3.3 .3 & $\begin{array}{l}\text { continuous and shock } \\
\text { dosing. }\end{array}$ & $\begin{array}{l}30{ }^{\circ} \mathrm{C} ; \mathrm{pH}: 7.5 \text {; reaction time: } 138 \text { hours; BFA: } 1 \\
\mathrm{mg} / \mathrm{L} \text { as } \quad \text { TOC; } \\
\mathrm{NaClO} / \mathrm{TCCA} / \mathrm{BCDMH} \text {. }\end{array}$ \\
\hline $\begin{array}{l}\text { Section } \\
3.3 .4\end{array}$ & $\begin{array}{l}\text { Temperatures: } 25,30 \text {, } \\
35 \text { and } 40^{\circ} \mathrm{C} \text {. }\end{array}$ & $\begin{array}{l}\text { Filling water: tap }(1.14 \mathrm{mg} / \mathrm{L} \text { as } \mathrm{TOC}) \text {; } \mathrm{pH}: 7.5 \text {; } \\
\text { reaction time: } 24 \text { hours; free chlorine/bromine: } 3 \\
\mathrm{mg} / \mathrm{L} \text { as } \mathrm{Cl}_{2} ; \mathrm{BFA}: 1 \mathrm{mg} / \mathrm{L} \text { as TOC; disinfectants: } \\
\mathrm{NaClO} / \mathrm{TCCA} / \mathrm{BCDMH}\end{array}$ \\
\hline Section & BFA and free & Filling water: tap (1.27 mg/L as TOC); temperature: \\
\hline 3.3 .5 & $\begin{array}{l}\text { chlorine/bromine: } \\
\mathrm{BFA} \text { of } 0,1 \text { and } 5 \\
\mathrm{mg} / \mathrm{L} \text { and } \\
\text { chlorine/bromine of } 1 \\
\text { and } 3 \mathrm{mg} / \mathrm{L} \text { as } \mathrm{Cl}_{2} \text {. }\end{array}$ & $\begin{array}{l}30{ }^{\circ} \mathrm{C} ; \mathrm{pH}: \quad 7.5 \text {; reaction time: } 24 \text { hours; } \\
\text { disinfectants: NaClO/TCCA/BCDMH. }\end{array}$ \\
\hline Section & Matrix: NOM and & Temperature: $30{ }^{\circ} \mathrm{C} ; \mathrm{pH}: 7.5$, reaction time: 24 \\
\hline
\end{tabular}




\begin{tabular}{ll}
\hline 3.3 .6 & hours; free chlorine/bromine: $3 \mathrm{mg} / \mathrm{L}$ as $\mathrm{Cl}_{2} ; \mathrm{NOM}$ \\
& or $\mathrm{BFA}: 1 \mathrm{mg} / \mathrm{L}$ as $\mathrm{TOC} ;$ disinfectants: \\
& NaClO/TCCA/BCDMH (NOM vs. BFA). \\
& Filling water: $\mathrm{DI}$; temperature: $30{ }^{\circ} \mathrm{C} ; \mathrm{pH}: 7.5 ;$ \\
& reaction time: 24 hours; free chlorine: $3 \mathrm{mg} / \mathrm{L}$ as \\
& $\mathrm{Cl}_{2}$; ammonium chloride as $\mathrm{TN}$ and other individual \\
& components as TOC: $1 \mathrm{mg} / \mathrm{L} ;$ disinfectants: $\mathrm{NaClO}$ \\
& (individual components in BFA) \\
\hline
\end{tabular}

Note:

The experiments were conducted in duplicate.

\subsubsection{Two-stage models for chlorine decay and DBP formation}

Second order kinetic models were used to simulate both chlorine decay and THM/HAA formation (Eqs. (3.1) and (3.2)). The significantly different reaction rates under different concentrations made the reactions behave like two-stage processes (stage 1 with the fast reaction when $0 \leq t \leq 6 \mathrm{~h}$ and stage 2 with the slow reaction when $6 \mathrm{~h}<t \leq 138 \mathrm{~h}$, see Section 3.3.3).

$$
\begin{gathered}
\frac{1}{C t}-\frac{1}{C o}=k_{1} t \\
\frac{d[T H M \text { or } H A A]}{d t}=k_{2}[T O C]\left[C l_{2}\right] \approx k_{2} A\left[C l_{2}\right]
\end{gathered}
$$

where $k_{1}$ and $k_{2}$ are the rate constants for the second order chlorine decay, and THM or HAA formation $\left(\mathrm{L} / \mathrm{mg} \cdot \mathrm{h}^{-1}\right)$, respectively, $C_{o}$ and $C_{t}$ are the free chlorine concentrations at 0 and $t \mathrm{~h}(\mathrm{mg} / \mathrm{L}), t$ is the reaction time (h), $A$ is the initial TOC concentration from NOM and BFA $(\mathrm{mg} / \mathrm{L})$. There was an assumption that TOC reduction was negligible in this model. The constant $k_{l}$ was derived from variables $C_{t}$ and $t$ with the linear least square best-fit method. 
By substituting Eq. (3.1) into Eq. (3.2), Eq. (3.3) was obtained and further integrated to generate Eq. (3.4),

$$
\begin{gathered}
\frac{d[T H M \text { or } H A A]}{d t}=\frac{k_{2} A}{k_{1} t+1 / C o} \\
{[T H M \text { or } H A A]=\frac{k_{2} A}{k_{1}} \ln \left|\frac{1}{C o}+k_{1} t\right|+B}
\end{gathered}
$$

where $B$ is an integration constant $(\mathrm{mg} / \mathrm{L})$. The constants $k_{2}$ and $B$ were calculated by the best-fit method (least squares) with variables of [THM or HAA] and $t$.

\subsection{Results and discussion}

\subsubsection{Disinfectant, precursors and DBP occurrence in Singapore pools}

The average free chlorine concentrations in private pools and one campus pool were 4.2 and $3.8 \mathrm{mg} / \mathrm{L}$ which were higher than the regulated range between 1 to $3 \mathrm{mg} / \mathrm{L}$ (NEA, 2005). However, in public pools and another campus pool the concentrations were lower than $1 \mathrm{mg} / \mathrm{L}$ or even undetectable (Table 3.4). The chlorine concentrations were significantly different in different pools. In order to further understand the chlorine temporal distribution, the results of a more comprehensive investigation in campus pool 1 are shown in Figure 3.3. It illustrates the effect of sampling time on the chlorine concentration. The chlorine was relatively high in the morning (before 9:30 am), low and even undetectable at noon (around 12:00) and slightly increased during evening period (around 17:30). The varied bather load may (and very likely) affect the chlorine concentration in pools (Afifi and Blatchley Iii, 2015). However, the number of swimmers remained reasonably consistent from 
morning till early afternoon and increased significantly in the evening. In addition, the TOC values did not show a systematic trend with time. Therefore, the impact of bather loads on chlorine demand can be negligible. The change of the UV irradiation characterized by UV index showed quite opposite behavior than the chlorine concentration. The UV index depending on both UVA and UVB radiation ranges from 0 to $11+$ and indicates the potential for harmful effects to the human skin and eyes. The curve in Figure 3.3 was adapted from a typical daily variation of UV index in Singapore reported by NEA. The $\mathrm{ClO}^{-}$with an absorption maximum of $290 \mathrm{~nm}$ undergoes photolysis under UV exposure (Wojtowicz, 1996). Thus, low chlorine at noon period might result from the extremely high UV radiation level. The risk for bacterial growth and infection diseases to swimmers may increase when the free chlorine is too low. On the other hand, the formation of DBPs will be increased when the free chlorine is too high. Carbonaceous and nitrogenous compounds are precursors of DBPs quantified by TOC and TN. In private and public pools, TOC and TN were much higher than those in campus pools. The average TOC in all investigated pools was $2.81 \pm 2.55 \mathrm{mg} / \mathrm{L}$ which was similar to $3.5 \pm 1.6 \mathrm{mg} / \mathrm{L}$ obtained from 50 pool samples by De Laat et al. (2011). 


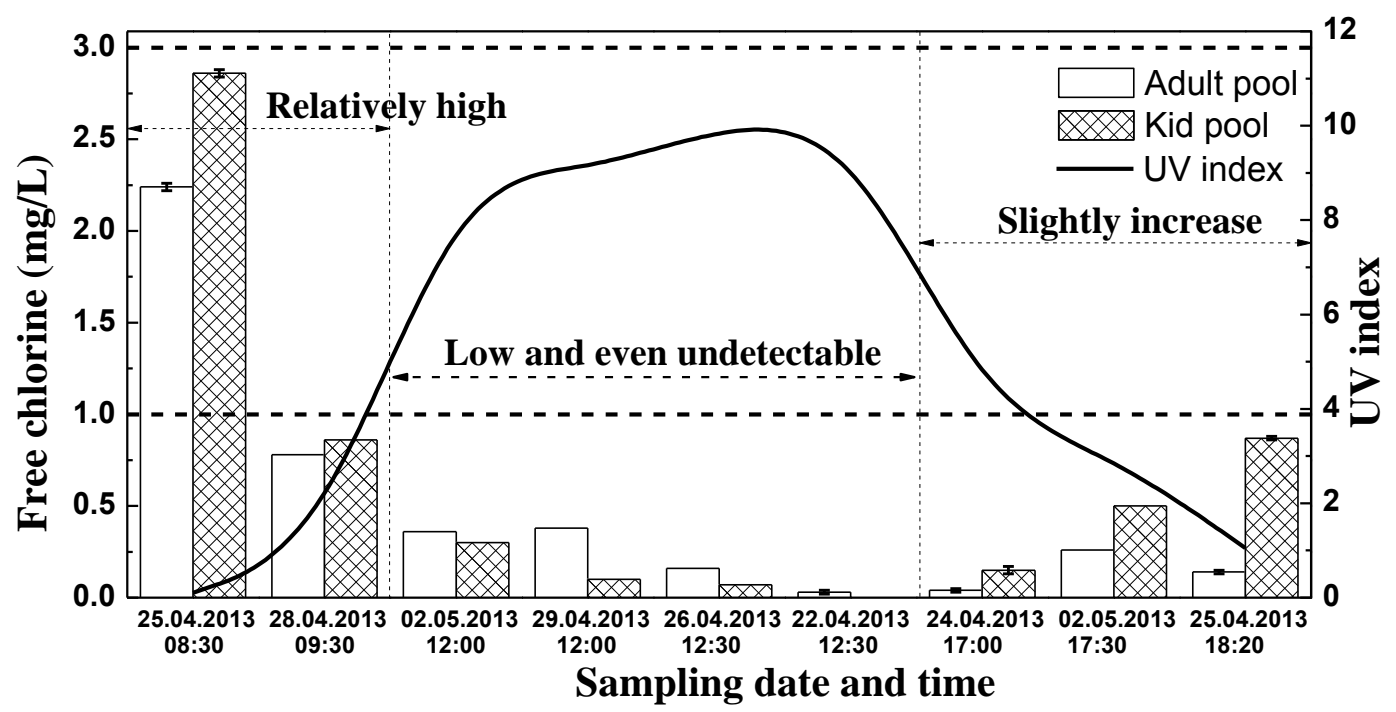

Figure 3.3 Free chlorine concentrations at different sampling dates and times in campus pool 1 (empty and shaded columns) are compared to the typical daily variation of the UV index in Singapore (solid line; adapted from NEA at http://www.nea.gov.sg/training-knowledge/weather-climate/uvradiation-uvindex). The horizontal dashed lines represent the range of free chlorine regulated by NEA Singapore.

DBP concentrations, especially HAAs, in the investigated pools were much higher than EPA regulation would allow (THM $\leq 80 \mu \mathrm{g} / \mathrm{L}$ and HAA $\leq 60 \mu \mathrm{g} / \mathrm{L}$ ) for DWs (EPA, 1998). Chloroform, di- and trichloroacetic acid were the main contributors of THMs (80-90\%) and HAAs (96-97\%) due to the low bromide levels detected in pool water. The average di- and trichloroacetic acid concentrations in a campus outdoor pool for adults were 462 and $492 \mu \mathrm{g} / \mathrm{L}$, respectively and the average concentration of total haloacetic acids (tHAAs) in all investigated pools was $798 \mu \mathrm{g} / \mathrm{L}$ with a standard deviation of $448 \mu \mathrm{g} / \mathrm{L}$. Similar tHAAs were reported as $826 \mu \mathrm{g} / \mathrm{L}$ for 39 outdoor pools in Canada (Simard et al., 2013). Wang et al. (2014) have reported much higher individual di- and trichloroacetic acid concentrations as 700 and 680 $\mu \mathrm{g} / \mathrm{L}$, respectively, for 6 outdoor pools in the U.S. However, much lower values of 132 and $249 \mu \mathrm{g} / \mathrm{L}$ for outdoor pools in Germany were reported (Stottmeister and 
Naglitsch, 1996). The similar/different results among these four studies may be mainly due to the similar/different disinfection conditions for pools. For example, 1$3 \mathrm{mg} / \mathrm{L}$ and $0.8-3 \mathrm{mg} / \mathrm{L}$ are the regulated free chlorine concentration ranges for outdoor pools in Singapore and Canada, respectively, and 1-5 mg/L for U.S. pools, and only 0.3-0.6 mg/L for German pools (Canada, 2016; Germany, 2012; NEA, 2005; NSPF, 2006). High free chlorine concentrations are directly linked to high DBP formation although further parameters will contribute as well. Therefore, the effect of free chlorine concentration on DBP formation will be further discussed in Sections

\subsection{3 and 3.3.5.}

The challenges revealed by the preliminary survey are as follows: 1) For typical outdoor pools in Singapore, high chlorine decomposition by UV radiation from sunlight has to be considered; 2) High THM and HAA concentrations in investigated swimming pools have to be considered. In addition, a mandatory DBP regulation of swimming pools in Singapore is still not available. It is a pressing mission to find an ideal solution to overcome these challenges.

\subsubsection{Comparison of different disinfectants}

The comparison of three different disinfectants was evaluated taking into account the residual free chlorine/bromine concentrations and the DBP formation. The conditions were as follows: filling water: tap; temperature: $30^{\circ} \mathrm{C} ; \mathrm{pH}: 7.5$; reaction time: 24 hours; free chlorine/bromine: $3 \mathrm{mg} / \mathrm{L}$ as $\mathrm{Cl}_{2}$; BFA: $1 \mathrm{mg} / \mathrm{L}$ as TOC. 
Figure 3.4 reveals that after 24 hours' reaction time much higher residual free chlorine/bromine concentration in the simulated pool water disinfected by TCCA was corresponding to the lower DBP formation (reduction of 16\%) compared to $\mathrm{NaClO}$. The stabilization effect of TCCA resulted in a slower release of free chlorine, causing less chlorine available for DBP formation and thus higher residual free chlorine. The bound forms of chlorine, mainly including $\mathrm{HClCy}^{-}$and $\mathrm{Cl}_{2} \mathrm{Cy}^{-}$, occupied more than half of the residual free chlorine, which offer potential $\mathrm{HClO} / \mathrm{ClO}^{-}$release (Figure 3.2). In addition, frequent $\mathrm{NaClO}$ addition to maintain a high-enough free chlorine concentration also leads to more DBP formation. Thus, TCCA can guarantee less DBP formation than $\mathrm{NaClO}$ on the basis of same initial chlorine dose. Meanwhile, some studies show that an excess of cyanuric acid may delay the inactivation of microbes such as streptococcus faecalis and cryptosporidium parvum (Murphy et al., 2015; Wojtowicz, 1996). However, based on the empirically fitted regression equation $t_{0.99}=0.0268 *[\mathrm{HClO}]^{-1.014}$ proposed by Wojtowicz (1996), linking between 99\% kill time and $\mathrm{HClO}$ concentration, the estimated kill time of enteric bacteria by TCCA could only be delayed by $0.011 \mathrm{~min}$ compared to $\mathrm{NaClO}$. Nevertheless, how to control the relative total cyanurate and chlorine in pools to obtain joint benefits between sufficient bacterial killing and DBP minimization would be our future interests. Residual free bromine of BCDMH was similar to residual free chlorine of $\mathrm{NaClO}$. Therefore, the stabilization effect of bromine was not observed for BCDMH. The compound patterns of THMs and HAAs were mainly composed of bromoform, di- and tribromoacetic acid for BCDMH and of chloroform, di- and trichloroacetic acid for $\mathrm{NaClO}$ and TCCA (refer to Table 3.6 
and Table 3.7). BCDMH also produced more mixed chloro-bromo species, e.g. $\mathrm{CHBr}_{2} \mathrm{Cl}$, than the other two disinfectants. The DBPs generated from BCDMH were about two-time higher compared to those from $\mathrm{NaClO}$ and TCCA (calculated as total mass). The normalized THM concentrations (calculated as chloroform) were still higher for $\mathrm{BCDMH}$ than those for $\mathrm{NaClO}$ and TCCA with an increase by $17 \%$ and 53\% (Table 3.6). This indicates that $\mathrm{BCDMH}$ and its corresponding bromine species are more reactive than other disinfectants and their corresponding chlorine species. A more detailed elaboration will be presented in the following Section 3.3.3.
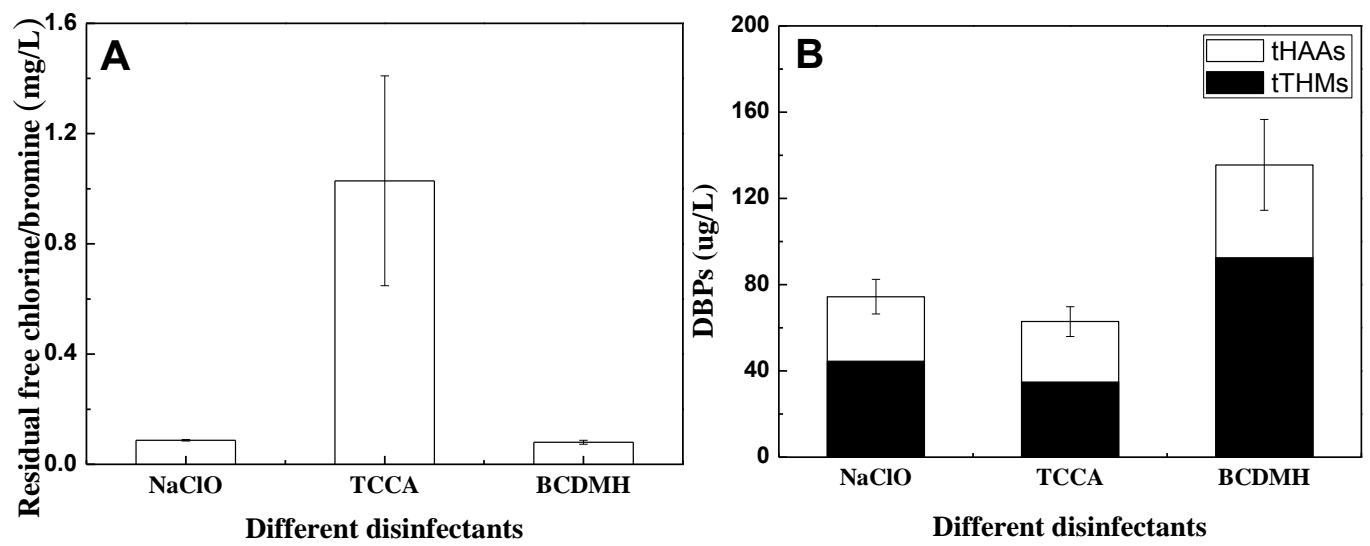

Figure 3.4 Comparison of three different disinfectants for residual free chlorine/bromine and DBP formation (filling water: tap $(1.27 \mathrm{mg} / \mathrm{L}$ as TOC); temperature: $30{ }^{\circ} \mathrm{C}$; $\mathrm{pH}$ : 7.5 ; reaction time: 24 hours; free chlorine/bromine: $3 \mathrm{mg} / \mathrm{L}$ as $\mathrm{Cl}_{2}$; BFA: $1 \mathrm{mg} / \mathrm{L}$ as TOC; disinfectants: $\mathrm{NaClO} / \mathrm{TCCA} / \mathrm{BCDMH}$ )

Table 3.6 Formation of THMs by three disinfectants (filling water: tap $(1.27 \mathrm{mg} / \mathrm{L}$ as TOC); temperature: $30{ }^{\circ} \mathrm{C}$; $\mathrm{pH}: 7.5$; reaction time: 24 hours; free chlorine/bromine: $3 \mathrm{mg} / \mathrm{L}$ as $\mathrm{Cl}_{2}$; BFA: $1 \mathrm{mg} / \mathrm{L}$ as TOC; disinfectants: $\mathrm{NaClO} / \mathrm{TCCA} / \mathrm{BCDMH}$ )

\begin{tabular}{lllllrl}
\hline & $\mathrm{CHCl}_{3}$ & $\mathrm{CHBrCl}_{2}$ & $\mathrm{CHBr}_{2} \mathrm{Cl}$ & $\begin{array}{l}\mathrm{CHBr}_{3} \\
\mu \mathrm{tTHMs}\end{array}$ & $\begin{array}{l}\text { tTHMs as } \\
\mathrm{CHCl}_{3}\end{array}$ \\
\hline $\mathrm{NaClO}$ & $34.6 \pm 3.2$ & $6.5 \pm 0.5$ & $2.7 \pm 0.2$ & $0.6 \pm 0.1$ & $44.4 \pm 3.9$ & $41.2 \pm 3.7$ \\
$\mathrm{TCCA}$ & $24.7 \pm 3.5$ & $6.4 \pm 0.2$ & $3.0 \pm 0.1$ & $0.6 \pm 0.1$ & $34.8 \pm 3.9$ & $31.4 \pm 3.7$ \\
$\mathrm{BCDMH}$ & $3.9 \pm 0.5$ & $5.5 \pm 0.6$ & $9.6 \pm 0.7$ & $73.5 \pm 12.4$ & $92.5 \pm 14.2$ & $48.1 \pm 7.2$ \\
\hline
\end{tabular}

Note:

Standard deviation was calculated out at least from duplicate samples 
Table 3.7 Formation of HAAs by three disinfectants (filling water: tap $(1.27 \mathrm{mg} / \mathrm{L}$ as TOC); temperature: $30^{\circ} \mathrm{C}$; $\mathrm{pH}$ : 7.5 ; reaction time: 24 hours; free chlorine/bromine: $3 \mathrm{mg} / \mathrm{L}$ as $\mathrm{Cl}_{2}$; BFA: $1 \mathrm{mg} / \mathrm{L}$ as TOC; disinfectants: $\mathrm{NaClO} / \mathrm{TCCA} / \mathrm{BCDMH}$ )

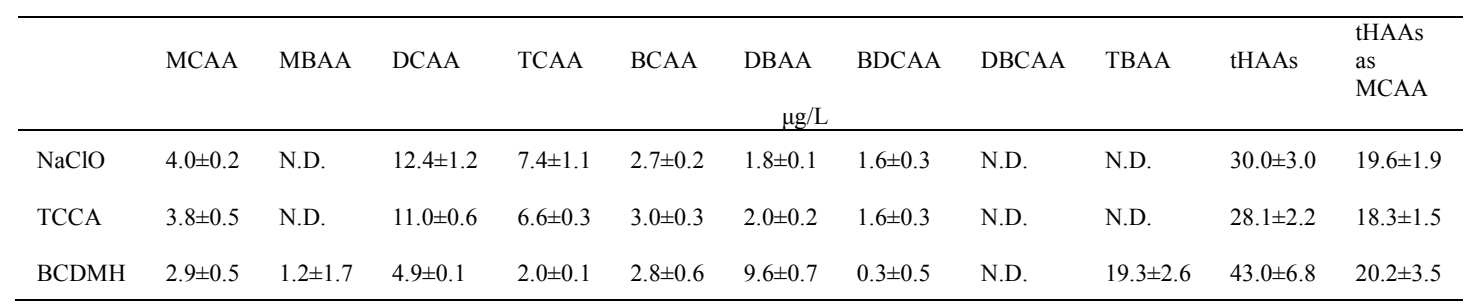

Note:

N.D.: not detectable.

Standard deviation was calculated out at least from duplicate samples.

\subsubsection{Effect of reaction time and dosing methods on DBP formation}

The long retention time of organic matters due to the pool water recirculation, and the shock or continuous addition of disinfectants, lead to a continuous formation of DBPs. The effect of reaction time on DBP formation was investigated by two disinfectant dosing methods: 1) continuous dosing; 2) shock dosing. The shock dosing was done by one addition of $15 \mathrm{mg} / \mathrm{L} \mathrm{Cl}_{2}$ at the beginning of the experiment. The continuous dosing was done by addition of $3 \mathrm{mg} / \mathrm{L}$ periodically at seven successive time points $(0,6,18,42,66,90,114$ hours). The additions at 90 and 114 hours aimed to further investigate the effect of total chlorine/bromine addition on DBP formation. The comparison of the dosing methods aims to explore whether it is meaningful for the pool water disinfection practice to use the results of shock chlorination like it is widely done for the determination of formation potential of the DBPs (Hansen et al., 2012a; Kanan and Karanfil, 2011). The experimental conditions were: filling water: tap; temperature: $30^{\circ} \mathrm{C} ; \mathrm{pH}$ : 7.5; reaction time: 138 hours; BFA: $1 \mathrm{mg} / \mathrm{L}$ as TOC; disinfectants: NaClO/TCCA/BCDMH. 

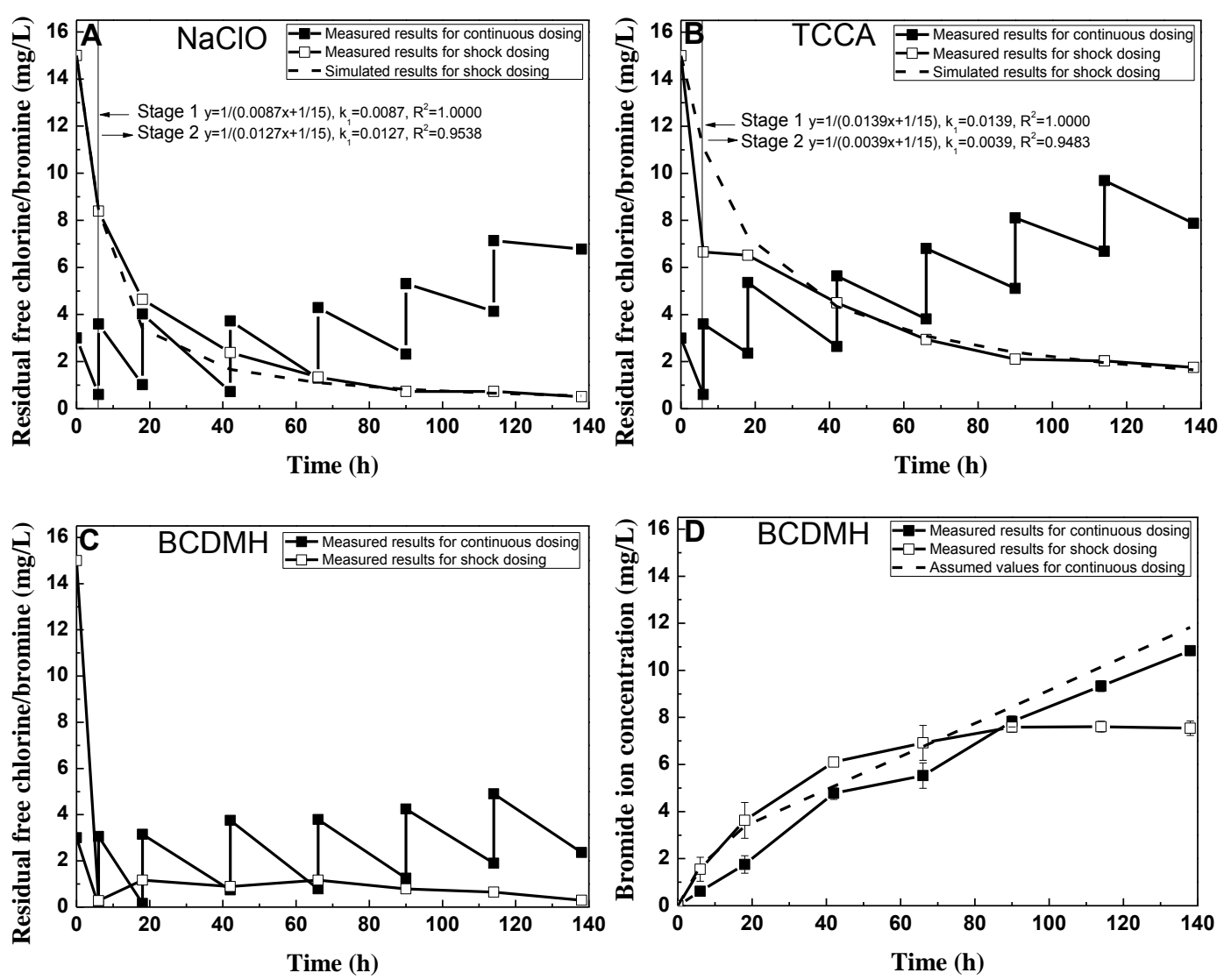

Figure 3.5 Effect of reaction time and dosing methods on residual free chlorine/bromine, formation of bromide ions, and two-stage chlorine decay models (filling water: tap $\left(1.40 \mathrm{mg} / \mathrm{L}\right.$ as TOC); temperature: $30^{\circ} \mathrm{C}$; $\mathrm{pH}$ : 7.5 ; reaction time: 138 hours; BFA: $1 \mathrm{mg} / \mathrm{L}$ as TOC; disinfectants: $\mathrm{NaClO} / \mathrm{TCCA} / \mathrm{BCDMH}$ )

Figure 3.5 reveals the effect of the reaction time and the dosing methods on residual free chlorine/bromine concentration during 138 hours. For $\mathrm{NaClO}$ and TCCA, the overall trend was a gradual decrease of residual free chlorine concentration for the shock experiments and a slow increase for the continuous experiments. Comparing the same total chlorine dosage at 90 hours $(3 \times 5 \mathrm{mg} / \mathrm{L}$ vs. $15 \mathrm{mg} / \mathrm{L})$, the residual free chlorine concentrations of $\mathrm{NaClO}$ and TCCA for the continuous experiments were slightly higher than those for shock experiments. The relatively lower residual chlorine concentration in shock experiments could be explained by higher chlorine 
consumption caused by the oxidation reaction at higher chlorine starting concentrations (15 mg/L). Acero et al. (2005) also reported that the reaction rate constant increased five times when chlorine dosing concentrations increased from $3 \mathrm{mg} / \mathrm{L}$ to $\sim 15 \mathrm{mg} / \mathrm{L}$. After 90 hours, the further excess of disinfectants in the continuous experiments made a significant difference in residual chlorine compared to the shock experiments (ANOVA, $F$-distribution, $P \leq 0.05$ ). Similarly, Hansen et al. (2013) found the final chlorine concentration of shock dosing after 48 hours was less but not so significant than that of continuous dosing $(3 \times 3.33 \mathrm{mg} / \mathrm{L} \mathrm{vs} .10 \mathrm{mg} / \mathrm{L})$. For TCCA, the final chlorine concentration in the shock experiment was $1.76 \mathrm{mg} / \mathrm{L}$, which was much higher than $0.51 \mathrm{mg} / \mathrm{L}$ for $\mathrm{NaClO}$, indicating the stabilization effect of TCCA for the free chlorine concentrations.

For $\mathrm{BCDMH}$, the residual disinfectant concentrations at each measuring point were quite low (less than $2 \mathrm{mg} / \mathrm{L}$ in most tests). Figure 3.5D shows that the measured bromide ion concentrations almost resembled the calculated values assumed that the bromine atoms in $\mathrm{BCDMH}$ are quantitatively transferred to bromide ions for the continuous experiment. A preliminary experiment revealed that the dissolution of $25.5 \mathrm{mg} \mathrm{BCDMH}$ in $1 \mathrm{~L}$ water (equivalent to $15 \mathrm{mg} / \mathrm{L} \mathrm{Cl}_{2}$ ) took about 26 hours, which indicates a rather slow reaction of $\mathrm{BCDMH}$ in water. However, after dissolution, bromine in $\mathrm{BCDMH}$ is released as $\mathrm{HBrO}$ with a high oxidation activity. For the hydrolyzed product $\mathrm{HClO}$, it can rapidly react with bromide ions to form $\mathrm{HBrO}$ (Watson et al., 2015). Most of the $\mathrm{HBrO}$ is rapidly reduced to bromide ions (mg/L level as shown in Figure 3.5D) and part is reacted with organic matter and 
transformed to Br-DBPs $(\mu \mathrm{g} / \mathrm{L}$ level as shown in Figure 3.6C) (Zhang and Matson, 1989). Bromide ions have been reported to promote the formation of both THMs and HAAs by bromine substitution of Cl-DBPs or further halogenations of organic matter (Hua et al., 2006). Therefore, slow dissolution of BCDMH and fast $\mathrm{HBrO}$ consumption could be the explanation of low free bromine residuals.
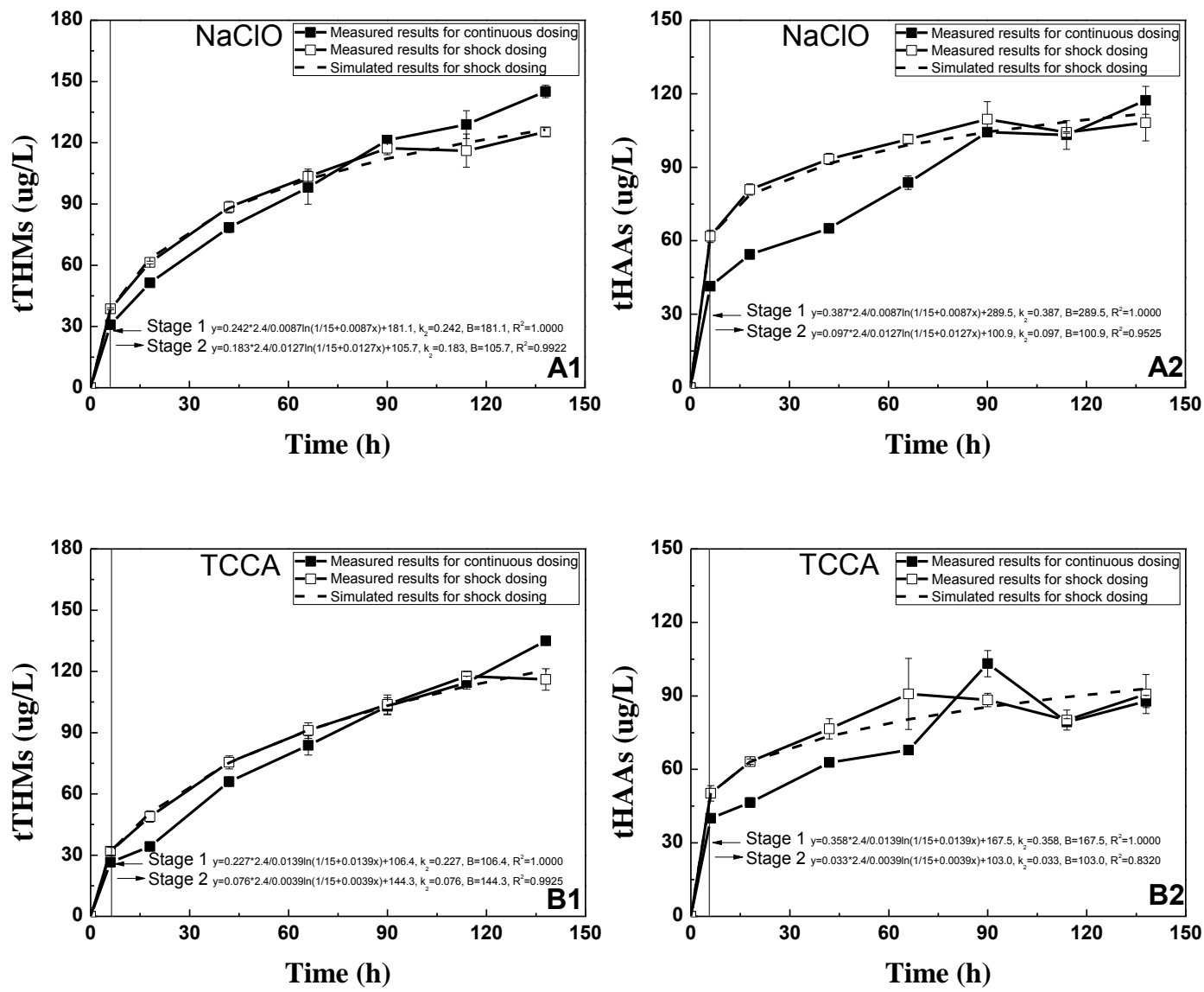

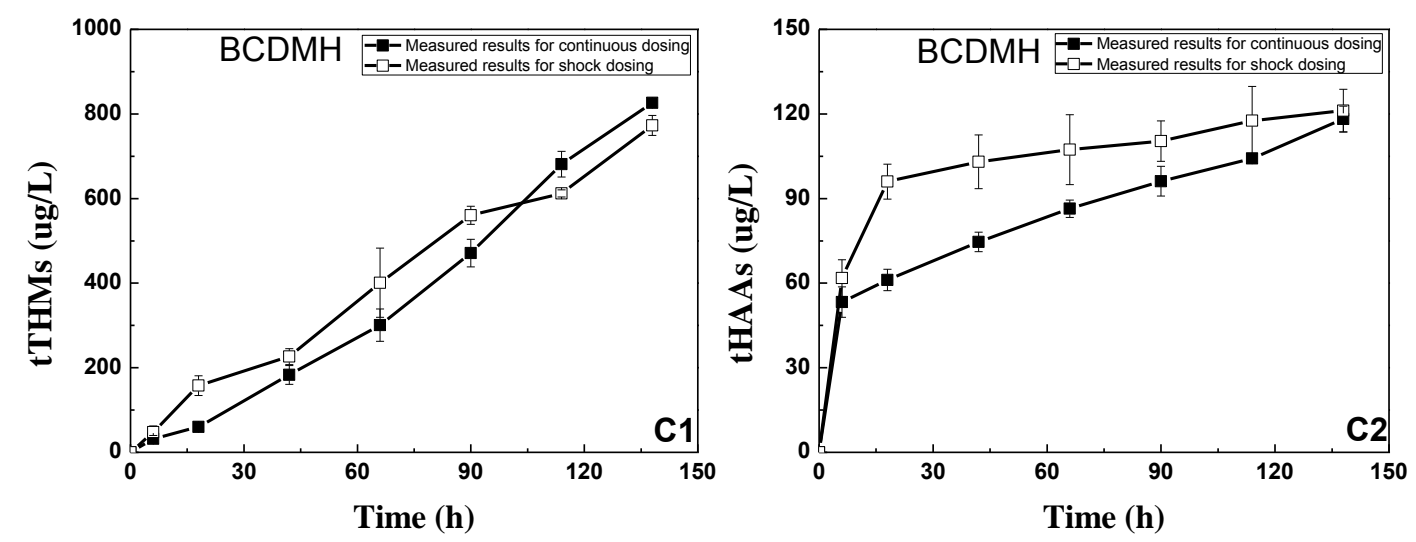

Figure 3.6 Effect of reaction time and dosing methods on THM and HAA formation, and two-stage DBP formation models (filling water: tap $(1.40 \mathrm{mg} / \mathrm{L}$ as TOC); temperature: $30{ }^{\circ} \mathrm{C}$; $\mathrm{pH}$ : 7.5 ; reaction time: 138 hours; BFA: $1 \mathrm{mg} / \mathrm{L}$ as TOC; disinfectants: $\mathrm{NaClO} / \mathrm{TCCA} / \mathrm{BCDMH}$ )

Figure 3.6 shows the relationship between THM and HAA formation and reaction time using two dosing methods. THM concentrations increased gradually with the increase of reaction time no matter which disinfectants and dosing methods were used. The whole reaction can be divided into two stages where 90 hours was a critical point. Before 90 hours, THMs increased slightly but not much faster in the shock experiments than those in the continuous experiments. After 90 hours, the opposite trend was discovered. For BCDMH, a continuous increase of THMs was observed until the end ( 138 hours) of the shock experiment. The slower reaction of BCDMH compared to that of $\mathrm{NaClO}$ and TCCA may result from the slower dissolution of BCDMH (Figure 3.6C1). However, the remarkableness is the high levels of THMs for BCDMH $(\sim 800 \mu \mathrm{g} / \mathrm{L})$ compared to $\mathrm{NaClO}$ and TCCA $(\sim 120 \mu \mathrm{g} / \mathrm{L})$ in the end of the reactions. This is mainly because bromide ion can be oxidized into $\mathrm{HBrO}$ in the presence of $\mathrm{HClO}$ and promotes the DBP formation (Hua et al., 2006). In addition, the Br-THMs have higher molecular weight than the chlorinated ones. The 
production of HAAs for the first 6 hours was remarkable with $51.0-57.2 \%$ of final HAA formation (at 138 hours) in the shock dosing experiments. The HAA formation in continuous experiments was much slower, but the difference became smaller when it approached to 90 hours. Interestingly, different from THM cases, HAA formation from BCDMH was similar, or slightly higher, compared to those from TCCA and $\mathrm{NaClO}$. This is possibly due to the limited amount of BFA which is found to be the main precursors of HAAs. More details will be provided later in Section 3.3.6. Hua et al. also observed similarly that the increase of HAAs in the presence of bromine was less than that of THMs. Results show that both residual free chlorine/bromine and THM/HAA formation depend mainly on the total chlorine/bromine dosage instead of the dosing methods if the reaction time is sufficient. A similar phenomenon has been observed by Hansen et al. (2013). Thus, a shock test in the laboratory is acceptable for selected studies of DBP formation. In addition, the lower formation of DBPs (reduction by 10-15\%, Figure 3.6) and higher residual chlorine (increase by 1.4-2.8 mg/L, Figure 3.5) for TCCA at 90 hours compared to $\mathrm{NaClO}$ is consistent with our previous finding (Section 3.3.2), which further validates the stability effect of TCCA to chlorine.

Two-stage kinetic based models were derived from the initial fast chlorine decay corresponding to the fast THM and HAA formation during the first several hours and subsequent slow reactions lasting from hours to days. The fast chlorine decay can be attributed to the additional high oxidation process apart from chlorination at initial high concentration stage. The fast THM and HAA formation could be the main 
contribution of the active part of organic matters (Sohn et al., 2004). The second order kinetic model for THM and HAA formation comes from the hypothetical reaction equation: $\mathrm{Cl}_{2}+\mathrm{TOC} \rightarrow \mathrm{THM} / \mathrm{HAA}$ which is widely used in other similar studies (Li et al., 2012; Sohn et al., 2004). The second order model for chlorine decay was chosen due to its better fitting compared to first order model. The critical point was set at 6 hours. Thus, stage 1 was from 0 to 6 hours and stage 2 was from 6 to 138 hours (Figure 3.5 and Figure 3.6). A was equal to the initial TOC concentration $2.4 \mathrm{mg} / \mathrm{L}$ (refer to Section 3.2.4). Good correlation coefficients $R^{2}$ at 0.9538 and 0.9483 were achieved for the chlorine decay with $\mathrm{NaClO}$ and TCCA, respectively. The corresponding correlation coefficients for the DBP formation were excellent, especially for the formation of THMs, $R^{2}=0.9922,0.9925$ for $\mathrm{NaClO}$ and TCCA, respectively.

This kinetic-based two-stage decay and formation model was built based on batch experiments under conditions consistent to the real pool environments (e.g. temperature: $30{ }^{\circ} \mathrm{C}, \mathrm{pH}: 7.5$, TOC: $\left.2.4 \mathrm{mg} / \mathrm{L}\right)$. Although the continuous addition of disinfectants is more ideal compared with the shock dosing, both dosing methods result in similar residual chlorine and DBP formation (Section 3.3.3). However, due to the complicated water matrix in real pools, this model approach can be further improved by correlating the reaction rate constants $\left(k_{l}\right.$ and $\left.k_{2}\right)$ with other factors (e.g. TOC, temperature, $\mathrm{pH}$, etc.). This model carries the potentials for the pool managers to estimate a suitable chlorine concentration and the resulting DBP formation for an optimized operation. The inputs needed to feed the model are the initial free chlorine 
and TOC concentrations, from which disinfectant consumption and DBP formation over time can be deduced.

\subsubsection{Effect of temperature on DBP formation}

Temperature control is generally determined by the purpose of pools. For example, lower temperatures $\left(26-28{ }^{\circ} \mathrm{C}\right)$ are used for competitive pools and higher temperatures $\left(30-40^{\circ} \mathrm{C}\right)$ are for spa pools. The temperature effect on DBP formation has been reported in another study (Kanan and Karanfil, 2011). However, the investigation of temperature dependence of DBP formation by TCCA and BCDMH was not as much as that by $\mathrm{NaClO}$. Four different temperatures, including 25, 30, 35 and $40{ }^{\circ} \mathrm{C}$, were used in this work. The reaction conditions were: filling water: tap; pH: 7.5; reaction time: 24 hours; free chlorine/bromine: $3 \mathrm{mg} / \mathrm{L}$ as $\mathrm{Cl}_{2}$; BFA: $1 \mathrm{mg} / \mathrm{L}$ as TOC; disinfectants: $\mathrm{NaClO} / \mathrm{TCCA} / \mathrm{BCDMH}$.

Figure 3.7 illustrates that THM concentrations increased rapidly with the incremental increase of temperature. For $\mathrm{NaClO}$ and $\mathrm{BCDMH}$ at $25^{\circ} \mathrm{C}$, THMs were 31.0 \pm 1.1 and $38.3 \pm 2.7 \mu \mathrm{g} / \mathrm{L}$, respectively. THM concentrations almost doubled when the temperature was increased to $40{ }^{\circ} \mathrm{C}$. For TCCA, THM concentrations increased fivefold as the temperature increased from $25{ }^{\circ} \mathrm{C}$ to $40{ }^{\circ} \mathrm{C}$. In addition, the order of the effect of temperature on THM formation by these disinfectants follows TCCA > $\mathrm{BCDMH}>\mathrm{NaClO}$. The reaction rate of $\mathrm{NaClO}$ under different temperatures was mainly affected by two competing effects including $\mathrm{HClO}$ ionization, and reaction efficiency between $\mathrm{HClO}$ and organic matters. Accelerated reaction efficiency 
overcomes the negative effect of decreased $\mathrm{HClO}$ by ionization at high temperatures, thus results in high DBPs. For TCCA and BCDMH, the heat-promoted hydrolysis of bound chlorine/bromine becomes another important factor. For example, the $\mathrm{HClCy}^{-}$, as a main species of bound chlorine in TCCA, can be substantially hydrolyzed to form $\mathrm{HClO}$ at pool conditions under high temperatures (Wojtowicz, 1996). Compared to THMs, HAAs showed a less prominent increasing trend, which is possibly due to the low BFA concentration or thermal decarboxylation of HAAs (Lifongo et al., 2010). Kanan and Karanfil (2011) also find that the effect of temperature is more pronounced on the formation of trichloromethane than di- and trichloroacetic acid. The increasing trend of THM and HAA concentrations was consistent with the decrease of residual free chlorine/bromine (Figure 3.8). Thus, a proper temperature control in realistic swimming pools should be a tradeoff between bather comfort and DBP formation issues. Temperature reduction would be an effective way to minimize the DBP formation especially for pools preferring high temperatures, e.g. hydrotherapy and spa. Alternatively, frequent and continuous exchange of pool water can reduce DBPs from the source.
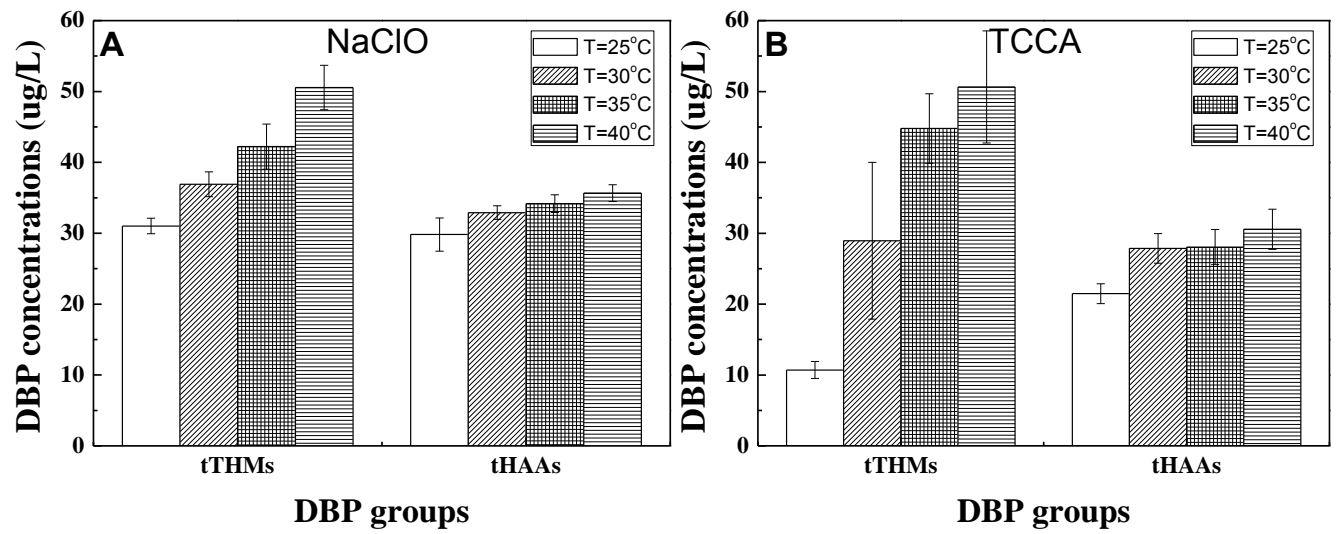


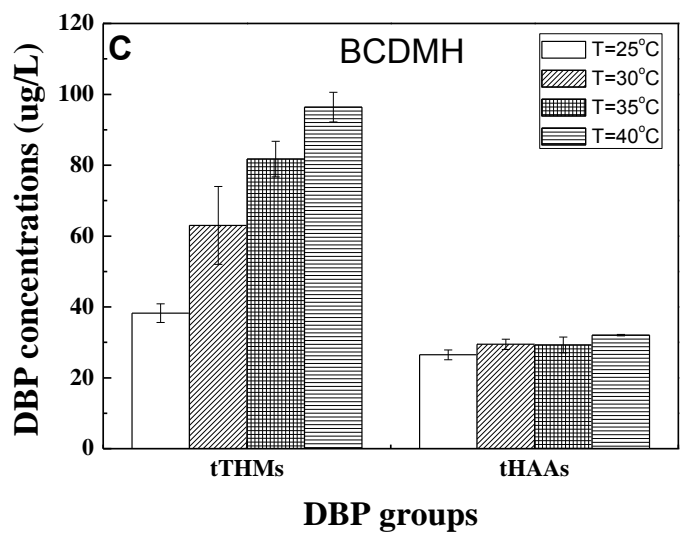

Figure 3.7 Effect of temperature on DBP formation (filling water: tap (1.14 mg/L as TOC); temperature: $25,30,35$ and $40{ }^{\circ} \mathrm{C} ; \mathrm{pH}: 7.5$; reaction time: 24 hours; free chlorine/bromine: $3 \mathrm{mg} / \mathrm{L}$ as $\mathrm{Cl}_{2}$; BFA: $1 \mathrm{mg} / \mathrm{L}$ as TOC; disinfectants: $\mathrm{NaClO} / \mathrm{TCCA} / \mathrm{BCDMH})$

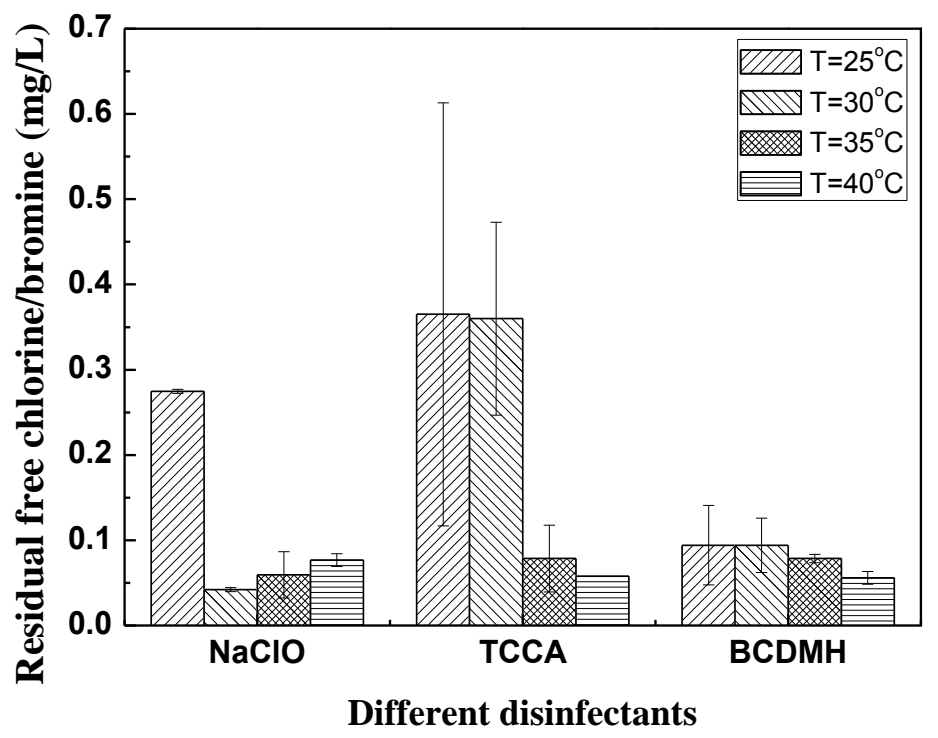

Figure 3.8 Effect of temperature on residual free chlorine/bromine (filling water: tap (1.14 mg/L as TOC); temperature: $25,30,35$ and $40{ }^{\circ} \mathrm{C}$; $\mathrm{pH}: 7.5$; reaction time: 24 hours; free chlorine/bromine: $3 \mathrm{mg} / \mathrm{L}$ as $\mathrm{Cl}_{2}$; BFA: $1 \mathrm{mg} / \mathrm{L}$ as TOC; disinfectants: $\mathrm{NaClO} / \mathrm{TCCA} / \mathrm{BCDMH})$

\subsubsection{Effect of BFA and free chlorine concentration on DBP formation}

The next batch experiments were aimed at investigating the effects of BFA and free chlorine/bromine concentrations, under the realistic/regulated range of pool water, 
on the DBP formation. BFA concentrations of 1 and $5 \mathrm{mg} / \mathrm{L}$ as TOC were used and the justification was provided in Section 3.2.3. The experiment without BFA addition $(0 \mathrm{mg} / \mathrm{L})$ was conducted as the control. Free chlorine/bromine concentrations of 1 and $3 \mathrm{mg} / \mathrm{L}$ as $\mathrm{Cl}_{2}$ were used because this concentration range is stipulated by Singapore regulation (NEA, 2005). The reaction conditions were: filling water: tap; temperature: $30{ }^{\circ} \mathrm{C} ; \mathrm{pH}: 7.5$; reaction time: 24 hours; disinfectants: $\mathrm{NaClO} / \mathrm{TCCA} / \mathrm{BCDMH}$.

Generally, an increase of free chlorine enhanced the THM and HAA formation (see Figure 3.9). Interestingly, at chlorine/bromine concentrations of $1 \mathrm{mg} / \mathrm{L}$, both THMs and HAAs decreased with increasing TOC concentrations. This may be due to the chlorine consumption by ammonia and other organic $\mathrm{N}$-compounds which are competing with precursors of THMs/HAAs. Ammonium ions, and acid amide, especially urea were reported as effective trichloramine precursors at acidic and neutral pH levels (Schmalz et al., 2011). Chloramine was also known as combined chlorine and reported in the investigated pools with levels of a few hundred ug/L (Table 3.4). Thus, urea and ammonium chloride in BFA might decrease the formation of THMs and HAAs. The finding that the chlorine/bromine demand competition between different DBP precursors can hardly be discovered and proposed if an excess of chlorine/bromine is applied for FP tests. With free chlorine/bromine concentrations of $3 \mathrm{mg} / \mathrm{L}$, THMs and HAAs first increased when TOC increased from 0 to $1 \mathrm{mg} / \mathrm{L}$ and then decreased when TOC further increased to $5 \mathrm{mg} / \mathrm{L}$ for $\mathrm{NaClO}$ and $\mathrm{BCDMH}$. This indicates that the free chlorine/bromine of 3 
$\mathrm{mg} / \mathrm{L}$ was sufficient for DBP formation when TOC was less than $1 \mathrm{mg} / \mathrm{L}$, and the chlorine/bromine competition between different precursors existed again when TOC was $5 \mathrm{mg} / \mathrm{L}$. When TCCA was applied with $3 \mathrm{mg} / \mathrm{L}$ as $\mathrm{Cl}_{2}$, there was still a decreasing trend of THMs and HAAs with increasing TOC concentrations. The stabilization property of TCCA limits the availability of free chlorine for the DBP formation. Therefore, the amount of available free chlorine/bromine is the main contributor of THM/HAA or other potential DBP formation. Furthermore, the bather load in the pool water should be controlled because components of body fluid, skin, hair etc. are important reaction partners for DBP formation (Zwiener et al., 2007), although we found in our artificial batch experiments no direct contribution to THM/HAA formation due to the existence of chlorine/bromine competition. Control of organic input can reduce the formation of other potential toxic DBPs than THM/HAA at the source. The contribution of BFA to DBP formation will be further discussed in Section 3.3.6.

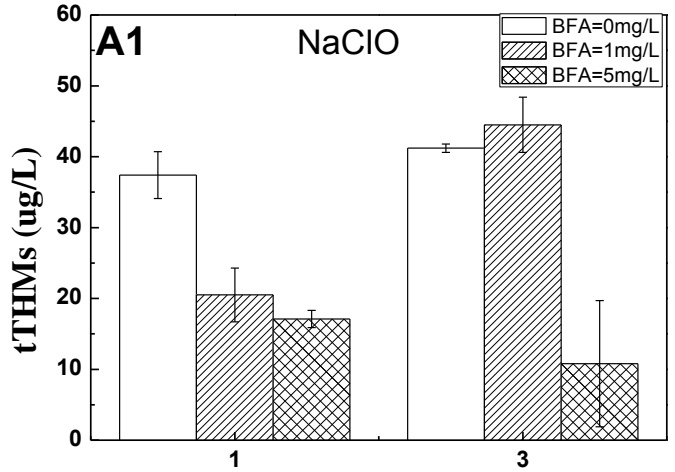

Free chlorine/bromine (mg/L)

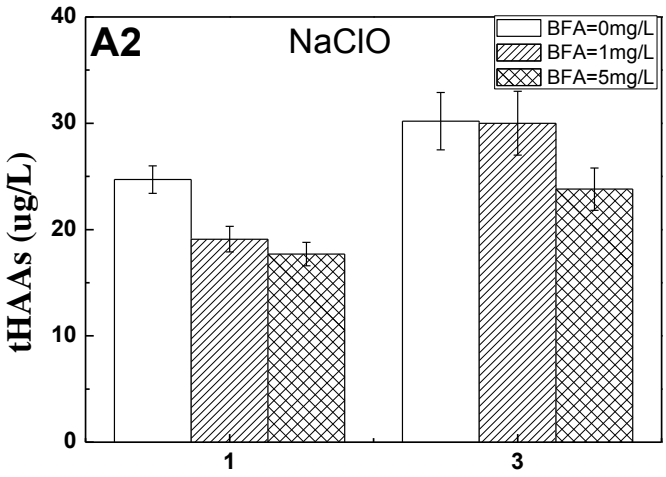

Free chlorine/bromine (mg/L) 


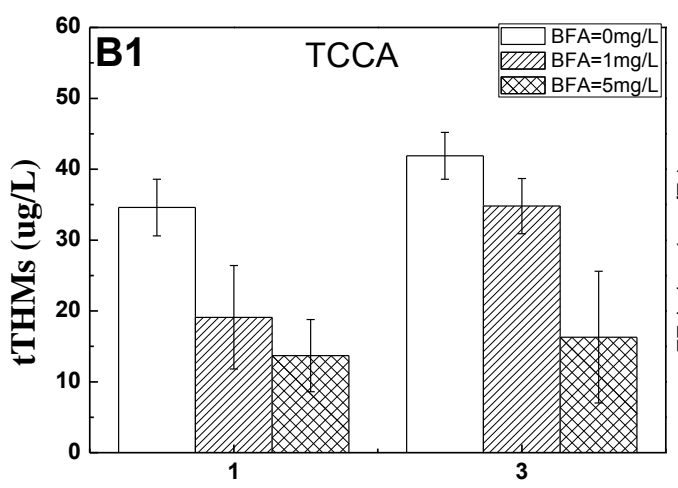

Free chlorine/bromine $(\mathrm{mg} / \mathrm{L})$

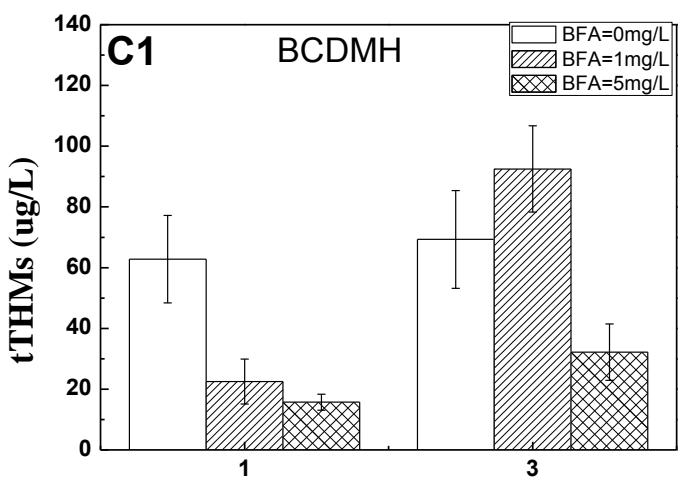

Free chlorine/bromine (mg/L)

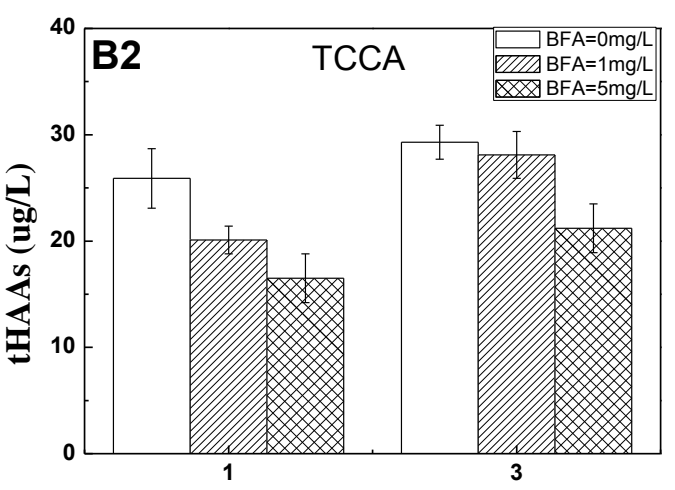

Free chlorine/bromine (mg/L)

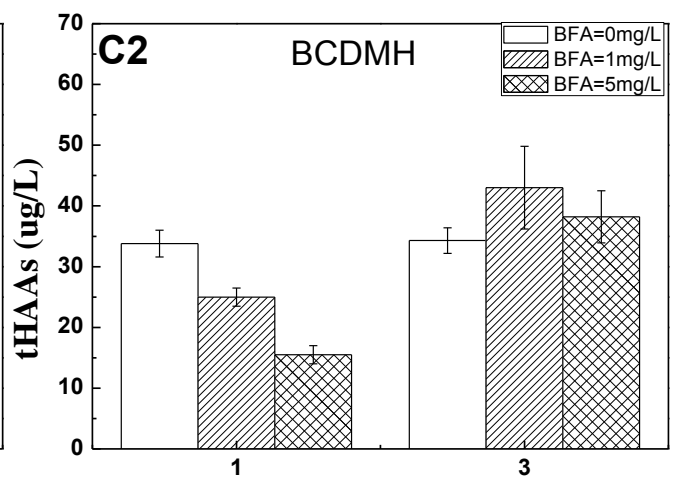

Free chlorine/bromine (mg/L)

Figure 3.9 Effect of BFA and free chlorine/bromine concentration on THM and HAA formation (filling water: tap $\left(1.27 \mathrm{mg} / \mathrm{L}\right.$ as TOC); temperature: $30^{\circ} \mathrm{C} ; \mathrm{pH}: 7.5$; free chlorine/bromine: 1 or $3 \mathrm{mg} / \mathrm{L}$ as $\mathrm{Cl}_{2}$; $\mathrm{BFA}$ : 0 or 1 or $5 \mathrm{mg} / \mathrm{L}$ as TOC; reaction time: 24 hours; disinfectants: $\mathrm{NaClO} / \mathrm{TCCA} / \mathrm{BCDMH}$ )

\subsubsection{Comparison of NOM and BFA on DBP formation}

The respective effect of NOM and BFA on DBP formation was investigated under defined conditions: temperature: $30{ }^{\circ} \mathrm{C} ; \mathrm{pH}: 7.5$, reaction time: 24 hours; free chlorine/bromine: $3 \mathrm{mg} / \mathrm{L}$ as $\mathrm{Cl}_{2}$; disinfectants: $\mathrm{NaClO} / \mathrm{TCCA} / \mathrm{BCDMH}$. Both BFA stock solution and tap water were diluted with buffer solutions until TOC was $1 \mathrm{mg} / \mathrm{L}$. DBP formation potential of individual components, including urea, creatinine, uric acid, citric acid, L-histidine, hippuric acid and ammonium chloride, in BFA was also investigated. The conditions were: filling water: deionized (DI) water; temperature: 
$30{ }^{\circ} \mathrm{C}$; $\mathrm{pH}$ : 7.5, reaction time: 24 hours; free chlorine: $3 \mathrm{mg} / \mathrm{L}$ as $\mathrm{Cl}_{2}$; ammonium chloride as $\mathrm{TN}$ and other individual component as TOC: $1 \mathrm{mg} / \mathrm{L}$; disinfectants: $\mathrm{NaClO}$.

Figure 3.10 shows that NOM in tap water exhibited higher reactivity in producing THMs while BFA predominantly promoted the formation of HAAs regardless of which disinfectants were used. The consistent finding of these three disinfectants comes from the fact that $\mathrm{HClO} / \mathrm{HBrO} / \mathrm{ClO}^{-} / \mathrm{BrO}^{-}$are the only active ingredients for DBP formation. The difference of DBP species is possibly caused by the different constituents in NOM and BFA. The TOC in natural waters is generally dominated by humic and fulvic acids which were reported to be the main contributors of THMs (Scully et al., 1988). The components, such as uric acid, citric acid and hippuric acid were individually verified as main precursors of HAA formation (Figure 3.11), which could partially explain the high HAA occurrence (191-1906 $\mu \mathrm{g} / \mathrm{L}$, Table 3.4) in Singapore swimming pools: 1) Continually introduced body fluids by swimmers can react with the continually dosed chlorine to produce HAAs; 2) Limited pool water is replaced by fresh water so that the retention time of pool water is rather long, and the pool water treatment rarely removes the DBPs; 3) The HAA characters, including polar and non-volatile, cause a longer residence time in the water phase, compared to the volatile THMs. The relatively low THM and high HAA concentrations generated from individual compounds of BFA compared to mixture BFA (Table 3.8) may result from the effect of the mixed matrix. 


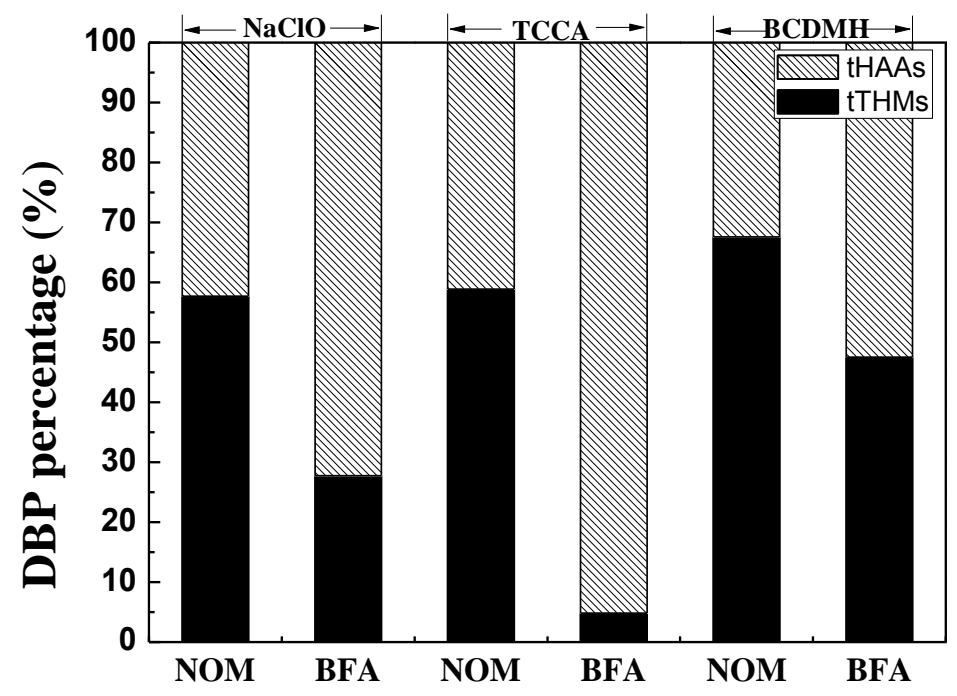

Figure 3.10 Effect of NOM and BFA on DBP formation (temperature: $30{ }^{\circ} \mathrm{C} ; \mathrm{pH}$ : 7.5, reaction time: 24 hours; free chlorine/bromine: $3 \mathrm{mg} / \mathrm{L}$ as $\mathrm{Cl}_{2}$; TOC: $1 \mathrm{mg} / \mathrm{L}$; disinfectants: $\mathrm{NaClO} / \mathrm{TCCA} / \mathrm{BCDMH})$

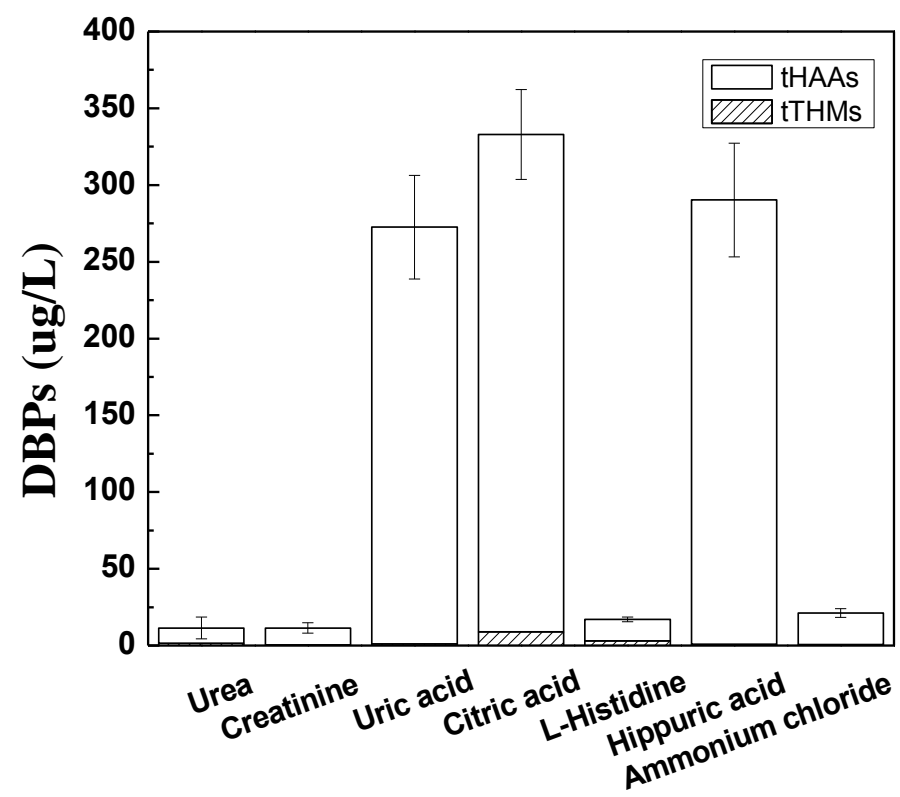

Figure 3.11 DBP formation potential of individual component in BFA (filling water: DI; temperature: $30^{\circ} \mathrm{C} ; \mathrm{pH}: 7.5$, reaction time: 24 hours; free chlorine: $3 \mathrm{mg} / \mathrm{L}$ as $\mathrm{Cl}_{2}$; ammonium chloride as TN and other individual component as TOC: $1 \mathrm{mg} / \mathrm{L}$; disinfectants: $\mathrm{NaClO}$ ) 
Table 3.8 Comparison of NOM and BFA on DBP formation (temperature: $30{ }^{\circ} \mathrm{C} ; \mathrm{pH}$ : 7.5, reaction time: 24 hours; free chlorine/bromine: $3 \mathrm{mg} / \mathrm{L}$ as $\mathrm{Cl}_{2} ; \mathrm{NOM}$ or BFA: 1 $\mathrm{mg} / \mathrm{L}$ as TOC; disinfectants: $\mathrm{NaClO} / \mathrm{TCCA} / \mathrm{BCDMH})$

\begin{tabular}{lllll}
\hline & & tTHMs & $\begin{array}{r}\text { tHAAs } \\
\mu \mathrm{g} / \mathrm{L}\end{array}$ & Total \\
\hline \multirow{2}{*}{$\mathrm{NaClO}$} & NOM & $32.4 \pm 0.5$ & $23.8 \pm 2.1$ & $56.2 \pm 2.6$ \\
& BFA & $8.0 \pm 0.6$ & $20.8 \pm 3.5$ & $28.8 \pm 4.1$ \\
\multirow{2}{*}{ TCCA } & NOM & $33.0 \pm 2.6$ & $23.1 \pm 1.5$ & $56.1 \pm 4.1$ \\
& BFA & $0.8 \pm 0.4$ & $15.8 \pm 2.3$ & $16.6 \pm 2.7$ \\
\multirow{2}{*}{ BCDMH } & NOM & $56.4 \pm 12.7$ & $27.0 \pm 1.7$ & $83.4 \pm 14.3$ \\
& BFA & $26.0 \pm 7.4$ & $28.7 \pm 1.6$ & $54.7 \pm 9.0$ \\
\hline
\end{tabular}

Note:

Standard deviation was based on at least duplicated samples.

\subsection{Conclusions}

Two alternatives known as stabilized disinfectants, TCCA and BCDMH, were for the first time investigated and compared to the most commonly used disinfectant $\mathrm{NaClO}$ in terms of chlorine/bromine consumption and $\mathrm{DBP}$ formation under simulated tropical pool conditions. The conclusions from this study are as follows:

1) UV radiation from the sunlight played an important role in high chlorine degradation and resulted in low residual chlorine in pool water.

2) TCCA showed higher residual free chlorine concentrations and the lower DBP formation compared to $\mathrm{NaClO}$, indicating its feasibility as stabilized chlorine.

3) Residual chlorine/bromine and THM/HAA formation depended mainly on the total chlorine/bromine dosage instead of the dosing methods (continuous vs. shock, $3 \times 5 \mathrm{mg} / \mathrm{L}$ vs. $15 \mathrm{mg} / \mathrm{L}$ ) if the reaction time was sufficient. Two-stage second-order-kinetic-based models provided good fitting for both chlorine decay and THM/HAA formation. 
4) Higher temperature accelerated free chlorine/bromine consumption and promoted DBP formation.

5) Chlorine/bromine competition existed between the precursors of THM/HAA and other DBPs when high TOC or TN was available but free chlorine/bromine was limited.

6) Different constituents in NOM and BFA contributed to the diverse species of DBPs. Uric acid, citric acid and hippuric acid as components in BFA were individually verified to be the main precursors for HAA formation. 


\section{Chapter 4 Removal of Haloacetic Acids from Swimming Pool}

\section{Water by Reverse Osmosis and Nanofiltration}

\subsection{Introduction}

Swimming is a popular activity for exercise and entertainment. Disinfection by chlorine gas, sodium hypochlorite or calcium hypochlorite is commonly practiced to keep SPW microbiologically safe and hygienic (WHO, 2006). However, these disinfectants can react with water constituents of natural and anthropogenic origin (e.g., NOM and body fluid) to produce toxic DBPs (Fischer et al., 2012). HAAs are a prevalent class of DBPs characterized by their high frequency of occurrence, considerable concentrations and potent toxicity. HAAs are mutagenic in bacteria, and induce DNA damage and chromosomal aberrations in mammalian cells in vitro (IARC, 2004; Richardson et al., 2007). Besides, some HAAs cause liver tumors, leukemias and abdominal cavity mesotheliomas in experimental animals (Richardson et al., 2007). These concerns led to the regulation over HAAs for DWs by EPA in 1998, with a MCL of $60 \mu \mathrm{g} / \mathrm{L}$ for the sum of five HAAs (EPA, 1998). However, so far no regulation has been enacted for HAAs in SPWs. The presence of HAAs in SPWs has also raised significant concerns, with recent studies reporting their HAA concentrations of more than an order of magnitude higher than the MCL in drinking water (Wang et al., 2014; Yang et al., 2016). HAAs are selected as the targeted contaminants of DBPs in SPW treatment due to the following aspects: 1) high concentrations; 2) potent toxicity; 3) stable physicochemical property (polar 
and non-volatile); 4) systematic changes in the molecular structures (sharing the same acetic acid structure with varied degrees of halogenation).

High HAA accumulation in SPWs reveals that the traditional treatment system of "flocculation-filtration-disinfection" is insufficient. Thus, more effective technologies are needed for HAA removal. There are several reports about the biodegradation of HAAs in DWs systems (Bayless and Andrews, 2008; Grigorescu et al., 2010; Zhang et al., 2009). However, extrapolation of such technology to SPW treatment may raise uncertainties due to its distinguishing water matrix compared to DWs. Photodegradation and thermal degradation are two possible HAA treatment methods while still encountering the problems of complex post-processing (e.g., the removal of the titanium dioxide suspensions) and/or high heating cost (Lifongo et al., 2004; Lifongo et al., 2010).

$\mathrm{RO}$ and NF have been widely used for the treatment of trace organic compounds in water and wastewater (Doederer et al., 2014; Dong et al., 2016; Kimura et al., 2003; Nghiem et al., 2004; 2005; Verliefde et al., 2008). The high efficiency in rejection and simple operation make them promising candidates for the potential removal of trace HAAs from SPWs. There have been a handful of studies reporting the effective rejection of HAAs (> 90\%) by membranes under conditions of interest (Chalatip et al., 2009; Kimura et al., 2003). Nevertheless, the detailed rejection mechanisms and controlling factors are yet to be systematically investigated. 
The systematic changes in the molecular structures of HAAs (sharing the same acetic acid structure with varied degrees of halogenation) prompt us to perform a comprehensive study on their removal by membranes. Specifically, we tested the rejection performance of 9 HAAs and 7 surrogate molecules by four RO/NF membranes under different water chemistry conditions. By correlating their rejections to various physicochemical properties of membranes and solutes, mechanisms governing HAA rejections were revealed in detail.

\subsection{Materials and methods}

\subsubsection{Chemicals and materials}

General chemicals. All the general chemicals used in this study were analytical grade (> 99\%), unless otherwise specified. Sodium hydroxide $(\mathrm{NaOH})$, hydrochloric acid $(\mathrm{HCl}, 37 \%)$ and sodium chloride $(\mathrm{NaCl})$ were obtained from Merck. MilliQ water was used in all stock solution preparations and experiments (Millipore, Billerica, MA).

HAAs. Nine HAAs were tested as shown in Table 4.1. HAA standards were analytical grade with $\geq 97 \%$ purity (Sigma-Aldrich). Solvents, including MTBE and methanol, were GC grade. Other chemicals for HAA quantification, e.g., sulfuric acid (98\%), sodium bicarbonate, copper sulfate and sodium sulfate, were at least ACS reagents. 
Surrogate molecules. Seven surrogates, namely glycerol, erythritol, xylose, glucose, maltose, sucrose and raffinose, were analytical grades with $\geq 99 \%$ purity. All surrogates were obtained from Sigma-Aldrich except sucrose from USB.

Table 4.1 Haloacetic acid and surrogate properties

\begin{tabular}{|c|c|c|c|c|c|c|}
\hline Name & Abbreviation & Structure & $\begin{array}{l}\text { Molecular } \\
\text { weight } \\
\mathrm{g} / \mathrm{mol}\end{array}$ & $\mathrm{pKa}^{\mathrm{a}}$ & $\begin{array}{l}\text { Diffusivity } \\
10^{-9} \mathrm{~m}^{2} / \mathrm{s}\end{array}$ & $\begin{array}{l}\text { Stokes radius }{ }^{\mathrm{c}} \\
\mathrm{nm}\end{array}$ \\
\hline \multicolumn{7}{|c|}{ HAAs } \\
\hline Chloroacetic acid & MCAA & Chain & 94.5 & 2.65 & 1.177 & 0.207 \\
\hline Bromoacetic acid & MBAA & Chain & 139.0 & 2.73 & 1.158 & 0.211 \\
\hline Dichloroacetic acid & DCAA & Chain & 128.9 & 1.37 & 1.032 & 0.237 \\
\hline Bromochloroacetic acid & BCAA & Chain & 173.4 & 1.39 & 1.018 & 0.240 \\
\hline Dibromoacetic acid & DBAA & Chain & 217.8 & 1.47 & 1.004 & 0.243 \\
\hline Trichloroacetic acid & TCAA & Chain & 163.4 & 0.09 & 0.926 & 0.264 \\
\hline Tribromoacetic acid & TBAA & Chain & 296.8 & 0.22 & 0.895 & 0.273 \\
\hline $\begin{array}{l}\text { Dibromochloroacetic } \\
\text { acid }\end{array}$ & DBCAA & Chain & 252.3 & 0.13 & 0.905 & 0.270 \\
\hline $\begin{array}{l}\text { Bromodichloroacetic } \\
\text { acid }\end{array}$ & BDCAA & Chain & 207.8 & 0.05 & 0.915 & 0.267 \\
\hline \multicolumn{7}{|c|}{ Surrogates } \\
\hline Glycerol & -- & Chain & 92.1 & 14.15 & 1.091 & 0.224 \\
\hline Erythritol & -- & Chain & 122.1 & 13.27 & 0.929 & 0.263 \\
\hline Xylose & -- & Circular & 150.1 & 12.15 & 0.842 & 0.290 \\
\hline Glucose & -- & Circular & 180.2 & 12.28 & 0.755 & 0.323 \\
\hline Maltose & -- & Circular & 342.3 & 11.94 & 0.511 & 0.478 \\
\hline Sucrose & -- & Circular & 342.3 & 12.62 & 0.511 & 0.478 \\
\hline Raffinose & -- & Circular & 504.4 & 12.74 & 0.418 & 0.585 \\
\hline
\end{tabular}

Note:

a Data from references (Bhattacharyya et al., 2012; Kong et al., 2014).

b The diffusivity was calculated by the Wilke-Chang equation (Eqs. (4.2) and (4.3)) at $25{ }^{\circ} \mathrm{C}$ (Geankoplis, 1993).

${ }^{\mathrm{c}}$ Stokes radius was calculated by Stokes-Einstein equation (Eq. (4.1)) (Deen, 1987).

RO/NF membranes. Four commercial flat sheet RO/NF membranes, including two NF membranes (NF90 and NF270) and two RO membranes (XLE and SB50), were investigated. SB50 was from TriSep with cellulose acetate as an active surface layer and the others were from Dow Filmtec with polyamide as the selective layer. All these membranes were stored at $4{ }^{\circ} \mathrm{C}$ in the dark. The virgin membrane properties, 
including water permeability, $\mathrm{NaCl}$ rejection, $\mathrm{MWCO}, \zeta$ potential (at $\mathrm{pH} 7.5$ ), pore radius, and roughness, are listed in Table 4.2. 
Table 4.2 Membrane properties

\begin{tabular}{|c|c|c|c|c|c|c|c|c|c|}
\hline Membrane & Type & $\begin{array}{l}\text { Surface layer } \\
\text { material }\end{array}$ & Manufacturer & $\begin{array}{l}\text { Water } \\
\text { permeability } \\
\mathrm{L} / \mathrm{m}^{2} \mathrm{~h} \text { bar }\end{array}$ & $\begin{array}{l}\mathrm{NaCl} \\
\text { Rejection } \\
\% \\
\%\end{array}$ & $\begin{array}{l}\text { MWCO } \\
\mathrm{Da}\end{array}$ & $\begin{array}{l}\zeta \text { potential } \\
\text { at } \mathrm{pH} 7.5^{\mathrm{e}} \\
\mathrm{mV}\end{array}$ & $\begin{array}{l}\text { Pore } \\
\text { radius }^{\mathrm{f}} \\
\mathrm{nm}\end{array}$ & $\begin{array}{l}\text { RMS } \\
\text { roughness } \\
\text { nm }\end{array}$ \\
\hline XLE & RO & Polyamide & Dow Filmtec & 7.25 & 91.2 & 96 & -33 & 0.30 & $129.5 \pm 23.4$ \\
\hline NF90 & NF & Polyamide & Dow Filmtec & 7.69 & 82.7 & $118,180^{\mathrm{c}}, 200^{\mathrm{d}}$ & -33 & 0.31 & $142.8 \pm 9.6$ \\
\hline NF270 & NF & Polyamide & Dow Filmtec & 16.10 & 30.6 & $266,340^{\mathrm{c}}, 300^{\mathrm{d}}$ & -36 & 0.44 & $9.0 \pm 4.2$ \\
\hline SB50 & RO & $\begin{array}{l}\text { Cellulose } \\
\text { acetate }\end{array}$ & TriSep & 2.18 & 75.8 & 152 & -13 & 0.33 & NA \\
\hline
\end{tabular}

Note:

${ }^{\text {a }}$ Water permeability was obtained by compacting the membranes for $24 \mathrm{~h}$ under the defined conditions $\left(100 \mathrm{psi}, 25^{\circ} \mathrm{C}, \mathrm{pH} \sim 6.7\right.$, MilliQ water).

${ }^{\mathrm{b}} \mathrm{NaCl}$ rejection was determined after $\mathrm{NaCl}$ was dosed to the feed tank for $24 \mathrm{~h}$ under defined conditions $\left(100 \mathrm{psi}, 25^{\circ} \mathrm{C}, \mathrm{pH} \sim 6.7,0.05 \mathrm{M} \mathrm{NaCl}\right)$.

${ }^{c}$ Data from López-Muñoz et al. (2009), using PEGs with molecular weights of 62, 200, 300, 600, and 1500 Da as the probing molecules.

${ }^{\mathrm{d}}$ Data from Do et al. (2012), using polyethylene glycols with molecular weights of 200, 400 and 600 Da as the probing molecules.

${ }^{\mathrm{e}}$ Zeta potential values at $\mathrm{pH} 7.5$ were interpolated from Figure 4.4.

${ }_{\mathrm{f}}^{\mathrm{f}}$ Pore radius was calculated according to Nghiem et al. (2004) (see details in Appendix A1 and Table 4.3).

g Data from Tang et al. (2009). 


\subsubsection{Membrane characterization}

FE-SEM. Field emission scanning electron microscopy (FE-SEM, JSM-7600F) was used to characterize the topography of membrane surfaces at an accelerating voltage of $2 \mathrm{keV}$. The wet membranes were dried in a vacuum at an ambient temperature (25 $\pm 1{ }^{\circ} \mathrm{C}$ ). Before measurements, the membrane surface was coated with a layer of platinum by a sputter coater (Emitech SC7620, Quorum, UK) (Zhang et al., 2014).

ATR-FTIR. The chemical bonds of the membranes were characterized by attenuated total reflection fourier transform infrared spectroscopy (ATR-FTIR, Shimadzu IR Prestige 21). The spectrum was obtained from an average of 45 scans with a resolution of $4 \mathrm{~cm}^{-1}$ within the scanning range of $400-4000 \mathrm{~cm}^{-1}$ under absorbance mode.

Zeta potential. Membrane surface charge quantified as zeta potential was measured by an electrokinetic analyzer (SurPASS, Anton Paar GmbH, Austria). The channel height of two $20 \times 10 \mathrm{~mm}$ adjustable gap cells was kept at $100-150 \mu \mathrm{m}$. The measurement was performed in $0.05 \mathrm{M} \mathrm{NaCl}$ as a background electrolyte over a $\mathrm{pH}$ range of $\sim 2-11$ at an ambient temperature $\left(25 \pm 1{ }^{\circ} \mathrm{C}\right)$. The $\mathrm{NaCl}$ electrolyte solution was first manually adjusted to $\mathrm{pH} \sim 11$ by $1 \mathrm{M} \mathrm{NaOH}$. The $\mathrm{pH}$ was decreased with an interval of $\sim 0.5$ by automatic titration with $0.05 \mathrm{M} \mathrm{HCl}$ during the entire

measurement. Zeta potential was computed according to the HelmholtzSmoluchowski (HS) equation (Chun et al., 2003). 


\subsubsection{Membrane filtration experiments}

Membrane setup. The bench scale RO/NF filtration system consisted of four identical rectangular cross-flow CF042 cells (Delrin Acetal, Sterlitech, Kent, WA, USA) (see Figure 4.1). Each cell held an active membrane area of $42 \mathrm{~cm}^{2}(4.6 \mathrm{~cm} \times$ $9.2 \mathrm{~cm}$ ) and possessed a spacer with a thickness of $1.2 \mathrm{~mm}$ (GE Osmonics, Minnetonka, MN, USA). Both permeate and retentate were recycled back to the feed tank (20 L) to keep a constant feed concentration. The temperature was maintained at $25^{\circ} \mathrm{C}$ by a chiller (Polyscience, Niles, IL, USA).

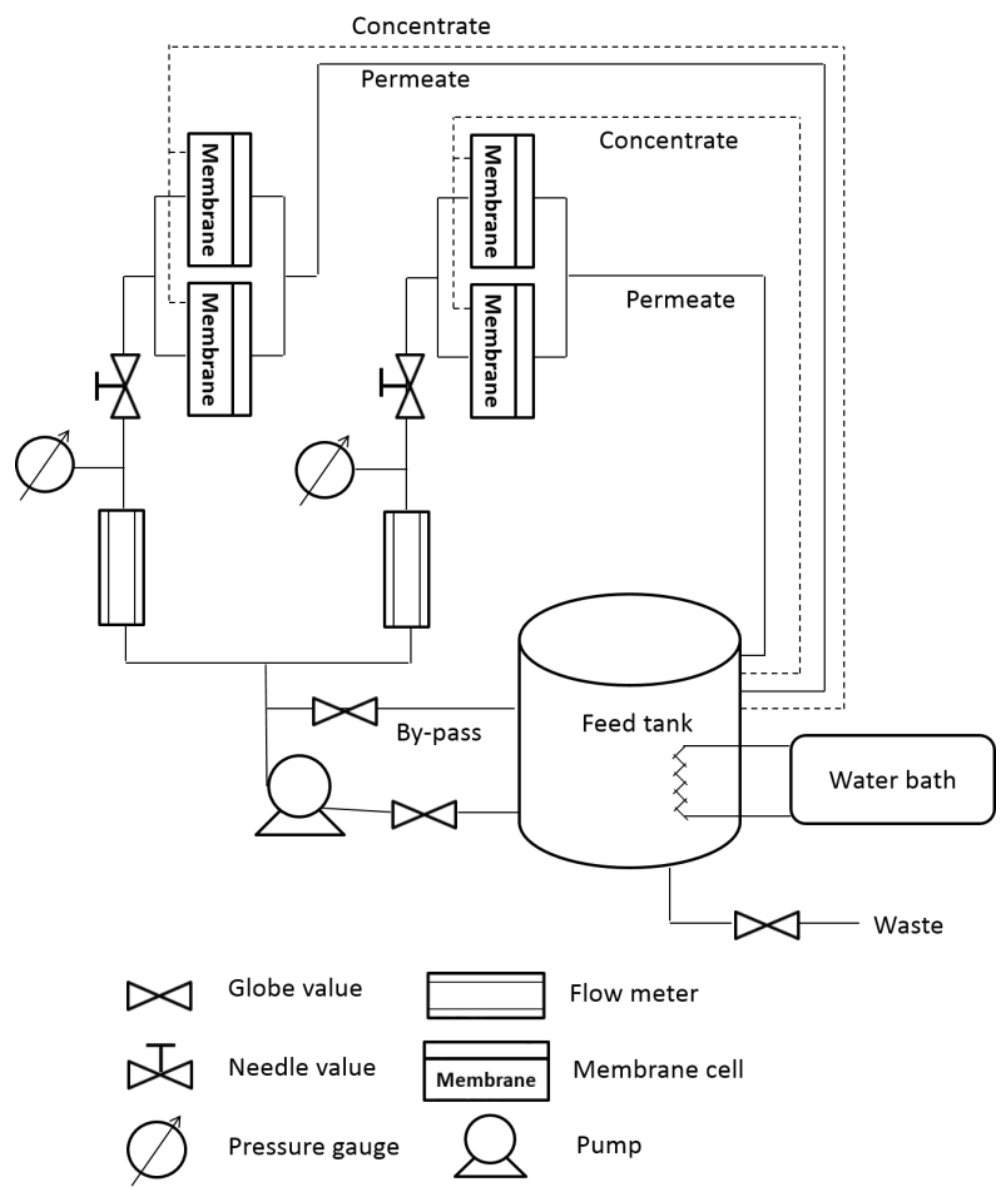

Figure 4.1 Schematic diagram of the membrane filtration setup 
Permeability, salt rejection and HAA rejection. The virgin membrane coupons were soaked in the MilliQ water for $24 \mathrm{~h}$ before being loaded into the test cells. The membranes were filtered with MilliQ water for at least $24 \mathrm{~h}$ until the flux remained constant to eliminate the effect of membrane compaction. The rejection tests were performed using a feed water containing $0.05 \mathrm{M} \mathrm{NaCl}$ (reflecting the typical salinity for local SPWs as shown in Table 5.4, sodium concentration reached at $1062 \pm 90$ $\mathrm{mg} / \mathrm{L}$ based on our preliminary test) and $100 \mu \mathrm{g} / \mathrm{L}$ of each HAA. Filtration tests were performed at $100 \mathrm{psi}$ at a fixed cross-flow of $0.8 \mathrm{~L} / \mathrm{min}$ (corresponding to $18.1 \mathrm{~cm} / \mathrm{s}$ cross-flow velocity) under a constant temperature of $25^{\circ} \mathrm{C}$. Preliminary experiments showed that pressures higher than 100 psi could enhance the concentration polarization effect and therefore was discarded. The $\mathrm{pHs}$ of the feed were adjusted to $3.5,5.5$, or 7.5 by the addition of $0.1 \mathrm{M} \mathrm{HCl}$ or $\mathrm{NaOH}$. To eliminate the minor difference caused by membrane materials, the same membrane coupons were used in different $\mathrm{pH}$ conditions. Upon changes of $\mathrm{pHs}$, the system was run for at least $2 \mathrm{~h}$ to ensure system stabilization. Both feed and permeate were collected for HAA analysis. Water flux was monitored manually by weighting the mass of permeate at a predetermined time interval. Conductivity was continually measured by a conductivity meter (Myron L's Ultrameter II 4P) for the calculation of salt rejection.

MWCO and membrane pore radius. The surrogates used in the current study were neutral hydrophilic compounds (Table 4.1) to ensure that their rejections were primarily based on size exclusion. The molecular weight (MW) of these surrogates spans over $92-504 \mathrm{~g} / \mathrm{mol}$, which allows them to be used as molecular probes for 
examining pore properties of the RO/NF membranes. Rejection tests for surrogates were performed at a feed concentration of $200 \mathrm{mg} / \mathrm{L}$ of each surrogate without $\mathrm{pH}$ adjustment ( pH 6.7). Additional solution pHs of 3.5, 5.5, and 7.5 were included for verification purpose. $\mathrm{NaCl}$ was not added in these rejection tests to avoid its interference with the surrogate analysis by HPLC-RID. The MWCO for each membrane was determined as the MW corresponding to a $90 \%$ solute rejection by interpolating the surrogate rejection curve.

Molecular radii of both surrogates and HAAs were calculated according to the Stokes-Einstein equation (Eq. (4.1)) based on the assumption of spherical solutes (Deen, 1987; Einstein, 1956). It should be noted that the actual molecular shape in the solution can be non-sphere which may somehow affect the rejection evaluation and different orientations of a non-sphere molecule in a pore may influence its rejection behavior as well (Deen, 1987; Kiso et al., 2010). The diffusivity in Eq. (4.1) was obtained from Wilke-Chang equation (Geankoplis, 1993) as shown in Eq. (4.2) or (4.3):

$$
\begin{gathered}
r_{s}=\frac{k T}{6 \pi \eta_{B} D_{A B}} \\
D_{A B}=1.173 \times 10^{-16}\left(\Phi M_{B}\right)^{0.5} \frac{T}{\eta_{B} V_{A}^{0.6}} \quad\left(\text { for } V_{A} \leq 0.5 \mathrm{~m}^{3} / \mathrm{kmol}\right) \\
D_{A B}=\frac{9.96 \times 10^{-16} T}{\eta_{B} V_{A}^{1 / 3}}\left(\text { for } V_{A}>0.5 \mathrm{~m}^{3} / \mathrm{kmol}\right)
\end{gathered}
$$

where $D_{A B}$ is the diffusivity in the bulk solution $\left(\mathrm{m}^{2} / \mathrm{s}\right), k$ is the Boltzmann constant $(\mathrm{J} / \mathrm{K}), T$ is the absolute temperature $(\mathrm{K}), \eta_{B}$ is the solvent viscosity $(\mathrm{Pa} \cdot \mathrm{s}), r_{s}$ is the 
solute radius (m), $\Phi$ is the association parameter of the solvent (dimensionless), $M_{B}$ is the molecular weight of the solvent $(\mathrm{g} / \mathrm{mol})$, and $V_{A}$ is the solute molar volume at the boiling point $\left(\mathrm{m}^{3} / \mathrm{kmol}\right)$.

Table 4.3 Membrane pore radius estimation

\begin{tabular}{lllcl}
\hline & \multicolumn{4}{c}{ Physical parameters } \\
& NF90 & NF270 & XLE & SB50 \\
\hline Thickness (nm) & $218^{\mathrm{a}}$ & $21^{\mathrm{a}}$ & $200^{\mathrm{b}}$ & $200^{\mathrm{b}}$ \\
Porosity & $0.26^{\mathrm{a}}$ & $0.02^{\mathrm{a}}$ & $0.1^{\mathrm{c}}$ & $0.1^{\mathrm{c}}$ \\
& & & Pore radius $r_{p}(\mathrm{~nm})$ & \\
Glycerol & 0.30 & 0.42 & 0.29 & 0.30 \\
Erythritol & 0.32 & 0.45 & 0.31 & 0.33 \\
Xylose & 0.32 & 0.45 & 0.30 & 0.35 \\
Glucose & -- & 0.45 & -- & -- \\
Average & 0.31 & 0.44 & 0.30 & 0.33 \\
\hline
\end{tabular}

Note:

${ }^{\mathrm{a}}$ Data from Semião and Schäfer (2013).

${ }^{\mathrm{b}}$ For normal NF and RO membranes, the range of thickness is 100-300 $\mathrm{nm}$ according to Tang (2006), thus the average value $200 \mathrm{~nm}$ was used in this study.

${ }^{\mathrm{c}}$ Since the porosity of several TFC membranes were reported between 0.02-0.32 (Semião and Schäfer, 2013), we assumed the porosity for XLE and SB50 was 0.1.

To estimate the average pore radius of each membrane, we used the pore transport model developed by Nghiem et al. (2004). This model assumes size exclusion as the sole mechanism for rejection. By treating the membrane as a rejection layer which consists of parallel cylindrical pores of identical radius, both convective and diffusive transport of the solutes can be modeled. The thickness and porosity of NF90 and NF270 membranes were extracted directly from the existing literature (Semião and Schäfer, 2013). For XLE and SB50, the thickness of $200 \mathrm{~nm}$ and porosity of 0.1 were estimated based on the ranges of $100-300 \mathrm{~nm}$ for thickness and $0.02-0.32$ for porosity, respectively, for the TFC NF/RO membranes (Semião and Schäfer, 2013; Tang, 2006). The membrane pore radius was estimated using the solute transport 
model (refer to Appendix A1) by the iterative method with the restrictive condition of $\left|\Delta R_{r}\right| \leq 1 \%$, which restricted the error between the measured and calculated rejections of the solutes less than $1 \%$ (see Table 4.3). Detailed information on the calculation of membrane pore radius is presented in Appendix A1.

\subsubsection{Analytical methods}

HAA quantification. HAAs were analyzed based on a modified EPA 552.3 Method (Domino et al., 2003). The sample ( $40 \mathrm{~mL}$ ) was acidified with $2 \mathrm{~mL}$ concentrated $\mathrm{H}_{2} \mathrm{SO}_{4}(98 \%)$ to reach $\mathrm{pH} \leq 0.5$. The $\mathrm{CuSO}_{4}(2 \mathrm{~g})$ and $\mathrm{Na}_{2} \mathrm{SO}_{4}(16 \mathrm{~g})$ were added to achieve a clear phase separation and a saturated solution, respectively. The HAAs were extracted by adding $4 \mathrm{~mL}$ MTBE followed by 30 min shaking. The MTBE extract $(3 \mathrm{~mL})$ with the addition of $1 \mathrm{~mL}$ acidic methanol $\left(10 \% \mathrm{H}_{2} \mathrm{SO}_{4}\right.$ in methanol) was heated at $50{ }^{\circ} \mathrm{C}$ for 1.5 hours for HAA derivatization. The extract was quickly cooled down by the ice bath. Solution neutralization was completed by the addition of saturated $\mathrm{NaHCO}_{3}(4 \mathrm{~mL})$ followed by 2 min shaking before venting the $\mathrm{CO}_{2}$. After 1 min standing for phase separation, the methylated HAAs $(1 \mathrm{~mL})$ was extracted into a $2 \mathrm{~mL}$ vial. The methylated HAAs were quantified by GCMS (7890B GC, 5977A MS, Agilent) coupled with an HP-5MS UI column (J \& W Scientific, (5\% - phenyl) - methylpolysiloxane, $30 \mathrm{~m} \times 0.25 \mathrm{~mm} \mathrm{ID}, 0.25 \mu \mathrm{m}$ film thickness). The oven program was controlled as: 1) hold $35^{\circ} \mathrm{C}$ for $9 \mathrm{~min}$; 2) increase to $150{ }^{\circ} \mathrm{C}$ by $\left.10{ }^{\circ} \mathrm{C} / \mathrm{min} ; 3\right)$ increase to $250^{\circ} \mathrm{C}$ by $20^{\circ} \mathrm{C} / \mathrm{min}$. The temperatures of the transfer line, MS quadrupole and ion source were 280,150 and $300{ }^{\circ} \mathrm{C}$, respectively. The sample $(1 \mu \mathrm{L})$ was injected by an auto-sampler into the GCMS in a splitless mode 
and run for $25.5 \mathrm{~min}$ (with $5.40 \mathrm{~min}$ solvent delay) with a constant flow of the carrier gas (helium, $0.6 \mathrm{~mL} / \mathrm{min}$ ). The MS scanned at a range of $\mathrm{m} / \mathrm{z}$ 50-300 amu with a rate of $5.5 \mathrm{scans} / \mathrm{sec}$ under electron ionization mode at $70 \mathrm{eV}$. The quality control of this method has been described in detail in Section 3.2.1.

Surrogate quantification. The surrogates were analyzed by high-performance liquid chromatography coupled with refractive index detector (HPLC-RID, Agilent 1260 Infinity) with Hi-Plex $\mathrm{Pb}(7.7 \times 300 \mathrm{~mm}, 8 \mu \mathrm{m}) /(7.7 \times 50 \mathrm{~mm}, 8 \mu \mathrm{m})$ as guard/analytical columns. The $20 \mathrm{uL}$ sample was injected with freshly prepared ultra-pure water as the mobile phase at a flow rate of $0.25 \mathrm{~mL} / \mathrm{min}$. The column and RID temperatures were kept at $70{ }^{\circ} \mathrm{C}$ and $55^{\circ} \mathrm{C}$, respectively. The samples were run for $65 \mathrm{~min}$, and analyzed and quantified using a calibration curve fitted by at least 6 standard points between 1 and $200 \mathrm{mg} / \mathrm{L}$. The chromatogram of a standard with a concentration of $200 \mathrm{mg} / \mathrm{L}$ is shown in Figure 4.2. The retention time of each surrogate was $32.360,33.605,35.710,39.590,42.817,56.724$ and 59.799 min for raffinose, sucrose, maltose, glucose, xylose, glycerol and erythritol, respectively. 


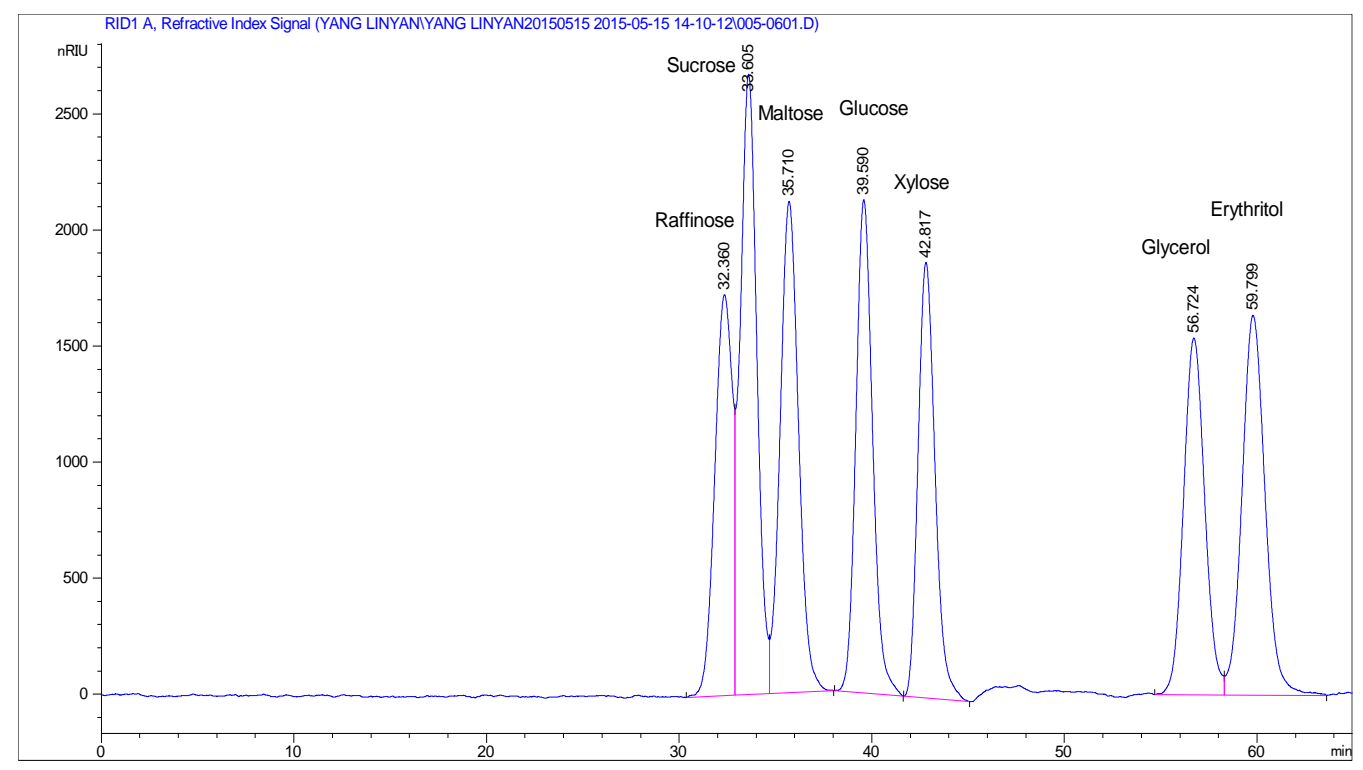

Figure 4.2 Chromatogram of a standard with a concentration level of $200 \mathrm{mg} / \mathrm{L}$ by HPLC-RID.

\subsection{Results and discussion}

\subsubsection{Membrane properties}

Figure 4.3 shows the SEM images of the membrane surfaces. Both XLE and NF90 had rough surfaces with ridge-and-valley structures. FTIR results (Figure 4.4A) reveal their fully aromatic polyamide chemistry, with characteristic absorption bands of $1659 \mathrm{~cm}^{-1}$ (amide I band), $1611 \mathrm{~cm}^{-1}$ (aromatic amide band) and $1547 \mathrm{~cm}^{-1}$ (amide II band) (Tang et al., 2009a). The semi-aromatic polyamide NF270, indicated by an absence of the aromatic amide band and amide II band (Tang et al., 2009a), had a relatively smooth surface. SB50 was a cellulose acetate membrane with a smooth surface and was characterized by intense absorption bands at $1368 \mathrm{~cm}^{-1}(-\mathrm{OH}$ bending vibration) and $1034 \mathrm{~cm}^{-1}$ (C-O-C ether linkage from the glycosidic units) (Kamal et al., 2014). 


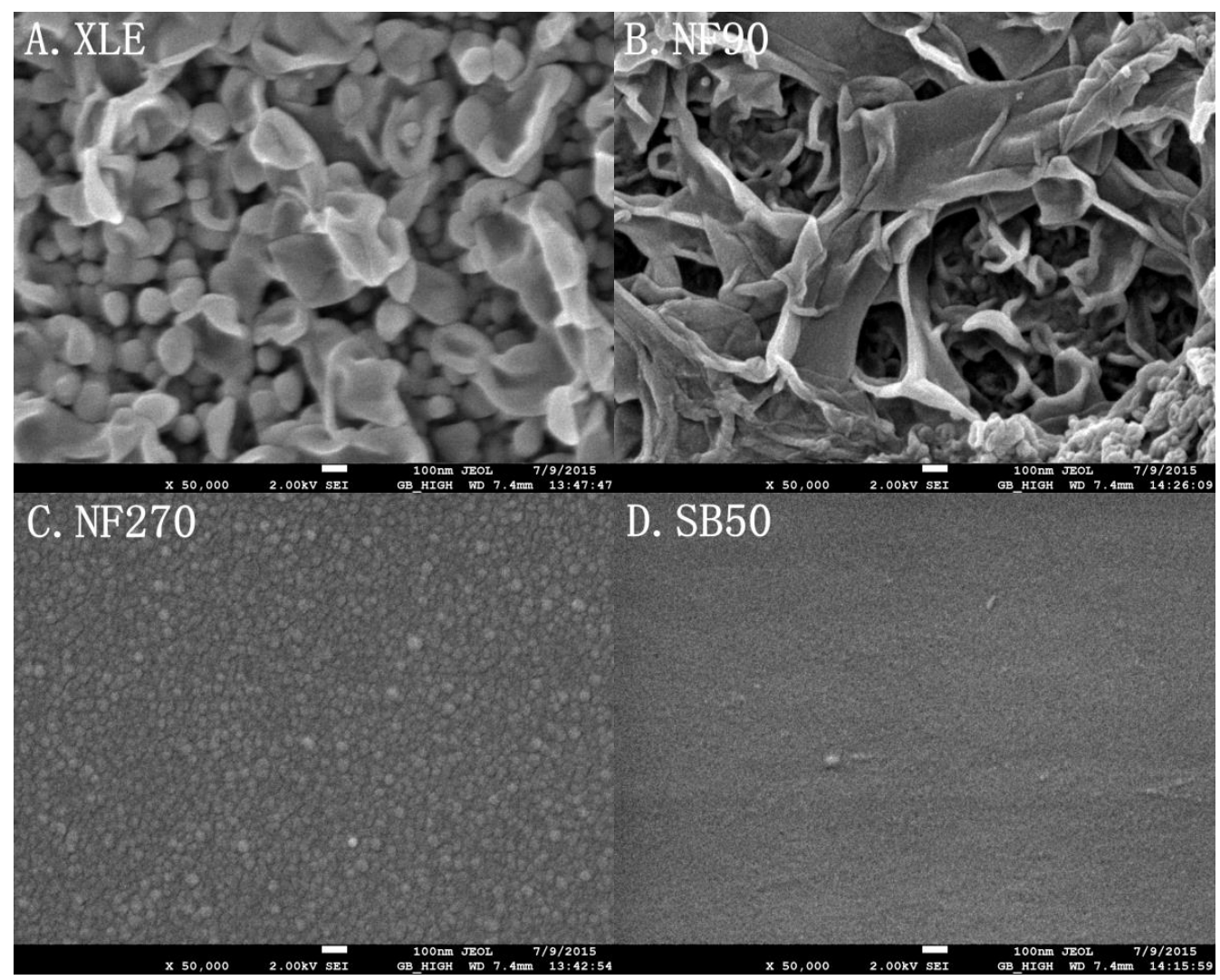

Figure 4.3 SEM images of the membrane rejection layers. Micrographs were obtained at an accelerating voltage of $2 \mathrm{keV}$. The scale bar is $100 \mathrm{~nm}$.

Zeta potential of the four membranes was measured in a $0.05 \mathrm{M} \mathrm{NaCl}$ solution over a $\mathrm{pH}$ range of $\sim 2-11$ (Figure 4.4B). Overall, zeta potential increased from negative to positive with the decreasing $\mathrm{pH}$. Compared to the cellulose acetate membrane SB50, the polyamide membranes appeared to be more charged, which can be explained by their amine and carboxylic functional groups (Tang et al., 2006). The isoelectric points were between 2.5-3.5 for XLE, NF270 and SB50, and slightly higher at $\sim 4.0$ for NF90, comparable to the literature (Do et al., 2012b). At pH 7.5 (representing the typical SPW pH range of 7.2-7.8), the zeta potentials were -33, 33, -36 and $-13 \mathrm{mV}$ for XLE, NF90, NF270 and SB50, respectively. 

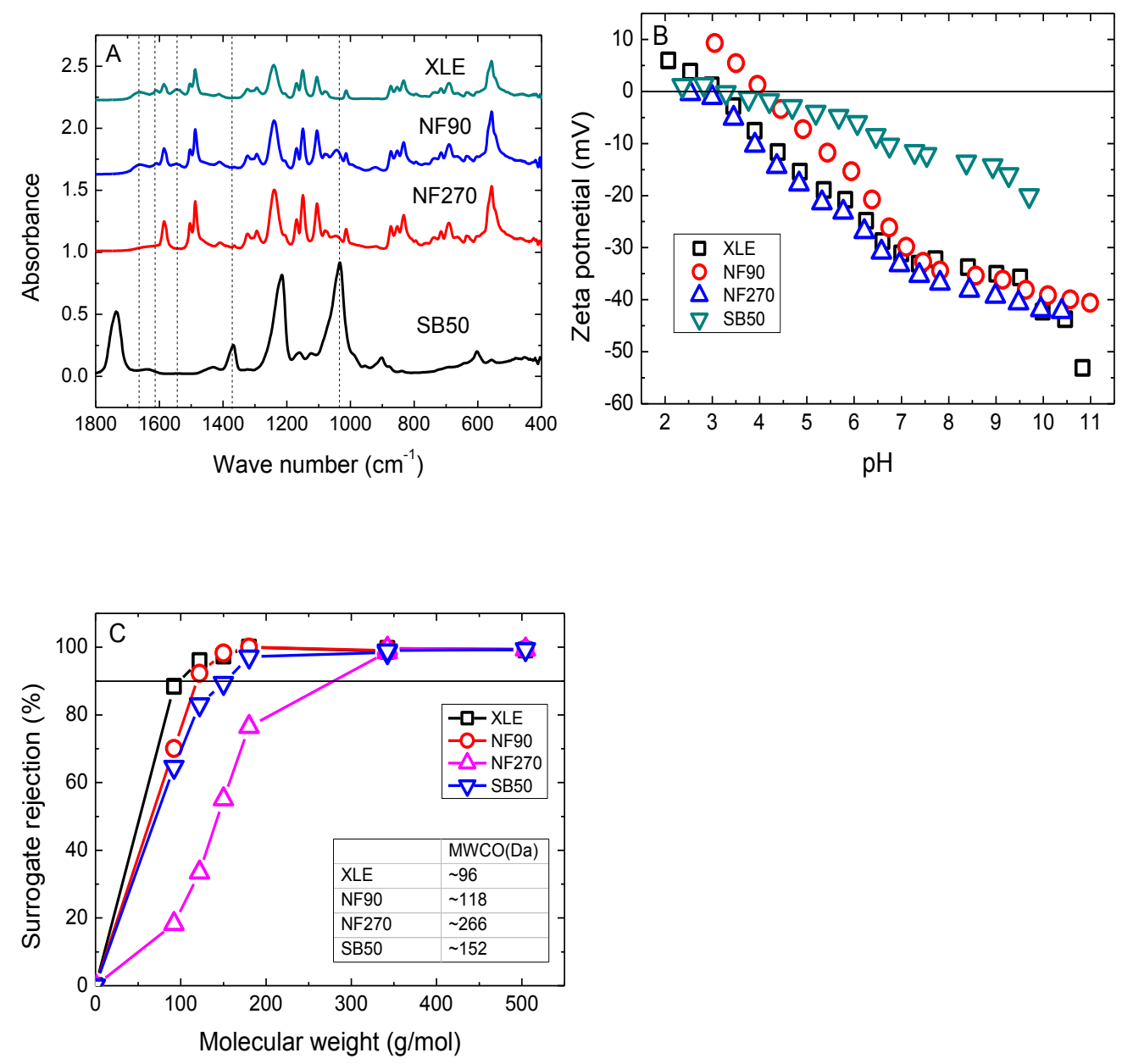

Figure 4.4 FTIR, zeta potential and MWCO of the four membranes. A: FTIR spectra of virgin membranes. B: Zeta potential of virgin membranes (in a $0.05 \mathrm{M} \mathrm{NaCl}$ background electrolyte over $\mathrm{pH} \sim 2-11)$. C: MWCOs of the membranes obtained from the surrogate rejection tests (experimental conditions: $100 \mathrm{psi}, \mathrm{pH} \sim 6.7,25^{\circ} \mathrm{C}$, feed water containing $200 \mathrm{mg} / \mathrm{L}$ of each surrogate).

The MWCOs, evaluated based on the rejections of neutral hydrophilic surrogates, were determined to be 96, 118, 266 and 152 Da for XLE, NF90, NF270 and SB50, respectively (Figure 4.4C). The reported MWCOs for NF90 and NF270 were 180 and 340 Da by López-Muñoz et al. (2009) and 200 and 300 Da by Do et al. (2012a) The difference of MWCOs could be explained by different steric characteristics of solutes, i.e., chain for polyethylene glycols (PEGs) and circular for 
most surrogates. Corresponding to its highest MWCO value, NF270 had the lowest $\mathrm{NaCl}$ rejection $(30.6 \%)$ and the largest membrane pore radius $(0.44 \mathrm{~nm})$.

\subsubsection{Rejection of HAAs}

\subsubsection{Effect of size exclusion}

Rejection by RO/NF can be affected by both size exclusion and electrostatic interaction (Nghiem et al., 2004). The effect of adsorption could be neglected due to the hydrophilic properties of HAAs and surrogate molecules. In order to isolate the effect of size exclusion, we performed some HAA rejection tests at $\mathrm{pH} 3.5$. At this $\mathrm{pH}$, the membranes were uncharged or only weakly charged such that the electrostatic interaction was negligible. Figure 4.5 presents the effect of molecular weight on the rejections of both HAAs and surrogate molecules by the four membranes, with a vertical dash line representing the MWCO of each membrane. A general trend of increased rejection at the higher molecular weight was observed, which confirms the critical role of size exclusion. However, the rejection data of HAAs had some significant scattering. More importantly, the rejection behavior of HAAs deviated apparently from that of the surrogate molecules. For molecules with similar molecular weights, HAAs had consistently lower rejections compared to the surrogates. The difference between the two groups was most significant for NF270 (Figure 4.5C), which was also the membrane having the largest pore radius of 0.44 nm. Indeed, the MWCO concept was inadequate to explain the low rejection values of $\sim$ or $<40 \%$ for those HAAs having molecular weights around or even greater than the MWCO of NF270 (266 Da). 

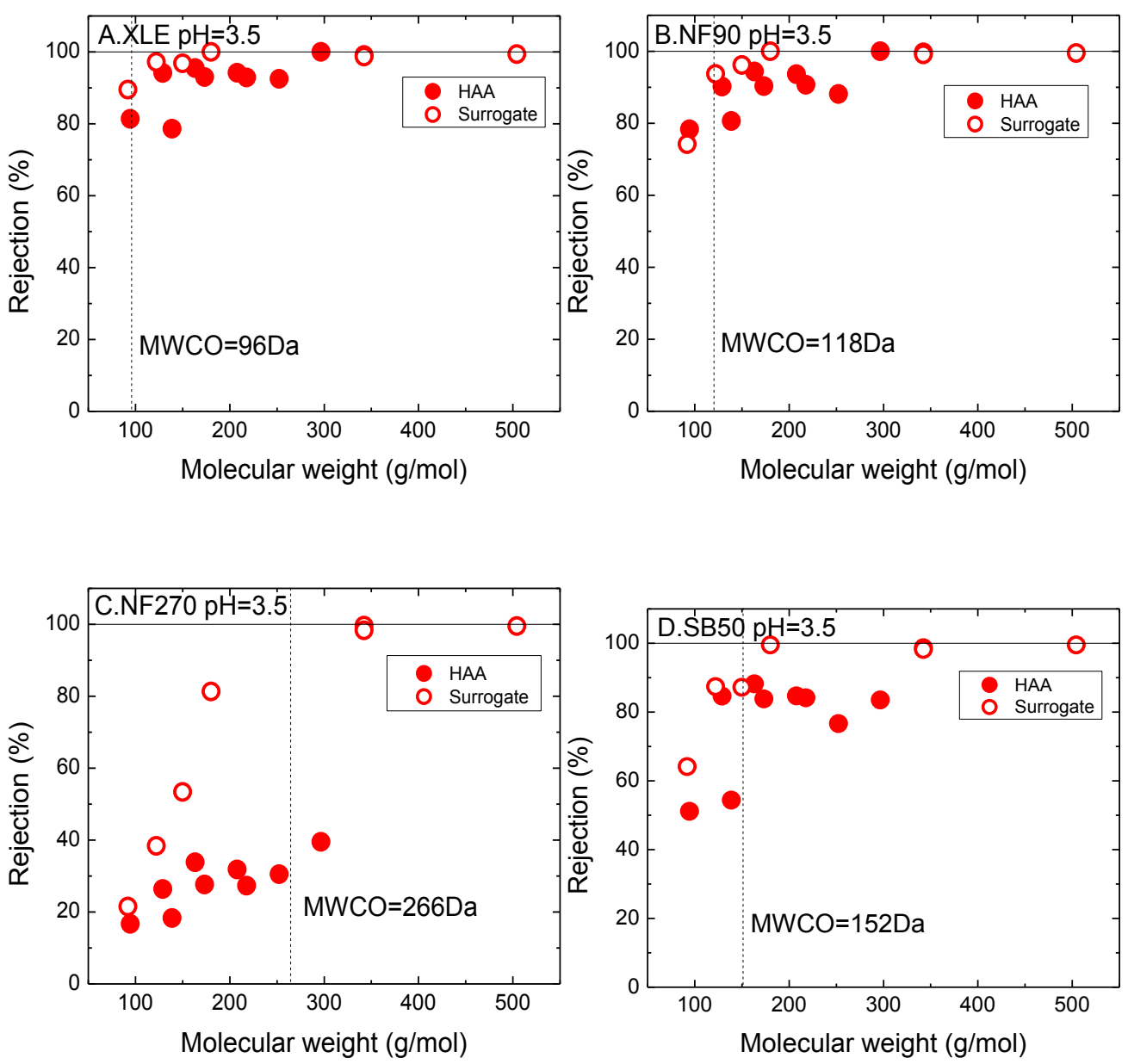

Figure 4.5 Effect of the molecular weight on rejection. Experimental conditions: 100 psi, pH $3.5,25^{\circ} \mathrm{C}$, feed water containing $100 \mu \mathrm{g} / \mathrm{L}$ of each HAA and $0.05 \mathrm{M} \mathrm{NaCl}$ for HAA rejection tests or $200 \mathrm{mg} / \mathrm{L}$ of each surrogate for surrogate rejection tests. Vertical dash lines represent the membrane MWCOs.

The discrepancy in rejection performance between HAAs and the surrogate molecules can be attributed to their difference in molecular structure (Table 4.1). The HAAs have a basic structure of acetic acid, with some hydrogen atoms substituted by halogens. In contrast, a number of the sugar-based surrogate molecules have circular structures, making them bulkier compared to the chain-like HAAs. For example, the Stokes radius of glucose (MW $=180 \mathrm{~g} / \mathrm{mol}$ ) is approximately $0.323 \mathrm{~nm}$, which is significantly larger than the sizes of HAAs with similar or even greater 
molecular weights. The current study suggests that the molecular weight, despite being the most commonly used parameter for modeling size exclusion (Chen et al., 2004; Ozaki and Li, 2002; Yangali-Quintanilla et al., 2010), is inadequate due to its inability to capture the molecular structure.

Figure 4.6 plots the rejections of HAAs and surrogates as a function of their molecular radii. Where applicable, the rejections of linear PEG molecules obtained from Do et al. (2012a), have been included for comparison purpose. Figure 4.6 shows a clear and consistent trend of increased rejections for molecules with greater molecular radii. For solutes with their radii bigger than the membrane pore size $\left(r_{s}>\right.$ $\left.r_{p}\right)$, both the HAAs and surrogates were highly rejected $(R>98 \%)$. The small percentage of the solutes passing through the membranes can be explained by a distribution of pore sizes and shapes in the membranes (Guillen and Hoek, 2010). The rejection decreased significantly for molecules with smaller molecular radii. The collapse of data points of both HAAs and surrogate molecules into a single trend line in Figure 4.6 reveals a more fundamental role of molecular radius in comparison to molecular weight in governing the solute transport through membranes. Since molecules with similar molecular weights can have drastically different molecular radii and thus rejection behavior, future studies shall consider the use of the latter as a preferred indicator for the description of size exclusion effect. 

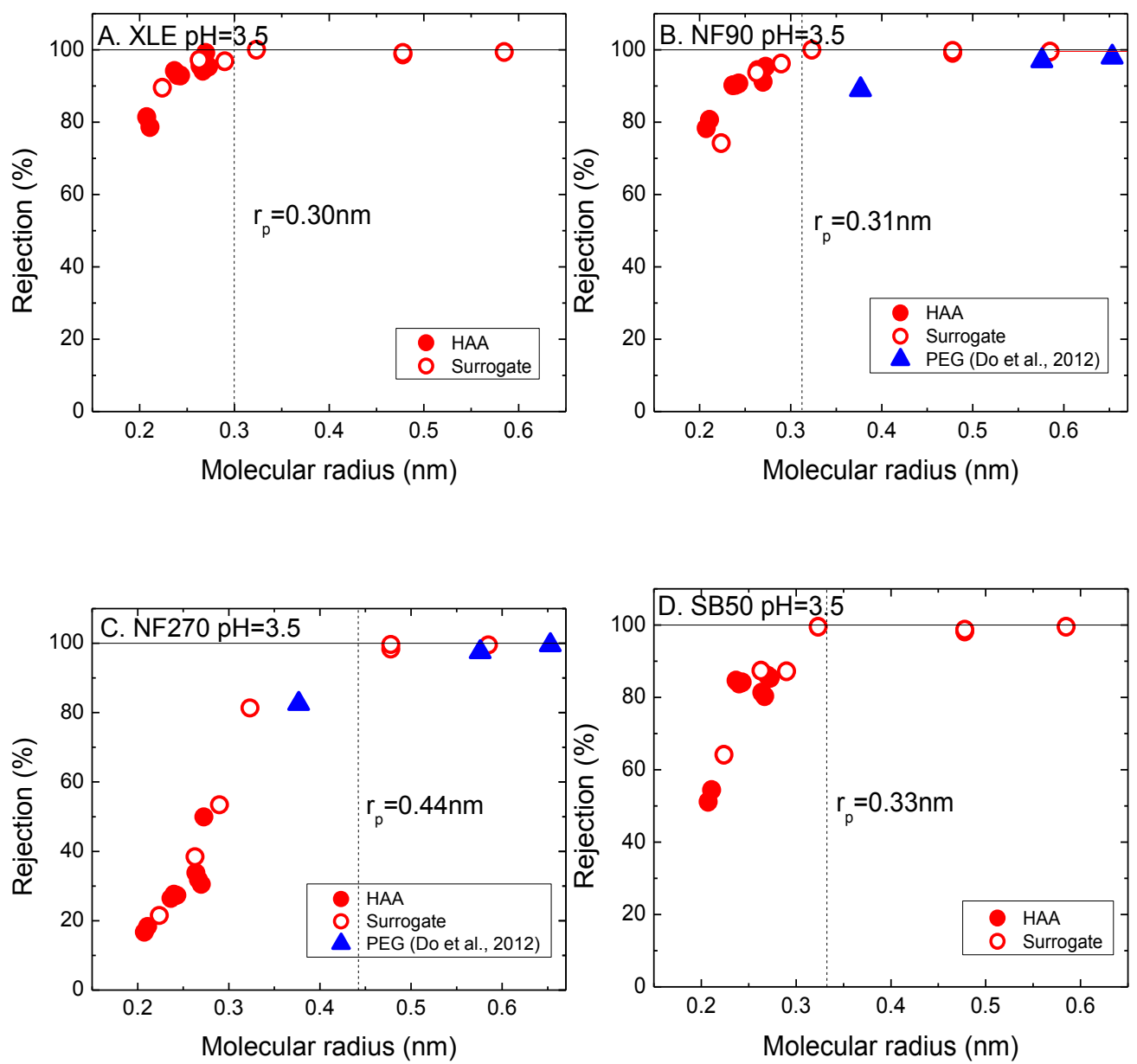

Figure 4.6 Effect of the molecular radius on rejection. Experimental conditions: 100 psi, pH $3.5,25^{\circ} \mathrm{C}$, feed water containing $100 \mu \mathrm{g} / \mathrm{L}$ of each HAA and $0.05 \mathrm{M} \mathrm{NaCl}$ for HAA rejection tests or $200 \mathrm{mg} / \mathrm{L}$ of each surrogate for surrogate rejection tests. Vertical dash lines represent the membrane pore radii.

\subsubsection{Effect of electrostatic interaction}

To investigate the effect of electrostatic interaction, solute rejections at different $\mathrm{pHs}$ were compared. Solution $\mathrm{pHs}$ had a negligible effect on the rejection of the neutral surrogate molecules (see Figure 4.7 for NF270 and Figure 4.8 for other membranes). This $\mathrm{pH}$-independent rejection behavior can be attributed to the lack of solutemembrane electrostatic interaction. The trend line in Figure 4.7A, which fits the 
surrogate data well at all $\mathrm{pHs,}$, provides a useful baseline for size exclusion (SE baseline).

Figure 4.7B presents the rejection of HAAs by NF270 at different pHs. To resolve the relative importance of size exclusion and charge interaction, the SE baseline obtained for the surrogates (Figure 4.7) is also shown. The rejection data of HAAs at $\mathrm{pH} 3.5$ and 5.5 falls nicely onto this baseline, suggesting that their rejection behavior was dominated by the size exclusion effect. At these pHs, most of HAAs $(\sim 85-100 \%)$ were dissociated to their anion forms. Nevertheless, NF270 was noncharged or weakly charged at $\mathrm{pH} 3.5$ and 5.5 (Figure 4.4B), which explains such dominance of size exclusion over charge interaction at these $\mathrm{pHs}$. The fitting by an identical baseline in Figure 4.7A and Figure 4.7B reveals that the effect of size exclusion may be adequately described by the molecular radius regardless of the detailed molecular structure.
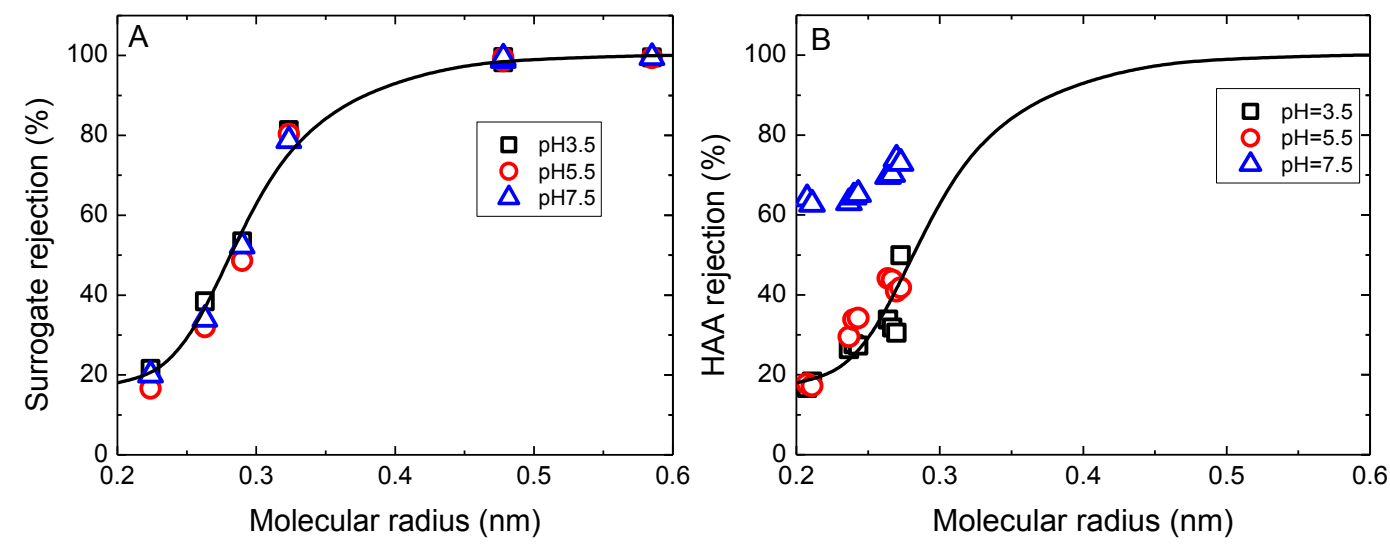

Figure 4.7 Effect of $\mathrm{pH}$ on rejections of surrogates (A) and HAAs (B) for NF270 membrane. Experimental conditions: $100 \mathrm{psi}$, pH over 3.5 to $7.5,25{ }^{\circ} \mathrm{C}$, feed water containing $100 \mu \mathrm{g} / \mathrm{L}$ of each $\mathrm{HAA}$ and $0.05 \mathrm{M} \mathrm{NaCl}$ for HAA rejection tests or 200 $\mathrm{mg} / \mathrm{L}$ of each surrogate for surrogate rejection tests. 

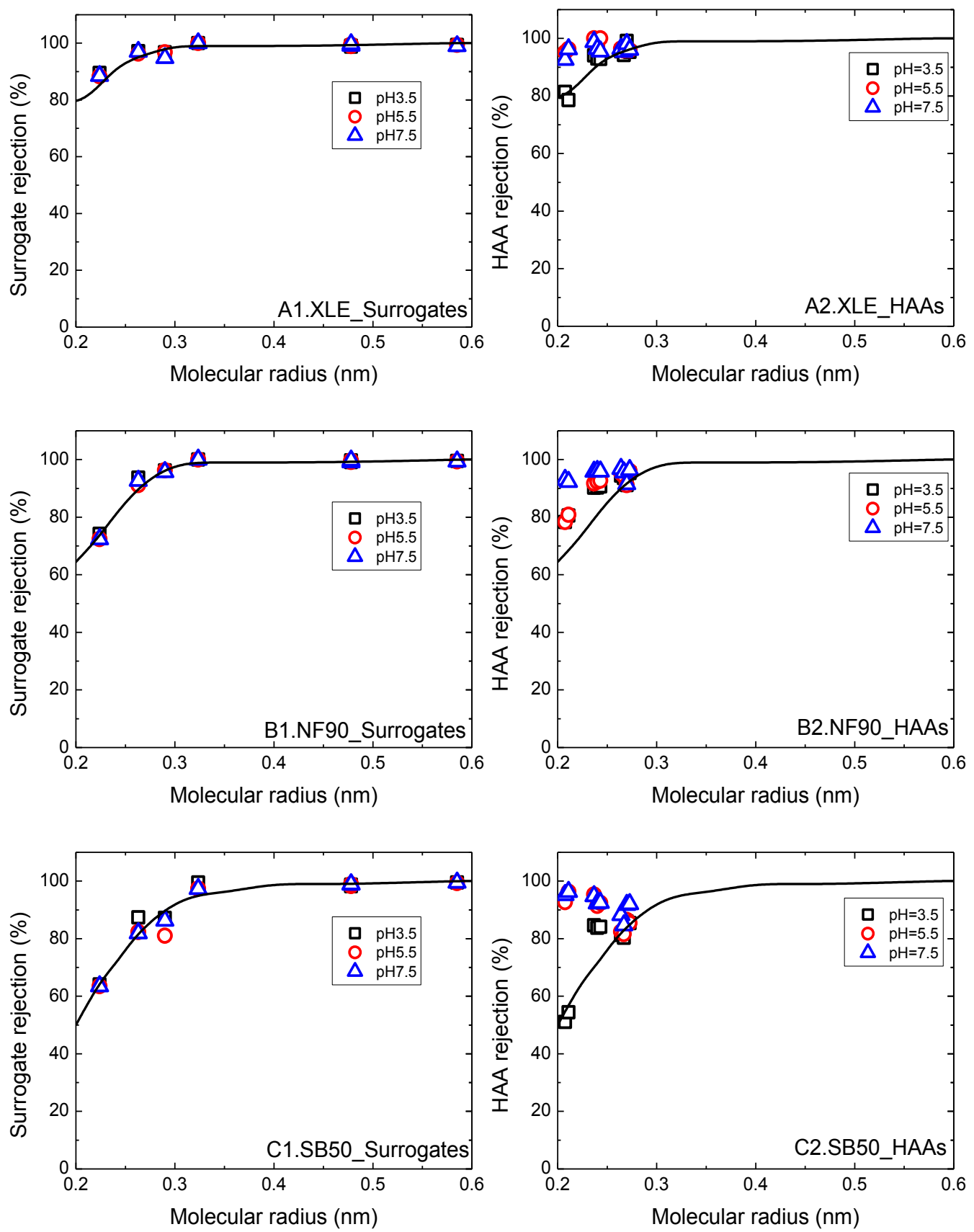

Figure 4.8 Effect of $\mathrm{pH}$ on rejections of surrogates and HAAs for XLE, NF90 and SB50 membranes. Experimental conditions: 100 psi, pH over 3.5 to $7.5,25^{\circ} \mathrm{C}$, feed water containing $100 \mu \mathrm{g} / \mathrm{L}$ of each HAA and $0.05 \mathrm{M} \mathrm{NaCl}$ for HAA rejection tests or $200 \mathrm{mg} / \mathrm{L}$ of each surrogate for surrogate rejection tests. 
Increasing $\mathrm{pH}$ from 3.5 to 7.5 significantly enhanced the rejection of HAAs by NF270 (> 60\%, see Figure 4.7B). At pH 7.5, the membrane became more negatively charged (zeta potential $\sim-36 \mathrm{mV}$, see Table 4.2), resulting in enhanced charge repulsion between the membrane and HAA anions (almost completely dissociated based on the pKa values (Bhattacharyya and Rohrer, 2012; Kong et al., 2014) in a range of $0.05-2.73$, see Table 4.1). The contribution of charge repulsion to solute rejection is represented by the vertical distance of the rejection data to the SE baseline. Figure 4.7B reveals several important trends with regard to the effect of electrostatic interaction on solute rejection: (1) solute rejection was greatly enhanced in the presence of strong charge repulsion; (2) charge repulsion played a more critical role for molecules with smaller molecular radii, i.e., molecules experiencing relatively weaker size exclusion effect; (3) the rejection of charged molecules had a much weaker dependence on the molecular radius (represented by a smaller slope) as a result of combined effects of charge interaction and size exclusion. The rejection enhancement due to charge repulsion was less significant for XLE, NF90, and SB50 (Figure 4.8). These membranes had much smaller pore sizes $(0.30-0.33 \mathrm{~nm})$ compared to the loose NF270 (pore radius $=0.44 \mathrm{~nm}$ ), implying a more important role of size exclusion over charge repulsion for these tight membranes. Nevertheless, the role of charge repulsion for these membranes was still non-trivial, resulting in consistently high rejections of $\sim$ or $>90 \%$ that would be otherwise difficult to achieve for the smaller molecules on the basis of size exclusion alone. 


\subsubsection{A unified approach for assessing size exclusion and charge repulsion}

Figure 4.7 demonstrates the feasibility of an SE baseline for resolving the role of size exclusion and charge repulsion on solute rejection by NF270. However, each membrane would require a separate SE baseline as a result of its different pore size (e.g., see Figure 4.8). Since the effect of size exclusion is ultimately determined by the size of the solute $\left(r_{s}\right)$ relative to the membrane pore size $\left(r_{p}\right)$, we attempted to use a normalized molecular size $\left(\lambda=r_{s} / r_{p}\right)$ to assess the importance of size exclusion. Figure 4.9A shows the rejection for both HAAs and surrogates by various membranes at a solution $\mathrm{pH}$ of 3.5. At this $\mathrm{pH}$, size exclusion would be the dominant rejection mechanism due to the absence of charge repulsion. In Figure 4.9A, solutes with radii bigger than the pore size $(\lambda>1)$ were nearly completely rejected $(R=\sim$ $100 \%)$. For smaller solutes $(\lambda<1)$, the rejection decreased significantly at reduced $\lambda$. In addition, all the data points can be approximately fitted by an identical trend line (the solid curve in Figure 4.9A) regardless of the membranes or solutes used, indicating $\lambda$ as the single most important parameter affecting size exclusion. Its invariance with respect to the properties of membranes and solutes may further allow the trend line in Figure 4.9A to be used as a unified baseline for evaluating size exclusion. 

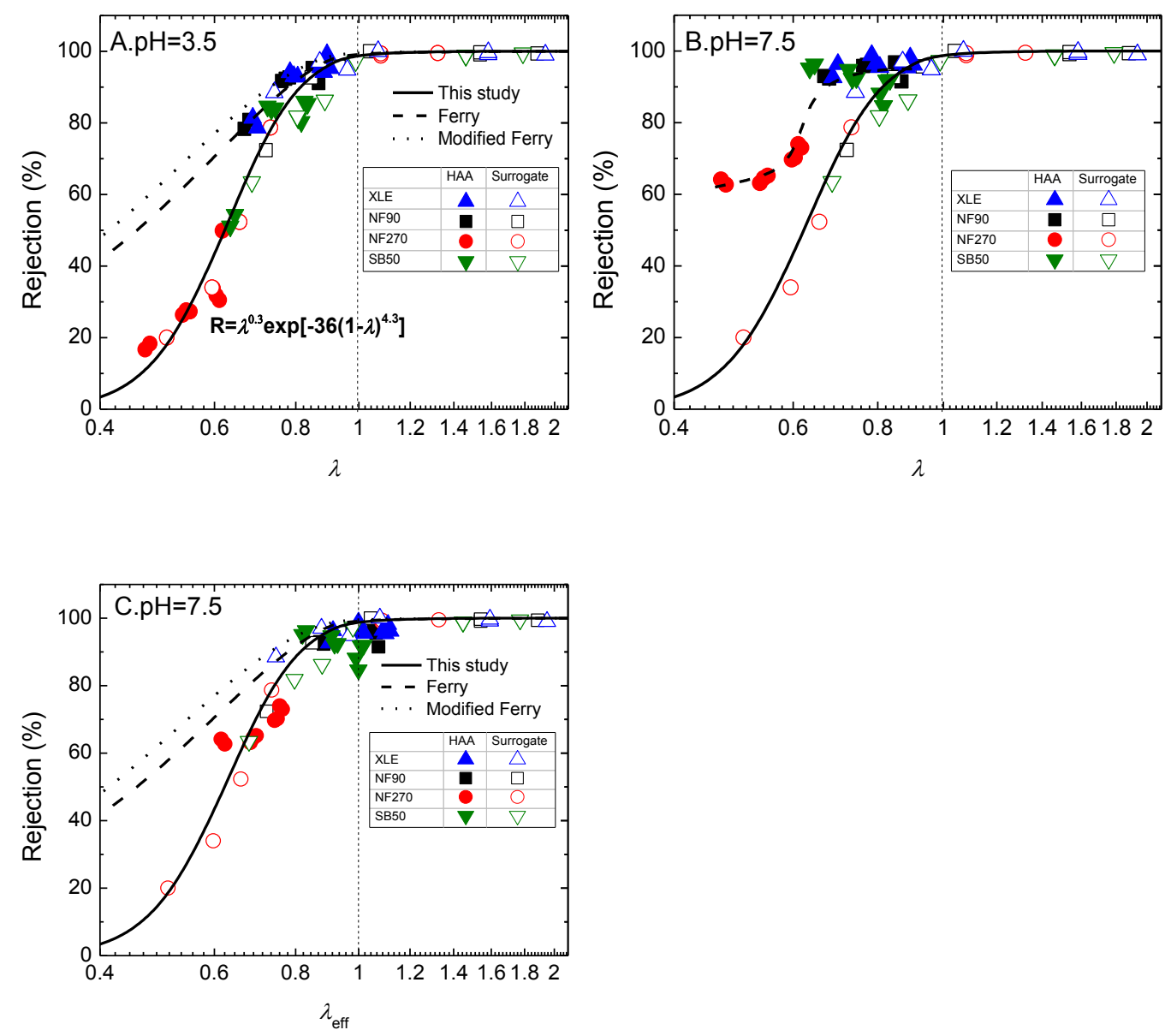

Figure 4.9 Normalized rejection evaluation. A. Normalized rejection as a function of $\lambda$ at $\mathrm{pH}$ 3.5. B. Normalized rejection as a function of $\lambda$ at $\mathrm{pH}$ 7.5. C. Normalized rejection as a function of $\lambda_{\text {eff }}$ at $\mathrm{pH}$ 7.5. Vertical dash lines represent a critical point where $\lambda$ or $\lambda_{\text {eff }}=1$. The effective hindrance parameter $\lambda_{\text {eff }}\left(=r_{\text {eff }} / r_{p}\right)$ is calculated based on an effective molecular radius $r_{\text {eff }}$ given by $r_{s}+0.045 \cdot \Lambda_{d}$.

Figure 4.9B presents the rejection of HAAs and surrogates at $\mathrm{pH}$ 7.5. Compared to the surrogates, HAAs had significantly enhanced rejection with respect to the SE baseline. The relatively high rejection of HAAs $(\sim$ or $>90 \%)$ was achieved with the presence of strong electrostatic repulsion for $\lambda>0.7$. However, such high rejection could not be maintained at smaller $\lambda$ values. Conceptually, the combined effect of electrostatic repulsion and size exclusion for HAAs may be captured by defining an effective molecular size $r_{\text {eff }}=r_{s}+k \cdot \Lambda_{d}$, with the Stokes radius $r_{s}$ accounting for size 
exclusion and the Debye length $\Lambda_{d}$ for electrostatic interaction $\left(\Lambda_{d}=1.4 \mathrm{~nm}\right.$ at an ionic strength of $50 \mathrm{mM}$ (Heimburg, 2008)), where $k$ is a dimensionless fitting coefficient. It should be noted that ionic strength may affect membrane surface charge and/or solute charge density, thereafter alter the electrostatic interaction between membranes and solutes (Mo et al., 2008; Oo and Song, 2009; Tang et al., 2007; Tu et al., 2011). Correspondingly, the change of ionic strength affects Debye length and therefore alters the effective molecular size and finally solute rejection. Figure 4.9C plots the HAA rejections as a function of the effective molecular size. By adopting a $k$ value of 0.045 , the HAA rejection data can be well fitted by the same baseline (i.e., the solid curves in Figure 4.9A,C). Thus, the concept of effective molecular size may potentially allow a unified approach to account for the effects of both size exclusion and charge repulsion. Nevertheless, further studies are required to relate the constant $k$ to solute and membrane properties (e.g., valence of the solutes and charge density of membranes).

\subsubsection{Implications}

The current study investigated the rejection of 9 HAAs and 7 surrogates by RO and NF membranes. Molecular radius was identified as a preferred parameter over molecular weight to capture the effect of size exclusion. In existing literature, the influence of size exclusion on rejection is often modeled by the Ferry's equation (Eq. (4.4)) (Ferry, 1936) or its modified version (Werber et al., 2016) (that takes account of the additional friction hindrance between solutes and pore walls, Eq. (4.5)): 
Ferry's model: $\quad R=\left\{\begin{array}{cc}{[\lambda(2-\lambda)]^{2}} & \lambda \leq 1 \\ 1 & \lambda>1\end{array}\right.$

Modified Ferry's model: $R=\left\{\begin{array}{cc}1-\left\{1-[\lambda(2-\lambda)]^{2}\right\} \exp \left(-0.7146 \lambda^{2}\right) & \lambda \leq 1 \\ 1 & \lambda>1\end{array}\right.$

These equations have been included in Figure 4.9 for comparison purpose. The current study shows that both the Ferry' model and its modified version overestimate the solute rejection by $\mathrm{RO}$ and $\mathrm{NF}$ membranes, particularly for $\lambda \sim$ or $<0.7$. Therefore, the application of the Ferry's model for RO and NF membranes requires further verification, noting that this model is derived based on continuum fluid mechanics (Ferry, 1936). On the basis of two boundary conditions $(\lambda=0, R=0 ; \lambda=$ $1, R=1$ ) and the curve fitting the rejection results (nonlinear least square best-fit method), we propose the following empirical equation:

$$
R=\left\{\begin{array}{cc}
\lambda^{0.3} \exp \left[-36(1-\lambda)^{4.3}\right] & \lambda \leq 1 \\
1 & \lambda>1
\end{array}\right.
$$

Eq. (4.6) is applicable to both HAAs and surrogates for the four RO/NF membranes, where differences in molecular structures and membrane separation properties are taken care by the single hindrance parameter $\lambda$. Furthermore, the effect of electrostatic interaction can also be accounted for by using $r_{\text {eff }}\left(=r_{s}+k \cdot \Lambda_{d}\right)$ for the calculation of the effective hindrance parameter $\lambda_{\text {eff }}\left(=r_{\text {eff }} t r_{p}\right)$. This approach provides a simple and rational way to treat the combined effects of size exclusion and 
electrostatic interaction. Future studies are required to validate its application to a wider variety of trace contaminants.

\subsection{Conclusions}

In this study, we investigated the removal of 9 HAAs by four commercial RO/NF membranes. To resolve the rejection mechanism, 7 neutral hydrophilic surrogates as molecular probes were used. The following conclusions can be drawn:

1) HAA rejections were $>60 \%$ for loose NF270 membrane and $\sim$ or $>90 \%$ for tighter membranes (XLE, NF90 and SB50) under typical SPW conditions (pH 7.5 and $50 \mathrm{mM}$ ionic strength).

2) The hindrance parameter, i.e., the ratio of molecular radius over membrane pore radius was a better SE descriptor compared to molecular weight when electrostatic interaction (e.g., pH 3.5) was negligible.

3) An effective molecular size of the solutes considering both charge repulsion and size exclusion could be a valid indicator of rejection.

4) The empirical formula derived may provide a simple and rational way for pool managers to have a primary screening of membrane selection to achieve the targeted rejection for contaminants of interest.

This fundamental mechanistic understanding on solute-membrane interaction would not be limited to the HAAs in SPWs, but rather should be applicable to HAAs or other contaminants in other types of water, e.g., DW, wastewater, etc. Nevertheless, the real application of contaminant removal by membrane technology should take the complex matrix effect into consideration. For example, we investigated the effect 
of calcium ions (which is ubiquitously present in SPWs but ignored in this chapter) on HAA removal in Chapter 5. In addition, the understanding of other matrix effects, e.g. humic acids, a variety of metal ions, etc., on the membrane performance will be needed as well. 


\section{Chapter 5 Role of Calcium Ions on the Removal of Haloacetic}

\section{Acids from Swimming Pool Water by Nanofiltration: Mechanisms and Implications}

\subsection{Introduction}

HAAs exist widely in a variety of water environments, e.g. wastewater, DWs, ground water and SPW, mainly attributed to the use of chlorination for water disinfection. As a group of known DBPs, HAAs have raised public concerns due to their potential genotoxicity and carcinogenicity (Richardson et al., 2007). EPA therefore has regulated a MCL of $60 \mu \mathrm{g} / \mathrm{L}$ for the sum of five HAAs for DWs (EPA, 1998). Our recent survey in Section 3.3.1 shows that HAA concentrations in typical SPWs reach more than an order of magnitude higher than the MCL. Other researchers have also reported high HAA concentrations in pools in the U.S. and Germany (Stottmeister and Naglitsch, 1996; Wang et al., 2014). Therefore, the potential health problem becomes a big challenge for swimmers exposed in SPWs with high HAAs.

Membrane technology has been used as an effective method for the removal of trace contaminants, e.g. DBPs, pharmaceuticals, pesticides, and hormones (Doederer et al., 2014; Nghiem et al., 2004; 2005; Simon et al., 2013). Good rejection of HAAs has been achieved ( $\geq 90 \%$ ) by either NF, RO, or FO membranes in the context of DWs and wastewater treatment (Chalatip et al., 2009; Kimura et al., 2003; Kong et al., 2014). In the previous chapter, we reported the use of NF/RO for the removal of HAAs under simulated SPW conditions (50 mM NaCl, $\mathrm{pH} 7.5)$ and demonstrated 
that the rejection was attributed to combined effects of size exclusion and charge repulsion. Nevertheless, real SPWs can be far more complicated and the matrix effect on the rejection of HAAs has yet to be systematically studied. In particular, calcium ions present commonly in SPWs as a second most abundant cation only after sodium ions, with a typical concentration of $\sim 0.5 \mathrm{mM}$ in Singapore pools and up to $\sim 2 \mathrm{mM}$ in other pools (Buczkowska-Radlinska et al., 2013). Compared to other common ions (e.g., $\mathrm{Na}^{+}, \mathrm{K}^{+}, \mathrm{Mg}^{2+}, \mathrm{Cl}^{-}$) in $\mathrm{SPWs}, \mathrm{Ca}^{2+}$ possesses a greater potential to affect membrane performance, attributing to its stronger ability to interact with a variety of solutes as well as membrane materials (Ahn et al., 2008). In the context of membrane fouling, the role of $\mathrm{Ca}^{2+}$ has been well documented (Tang et al., 2011). $\mathrm{Ca}^{2+}$ can form complexes with certain ligand groups (e.g., - $\mathrm{COO}^{-}$) of proteins, polysaccharides, and natural organic matters, leading to charge neutralization and bridging effects with typically increased fouling tendency (Ang and Elimelech, 2008; Hong and Elimelech, 1997; Tang et al., 2007; van den Brink et al., 2009; Yoon et al., 1998). It has also been reported that $\mathrm{Ca}^{2+}$ can significantly alter the surface properties of polyamide membranes by binding to the carboxylic groups contained in these membranes (Herzberg et al., 2009; Jin et al., 2009; Mi and Elimelech, 2010; Motsa et al., 2014). On the other hand, only a handful of studies have systematically examined the effect of $\mathrm{Ca}^{2+}$ on trace contaminant removal by membranes (Mahlangu et al., 2014; Zhao et al., 2013).

The coexistence of HAAs and $\mathrm{Ca}^{2+}$ in typical SPW matrix arouses the necessity to explore the HAA removal from SPWs containing $\mathrm{Ca}^{2+}$, which has seldom been 
investigated. Presumably, $\mathrm{Ca}^{2+}$ can interact either with HAAs or membranes (e.g., by binding to the carboxylic groups to form $\mathrm{HAA}-\mathrm{Ca}^{2+}$ or membrane- $\mathrm{Ca}^{2+}$ complex) therefore altering the rejection behaviour. We were therefore prompted to investigate the effect of $\mathrm{Ca}^{2+}$ on HAA removal systematically. We analysed the rejection of 9 HAAs together with 7 surrogate molecules by two commercial NF membranes (NF90 and NF270) in order to resolve the underlying mechanisms involved with respect to the role of $\mathrm{Ca}^{2+}$ in trace contaminant rejection. The fundamental mechanistic understanding will provide deeper insights for further extending of the membrane technology for the SPW treatment.

\subsection{Materials and methods}

\subsubsection{Chemicals and materials}

HAAs. Nine HAAs as shown in Table 5.1 were used in this study. All of them were purchased from Sigma-Aldrich in analytical grades with $\geq 97 \%$ purity. A stock solution containing a mixture of $1 \mathrm{~g} / \mathrm{L}$ of each HAA was prepared by dissolving the pure chemicals in MilliQ water (Millipore, Billerica, MA). Reagents used for HAA quantification were of GC grades for solvents (e.g. MTBE and methanol) and of at least ACS grades for other chemicals (e.g. 98\% sulfuric acid, sodium bicarbonate, copper sulfate and sodium sulfate).

Surrogate compounds. Glycerol, erythritol, xylose, glucose, maltose, sucrose and raffinose were of analytical grades with purity over $99 \%$. Sucrose was purchased 
from USB and the others were from Sigma-Aldrich. These neutral hydrophilic compounds were used as additional probe molecules for resolving the mechanisms involved in solute rejection by membranes. A mixture of $40 \mathrm{~g} / \mathrm{L}$ of each surrogate molecule was prepared with MilliQ water as the stock solution.

Table 5.1 Properties of HAAs and surrogate molecules

\begin{tabular}{|c|c|c|c|c|c|c|}
\hline Name & Abbreviation & $\begin{array}{l}\text { Molecular } \\
\text { weight } \\
(\mathrm{g} / \mathrm{mol})\end{array}$ & $\begin{array}{l}\text { Stokes } \\
\text { radius }^{\mathrm{a}} \\
(\mathrm{nm})\end{array}$ & $\begin{array}{l}\text { Concentration } \\
(\mu \mathrm{M})\end{array}$ & $\mathrm{pK}_{\mathrm{a}}^{\mathrm{a}}$ & $\log \mathrm{K}_{1}^{\mathrm{b}}$ \\
\hline \multicolumn{7}{|c|}{ HAAs } \\
\hline Chloroacetic acid & MCAA & 94.5 & 0.207 & 1.0582 & 2.65 & 0.14 \\
\hline Bromoacetic acid & MBAA & 139.0 & 0.211 & 0.7197 & 2.73 & 0.55 \\
\hline Dichloroacetic acid & DCAA & 128.9 & 0.237 & 0.7756 & 1.37 & N.A. \\
\hline $\begin{array}{l}\text { Bromochloroacetic } \\
\text { acid }\end{array}$ & BCAA & 173.4 & 0.240 & 0.5767 & 1.39 & N.A. \\
\hline Dibromoacetic acid & DBAA & 217.8 & 0.243 & 0.4591 & 1.47 & N.A. \\
\hline Trichloroacetic acid & TCAA & 163.4 & 0.264 & 0.6120 & 0.09 & N.A. \\
\hline Tribromoacetic acid & TBAA & 296.8 & 0.273 & 0.3370 & 0.22 & N.A. \\
\hline $\begin{array}{l}\text { Dibromochloroacetic } \\
\text { acid }\end{array}$ & DBCAA & 252.3 & 0.270 & 0.3964 & 0.13 & N.A. \\
\hline $\begin{array}{l}\text { Bromodichloroacetic } \\
\text { acid }\end{array}$ & BDCAA & 207.8 & 0.267 & 0.4811 & 0.05 & N.A. \\
\hline Acetic acid & AA & $\begin{array}{l}60.1 \\
\text { Surrogates }\end{array}$ & -- & $1.0000^{c}$ & 4.75 & 1.18 \\
\hline Glycerol & & 92.1 & 0.224 & & 14.15 & \\
\hline Erythritol & & 122.1 & 0.263 & & 13.27 & \\
\hline Xylose & & 150.1 & 0.290 & & 12.15 & \\
\hline Glucose & & 180.2 & 0.323 & & 12.28 & \\
\hline Maltose & & 342.3 & 0.478 & & 11.94 & \\
\hline Sucrose & & 342.3 & 0.478 & & 12.62 & \\
\hline Raffinose & & 504.4 & 0.585 & & 12.74 & \\
\hline
\end{tabular}

Other chemicals. Sodium chloride and calcium chloride were with purity over $99 \%$ from Merck. Sodium hydroxide and hydrochloric acid (37\%) used for $\mathrm{pH}$ adjustment were at least analytical grade. Potassium bromide (Sigma, 99\%), lithium chloride 
(ACROS, anhydrous, 99\%), boric acid (ACS, ISO, Ph Eur, Merck, 99.5-100.5\%), sodium nitrate (Sigma, ACS, > 99.0\%), sodium sulfate (Sigma, granular, ACS, > 99\%) and urea (Ph Eur, ACS, Merck) were used for the preparation of synthetic SPW. Real SPWs were collected from a public outdoor pool on the campus of Nanyang Technological University, Singapore. The major criteria for the synthetic SPW are: 1) synthetic SPW had a similar ionic strength to the real SPW, and an intermediate $\mathrm{pH}$ value of 7.5 based on the regulated $\mathrm{pH}$ range of 7.20-7.80 for SPW context by NEA, Singapore; 2) major ions in the synthetic SPW had similar concentrations to those in the real SPW (with the exception that higher concentrations were adopted for components with high detection limits to facilitate our analysis, see Table 5.4); 3) constituents that may cause membrane scaling (e.g., Fe and Al) were omitted. A stock solution was first prepared in accordance to Table 5.2, and the synthetic SPW was prepared by a 100-fold dilution of the stock solution.

Table 5.2 Recipe for the preparation of synthetic SPW

\begin{tabular}{llll}
\hline Constituents & Source compounds $\begin{array}{l}\text { Stock solution } \\
(\mathrm{mg} / \mathrm{L})\end{array}$ & $\begin{array}{l}\text { Synthetic SPW } \\
(\mathrm{mg} / \mathrm{L})\end{array}$ \\
\hline $\mathrm{K}$ & $\mathrm{KBr}$ & 2000 & 20 \\
$\mathrm{Li}$ & $\mathrm{LiCl}$ & 10 & 0.1 \\
$\mathrm{~B}$ & $\mathrm{H}_{3} \mathrm{BO}_{3}$ & 10 & 0.1 \\
$\mathrm{NO}_{3}$ & $\mathrm{NaNO}_{3}$ & 2000 & 20 \\
$\mathrm{SO}_{4}$ & $\mathrm{Na}_{2} \mathrm{SO}_{4}$ & 6000 & 60 \\
$\mathrm{Br}$ & $\mathrm{KBr}_{\mathrm{Urea}}$ & 4103 & 41 \\
$\mathrm{Ca}$ & $\mathrm{Urea}$ & 1000 & 10 \\
\hline $\begin{array}{l}\text { Note: } \\
\text { a NaCl and calcium were not added during the preparation of the stock solution, however 50 mM }\end{array}$ \\
$\begin{array}{l}\text { NaCl and calcium in a concentration range of } 0-40 \text { mg/L were added to the synthetic SPW after the } \\
\text { dilution of the stock solution. }\end{array}$
\end{tabular}


NF membranes. Two commercial NF membranes were investigated in this study, including a fully aromatic polyamide membrane NF90 and a semi-aromatic polyamide membrane NF270 (Dow Filmtec) (Tang et al., 2009a; b). The physiochemical properties of these two membranes have been characterized in last chapter and are summarized in Table 5.3. Compared to the loose NF270, NF90 is a tighter nanofiltration membrane characterized with its higher $\mathrm{NaCl}$ rejection, smaller MWCO and pore radius, as well as lower water permeability. 
Table 5.3 Membrane properties (Data were extracted from Table 4.2, except RMS roughness from Tang et al. (2009b) and contact angle by this study)

\begin{tabular}{|c|c|c|c|c|c|c|c|c|c|}
\hline Membrane & $\begin{array}{l}\text { Surface layer } \\
\text { material }\end{array}$ & Manufacturer & $\begin{array}{l}\text { Water } \\
\text { permeability } \\
\mathrm{L} / \mathrm{m}^{2} \mathrm{~h} \text { bar }\end{array}$ & $\begin{array}{l}\text { Nominal } \\
\mathrm{NaCl} \\
\text { rejection }^{\mathrm{b}} \\
\%\end{array}$ & $\begin{array}{l}\mathrm{MWCO}^{\mathrm{c}} \\
\mathrm{Da}\end{array}$ & $\begin{array}{l}\zeta \text { potential at } \\
\mathrm{pH} 7.5^{\mathrm{d}} \\
\mathrm{mV}\end{array}$ & $\begin{array}{l}\text { Pore } \\
\text { radius }^{\mathrm{e}} \\
\mathrm{nm} \\
\end{array}$ & $\begin{array}{l}\mathrm{RMS} \\
\text { roughness } \\
\mathrm{nm} \\
\end{array}$ & $\begin{array}{l}\text { Contact } \\
\text { angle } \\
\text { o }\end{array}$ \\
\hline NF90 & $\begin{array}{l}\text { Fully aromatic } \\
\text { polyamide }\end{array}$ & Dow Filmtec & 7.69 & 82.7 & 118 & -33 & $\begin{array}{l}0.31 \\
\left(0.31^{f}\right)\end{array}$ & $142.8 \pm 9.6$ & $54.1 \pm 2.6$ \\
\hline NF270 & $\begin{array}{l}\text { Semi- aromatic } \\
\text { polyamide }\end{array}$ & Dow Filmtec & 16.10 & 30.6 & 266 & -36 & $\begin{array}{l}0.40 \\
\left(0.44^{f}\right)\end{array}$ & $9.0 \pm 4.2$ & $26.5 \pm 1.7$ \\
\hline
\end{tabular}

Note:

${ }^{a}$ Water permeability was obtained by compacting the membranes for $24 \mathrm{~h}$ under the defined conditions $\left(100 \mathrm{psi}, 25{ }^{\circ} \mathrm{C}, \mathrm{pH} \sim 6.7\right.$, MilliQ water).

${ }^{\mathrm{b}} \mathrm{NaCl}$ rejection was determined after $\mathrm{NaCl}$ was dosed to the feed tank for $24 \mathrm{~h}$ under defined conditions $\left(100 \mathrm{psi}, 25^{\circ} \mathrm{C}, \mathrm{pH} \sim 6.7,50 \mathrm{mM} \mathrm{NaCl}\right)$.

${ }^{\mathrm{c}}$ MWCOs were obtained using neutral hydrophilic compounds as the probing molecules.

${ }^{\mathrm{d}}$ Zeta potential was evaluated at $\mathrm{pH} 7.5$ with $50 \mathrm{mM} \mathrm{NaCl}$ as the background electrolyte.

e Pore radius was calculated by a solute transport model in accordance to Nghiem et al. (2004).

${ }^{\mathrm{f}}$ The calculated pore radius of NF270 was $0.40 \mathrm{~nm}$, which was slightly smaller compared to the value reported in our previous study ( 0.44 nm). The slight difference may arise from the different batches of membrane materials. The calculated pore radius of NF90 was identical in two studies. 


\subsubsection{Membrane characterization}

Zeta potential. The zeta potential of the membrane surface was measured by a SurPASS electrokinetic analyzer (Anton Paar GmbH, Graz, Austria). The channel height of $100-150 \mu \mathrm{m}$ was maintained between two adjustable gap cells $(20 \times 10$ $\mathrm{mm})$. Two pieces of virgin membranes were attached to each cell with face-to-face orientation. The $50 \mathrm{mM} \mathrm{NaCl}$ solution was used as a background electrolyte and the solution $\mathrm{pH}$ was manually adjusted to 7.5 by $1 \mathrm{M} \mathrm{NaOH}$. In order to assess the effect of $\mathrm{Ca}^{2+}$ on the membrane surface charge, a predetermined volume of a $0.1 \mathrm{M} \mathrm{CaCl}_{2}$ solution was manually pipetted to achieve a $\mathrm{Ca}^{2+}$ concentration over the range of 0 to $1 \mathrm{mM}$. Experiments showed that a 10-min stirring by an electromagnetic agitator was enough for solution stabilization. Then the measurement was conducted twice for the same membrane coupon, and totally 4 values were obtained for each condition. The average zeta potential was calculated based on the HS equation (Chun et al., 2003).

Contact angle. The contact angle of membranes was measured by a Goniometer (DataPhysics, OCA 15EC). The dried membrane samples were attached to a glass slide by a double-sided tape to ensure a flat membrane surface before measurement. The contact angle of each sample was measured for at least 10 times at different locations. The average and standard deviation were obtained by discarding the maximum and minimum values (Table 5.3). 
XPS analysis. The elemental composition of the material from the top 0 to $5 \mathrm{~nm}$ of the surface region can be determined by X-ray photoelectron spectroscopy (XPS, Thermo Scientific Escalab 250Xi) with a detection limit of $0.1 \%$. The $\mathrm{Ca}^{2+}$ binding to the membrane surface was quantified by XPS analysis, following the sample preparation procedures proposed by Do et al. (2012c). Briefly, the virgin membranes were thoroughly rinsed and soaked in the MilliQ water for $24 \mathrm{~h}$. They were then immersed in $\mathrm{CaCl}_{2}$ solutions (e.g., $0.1,1 \mathrm{mM}$, and $0 \mathrm{mM}$ as a control) with $\mathrm{pH}$ adjustment to 7.5 by $0.1 \mathrm{mM} \mathrm{HCl}$ or $\mathrm{NaOH}$ for $60 \mathrm{~min}$. The membranes were then

rinsed with $0.001 \mathrm{mM} \mathrm{CaCl}_{2}$ solution (at $\mathrm{pH} 7.5$ ) for 4 times to remove the $\mathrm{Ca}^{2+}$ that was not binded to the membrane surface. The membranes were dried in vacuum before XPS analysis.

\subsubsection{Membrane filtration experiments}

Membrane setup. A bench scale cross-flow membrane filtration setup was used in this study. The details for the setup and filtration protocol have been elaborated in last chapter and briefly described as follows (see Figure 4.1). The filtration system contains four parallel cross-flow CF042 cells (an effective area of $42 \mathrm{~cm}^{2}$ and a channel size of $4.6 \times 9.2 \mathrm{~cm}$, Delrin Acetal, Sterlitech, Kent, WA, USA). A spacer (GE Osmonics, Minnetonka, MN, USA) of $1.2 \mathrm{~mm}$ thickness was placed in each cell for the filtration tests. A chiller (Polyscience, Niles, IL, USA) was used to control the temperature of feed solution at $25^{\circ} \mathrm{C}$. 
Filtration experiments. Before the filtration tests, the virgin membranes (after being soaked for $24 \mathrm{~h}$ in the MilliQ water) were loaded into the cell. The permeate and retentate circulated back to the feed tank to maintain the feed concentration constant. For all filtration tests, the applied pressure, cross-flow and solution temperature were kept at $100 \mathrm{psi}, 2.4 \mathrm{~L} / \mathrm{min}$ (corresponding to $54.3 \mathrm{~cm} / \mathrm{s}$ cross-flow velocity) and $25^{\circ} \mathrm{C}$, respectively. To minimize the effect of membrane compaction, all membrane coupons were filtered for $24 \mathrm{~h}$ in the feed solutions before sample collection for analysis.

Rejection of ionic species was measured for both real and synthetic SPWs (Table 5.4). The HAA rejection tests ( $100 \mu \mathrm{g} / \mathrm{L}$ of each HAA in the feed) were conducted for synthetic SPW. The solution $\mathrm{pH}$ was adjusted to 7.5 by dosing $1 \mathrm{M} \mathrm{NaOH}$ or $\mathrm{HCl}$ and measured by a portable $\mathrm{pH}$ meter (Mettler Toledo, SevenGo pro). An additional solution $\mathrm{pH}$ of 3.5 for HAA rejection tests was also included for comparison purpose. To study the effect of $\mathrm{Ca}^{2+}, \mathrm{CaCl}_{2}$ stock solution $(0.1$ or $1 \mathrm{M})$ was added to the feeds to achieve a predetermined $\mathrm{Ca}^{2+}$ concentration over the range of 0 to $1 \mathrm{mM}$. The same membrane coupon was used for different $\mathrm{Ca}^{2+}$ concentrations to diminish the minor material difference of different regions. The filtration setup was run for $1 \mathrm{~h}$ at each $\mathrm{Ca}^{2+}$ concentration to ensure system stabilization, after which both feed and permeate were collected for analysis. 
Surrogate rejection tests were performed using a feed solution containing $200 \mathrm{mg} / \mathrm{L}$ of each surrogate at $\mathrm{pH}$ 7.5. Background electrolytes were not added in the surrogate rejection tests to avoid its interference with the surrogate analysis by HPLC-RID.

\subsubsection{Analytical methods}

HAA quantification. HAAs were quantified based on a modified EPA 552.3 method proposed in Section 3.2.1. This method involved three key steps: 1) liquidliquid extraction by solvent MTBE; 2) derivatization of HAAs by acidic methanol (10\% sulfuric acid in methanol); 3) analysis of methylated HAAs by GCMS (GC: Agilent 7890B; MS: Agilent 5977A; column: DB-5MS (J \& W Scientific, 5\% phenyl - 95\% dimethyl polysiloxane, $30 \mathrm{~m} \times 0.25 \mathrm{~mm}$ ID, $0.25 \mu \mathrm{m}$ film thickness)). The flow chart of HAA analysis procedure has been shown in Figure 3.1.

Surrogate quantification. HPLC-RID (Agilent 1260 Infinity) has been demonstrated as an effective technique to quantify these surrogates (Section 4.2.4). The columns used were Hi-Plex $\mathrm{Pb}(7.7 \times 300 \mathrm{~mm}, 8 \mu \mathrm{m}$, guard $) /(7.7 \times 50 \mathrm{~mm}, 8$ $\mu \mathrm{m}$, analytical). The detailed information for this analysis was described in Section 4.2.4.

Elemental quantification. The elements, including $\mathrm{Na}, \mathrm{Ca}, \mathrm{K}, \mathrm{Mg}, \mathrm{Fe}, \mathrm{Mn}, \mathrm{Cu}, \mathrm{Sr}$, $\mathrm{Zn}, \mathrm{Li}, \mathrm{Al}$, and $\mathrm{B}$, were measured by an inductively coupled plasma - optical emission spectrometry (ICP-OES, PerkinElmer, UK) for concentrations with the level of milligram per liter and/or by an inductively coupled plasma - mass 
spectroscopy (ICP-MS) for concentrations with the level of microgram per liter. The anions, including $\mathrm{F}, \mathrm{Cl}, \mathrm{NO}_{2}, \mathrm{NO}_{3}, \mathrm{SO}_{4}, \mathrm{PO}_{4}$, and $\mathrm{Br}$, were determined by an ion chromatography (IC, Dionex ICS-1100 with autosampler Dionex AS-HV). Ammonia ions were determined by chromogenic reactions based on the salicylate method detected by a portable DR 2800 spectrophotometer (Hach, USA). TOC and TN were measured by a TOC_N analyzer (multi N/C ${ }^{\circledR} 2100 S$, Analytikjena, Germany). Urea was determined based on the enzymatic and colorimetric indophenol blue method (Microquant ${ }^{\circledR}$ urea test; from Merck) by a UV/VisSpectrophotometer (Cary 50, Agilent) under a wavelength of $690 \mathrm{~nm}$ with a calibration range of $0.1-10 \mathrm{mg} / \mathrm{L}$ (Figure 5.1).
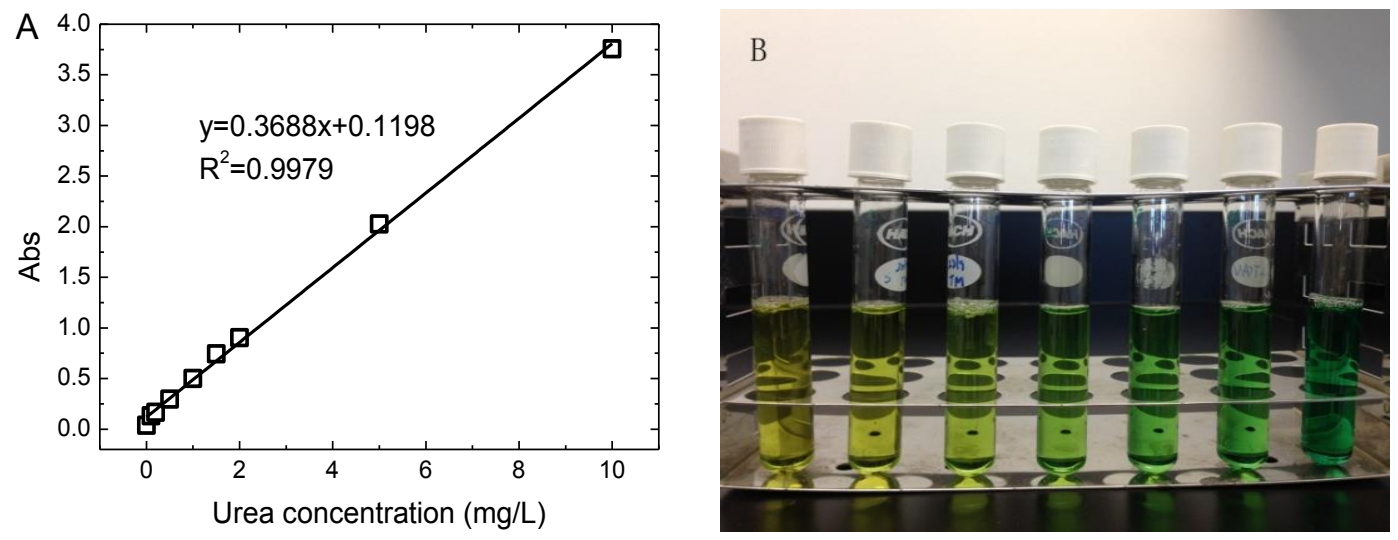

Figure 5.1 Urea calibration curve (A) and the calibration standard illustration (B)

\subsection{Results and discussion}

\subsubsection{Properties of real SPWs}

The characteristics of a typical real SPW are shown in Table 5.4. Sodium and chloride ions are two predominant species $(\sim 50 \mathrm{mM})$ in SPWs, which control the 
solution ionic strength and lead to the high conductivity $(\sim 5 \mathrm{~ms} / \mathrm{cm})$. The circulated SPW treatment system might cause the accumulation of the salinity, which contains two sources, body fluid (e.g., sweat and urine) released from the bathers and chemicals (e.g., $\mathrm{NaClO}$ for disinfection and $\mathrm{HCl}$ for $\mathrm{pH}$ adjustment) dosed for water purification (Zwiener et al., 2007). In addition to $\mathrm{Na}^{+}$, other cations (e.g., $\mathrm{Ca}^{2+}, \mathrm{K}^{+}$, $\mathrm{Mg}^{2+}$ ) were commonly detected in SPW matrix with a concentration level of milligram per liter. Remarkably, among these cations, $\mathrm{Ca}^{2+}$ turned out to be a second most abundant ion with a concentration of $\sim 0.5 \mathrm{mM}$, which mainly comes from the filling tap water $\left(\mathrm{Ca}^{2+}\right.$ in tap water is similar to that in SPWs according to our preliminary experiment).

Table 5.4 Characteristics of swimming pool water

\begin{tabular}{|c|c|c|c|c|c|}
\hline & Unit & $\begin{array}{l}\text { Analytical } \\
\text { instrument }\end{array}$ & Real SPW & $\begin{array}{l}\text { Synthetic } \\
\text { SPW }^{\text {b }}\end{array}$ & $\begin{array}{l}\text { Detection } \\
\text { limit }\end{array}$ \\
\hline $\mathrm{Na}$ & \multirow{4}{*}{$\mathrm{mg} / \mathrm{L}$} & \multirow{4}{*}{ ICP-OES } & $1062 \pm 90$ & 1175 & 0.1 \\
\hline $\mathrm{Ca}$ & & & $19.2 \pm 5.0$ & $0(40)$ & 0.1 \\
\hline $\mathrm{K}$ & & & $15.7 \pm 0.3$ & 20 & 0.1 \\
\hline $\mathrm{Mg}$ & & & $0.5 \pm 0.2$ & & 0.1 \\
\hline $\mathrm{Fe}$ & \multirow{8}{*}{$\mu \mathrm{g} / \mathrm{L}$} & \multirow{8}{*}{ ICP-MS } & $68.4 \pm 6.0$ & & 0.5 \\
\hline $\mathrm{Mn}$ & & & $0.2 \pm 0.3$ & & 0.5 \\
\hline $\mathrm{Cu}$ & & & $3.4 \pm 3.9$ & & 0.5 \\
\hline $\mathrm{Sr}$ & & & $37.1 \pm 5.7$ & & 0.5 \\
\hline $\mathrm{Zn}$ & & & $0.3 \pm 0.3$ & & 0.5 \\
\hline $\mathrm{Li}$ & & & $1.2 \pm 0.2$ & $100^{c}$ & 0.5 \\
\hline $\mathrm{Al}$ & & & $38.0 \pm 24.5$ & & 0.5 \\
\hline $\mathrm{B}$ & & & $65.6 \pm 22.2$ & 100 & 0.5 \\
\hline $\mathrm{F}$ & \multirow{7}{*}{$\mathrm{mg} / \mathrm{L}$} & \multirow{7}{*}{ IC } & $<0.2$ & & 0.2 \\
\hline $\mathrm{Cl}$ & & & $1953 \pm 71$ & $\begin{array}{c}1775 \\
(1864)\end{array}$ & 1 \\
\hline $\mathrm{NO}_{2}$ & & & $<1$ & & 1 \\
\hline $\mathrm{NO}_{3}$ & & & $20.8 \pm 1.0$ & 20 & 1 \\
\hline $\mathrm{SO}_{4}$ & & & $58.0 \pm 12.0$ & 60 & 1 \\
\hline $\mathrm{PO}_{4}$ & & & $<2$ & & 2 \\
\hline $\mathrm{Br}$ & & & $<1$ & $41^{\mathrm{c}}$ & 1 \\
\hline
\end{tabular}




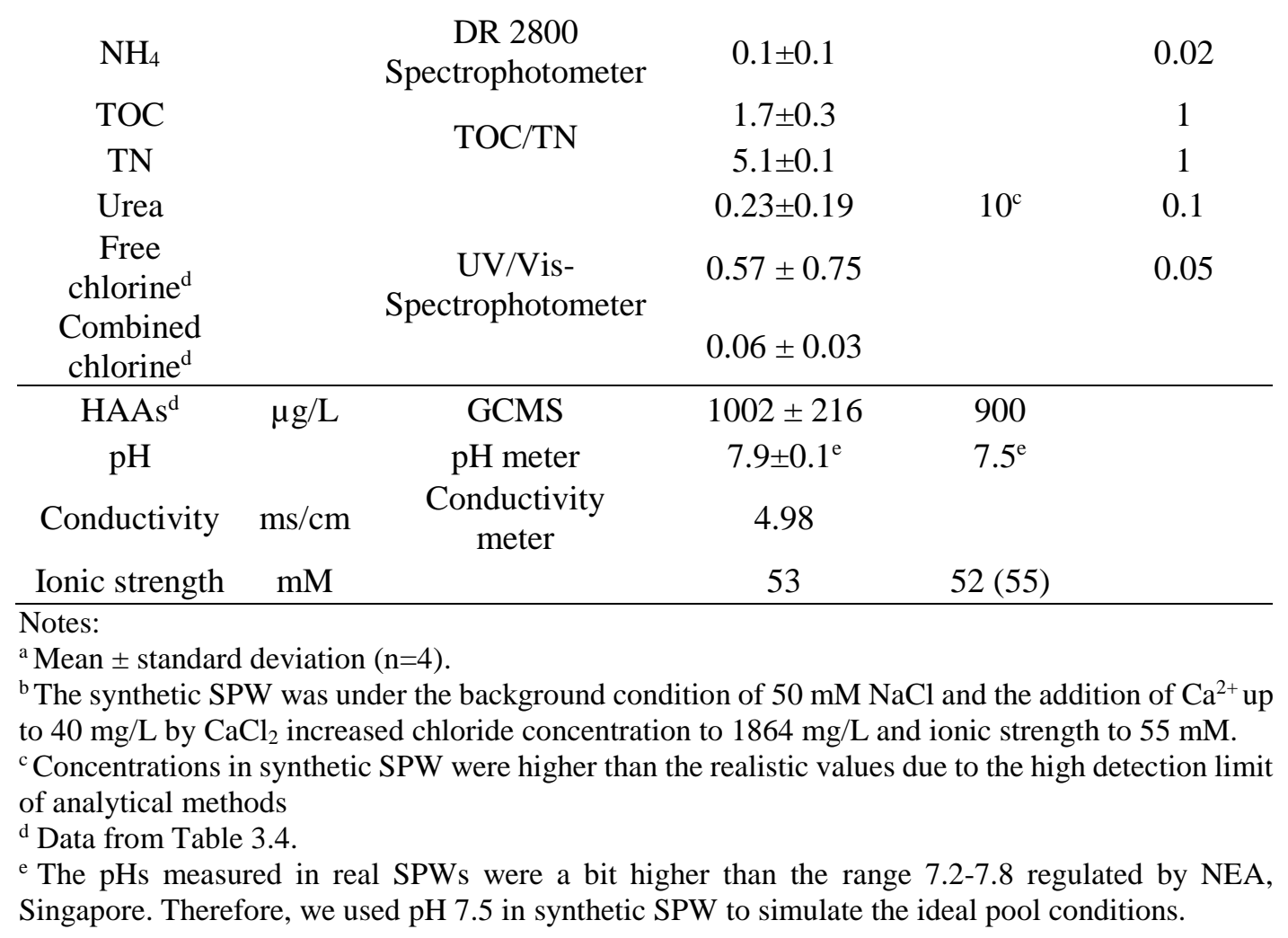

\subsubsection{Rejection of inorganic solutes by membranes}

\subsubsection{Real SPW}

The rejection of inorganic components in real SPWs was measured for both NF270 (Figure 5.2A) and NF90 (Figure 5.2B). The rejection data demonstrated two remarkable trends: 1$)$ neutral compounds, e.g. boron, had low rejections $(<30 \%)$ by both membranes; 2) the rejection of charged ionic species increased dramatically with the increased valence state for both cations and anions. For NF270 (Figure 5.2A), the maximum rejections of monovalent ions reached $\sim 50 \%$, while those for divalent ions were in the range of 55 to nearly $100 \%$. These results reveal the important role of electrostatic interaction for NF membranes, which is in good 
agreement with other literature studies (Bartels et al., 2005; Childress and Elimelech, 2000; Su et al., 2006).
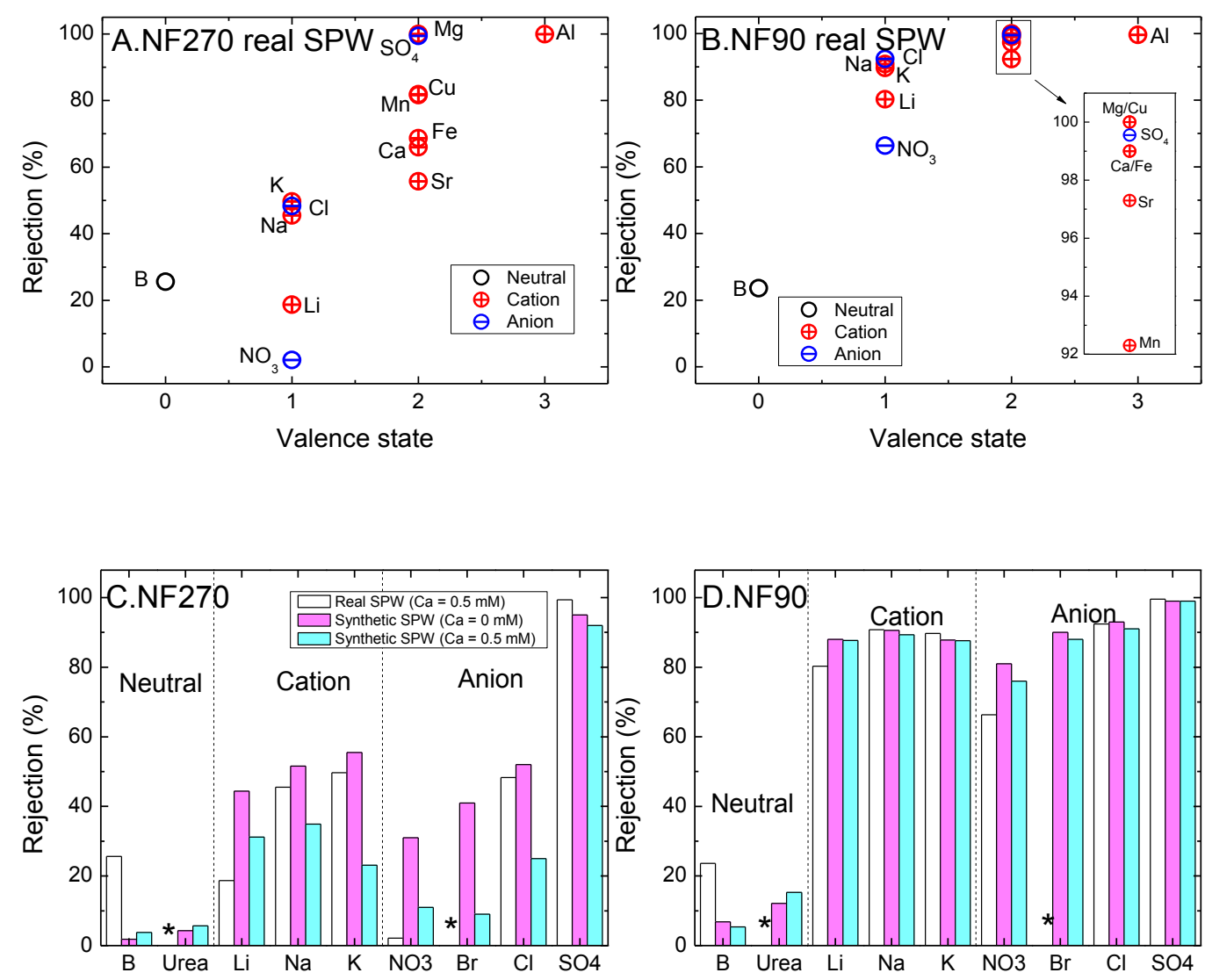

Figure 5.2 The rejection of components in real and synthetic SPWs by NF270 and NF90.

* The rejection data for urea in real SPW was not available and bromide ion in real SPW was not detectable $(<1 \mathrm{mg} / \mathrm{L})$.

NF90 demonstrated a similar rejection trend of higher rejection for ions with greater valence state (Figure 5.2B). Compared to NF270, NF90 had consistently higher rejection. In addition, the rejection data for NF90 were less scattered. NF90 had a smaller average pore radius of $0.31 \mathrm{~nm}$, which implies a more important role of size exclusion for this membrane compared to $\mathrm{NF} 270$ (pore radius of $0.40 \mathrm{~nm}$ ). The 
current study confirms the importance of both electrostatic interaction and size exclusion on membrane rejection.

\subsubsection{Comparing real and synthetic SPWs}

Figure 5.2C compares the rejection of different species in real and synthetic SPWs for NF270, where the synthetic SPW was dosed either 0 or $0.5 \mathrm{mM} \mathrm{Ca}^{2+}$. The significant variations in the rejection values observed under the three different solution conditions reveal the critical role of matrix effect for NF270. In particular, the addition of $0.5 \mathrm{mM} \mathrm{Ca}^{2+}$ into the synthetic SPW reduced the rejection of most ionic species significantly. In contrast, the matrix effect was much less obvious for the tighter membrane NF90 that experiences a stronger size exclusion effect (Figure 5.2D). The synthetic SPW filtration by NF90 and NF270 over a wider $\mathrm{Ca}^{2+}$ concentration range of 0-1 mM further supports these observations (Figure 5.3). The ubiquitous presence of $\mathrm{Ca}^{2+}$ in SPWs and its great influence on membrane rejection behaviour prompt us to conduct a systematic evaluation of the mechanistic role of $\mathrm{Ca}^{2+}$ in rejecting HAAs in SPWs (Section 5.3.3).
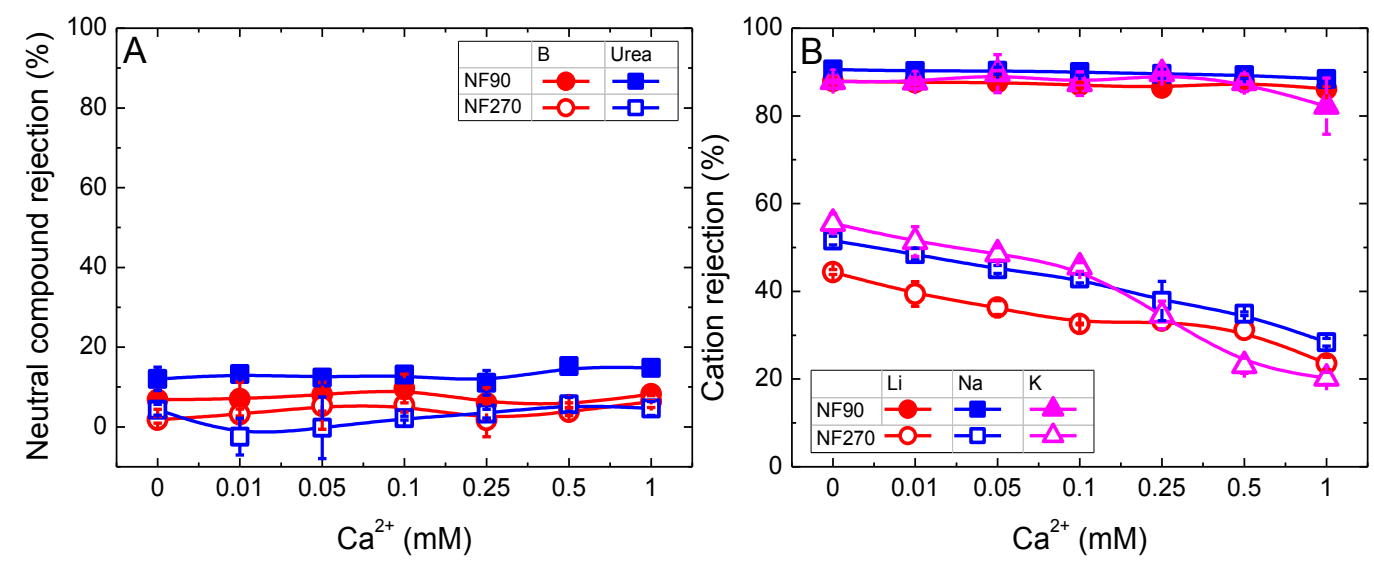


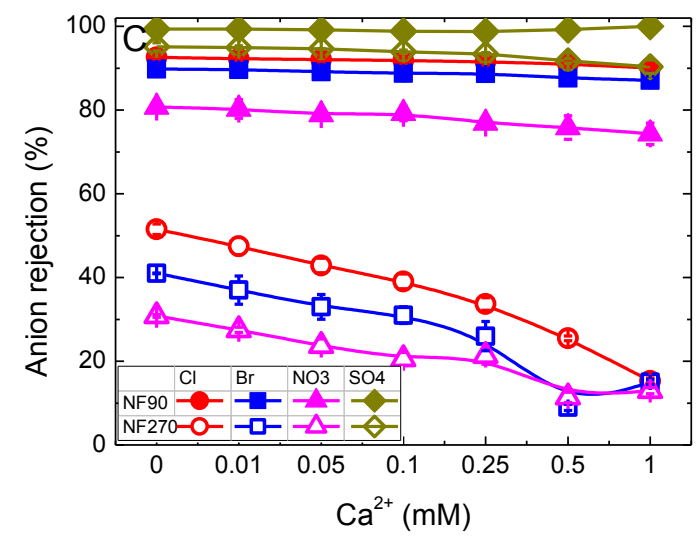

Figure 5.3 Effect of calcium ions on rejections of neutral compounds, cations, and anions by NF90 and NF270

\subsubsection{Rejection of HAAs by membranes}

\subsubsection{Effect of $\mathrm{Ca}^{2+}$ on HAA rejection}

Figure 5.4A,B show the effect of $\mathrm{Ca}^{2+}$ on HAA rejection by NF270 and NF90, respectively. To avoid the overcrowding of the figures, HAAs were grouped into MHAAs, D-HAAs, and T-HAAs based on the number of halogen atoms. HAA rejection by NF90 (> 95\%) was not significantly affected by the degree of halogenation or the addition of $\mathrm{Ca}^{2+}$. This observation can be attributed to the dominant role of size exclusion: the radii of HAAs range from $0.21-0.27 \mathrm{~nm}$ and are nearly comparable to the pore radius of NF90 $(0.31 \mathrm{~nm})$. On the contrary, the rejection by the looser NF270 membrane (pore radius of $0.40 \mathrm{~nm}$ ) followed the order of M-HAAs < D-HAAs < T-HAAs, which correlates well with the degree of halogenation (or equivalently the molecular size). Increasing $\mathrm{Ca}^{2+}$ concentration from 0 to $1 \mathrm{mM}$ caused the membrane rejection to decrease by approximately $20 \%$. 
The effect of $\mathrm{Ca}^{2+}$ on the rejection of HAAs is consistent with that on the rejection of inorganic species reported in Section 5.3.2.

The effect of $\mathrm{Ca}^{2+}$ on membrane rejection can be potentially attributed to its interaction with either HAAs or membrane surface. Both HAAs and the polyamide $\mathrm{NF}$ membranes contain carboxylic groups $\left(-\mathrm{COO}^{-}\right)$, which provide ligand sites for binding with $\mathrm{Ca}^{2+}$ (Herzberg et al., 2009; Jin et al., 2009; Mi and Elimelech, 2010; Motsa et al., 2014). Accordingly, three distinct mechanisms are plausible (Figure 5.4C): 1) the formation of HAA-Ca ${ }^{2+}$ complex, resulting in reduced charge density of HAAs together with a change in the size of the dissolved species (HAA-Ca ${ }^{2+}$ induced effect); 2) the formation of membrane-Ca ${ }^{2+}$ complex, which affects the pore size of the membrane (membrane- $\mathrm{Ca}^{2+}$ induced size exclusion effect); and 3) the formation of membrane- $\mathrm{Ca}^{2+}$ complex, which results in a partial neutralization of membrane surface charge (membrane-Ca ${ }^{2+}$ induced charge interaction effect). These mechanisms are further resolved through Section 5.3.3.2-5.3.3.4.
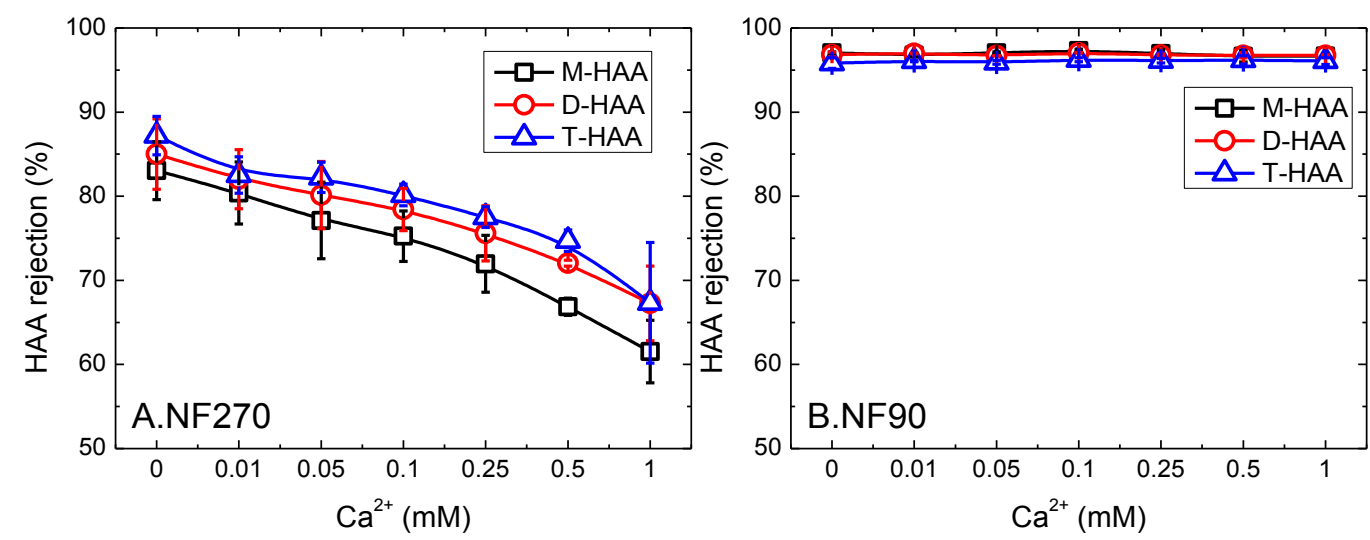
(1) $\mathrm{HAA}-\mathrm{Ca}^{2+}$ Complex

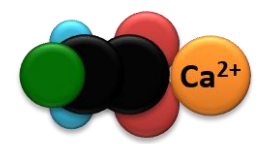

$\mathrm{Ca}^{2+}$

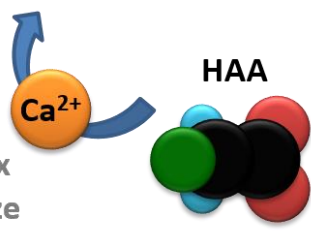

(3) Membrane-Ca ${ }^{2+}$ Complex $\rightarrow$

(2) Membrane-Ca ${ }^{2+}$ Complex

$\rightarrow$ altered pore size

charge neutralization

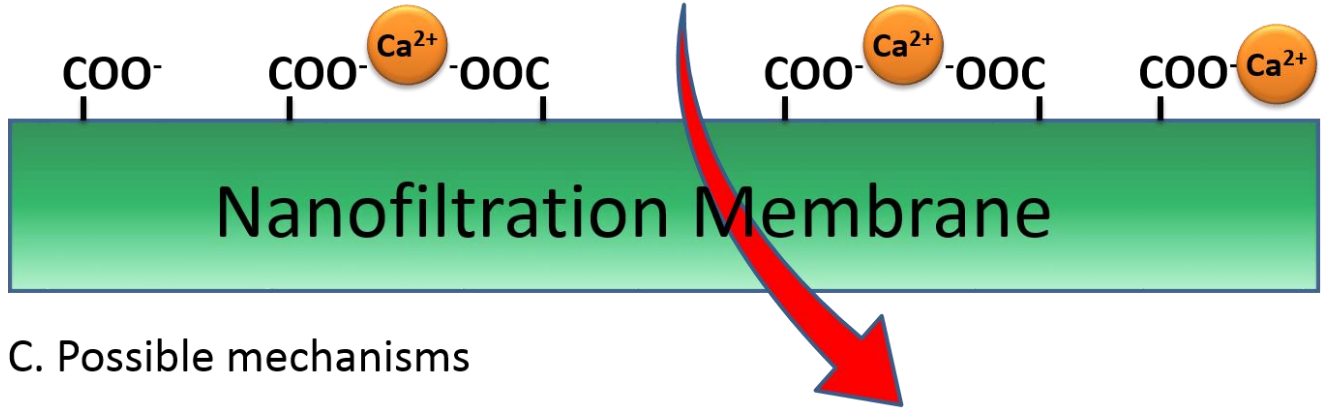

Figure 5.4 Effect of calcium ions on HAA rejection. Rejection data for NF270 (A) and NF90 (B) using synthetic SPW at pH 7.5. A conceptual diagram (C) illustrates the three possible mechanisms involved (1. HAA-Ca ${ }^{2+}$ induced effect; 2 . membrane$\mathrm{Ca}^{2+}$ induced size exclusion effect; and 3 membrane- $\mathrm{Ca}^{2+}$ induced charge interaction effect).

\subsubsection{HAA-Ca ${ }^{2+}$ induced effect}

Figure 5.5 shows the potential formation of $\mathrm{HAA}-\mathrm{Ca}^{2+}$ complex on the basis of the following chemical speciation analysis,

$$
\begin{gathered}
\mathrm{CR}_{3} \mathrm{COOH}=\mathrm{CR}_{3} \mathrm{COO}^{-}+\mathrm{H}^{+}, \mathrm{K}_{a}=\frac{\left[\mathrm{CR}_{3} \mathrm{COO}^{-}\right] \cdot\left[\mathrm{H}^{+}\right]}{\left[\mathrm{CR}_{3} \mathrm{COOH}\right]} \\
\mathrm{CR}_{3} \mathrm{COO}^{-}+\mathrm{Ca}^{2+}=\mathrm{CR}_{3} \mathrm{COOCa}^{+}, \mathrm{K}_{1}=\frac{\left[\mathrm{CR}_{3} \mathrm{COOCa}^{+}\right]}{\left[\mathrm{CR}_{3} \mathrm{COO}^{-}\right] \cdot\left[\mathrm{Ca}^{2+}\right]}
\end{gathered}
$$

where $\mathrm{CR}_{3} \mathrm{COO}^{-}$represents an $\mathrm{HAA}$ anion $(-\mathrm{R}=-\mathrm{H}$, $-\mathrm{Cl}$, or $-\mathrm{Br})$, and $\mathrm{K}_{\mathrm{a}}$ and $\mathrm{K}_{1}$ are the acidity constant and stability constant, respectively. The bridging of two HAA molecules by $\mathrm{Ca}^{2+}\left(\right.$ e.g., $\left.\left(\mathrm{CR}_{3} \mathrm{COO}\right)_{2} \mathrm{Ca}\right)$ is not considered due to the relatively low HAA concentrations $(\sim$ or $<1 \mu \mathrm{M}$, Table 5.1). As indicated in Figure 5.5, the two 
M-HAAs (chloroacetic acid and bromoacetic acid) exist predominantly in their anionic forms (only $<0.2 \%$ as $\mathrm{CH}_{2} \mathrm{ClCOOCa}^{+}$and $<0.4 \%$ as $\mathrm{CH}_{2} \mathrm{BrCOOCa}^{+}$for $\mathrm{Ca}^{2+}$ concentration up to $1 \mathrm{mM}$ ). Compared to acetic acid, HAAs have smaller $\mathrm{pK}_{\mathrm{a}}$ values (Table 5.1) as a result of the strong electron withdrawing effect of the halogen atoms, leading to a weaker binding with $\mathrm{H}^{+}$(Reusch, 2013; Stumm and Morgan, 2012). Similarly, HAAs had weaker binding with $\mathrm{Ca}^{2+}$ as indicated by their smaller $\log \mathrm{K}_{1}$ compared to that of acetic acid (data from NIST database). Despite that the stability constants for D-HAAs and T-HAAs are not available in the literature, their abilities to form HAA-Ca ${ }^{2+}$ complex are even weaker as more halogens further reduce the charge density of $-\mathrm{COO}^{-}$and therefore reduce the binding energy between $\mathrm{Ca}^{2+}$ and HAA anions. Therefore, the mechanism of HAA- $\mathrm{Ca}^{2+}$ complex formation is inadequate to explain the rejection behaviour presented in Figure 5.4.

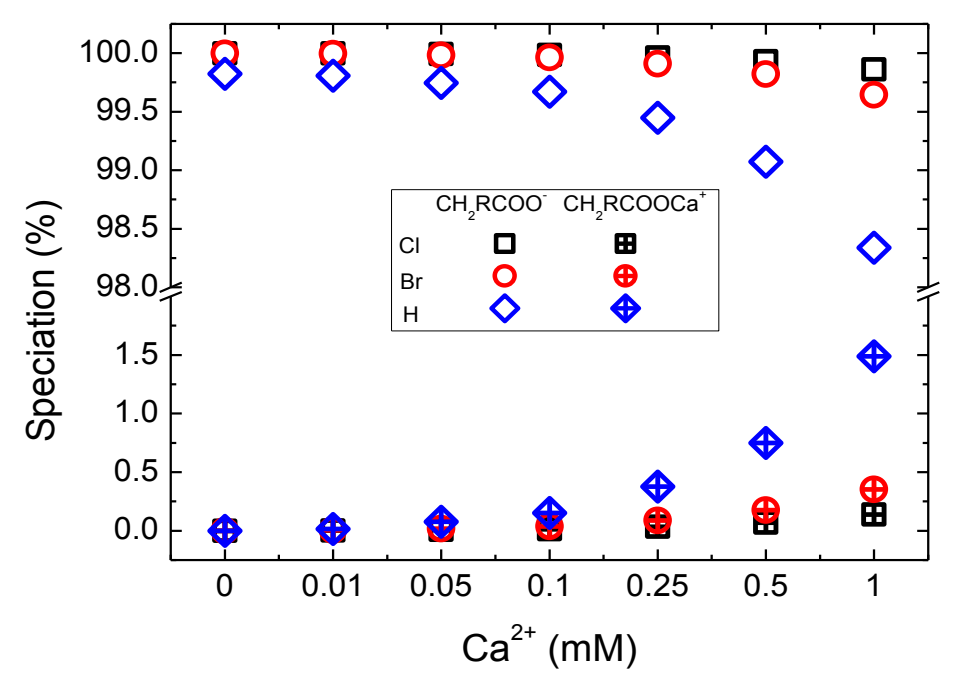

Figure 5.5 Species distribution of chloroacetic acid, bromoacetic acid, and acetic acid in the presence of calcium ions. The log values of stability constants for calcium-based complexes are $0.14,0.55$, and 1.18 , respectively. 


\subsubsection{Membrane-Ca ${ }^{2+}$ induced size exclusion effect}

Early studies have reported that the membrane pore size can be potentially affected by the solution chemistry (Childress and Elimelech, 2000). To determine whether $\mathrm{Ca}^{2+}$ can lead to a change in the membrane pore size, we performed rejection tests of seven surrogate molecules (glycerol, erythritol, xylose, glucose, maltose, sucrose, and raffinose) over a $\mathrm{Ca}^{2+}$ concentration range of $0-1 \mathrm{mM}$. The neutral hydrophilic nature of these surrogate compounds has allowed their use as molecular probes for determining membrane pore size (Nghiem et al., 2004) and for assessing the size exclusion effect (see details in last chapter). $\mathrm{Ca}^{2+}$ did not have a significant effect on the rejection of the surrogates for both NF270 (Figure 5.6A) and NF90 (Figure 5.6B). The water flux also maintained nearly constant over the entire $\mathrm{Ca}^{2+}$ concentration range (Figure 5.7). Accordingly, the average pore size of the membranes, calculated in accordance to Nghiem et al. (2004), was not affected by the presence of $\mathrm{Ca}^{2+}$ (Figure 5.6C). Therefore, the mechanism of membrane-Ca $\mathrm{Ca}^{2+}$ induced size exclusion effect is unable to explain the reduced HAA rejection at higher $\mathrm{Ca}^{2+}$ concentration (Figure 5.4A).
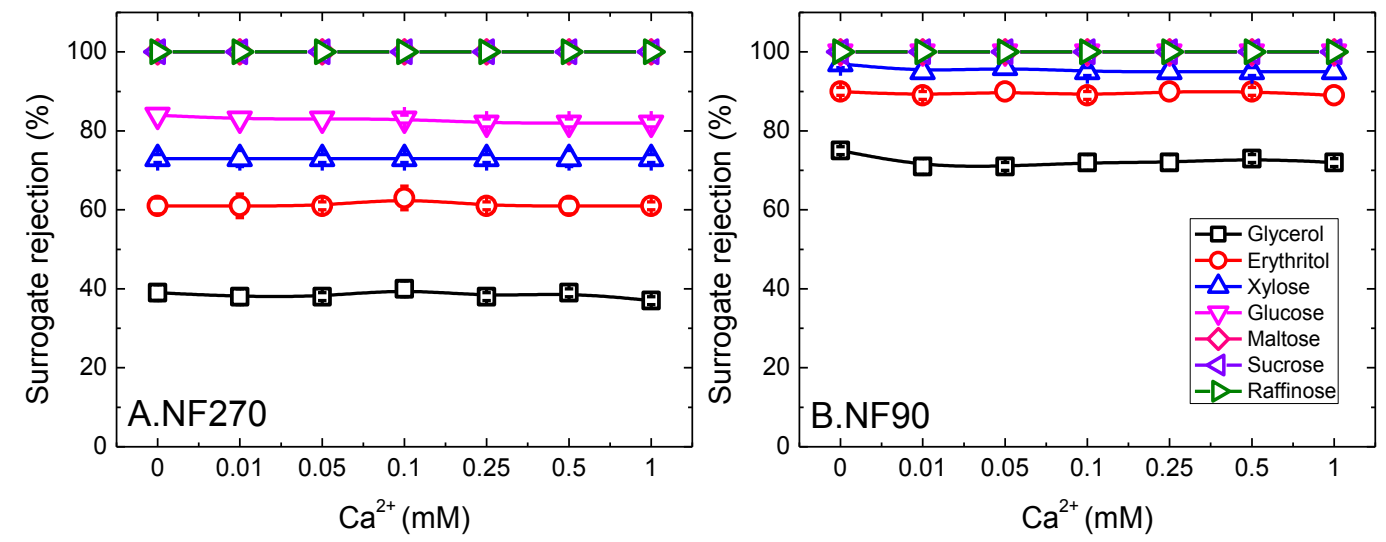


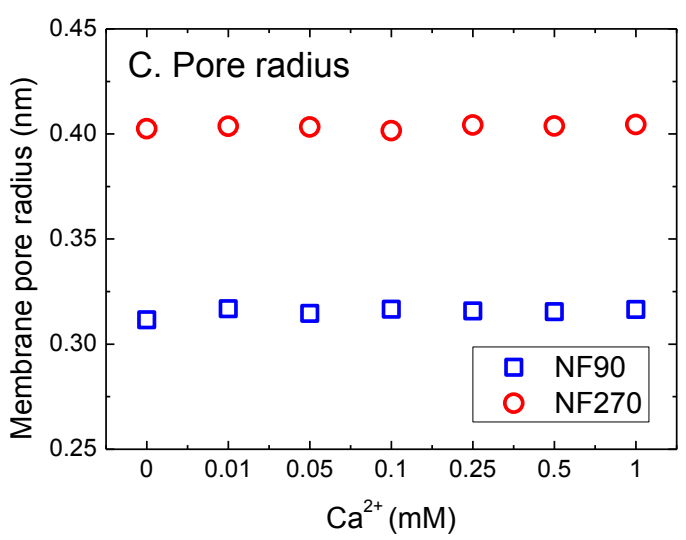

Figure 5.6 Effect of calcium ions on surrogate rejection by NF270 (A) and NF90 (B), and membrane pore size (C) calculated in accordance to Nghiem et al. (2004).

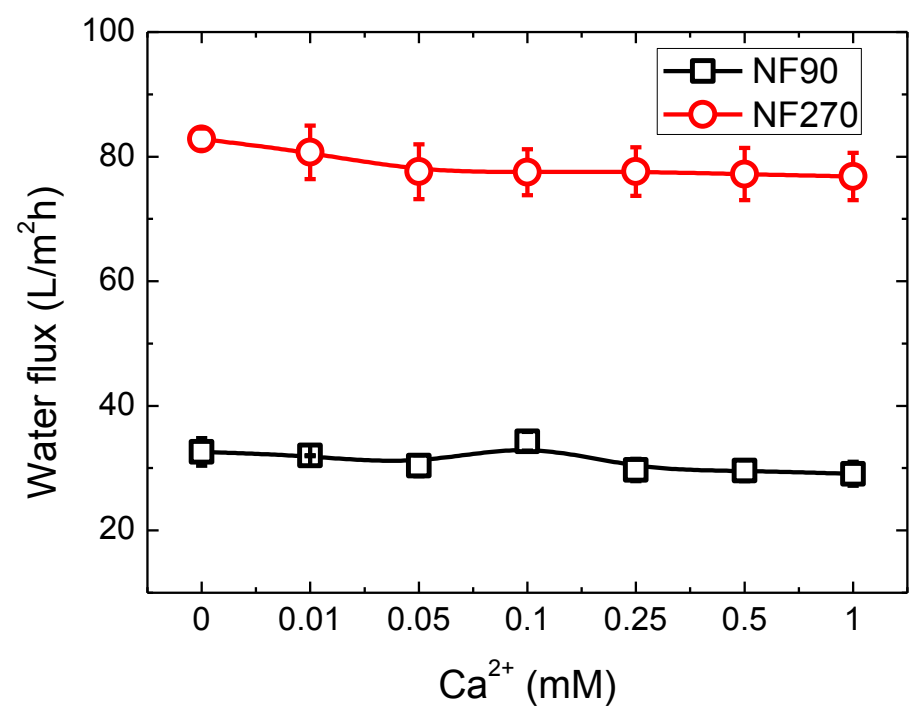

Figure 5.7 Effect of calcium ions on water flux (for surrogate filtration tests)

\subsubsection{Membrane-Ca ${ }^{2+}$ induced charge interaction effect}

Figure 5.8A demonstrates that both membrane surfaces became less negatively charged with the increasing $\mathrm{Ca}^{2+}$ concentrations, supporting our hypothesis that $\mathrm{Ca}^{2+}$ affects the membrane rejection by altering their surface charge properties (Figure 
5.4C). Yoon et al. (2005) and Childress and Elimelech (1996) also found that $\mathrm{Ca}^{2+}$ possesses a much stronger charge neutralization ability compared to other electrolytes, e.g., $\mathrm{KCl}, \mathrm{K}_{2} \mathrm{SO}_{4}$ and $\mathrm{Na}_{2} \mathrm{SO}_{4}$, attributed to its stronger binding to the membrane surface. The binding of $\mathrm{Ca}^{2+}$ to membrane surface is further supported by our XPS analysis, with much intense calcium signals detected for the membranes exposed to 0.1 and $1 \mathrm{mM} \mathrm{Ca}^{2+}$ compared to the control (no $\mathrm{Ca}^{2+}$ ) (Figure 5.8B-D). It can be explained by the interaction between $\mathrm{Ca}^{2+}$ and the ligand groups (particularly -COO-) (Herzberg et al., 2009; Jin et al., 2009; Mi and Elimelech, 2010; Motsa et al., 2014). Indeed, $\mathrm{Ca}^{2+}$ binding has been used to characterize the relative abundance of $-\mathrm{COO}^{-}$groups contained in polyamide membranes (Do et al., 2012c). In the current study, the effect of $\mathrm{Ca}^{2+}$ on zeta potential was more considerable for NF270 than NF90, which is attributed to the greater presence of -COO- groups in NF270.

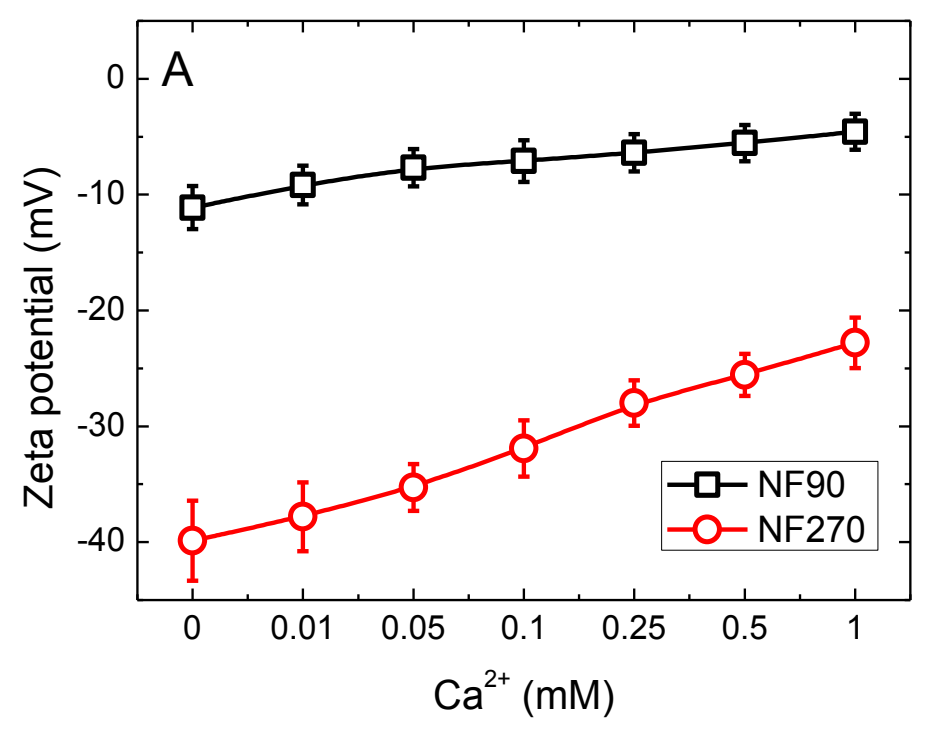



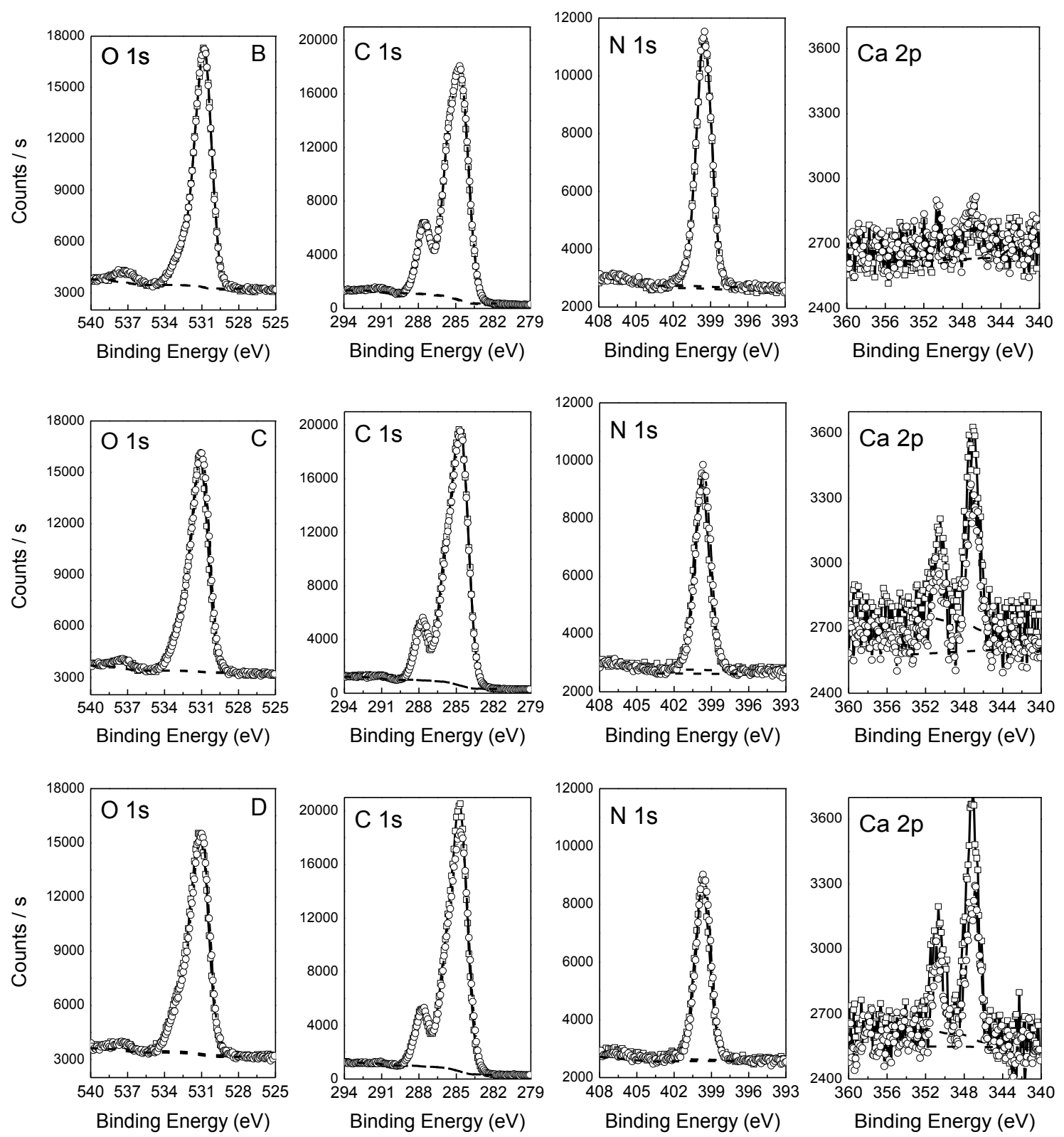

Figure 5.8 Effect of calcium ions on zeta potential for NF90 and NF270 (A). High resolution XPS spectra (duplicate analysis) for NF270 exposed in $\mathrm{Ca}^{2+}$ solutions at different concentrations of $0 \mathrm{mM}$ as control (B), $0.1 \mathrm{mM}$ (C), and $1 \mathrm{mM}$ (D). The minor Ca peak for the control membrane was caused by the $0.001 \mathrm{mM} \mathrm{CaCl}_{2}$ rinse solution. 
5.3.3.5 Combined effects of size exclusion and charge interaction on HAA rejection

To further assess the role of size exclusion and charge interaction, we measured HAA rejections by NF270 at $\mathrm{pH} 3.5$ with or without $\mathrm{Ca}^{2+}$. At $\mathrm{pH} 3.5$, the membrane is nearly neutral. In addition, the saturation of carboxylic groups with $\mathrm{H}^{+}$(i.e., $-\mathrm{COOH}$ instead of $-\mathrm{COO}^{-}$) prevents the binding of $\mathrm{Ca}^{2+}$ to the membrane. Therefore, this $\mathrm{pH}$ condition effectively suppresses the charge interaction and thus establishes a baseline case of the size exclusion effect. A plot of HAA rejection at $\mathrm{pH} 3.5$ as a function of the molecular radius (Figure 5.9) indicates an increased rejection that benefited from the enhanced size exclusion for the bulkier molecules (with molecular radius increased from $\sim 0.21$ to $\sim 0.27 \mathrm{~nm}$ ). The presence of $\mathrm{Ca}^{2+}$ did not significantly affect the HAA rejection at this low $\mathrm{pH}$ (see Figure 5.10) since $\mathrm{Ca}^{2+}$ is outcompeted by $\mathrm{H}^{+}$for binding to the membrane ligand sites.

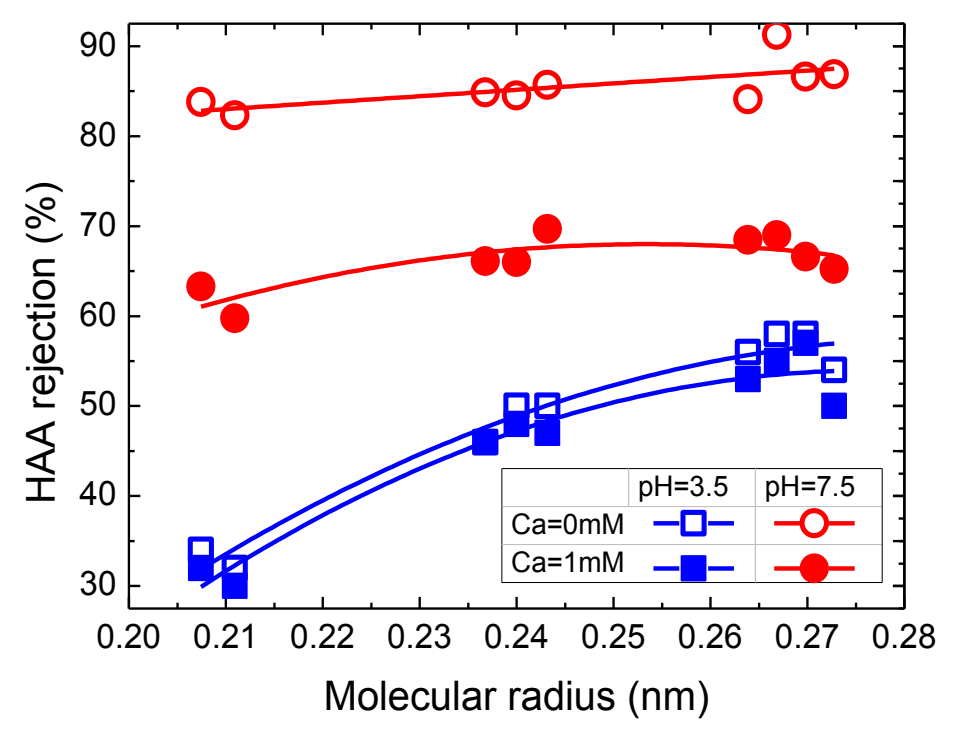

Figure 5.9 HAA rejection as a function of molecular radius at $\mathrm{pH} 3.5$ and 7.5 in the presence of calcium ions by NF270. 


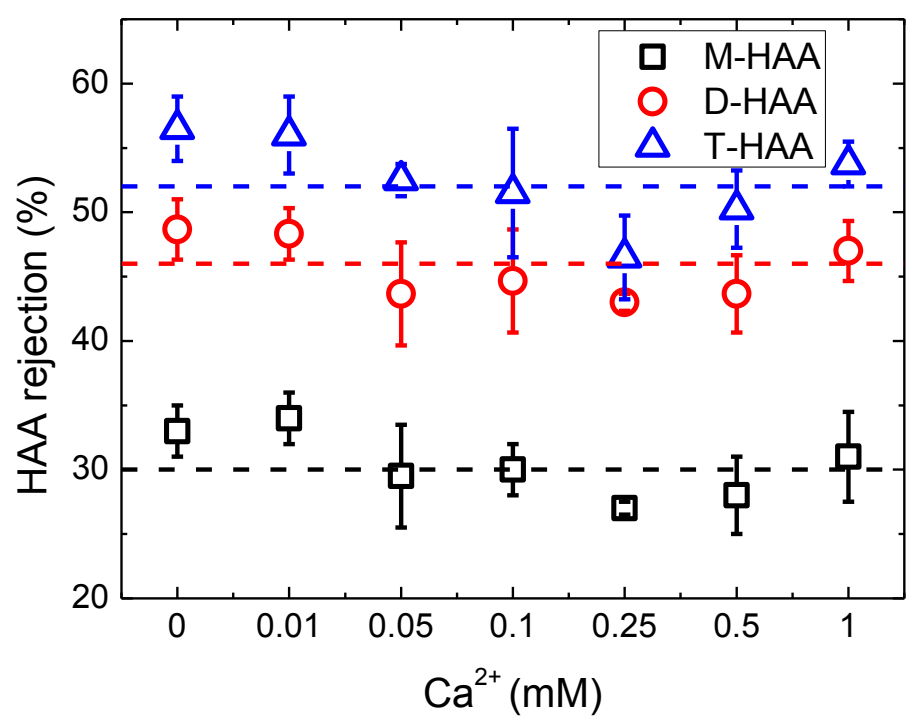

Figure 5.10 Effect of calcium ions on HAA rejection at $\mathrm{pH} 3.5$ by NF270. The dash lines represent the average rejection under various concentrations of calcium ions.

In the absence of $\mathrm{Ca}^{2+}$, increasing $\mathrm{pH}$ from 3.5 to 7.5 dramatically enhanced the HAA rejection from $30-55 \%$ to $>80 \%$. Compared to the near-neutral membrane surface at pH 3.5 (zeta potential $\sim-5 \mathrm{mV}$ ), NF270 became highly negatively charged at $\mathrm{pH} 7.5$ (zeta potential -36 mV) (Figure 4.4). Thus, the difference in rejection between $\mathrm{pH}$ 3.5 and $\mathrm{pH} 7.5$ characterizes the effect of charge interaction on HAA removal. This difference became larger at a lower molecular radius, which indicates that charge interaction has to assume a more predominant role for the smaller molecules (in the presence of weaker size exclusion). Similarly, HAA rejection has a stronger dependence on the size exclusion effect when there was a weaker charge interaction, as reflected by the steeper slope of the rejection curve at $\mathrm{pH} 3.5$ compared to that at pH 7.5. 
The HAA rejection at $\mathrm{pH} 7.5$ with $1 \mathrm{mM} \mathrm{Ca}^{2+}$ was significantly lower than that at the same $\mathrm{pH}$ without $\mathrm{Ca}^{2+}$. Nevertheless, it was still substantially higher than that at pH 3.5 (or $\left[\mathrm{H}^{+}\right]=0.32 \mathrm{mM}$ ). The current study shows that both $\mathrm{Ca}^{2+}$ and $\mathrm{H}^{+}$ decreased HAA rejection by binding to the carboxylic groups on the membrane, though $\mathrm{H}^{+}$had a more severe effect by completely neutralizing the membrane surface charge at $\mathrm{pH} 3.5$.

\subsubsection{Implications}

As discussed in Section 5.3.3.1-5.3.3.4, the membrane-Ca ${ }^{2+}$ induced charge interaction effect is the main mechanism that contributes to the reduced rejection of HAAs and inorganic ions (e.g., $\mathrm{Na}^{+}$and $\mathrm{Cl}^{-}$) in the presence of $\mathrm{Ca}^{2+}$. To further consolidate this conclusion, we simulated the conductivity rejection (same as $\mathrm{Cl}^{-}$and $\mathrm{Na}^{+}$) by a transport model developed by Bowen et al. (1997), only considering the changed membrane surface charge in the presence of $\mathrm{Ca}^{2+}$ (see Appendix A2). This model quantitatively links the ion rejection with the zeta potential of the membranes. In the feed water containing $100 \mu \mathrm{g} / \mathrm{L}$ of each HAA, $50 \mathrm{mM} \mathrm{NaCl}$, and $0 \sim 1 \mathrm{mM}$ $\mathrm{CaCl}_{2}$, we assumed that the distribution of $\mathrm{Na}^{+}$and $\mathrm{Cl}^{-}$was not affected by HAAs and $\mathrm{CaCl}_{2}$ due to their relatively low concentrations, and that $\mathrm{CaCl}_{2}$ only affected the membrane surface charge. The condition was therefore simplified as the single salt (i.e., $\mathrm{NaCl}$ ) passed through a membrane with changing membrane surface charge. The agreement between measured and simulated conductivity rejection confirms the reduced rejection is due to the neutralization effect of $\mathrm{Ca}^{2+}$ on membrane surface charge (see Figure 5.11). 


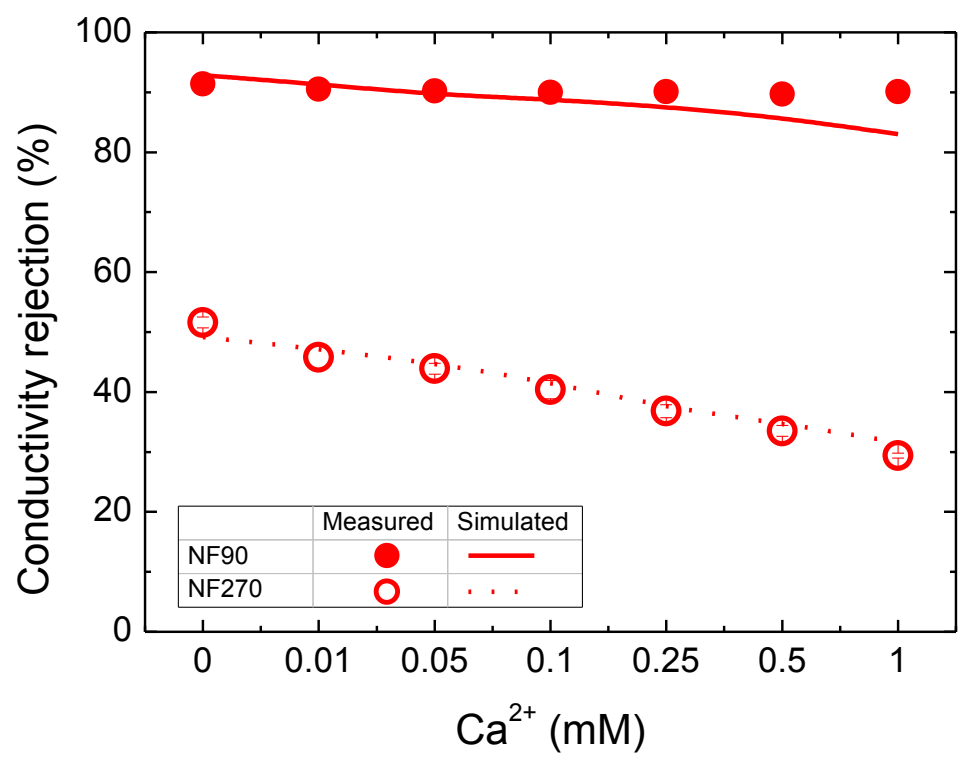

Figure 5.11 Comparison of measured and simulated conductivity rejection in the presence of calcium ions

\subsection{Conclusions}

Membrane filtration is an effective technology to remove HAAs. For the tight NF90 membrane whose pore size is comparable to HAAs, their rejection was consistently high (> 95\%) and was not significantly affected by the water matrix, reflecting the dominance of size exclusion effect. However, the HAA rejection by loose NF270 membrane at $\mathrm{pH} 7.5$ was significantly reduced at the increased concentration of calcium, a commonly detected ion in SPWs. This matrix effect can be attributed to the formation of membrane- $\mathrm{Ca}^{2+}$ complex (as shown by the XPS results) and the resulting partial charge neutralization of the membrane surface (supported by the zeta potential measurements). In contrast, the rejection of neutral hydrophilic molecular probes was not affected by the presence of $\mathrm{Ca}^{2+}$, suggesting that the 
formation of membrane- $\mathrm{Ca}^{2+}$ complex did not affect the pore structure of the membrane. Thus, it was the weakened charge interaction, instead of size exclusion, that was responsible for the reduced rejection of HAAs in the case of NF270. The fundamental understanding of this matrix effect on contaminant rejection promotes the effective application of membrane technology to practical SPW treatment. In addition to SPWs, other water types, e.g., DW, wastewater, etc., should be applicable to this mechanistic interpretation as well. Nevertheless, the effectiveness of real application in any water types should be on the base of the comprehensive cognition of water matrix. 


\section{Chapter 6 Conclusions and Recommendations}

\subsection{Conclusions and contributions}

This study explored novel approaches for the control and treatment of DBPs in SPWs. It investigated the appropriateness of applying alternative disinfectants to minimize DBP formation from the source and using membrane technology to remove already generated HAAs from the simulated SPWs. The effect of calcium ions (a ubiquitous species in SPWs) on HAA rejection by membranes has been systematically explored. The fundamental understanding of this matrix effect on contaminant rejection promotes the effective application of membrane technology to practical SPW treatment. The important findings in this research can be concluded as follows.

1) This study reviewed the latest research progress on the occurrence, formation, and treatment of DBPs in the context of SPWs. More specifically, it provided comprehensive knowledge and information from multiple aspects, e.g., DBP regulations in different regions, formation mechanisms related with different disinfectants, precursors and other factors, toxicity and carcinogenicity, human exposure assessment reflected by biomarkers or epidemiological evidence, and latest approaches for the control and treatment of DBPs. This critical review provided the potential directions of minimizing the DBP formation and handling the formed DBPs for DBP control. It demonstrates that chlorine/bromine stabilized disinfectants carry the potential to sustain the sufficient effective

disinfection species in pools, especially for those exposed under sunlight. 
However, the DBP formation from these alternative disinfectants is still unclear. In addition, membrane filtration turned out to be a promising approach to control DBPs and DBP precursors. The underlying mechanisms have not been comprehensively understood and most laboratory-scale investigations ignored many significant realistic conditions. These unknown and unclear situations trigger our curiosities and passions for further investigations.

2) $\mathrm{NaClO}$ is the most commonly used disinfectant in pool treatment system. Pools in tropical regions usually suffer from the strong sunlight irradiation which degrades the free chlorine rapidly. In addition, more pools start to adopt the recirculation of SPWs, which intensifies the DBP accumulation issue. Given these potential drawbacks of using $\mathrm{NaClO}$ in the tropical environment, two alternative organic-based disinfectants, TCCA and $\mathrm{BCDMH}$, were investigated and compared to $\mathrm{NaClO}$ in terms of its self-degradation and the formation of DBPs, including THMs and HAAs, under simulated tropical climate conditions. The result revealed that halogen stabilizer, TCCA, has the advantages of slower free chlorine degradation and lower $\mathrm{DBP}$ concentration compared to $\mathrm{NaClO}$, and thus makes it a good alternative disinfectant. BCDMH was not recommended mainly due to the highly reactive disinfecting ingredient, $\mathrm{HBrO}$, which fails to sustain the continuous disinfection requirement. Total disinfectant dosage was the main factor that affects residual chlorine and THM/HAA formation regardless of different disinfectant dosing methods, e.g. shock dosing (one-time spiking) in the beginning and continuous dosing during the whole period. Two- 
stage second-order-kinetic-based models demonstrate a good correlation between the measured and predicted data for chlorine decay $\left(R^{2} \geqslant 0.95\right)$, THM $\left(R^{2} \geqslant 0.99\right)$ and HAA $\left(R^{2} \geqslant 0.83\right)$ formation. Higher temperature was found to enhance the DBP formation due to the temperature dependence of reaction rates. Thus, temperature control of pools, especially for those preferring higher temperatures (e.g. hydrotherapy and spa), should take both bather comfort and DBP formation potential into consideration. It is also observed that chlorine competition existed between different precursors from NOM in filling water and body fluids from pool users. Among the composition of human body fluids, uric acid, citric acid and hippuric acid were found as main precursors of HAA formation.

3) Recent studies report high concentrations of HAAs, a prevalent class of toxic DBPs, SPW. We investigated the removal of 9 HAAs by four commercial RO and NF membranes. Under typical SPW conditions (pH 7.5 and $50 \mathrm{mM}$ ionic strength), HAA rejections were $>60 \%$ for loose NF270 membrane and $\sim$ or $>$ $90 \%$ for tighter membranes (XLE, NF90 and SB50) as a result of the combined effects of size exclusion and charge repulsion. We further included 7 neutral hydrophilic surrogates as molecular probes to resolve the rejection mechanisms. In the absence of strong electrostatic interaction (e.g., $\mathrm{pH} 3.5$ ), the rejection data of HAAs and surrogates by various membranes fall onto an identical sizeexclusion curve when plotted against the hindrance parameter, i.e., the ratio of molecular radius over membrane pore radius. The independence of this SE curve 
on molecular structures and membrane properties reveals that the hindrance parameter is a more fundamental size-exclusion descriptor compared to molecular weight. An effective molecular size can be further used to take account for charge repulsion in addition to size exclusion. The current study provides valuable insights on the rejection of trace contaminants by $\mathrm{RO} / \mathrm{NF}$ membranes.

4) We investigated the removal of HAAs from SPWs by two nanofiltration membranes NF270 and NF90. The strong matrix effect (particularly by $\mathrm{Ca}^{2+}$ ) on membrane rejection prompts us to systematically investigate the mechanistic role of $\mathrm{Ca}^{2+}$ in HAA rejection. At typical SPW $\mathrm{pH}$ of 7.5 , NF90 maintained consistently high rejection of HAAs (> 95\%) with little influence by $\mathrm{Ca}^{2+}$, thanks to the dominance of size exclusion effect for this tight membrane (pore radius $0.31 \mathrm{~nm})$. In contrast, the rejections of both inorganic ions (e.g., $\mathrm{Na}^{+}$and $\mathrm{Cl}^{-}$) and HAA anions were decreased at higher $\mathrm{Ca}^{2+}$ concentration for NF270 (pore radius $\sim 0.40 \mathrm{~nm}$ ). Further tests show that the rejection of neutral hydrophilic molecular probes and the membrane pore size were not affected by $\mathrm{Ca}^{2+}$. Although $\mathrm{Ca}^{2+}$ is unable to form a strong complex with HAAs, we observed the binding of $\mathrm{Ca}^{2+}$ to NF270 together with a reduction in its surface charge. Therefore, the formation of membrane- $\mathrm{Ca}^{2+}$ complex, which weakens charge interaction effect, was responsible for the reduced HAA rejection. The current study reveals important mechanistic insights of the matrix effect on trace contaminant rejection, which is critical for a better understanding of their fate and removal in membrane-based treatment. 


\subsection{Recommendations and future work}

There are still several aspects that need further exploration to promote the full-scale application of these promising technologies. We have proposed the future work from the following aspects.

1) We have included several ionic species (e.g., $\mathrm{Na}^{+}, \mathrm{Ca}^{2+}$, etc.) and a neutral compound (i.e., urea) in the simulated SPW. However, apart from these, many other compounds, organic matters in particular, do exist in real SPWs, which have the potential to affect the HAA rejection by interacting with calcium ions or membrane surfaces. For example, organic compounds with carboxyl functional group (e.g., citric acid) may bind with some calcium ions, therefore weaken the binding between calcium and membrane surface. In addition, chlorine is a ubiquitous species in SPWs as well, therefore chlorine exposure of applied membranes should be considered. Cellulose acetate membrane (e.g., SB50) is chlorine tolerant while polyamide membranes (e.g., XLE, NF90, NF270) are chlorine intolerant. Hence, surface modification should be considered if polyamide membranes are applied. Another approach is to add a prior chlorine reduction process before the polyamide membrane setup. In this case, periodical membrane treatment may be preferred to prevent the waste of chemicals (e.g., chlorine) and to save the overall cost, as long as the water quality meets requirements. Membrane fouling should also be considered due to the existence of some suspended particles and solids in real SPWs. Therefore, the overall 
membrane performance for the DBP treatment should be a result of these combined effects. After clarifying all these potentially affecting factors, DBP rejection in real SPWs could be fully understood.

2) The weakness of membrane filtration compared to other treatment methods is the former actually only plays a role on contaminant enrichment. We should also pay special attention to the further treatment of DBP-contained retentate generated from the filtration process to make the effluent absolutely environmental-friendly. AOPs (e.g., UV-, $\mathrm{H}_{2} \mathrm{O}_{2}$ - or $\mathrm{O}_{3}$-based) could be used as a subsequent treatment process after the membrane filtration. A systematic treatment process for SPWs has been proposed in Figure 6.1. It contains two parts, one is similar to the conventional process mainly aiming at removing the conventional pollutants, the other as an advanced treatment is the combined process of membrane filtration and AOPs in order to remove robust micropollutants. We may suggest performing the latter process periodically since the formation of some DBPs takes certain time. The effluent from the AOP reactor should not contain these DBPs or contain DBPs with acceptable concentrations, thereafter returns back to the swimming pools. Otherwise, it is discharged to the sewer for further treatment. A pilot-scale setup with long-term testing could be attempted before full-scale applications. 


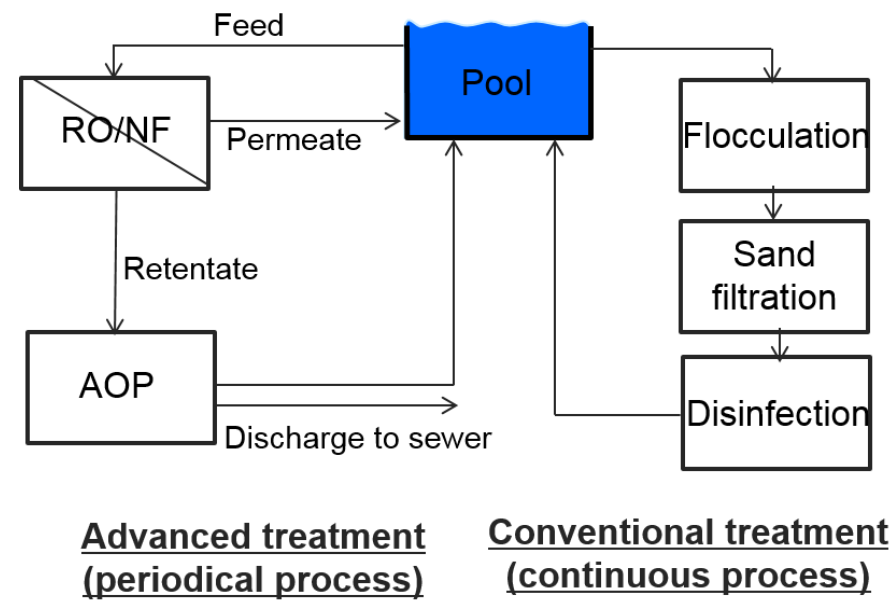

Figure 6.1 Proposed treatment process for SPWs 


\section{Appendix}

\section{A1. Transport model for uncharged solutes}

In the current study, the membrane pore radius was determined using the solute transport model for uncharged solutes, originally developed by Nghiem et al. (2004). In this model, the membrane rejection layer is assumed to have cylindrical pores with identical radius, and both the diffusive and convective contributions are accounted for:

$$
J_{s}=-K_{d} D_{\infty} \frac{d c}{d x}+K_{c} c V
$$

where $J_{s}$ is the solute flux $\left(\mathrm{mol} \cdot \mathrm{m}^{-2} / \mathrm{s}\right) ; D_{\infty}$ is the Stokes-Einstein bulk diffusion coefficient $\left(\mathrm{m}^{2} / \mathrm{s}\right) ; K_{d}$ and $K_{c}$ are the dimensionless hindered diffusive and convective coefficients, respectively (see Table A1 for the determination of $K_{d}$ and $\left.K_{c}\right) ; \boldsymbol{C}$ is the solute concentration in the membrane pores $\left(\mathrm{mol} / \mathrm{m}^{3}\right) ; x$ is the axial position along the membrane cylindrical pore $(\mathrm{m}) ; V$ is the effective average fluid velocity in membrane pores $(\mathrm{m} / \mathrm{s})$, i.e., the ratio of water flux $J_{v}$ over membrane porosity $\mathcal{E}$.

With the introduction of a dimensionless partitioning coefficient $\Phi$, Nghiem et al. (2004) obtained a useful expression of solute flux by integrating Eq. (1):

$$
J_{s}=\frac{\Phi K_{c} V C_{m}\left[1-\frac{C_{p}}{C_{m}} \exp (-P e)\right]}{1-\exp (-P e)}
$$


where $C_{m}$ and $C_{p}$ are solute concentrations just outside the membrane pores at the positions of $x=0$ and $x=L\left(\mathrm{~mol} / \mathrm{m}^{3}\right)$, respectively; $L$ is the membrane thickness (m). In Eq. (2), the dimensionless Peclet number $P \boldsymbol{e}$ and partitioning coefficient $\Phi$ are given by:

$$
\begin{gathered}
P e=\frac{K_{c} J_{v} L}{K_{d} \varepsilon D_{\infty}} \\
\Phi=\frac{c_{o}}{C_{m}}=\frac{c_{L}}{C_{p}}=(1-\lambda)^{2}
\end{gathered}
$$

where $c_{o}$ and $c_{L}$ are the solute concentrations just inside the membrane pores at the positions of $x=0$ and $x=L\left(\mathrm{~mol} / \mathrm{m}^{3}\right)$, respectively; $\lambda$ is the ratio of the solute radius $\left(r_{s}\right)$ to membrane pore radius $\left(r_{p}\right)$.

Since the solute flux is related to the solute permeate concentration by $J_{s}=V C_{p}$, Eq. (2) can be arranged to yield the following expression of the real rejection of the solute $R_{r}:$

$$
R_{r}=1-\frac{C_{p}}{C_{m}}=1-\frac{\Phi K_{c}}{1-\exp (-P e)\left(1-\Phi K_{c}\right)}
$$

where the real rejection $R_{r}$ is related to the observed rejection $R_{o}\left(=1-\frac{C_{p}}{C_{b}}\right)$ through film theory for concentration polarization by (Sutzkover et al., 2000):

$$
\ln \frac{1-R_{r}}{R_{r}}=\ln \frac{1-R_{o}}{R_{o}}-\frac{J_{v}}{k_{f}}
$$


The mass transfer coefficient $k_{f}$ was determined based on the water flux decline induced by the addition of salt solution compared to the salt-free feed (Nghiem et al., 2004; Sutzkover et al., 2000).

Table A1 Determination of hindered diffusive and convective coefficients

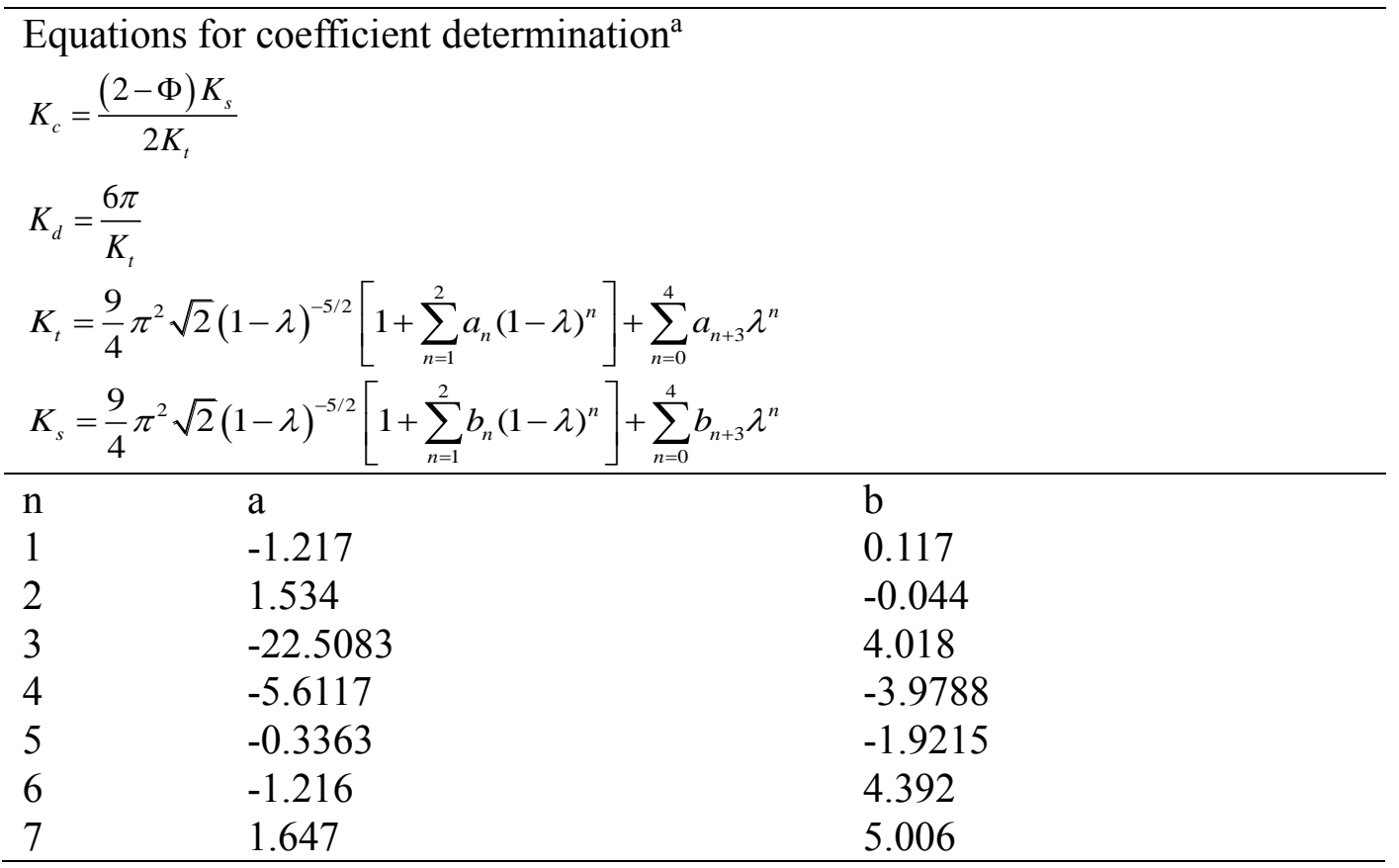

${ }^{\mathrm{a}}$ These equations cover the entire $\lambda$ range $(0 \leq \lambda \leq 1)$ (Bandini and Bruni, 2010; Nghiem et al., 2004). The symbols, including $K_{t}, K_{s}, a_{n}$, and $b_{n}$, are the coefficients of hindered factors (dimensionless).

List of symbols

$a_{n}, b_{n} \quad$ Coefficients, $\mathrm{n}=1,2, \ldots, 6$ (dimensionless)

c Solute concentration in the membrane pores $\left(\mathrm{mol} / \mathrm{m}^{3}\right)$

$c_{o} \quad$ Solute concentration inside the membrane pores at the positions of $x=0\left(\mathrm{~mol} / \mathrm{m}^{3}\right)$ 
$c_{L} \quad$ Solute concentration inside the membrane pores at the positions of $x=L\left(\mathrm{~mol} / \mathrm{m}^{3}\right)$

$C_{b} \quad$ Solute concentration in the bulk feed solution $\left(\mathrm{mol} / \mathrm{m}^{3}\right)$

$C_{m} \quad$ Solute concentration outside the membrane pores at the positions of $x=0\left(\mathrm{~mol} / \mathrm{m}^{3}\right)$

$C_{p} \quad$ Solute concentration outside the membrane pores at the positions of $x=L\left(\mathrm{~mol} / \mathrm{m}^{3}\right)$

$D_{\infty} \quad$ Stokes-Einstein bulk diffusion coefficient $\left(\mathrm{m}^{2} / \mathrm{s}\right)$

$J_{s} \quad$ Solute flux $\left(\mathrm{mol} \cdot \mathrm{m}^{-2} / \mathrm{s}\right)$

$J_{v} \quad$ Permeate water flux $(\mathrm{m} / \mathrm{s})$

$k_{f} \quad$ Mass transfer coefficient $(\mathrm{m} / \mathrm{s})$

$K_{c} \quad$ Hindered convective coefficient (dimensionless)

$K_{d} \quad$ Hindered diffusive coefficient (dimensionless)

$K_{t}, \mathrm{~K}_{s} \quad$ Coefficients of Hindered factors (dimensionless)

$L \quad$ Membrane thickness (m)

$\mathrm{Pe} \quad$ Membrane Peclet number (dimensionless)

$r_{p} \quad$ Membrane pore radius (m)

$r_{s} \quad$ Solute radius $(\mathrm{m})$

$R_{r} \quad$ Real rejection of the solute (dimensionless)

$R_{o} \quad$ Observed rejection of the solute (dimensionless) 
Effective average fluid velocity $(\mathrm{m} / \mathrm{s})$

$x \quad$ Axial position along the membrane cylindrical pore (m)

$\varepsilon \quad$ Membrane porosity (dimensionless)

$\Phi \quad$ Steric partition coefficient (dimensionless)

$\lambda \quad$ Ratio of the solute to membrane pore radius (dimensionless)

\section{A2. Transport model for charged ions}

The transport of ions through nanofiltration membranes was modelled by the extended Nernst-Planck equation, originally developed by Bowen et al. (1997) which is written as:

$$
j_{i}=-D_{i, p} \frac{d c_{i}}{d x}-\frac{z_{i} c_{i} D_{i, p}}{R T} F \frac{d \psi}{d x}+K_{i, c} c_{i} J_{v}
$$

where $j_{i}$ is the flux of ion i, which results from the combined effects of diffusion, electronic field gradient and convection (all the symbols are listed in List of Symbols). The diffusivity of ion i $D_{i, p}$ is obtained by $D_{i, p}=K_{i, d} D_{i, \infty}$. The hindrance factors for convection and diffusion, i.e. $K_{i, c}$ and $K_{i, d}$, are calculated based on Appendix A1.

By rearranging Eq. (7), and substituting $j_{i}$ with $J_{v} C_{i, p}$ where $C_{i, p}$ is the ion $\mathrm{i}$ concentration in the permeate, the concentration gradient is expressed as:

$$
\frac{d c_{i}}{d x}=\frac{J_{v}}{D_{i, p}}\left(K_{i, c} c_{i}-C_{i, p}\right)-\frac{z_{i} c_{i}}{R T} F \frac{d \psi}{d x}
$$


By multiplying Eq. (8) with $z_{i}$ and summation over all ions, the potential gradient is obtained as:

$$
\frac{d \psi}{d x}=\frac{\sum_{i=1}^{n} \frac{z_{i} J_{v}}{D_{i, p}}\left(K_{i, c} c_{i}-C_{i, p}\right)}{\frac{F}{R T} \sum_{i=1}^{n} z_{i}^{2} c_{i}}
$$

The electroneutrality inside membrane pores and in the solution is expressed as:

$$
\sum_{i=1}^{n} z_{i} c_{i}=-X, \quad \sum_{i=1}^{n} z_{i} C_{i}=0
$$

where $X$ is the membrane charge density in pores (assume constant throughout the membrane). The $X$ is calculated by $X=\frac{2 \sigma}{r_{p} F}$ (Hagmeyer and Gimbel, 1998) assuming that the charge density in pores equals to the membrane surface charge $\sigma$. The surface charge is calculated assuming that the charge density at the shear plane equals to that at the membrane surface. The $\sigma$ can be expressed by zeta potential $\zeta$ as (Hagmeyer and Gimbel, 1998):

$$
\sigma=\left\{2 \varepsilon_{o} \varepsilon_{b} k T \sum_{i=1}^{n} C_{i, f} N_{A}\left[\exp \left(-\frac{z_{i} e \zeta}{k T}\right)-1\right]\right\}^{0.5}
$$

The partitioning of ion $i$ at the interfaces between the membrane and the bulk solution can be expressed as a combination of the steric, Donnan and solvation effects (Bowen and Welfoot, 2002; Schäfer et al., 2005):

$$
\frac{c_{i}}{C_{i}}=\Phi_{i} \exp \left(-\frac{z_{i} F}{R T} \Delta \psi_{D}\right) \exp \left(-\frac{\Delta W_{i}}{k T}\right)
$$


where the solvation energy barrier $\Delta W_{i}$ can be obtained by the Born model:

$$
\Delta W_{i}=\frac{z_{i}^{2} e^{2}}{8 \pi \varepsilon_{o} r_{i}}\left(\frac{1}{\varepsilon_{p}}-\frac{1}{\varepsilon_{b}}\right)
$$

The dielectric constant of the pores $\varepsilon_{p}$ can be estimated by the average of dielectric constants of one layer of oriented water molecules at the pore walls and an inner center part (Oatley et al., 2013):

$$
\varepsilon_{p}=80-2\left(80-\varepsilon^{*}\right)\left(\frac{d}{r_{p}}\right)+\left(80-\varepsilon^{*}\right)\left(\frac{d}{r_{p}}\right)^{2}
$$

where $\varepsilon^{*}$ is the dielectric constant of one layer of oriented water molecules (a fitting parameter, $\varepsilon^{*} \sim 44.4$ for both NF90 and NF270 based on the best-fit criteria).

Eq. (8) and (9) are solved with the finite difference linearization method proposed by (Schäfer et al. (2005)) with the boundary conditions:

$$
x=0, C_{i}=C_{i, f} ; x=\Delta x, C_{i}=C_{i, p}
$$

List of Symbols

$$
\begin{aligned}
& c_{i} \quad \text { Concentration of ion } \mathrm{i} \text { in membrane pores }\left(\mathrm{mol} / \mathrm{m}^{3}\right) \\
& C_{i} \quad \text { Concentration of ion } \mathrm{i} \text { in the bulk solution }\left(\mathrm{mol} / \mathrm{m}^{3}\right) \\
& C_{i, f} \quad \text { Concentration of ion } \mathrm{i} \text { in the feed }\left(\mathrm{mol} / \mathrm{m}^{3}\right) \\
& C_{i, p}
\end{aligned}
$$




\begin{tabular}{|c|c|}
\hline$d$ & Thickness of the oriented solvent layer (m) \\
\hline$D_{i, p}$ & Hindered diffusivity of ion $\mathrm{i}\left(\mathrm{m}^{2} / \mathrm{s}\right)$ \\
\hline$D_{i, \infty}$ & Bulk diffusivity of ion $\mathrm{i}\left(\mathrm{m}^{2} / \mathrm{s}\right)$ \\
\hline$e$ & Electronic charge $(\mathrm{C})$ \\
\hline$F$ & Faraday constant (C/mol) \\
\hline$j_{i}$ & Flux of ion i $\left(\mathrm{mol} \cdot \mathrm{m}^{-2} / \mathrm{s}\right)$ \\
\hline$J_{v}$ & Permeate water flux $(\mathrm{m} / \mathrm{s})$ \\
\hline$k$ & Boltzmann constant $(\mathrm{J} / \mathrm{k})$ \\
\hline$K_{i, c}$ & Hindered factor for convention of ion i (dimensionless) \\
\hline$K_{i, d}$ & Hindered factor for diffusion of ion i (dimensionless) \\
\hline$r_{i}$ & Radius of ion i (m) \\
\hline$r_{p}$ & Membrane pore radius (m) \\
\hline$R$ & Gas constant $\left(\mathrm{J} \cdot \mathrm{mol}^{-1} \cdot \mathrm{K}^{-1}\right)$ \\
\hline$T$ & Absolute temperature $(\mathrm{K})$ \\
\hline$\Delta W_{i}$ & Solvation energy barrier of ion $\mathrm{i}\left(\mathrm{C}^{2} / \mathrm{F}\right)$ \\
\hline$x$ & Axial position along the membrane cylindrical pore $(\mathrm{m})$ \\
\hline$\Delta x$ & Effective membrane thickness (m) \\
\hline$X$ & Effective membrane charge $\left(\mathrm{mol} / \mathrm{m}^{3}\right)$ \\
\hline$z_{i}$ & Valence of ion i (dimensionless) \\
\hline$\varepsilon_{o}$ & Vacuum dielectric constant $(\mathrm{F} / \mathrm{m})$ \\
\hline
\end{tabular}




$\begin{array}{ll}\varepsilon_{b} & \text { Dielectric constant of bulk solution (dimensionless) } \\ \varepsilon_{p} & \text { Dielectric constant of membrane pores (dimensionless) } \\ \varepsilon^{*} & \text { Dielectric constant of the oriented water layer (dimensionless) } \\ \sigma & \text { Surface charge }\left(\mathrm{C} / \mathrm{m}^{2}\right) \\ \psi & \text { Electric potential (V) } \\ \Delta \psi_{D} & \text { Donnan potential (V) } \\ \zeta & \text { Zeta potential (V) } \\ \Phi_{i} & \text { Steric partition coefficient of ion i (dimensionless) }\end{array}$




\section{References}

Acero, J.L., Rodriguez, E., Meriluoto, J., 2005. Kinetics of reactions between chlorine and the cyanobacterial toxins microcystins. Water Research 39 (8), 1628-1638.

Afifi, M.Z., Blatchley Iii, E.R., 2015. Seasonal dynamics of water and air chemistry in an indoor chlorinated swimming pool. Water Research 68, 771-783.

Aggazzotti, G., Righi, E., Fantuzzi, G., Biasotti, B., Ravera, G., Kanitz, S., Barbone, F., Sansebastiano, G., Battaglia, M.A., Leoni, V., Fabiani, L., Triassi, M., Sciacca, S., 2004. Chlorination by-products (CBPs) in drinking water and adverse pregnancy outcomes in Italy. J Water Health 2 (4), 233-247.

Agus, E., Sedlak, D.L., 2010. Formation and fate of chlorination by-products in reverse osmosis desalination systems. Water Research 44 (5), 1616-1626.

Ahn, W.-Y., Kalinichev, A.G., Clark, M.M., 2008. Effects of background cations on the fouling of polyethersulfone membranes by natural organic matter: experimental and molecular modeling study. Journal of Membrane Science 309 (1-2), 128-140.

Alcudia-León, M.C., Lucena, R., Cárdenas, S., Valcárcel, M., 2013. Determination of parabens in waters by magnetically confined hydrophobic nanoparticle microextraction coupled to gas chromatography/mass spectrometry. Microchemical Journal 110, 643-648.

Ang, W.S., Elimelech, M., 2008. Fatty acid fouling of reverse osmosis membranes: Implications for wastewater reclamation. Water Research 42 (16), 4393-4403.

ANSES, 2012. Health Risk Assessment in swimming pools. Part 1: regulated pools.

ANSES, 2014. Health Risk Assessment in swimming pools. Part 2: Whirlpool.

Aprea, M.-C., Banchi, B., Lunghini, L., Pagliantini, M., Peruzzi, A., Sciarra, G., 2010. Disinfection of swimming pools with chlorine and derivatives: formation of organochlorinated and organobrominated compounds and exposure of pool personnel and swimmers. Natural Science 2 (02), 68.

Arnaud, C.H., 2016. The chemical reactions taking place in your swimming pool. Chemical \& Engineering News 94 (31), 28-32.

ASHRAE, R., 2011. ASHRAE handbook HVAC applications. SI edition. American Society of Heating, Refrigerating and Air-Conditioning Engineers, Inc.

Askins, A., 2013. Cyanuric Acid in Commercial Swimming Pools and its Effects on Chlorine's "Staying Power"And Oxidation Reduction Potentials. Master thesis.

Australia, 2011. AUSTRALIAN DRINKING WATER GUIDELINES 6,National Water Quality Management Strategy.

Bader, E.L., Hrudey, S.E., Froese, K.L., 2004. Urinary excretion half life of trichloroacetic acid as a biomarker of exposure to chlorinated drinking water disinfection by-products. Occupational and environmental medicine 61 (8), 715-716.

Balmer, M.E., Buser, H.-R., Müller, M.D., Poiger, T., 2005. Occurrence of some organic UV filters in wastewater, in surface waters, and in fish from Swiss lakes. Environmental Science \& Technology 39 (4), 953-962. 
Bandini, S., Bruni, L. (2010) Comprehensive Membrane Science and Engineering. Giorno, L. (ed), pp. 67-89, Elsevier, Oxford.

Barbot, E., Moulin, P., 2008. Swimming pool water treatment by ultrafiltrationadsorption process. Journal of Membrane Science 314 (1-2), 50-57.

Bartels, C., Franks, R., Rybar, S., Schierach, M., Wilf, M., 2005. The effect of feed ionic strength on salt passage through reverse osmosis membranes. Desalination 184 (1-3), 185-195.

Bayless, W., Andrews, R.C., 2008. Biodegradation of six haloacetic acids in drinking water. Journal of water and health 6 (1), 15-22.

Beach, H., 1971. Composition and concentrative properties of human urine.

Bernard, A., Nickmilder, M., Voisin, C., 2008. Outdoor swimming pools and the risks of asthma and allergies during adolescence. European respiratory journal 32 (4), 979-988.

Bhattacharyya, L., Rohrer, J.S., 2012. Appendix 2: Dissociation Constants (pKa) of Common Sugars and Alcohols. Applications of Ion Chromatography for Pharmaceutical and Biological Products, 455-456.

Blatchley III, E.R., Cheng, M., 2010. Reaction mechanism for chlorination of urea. Environmental Science \& Technology 44 (22), 8529-8534.

Borges, E.P., Lavorante, A.F., Reis, B.F.d., 2005. Determination of bromide ions in seawater using flow system with chemiluminescence detection. Analytica Chimica Acta 528 (1), 115-119.

Bottoni, P., Bonadonna, L., Chirico, M., Caroli, S., Záray, G., 2014. Emerging issues on degradation by-products deriving from personal care products and pharmaceuticals during disinfection processes of water used in swimming pools. Microchemical Journal 112, 13-16.

Bougeard, C.M.M., Janmohamed, I.H.S., Goslan, E.H., Jefferson, B., Watson, J.S., Morgan, G.H., Parsons, S.A. (2008) Disinfection By-Products in Drinking Water, pp. 95-108, American Chemical Society.

Bowen, W.R., Mohammad, A.W., Hilal, N., 1997. Characterisation of nanofiltration membranes for predictive purposes - use of salts, uncharged solutes and atomic force microscopy. Journal of Membrane Science 126 (1), 91-105.

Bowen, W.R., Welfoot, J.S., 2002. Modelling the performance of membrane nanofiltration-critical assessment and model development. Chemical Engineering Science 57 (7), 1121-1137.

Buczkowska-Radlinska, J., Lagocka, R., Kaczmarek, W., Gorski, M., Nowicka, A., 2013. Prevalence of dental erosion in adolescent competitive swimmers exposed to gas-chlorinated swimming pool water. Clinical Oral Investigations 17 (2), 579-583.

Cammann, K., Hübner, K., 1993. False results in headspace-gas chromatographic analysis of trihalomethanes in swimming pool water due to elevated headspace temperatures. Journal of Chromatography A 648 (1), 294-298.

Cammann, K., Hübner, K., 1995. Trihalomethane concentrations in swimmers' and bath attendants' blood and urine after swimming or working in indoor swimming pools. Archives of Environmental Health: An International Journal 50 (1), 61-65. 
Canada, 2014. Guidelines for Canadian Drinking Water Quality. Federal-ProvincialTerritorial Committee on Drinking Water of the Federal-ProvincialTerritorial Committee on Health and the Environment.

Canada, 2016. Regulation Respecting Water Quality in Swimming Pools and Other Artificial Pools. Environment Quality Act. http://legisquebec.gouv.qc.ca/en/ShowDoc/cr/Q-2,\%20r.\%2039.

Cardador, M., Gallego, M., 2011. Haloacetic acids in swimming pools: swimmer and worker exposure. Environmental Science \& Technology 45 (13), 5783-5790.

Cardador, M.J., Gallego, M., 2010. Determination of haloacetic acids in human urine by headspace gas chromatography-mass spectrometry. J Chromatogr B Analyt Technol Biomed Life Sci 878 (21), 1824-1830.

Caro, J., Gallego, M., 2007. Assessment of Exposure of Workers and Swimmers to Trihalomethanes in an Indoor Swimming Pool. Environmental Science \& Technology 41 (13), 4793-4798.

Caro, J., Gallego, M., 2008. Alveolar Air and Urine Analyses As Biomarkers of Exposure to Trihalomethanes in an Indoor Swimming Pool. Environmental Science \& Technology 42 (13), 5002-5007.

Caro, J., Serrano, A., Gallego, M., 2007. Sensitive headspace gas chromatographymass spectrometry determination of trihalomethanes in urine. Journal of Chromatography B 848 (2), 277-282.

Cassan, D., Mercier, B., Castex, F., Rambaud, A., 2006. Effects of medium-pressure UV lamps radiation on water quality in a chlorinated indoor swimming pool. Chemosphere 62 (9), 1507-1513.

Catto, C., Sabrina, S., Ginette, C.-T., Manuel, R., Robert, T., 2012. Occurrence and Spatial and Temporal Variations of Disinfection By-Products in the Water and Air of Two Indoor Swimming Pools. Int J Environ Res Public Health 9 (8), 2562-2586.

Chalatip, R., Chawalit, R., Nopawan, R., 2009. Removal of haloacetic acids by nanofiltration. Journal of Environmental Sciences 21 (1), 96-100.

Chellam, S., 2000. Effects of Nanofiltration on Trihalomethane and Haloacetic Acid Precursor Removal and Speciation in Waters Containing Low Concentrations of Bromide Ion. Environmental Science \& Technology 34 (9), 1813-1820.

Chen, B., Lee, W., Westerhoff, P.K., Krasner, S.W., Herckes, P., 2010. Solar photolysis kinetics of disinfection byproducts. Water Research 44 (11), 34013409.

Chen, M.J., Lin, C.H., Duh, J.M., Chou, W.S., Hsu, H.T., 2011. Development of a multi-pathway probabilistic health risk assessment model for swimmers exposed to chloroform in indoor swimming pools. J Hazard Mater 185 (2-3), 1037-1044.

Chen, S.-S., Taylor, J.S., Mulford, L.A., Norris, C.D., 2004. Influences of molecular weight, molecular size, flux, and recovery for aromatic pesticide removal by nanofiltration membranes. Desalination 160 (2), 103-111.

Childress, A.E., Elimelech, M., 1996. Effect of solution chemistry on the surface charge of polymeric reverse osmosis and nanofiltration membranes. Journal of Membrane Science 119 (2), 253-268. 
Childress, A.E., Elimelech, M., 2000. Relating nanofiltration membrane performance to membrane charge (electrokinetic) characteristics. Environmental Science \& Technology 34 (17), 3710-3716.

Chin, A., Bérubé, P.R., 2005. Removal of disinfection by-product precursors with ozone-UV advanced oxidation process. Water Research 39 (10), 2136-2144.

China, 1996. Hygienic standard for swimming place (GB 9667-1996).

Chowdhury, S., 2015. Predicting human exposure and risk from chlorinated indoor swimming pool: a case study. Environmental Monitoring and Assessment 187 (8), 502-502.

Chowdhury, S., Alhooshani, K., Karanfil, T., 2014. Disinfection byproducts in swimming pool: Occurrences, implications and future needs. Water Research 53, 68-109.

Chu, H., Nieuwenhuijsen, M., 2002. Distribution and determinants of trihalomethane concentrations in indoor swimming pools. Occupational and environmental medicine 59 (4), 243-247.

Chuang, Y.H., Wang, G.S., Tung, H.H., 2011. Chlorine residuals and haloacetic acid reduction in rapid sand filtration. Chemosphere 85 (7), 1146-1153.

Chun, M.-S., Lee, S.-Y., Yang, S.-M., 2003. Estimation of zeta potential by electrokinetic analysis of ionic fluid flows through a divergent microchannel. Journal of Colloid and Interface Science 266 (1), 120-126.

Colorado, 1998. State Board Of Health Regulations Pertaining to Swimming Pools and Mineral Baths. Colorado Depatrment of Public Health and Environment. Water Quality Control Division.

Craun, G., Calderon, R., Craun, M., 2005. Outbreaks associated with recreational water in the United States. International journal of environmental health research 15 (4), 243-262.

Croue, J.P., Reckhow, D.A., 1989. Destruction of chlorination byproducts with sulfite. Environmental Science \& Technology 23 (11), 1412-1419.

Dad, A., Jeong, C.H., Pals, J.A., Wagner, E.D., Plewa, M.J., 2013. Pyruvate remediation of cell stress and genotoxicity induced by haloacetic acid drinking water disinfection by - products. Environmental and molecular mutagenesis 54 (8), 629-637.

De Laat, J., Feng, W., Freyfer, D.A., Dossier-Berne, F., 2011. Concentration levels of urea in swimming pool water and reactivity of chlorine with urea. Water Res 45 (3), 1139-1146.

Deen, W., 1987. Hindered transport of large molecules in liquid - filled pores. AIChE Journal 33 (9), 1409-1425.

Directive, E., 1998. EU Directive 98/83/EC of 3 November

Do, V.T., Reinhard, M., Tang, C.Y., Leckie, J.O., 2012a. Effects of hypochlorous acid exposure on the rejection of salt, polyethylene glycols, boron and $\operatorname{arsenic}(\mathrm{V})$ by nanofiltration and reverse osmosis membranes. Water Research 46 (16), 5217-5223.

Do, V.T., Tang, C.Y., Reinhard, M., Leckie, J.O., 2012b. Degradation of polyamide nanofiltration and reverse osmosis membranes by hypochlorite. Environmental Science \& Technology 46 (2), 852-859. 
Do, V.T., Tang, C.Y., Reinhard, M., Leckie, J.O., 2012c. Effects of chlorine exposure conditions on physiochemical properties and performance of a polyamide membrane-mechanisms and implications. Environmental Science \& Technology 46 (24), 13184-13192.

Doederer, K., Farré, M.J., Pidou, M., Weinberg, H.S., Gernjak, W., 2014. Rejection of disinfection by-products by RO and NF membranes: influence of solute properties and operational parameters. Journal of Membrane Science 467, 195-205.

Domino, M., Pepich, B., Munch, D., Xie, Y., 2003. EPA method 552.3, revision 1.0, determination of haloacetic acids and dalapon in drinking water by liquidliquid extraction, derivatization and gas chromatography with electron capture detection. EPA document EPA-815-B-03-002. US Environmental Protection Agency, Cincinnati.

Dong, L.-X., Huang, X.-c., Wang, Z., Yang, Z., Wang, X.-m., Tang, C.Y., 2016. A thin-film nanocomposite nanofiltration membrane prepared on a support with in situ embedded zeolite nanoparticles. Separation and Purification Technology 166, 230-239.

Dyck, R., Sadiq, R., Rodriguez, M.J., Simard, S., Tardif, R., 2011. Trihalomethane exposures in indoor swimming pools: a level III fugacity model. Water Res 45 (16), 5084-5098.

Einstein, A., 1956. Investigations on the Theory of the Brownian Movement.

EPA, 2005. Drinking Water Criteria Document For Brominated Trihalomethanes, EPA Office of Water, Washington, D.C., EPA-822-R-05-011, November 15.

EPA, U., 1979. National interim primary drinking water regulations: control of trihalomethanes in drinking water: final rules. 44, 68624-68705.

EPA, U., 1998. National primary drinking water regulations: disinfectants and disinfection byproducts; final rule. 63, 69390-69476.

Erdinger, L., Kühn, K.P., Kirsch, F., Feldhues, R., Fröbel, T., Nohynek, B., Gabrio, T., 2004. Pathways of trihalomethane uptake in swimming pools. Int J Hyg Environ Health 207 (6), 571-575.

Erdinger, L., Kirsch, F., Sonntag, H.G., 1998. Irritating effects of disinfection byproducts in swimming pools. Zentralblatt Fur Hygiene Und Umweltmedizin 200 (5-6), 491-503.

Fabris, R., Chow, C.W.K., Drikas, M., Eikebrokk, B., 2008. Comparison of NOM character in selected Australian and Norwegian drinking waters. Water Research 42 (15), 4188-4196.

Fan, L., Harris, J.L., Roddick, F.A., Booker, N.A., 2001. Influence of the characteristics of natural organic matter on the fouling of microfiltration membranes. Water Research 35 (18), 4455-4463.

Fang, J.-Y., Ling, L., Shang, C., 2013. Kinetics and mechanisms of pH-dependent degradation of halonitromethanes by UV photolysis. Water Research 47 (3), 1257-1266.

Fantuzzi, G., Righi, E., Predieri, G., Ceppelli, G., Gobba, F., Aggazzotti, G., 2001. Occupational exposure to trihalomethanes in indoor swimming pools. Science of the Total Environment 264 (3), 257-265. 
Fantuzzi, G., Righi, E., Predieri, G., Giacobazzi, P., Mastroianni, K., Aggazzotti, G., 2010. Prevalence of Ocular, Respiratory and Cutaneous Symptoms in Indoor Swimming Pool Workers and Exposure to Disinfection By-Products (DBPs). Int J Environ Res Public Health 7 (4), 1379-1391.

Feng, Y., Smith, D.W., Bolton, J.R., 2007. Photolysis of aqueous free chlorine species ( $\mathrm{HOCl}$ and $\mathrm{OCl}$ ) with $254 \mathrm{~nm}$ ultraviolet light. Journal of Environmental Engineering and Science 6 (3), 277-284.

Ferry, J.D., 1936. Ultrafilter Membranes and Ultrafiltration. Chemical Reviews 18 (3), 373-455.

Fischer, K., Fries, E., Körner, W., Schmalz, C., Zwiener, C., 2012. New developments in the trace analysis of organic water pollutants. Applied Microbiology and Biotechnology 94 (1), 11-28.

Florentin, A., Hautemanière, A., Hartemann, P., 2011. Health effects of disinfection by-products in chlorinated swimming pools. Int J Hyg Environ Health 214 (6), 461-469.

Florida, 2009. PUBLIC SWIMMING POOLS AND BATHING PLACES (CHAPTER 64E-9). Florida Administrative Code \& Florida Administrative Register. https://www.flrules.org/gateway/ChapterHome.asp?Chapter=64e$\underline{9}$.

Font-Ribera, L., Kogevinas, M., Schmalz, C., Zwiener, C., Marco, E., Grimalt, J.O., Liu, J., Zhang, X., Mitch, W., Critelli, R., 2016. Environmental and personal determinants of the uptake of disinfection by-products during swimming. Environmental research 149, 206-215.

Fujioka, T., Khan, S.J., Poussade, Y., Drewes, J.E., Nghiem, L.D., 2012. Nnitrosamine removal by reverse osmosis for indirect potable water reuse $-\mathrm{A}$ critical review based on observations from laboratory-, pilot- and full-scale studies. Separation and Purification Technology 98, 503-515.

Geankoplis, C.J., 1993. Transport Processes and Unit Operations. p400-401. Prentice-Hall International, Inc, 393-397.

Georgia, 2013. Rules and Regulations. Swimming Pools, Spa and Recreational Water Parks, Chapter 511-3-5. Georgia Department of Public Health. http://pooloperationmanagement.com/about/locations/georgia/.

Germany, 2012. Treatment of the water of swimming pools and baths - Part 1: General requirements, Standard DIN 19643-1: 2012-11.

Giller, S., Le Curieux, F., Erb, F., Marzin, D., 1997. Comparative genotoxicity of halogenated acetic acids found in drinking water. Mutagenesis 12 (5), 321328.

Ginn, S.T., Conley, J.C., Sergent, R.H., Fellers, B.D., 1989. Bromine Biocides in Alkaline and High Demand Cooling Waters, Paper Number 157, Corrosion '89, NACE (Original publisher and copyright holder).

Glauner, T., Kunz, F., Zwiener, C., Frimmel, F., 2005a. Elimination of Swimming Pool Water Disinfection By-products with Advanced Oxidation Processes (AOPs). Acta Hydrochimica et Hydrobiologica 33 (6), 585-594.

Glauner, T., Waldmann, P., Frimmel, F.H., Zwiener, C., 2005b. Swimming pool water-fractionation and genotoxicological characterization of organic constituents. Water Res 39 (18), 4494-4502. 
Goodman, M., Hays, S., 2008. Asthma and Swimming: A Meta-Analysis. Journal of Asthma 45 (8), 639-647.

Government, Q., 2004. Queensland Health Swimming and Spa Pool Water Quality and Operational Guidelines. p10.

Greyshock, A.E., Vikesland, P.J., 2006. Triclosan Reactivity in Chloraminated Waters. Environmental Science \& Technology 40 (8), 2615-2622.

Grigorescu, A.S., Lapara, T.M., Hozalski, R.M., 2010. Biodegradation of haloacetic acids and potential applicability to drinking water treatment. Romanian Journal of Biochemistry 47 (2), 165-177.

Guillen, G.R., Hoek, E.M., 2010. Development and Testing of "Smart" Nanofiltration Membranes, NWRI Final Project Report. Los Angeles, CA: Department of Civil \& Environmental Engineering, University of California.

Hagmeyer, G., Gimbel, R., 1998. Modelling the salt rejection of nanofiltration membranes for ternary ion mixtures and for single salts at different $\mathrm{pH}$ values. Desalination 117 (1-3), 247-256.

Hamidin, N., Yu, Q.J., Connell, D.W., 2008. Human health risk assessment of chlorinated disinfection by-products in drinking water using a probabilistic approach. Water Res 42 (13), 3263-3274.

Hansen, K.M., Albrechtsen, H.-J., Andersen, H.R., 2013. Optimal pH in chlorinated swimming pools-balancing formation of by-products. J Water Health 11 (3), 465-472.

Hansen, K.M., Willach, S., Antoniou, M.G., Mosbaek, H., Albrechtsen, H.J., Andersen, H.R., 2012a. Effect of $\mathrm{pH}$ on the formation of disinfection byproducts in swimming pool water--is less THM better? Water Res 46 (19), 6399-6409.

Hansen, K.M., Willach, S., Mosbaek, H., Andersen, H.R., 2012b. Particles in swimming pool filters--does $\mathrm{pH}$ determine the DBP formation? Chemosphere 87 (3), 241-247.

Heimburg, T., 2008. Thermal biophysics of membranes. John Wiley \& Sons.

Herzberg, M., Kang, S., Elimelech, M., 2009. Role of extracellular polymeric substances (EPS) in biofouling of reverse osmosis membranes. Environmental Science \& Technology 43 (12), 4393-4398.

Hoffman, C.S., Mendola, P., Savitz, D.A., Herring, A.H., Loomis, D., Hartmann, K.E., Singer, P.C., Weinberg, H.S., Olshan, A.F., 2008. Drinking water disinfection by-product exposure and fetal growth. Epidemiology 19 (5), 729-737.

Hong, S., Elimelech, M., 1997. Chemical and physical aspects of natural organic matter (NOM) fouling of nanofiltration membranes. Journal of Membrane Science 132 (2), 159-181.

Hozalski, R.M., Zhang, L., Arnold, W.A., 2001. Reduction of haloacetic acids by Fe-0: Implications for treatment and fate. Environmental Science \& Technology 35 (11), 2258-2263.

Hrudey, S.E. (2008) Chlorination Disinfection By-Products (DBPs) in Drinking Water and Public Health in Canada: A Primer for Public Health Practitioners: Reviewing Evidence from Over 30 Years of Research: A Knowledge Translation Review, Citeseer. 
Hrudey, S.E., Backer, L.C., Humpage, A.R., Krasner, S.W., Michaud, D.S., Moore, L.E., Singer, P.C., Stanford, B.D., 2015. Evaluating Evidence for Association of Human Bladder Cancer with Drinking-Water Chlorination Disinfection By-Products. Journal of Toxicology and Environmental Health, Part B 18 (5), 213-241.

Hua, G., Reckhow, D.A., 2008. DBP formation during chlorination and chloramination: effect of reaction time, $\mathrm{pH}$, dosage, and temperature. Journal (American Water Works Association) 100 (8), 82-95.

Hua, G., Reckhow, D.A., Kim, J., 2006. Effect of Bromide and Iodide Ions on the Formation and Speciation of Disinfection Byproducts during Chlorination. Environmental Science \& Technology 40 (9), 3050-3056.

IARC (2004) IARC Working Group on the Evaluation of Carcinogenic Risks to Humans. Some drinking-water disinfectants and contaminants, including arsenic. World Health Organization. International Agency for Research on Cancer.

Jin, X., Huang, X., Hoek, E.M., 2009. Role of specific ion interactions in seawater RO membrane fouling by alginic acid. Environmental Science \& Technology 43 (10), 3580-3587.

Jo, C.H., Dietrich, A.M., Tanko, J.M., 2011. Simultaneous degradation of disinfection byproducts and earthy-musty odorants by the UV/H2O2 advanced oxidation process. Water Research 45 (8), 2507-2516.

Jones, C.E., Carpenter, L.J., 2005. Solar Photolysis of CH2I2, CH2ICl, and CH2IBr in Water, Saltwater, and Seawater. Environmental Science \& Technology 39 (16), 6130-6137.

Judd, S.J., Black, S.H., 2000. Disinfection by-product formation in swimming pool waters: a simple mass balance. Water Research 34 (5), 1611-1619.

Judd, S.J., Bullock, G., 2003. The fate of chlorine and organic materials in swimming pools. Chemosphere 51 (9), 869-879.

Kamal, H., Abd-Elrahim, F.M., Lotfy, S., 2014. Characterization and some properties of cellulose acetate-co-polyethylene oxide blends prepared by the use of gamma irradiation. Journal of Radiation Research and Applied Sciences 7 (2), 146-153.

Kanan, A., 2010. Occurrence and formation of disinfection by-products in indoor swimming pools water. PhD thesis.

Kanan, A., Karanfil, T., 2011. Formation of disinfection by-products in indoor swimming pool water: the contribution from filling water natural organic matter and swimmer body fluids. Water Res 45 (2), 926-932.

Kanan, A., Selbes, M., Karanfil, T. (2015) Recent Advances in Disinfection byProducts. Karanfil, T., Mitch, B., Westerhoff, P. and Xie, Y. (eds), pp. 405430, Amer Chemical Soc, Washington.

Kargalioglu, Y., McMillan, B.J., Minear, R.A., Plewa, M.J., 2002. Analysis of the cytotoxicity and mutagenicity of drinking water disinfection by - products in Salmonella typhimurium. Teratogenesis, carcinogenesis, and mutagenesis 22 (2), 113-128. 
Keuten, M.G., Schets, F.M., Schijven, J.F., Verberk, J.Q., van Dijk, J.C., 2012. Definition and quantification of initial anthropogenic pollutant release in swimming pools. Water Res 46 (11), 3682-3692.

Keuten, M.G.A., Peters, M.C.F.M., Daanen, H.A.M., de Kreuk, M.K., Rietveld, L.C., van Dijk, J.C., 2014. Quantification of continual anthropogenic pollutants released in swimming pools. Water Research 53, 259-270.

Kim, H., Haltmeier, P., Klotz, J.B., Weisel, C.P., 1999. Evaluation of Biomarkers of Environmental Exposures: Urinary Haloacetic Acids Associated with Ingestion of Chlorinated Drinking Water. Environmental research 80 (2), 187-195.

Kim, H., Shim, J., Lee, S., 2002. Formation of disinfection by-products in chlorinated swimming pool water. Chemosphere 46 (1), 123-130.

Kimura, K., Amy, G., Drewes, J.E., Heberer, T., Kim, T.-U., Watanabe, Y., 2003. Rejection of organic micropollutants (disinfection by-products, endocrine disrupting compounds, and pharmaceutically active compounds) by NF/RO membranes. Journal of Membrane Science 227 (1), 113-121.

Kiso, Y., Muroshige, K., Oguchi, T., Yamada, T., Hhirose, M., Ohara, T., Shintani, T., 2010. Effect of molecular shape on rejection of uncharged organic compounds by nanofiltration membranes and on calculated pore radii. Journal of Membrane Science 358 (1-2), 101-113.

Klüpfel, A.M., Glauner, T., Zwiener, C., Frimmel, F.H., 2011. Nanofiltration for enhanced removal of disinfection by-product (DBP) precursors in swimming pool water-retention and water quality estimation. Water Science and Technology 63 (8), 1716-1725.

Kogevinas, M., Villanueva, C., Font Ribera, L., Liviac, D., Bustamante, M., Espinoza, F., Nieuwenhuijsen, M., Espinosa, A., Fernandez, P., DeMarini, D., Grimalt, J., Grummt, T., Marcos, R., 2010. Genotoxic Effects in Swimmers Exposed to Disinfection By-products in Indoor Swimming Pools. Environmental Health Perspectives 118 (11), 1531-1537.

Kong, F.-X., Yang, H.-w., Wang, X.-m., Xie, Y.F., 2014. Rejection of nine haloacetic acids and coupled reverse draw solute permeation in forward osmosis. Desalination 341, 1-9.

Korshin, G.V., Jensen, M.D., 2001. Electrochemical reduction of haloacetic acids and exploration of their removal by electrochemical treatment. Electrochimica Acta 47 (5), 747-751.

Krasner, S.W., Weinberg, H.S., Richardson, S.D., Pastor, S.J., Chinn, R., Sclimenti, M.J., Onstad, G.D., Thruston, A.D., 2006. Occurrence of a New Generation of Disinfection Byproducts $\uparrow$. Environ Sci Technol 40 (23), 7175-7185.

Kwok, W.M., Zhao, C., Li, Y.-L., Guan, X., Wang, D., Phillips, D.L., 2004. WaterCatalyzed Dehalogenation Reactions of Isobromoform and Its Reaction Products. Journal of the American Chemical Society 126 (10), 3119-3131.

López-Muñoz, M.J., Sotto, A., Arsuaga, J.M., Van der Bruggen, B., 2009. Influence of membrane, solute and solution properties on the retention of phenolic compounds in aqueous solution by nanofiltration membranes. Separation and Purification Technology 66 (1), 194-201. 
Lévesque, B., Ayotte, P., Tardif, R., Charest-Tardif, G., 2000. Evaluation of the health risk associated with exposure to chloroform in indoor swimming pools. Journal of toxicology and environmental health Part A 61 (4), 225-243.

Lambropoulou, D.A., Giokas, D.L., Sakkas, V.A., Albanis, T.A., Karayannis, M.I., 2002. Gas chromatographic determination of 2-hydroxy-4methoxybenzophenone and octyldimethyl-p-aminobenzoic acid sunscreen agents in swimming pool and bathing waters by solid-phase microextraction. Journal of Chromatography A 967 (2), 243-253.

Lamont Bradford, W., 2014. What Bathers Put Into a Pool: A Critical Review of Body Fluids and a Body Fluid Analog. International Journal of Aquatic Research \& Education 8 (2), 168-181.

Lee, J., Ha, K.T., Zoh, K.D., 2009. Characteristics of trihalomethane (THM) production and associated health risk assessment in swimming pool waters treated with different disinfection methods. Sci Total Environ 407 (6), 19901997.

Lee, J., Jun, M.J., Lee, M.H., Eom, S.W., Zoh, K.D., 2010. Production of various disinfection byproducts in indoor swimming pool waters treated with different disinfection methods. Int J Hyg Environ Health 213 (6), 465-474.

Li, C., Yang, Y.J., Yu, J., Zhang, T.-q., Mao, X., Shao, W., 2012. Second-Order Chlorine Decay and Trihalomethanes Formation in a Pilot-Scale Water Distribution Systems. Water Environment Research 84 (8), 656-661.

Li, J., Blatchley, E.R., 2007. Volatile disinfection byproduct formation resulting from chlorination of organic-nitrogen precursors in swimming pools. Environmental Science \& Technology 41 (19), 6732-6739.

Lian, L., Li, J., Blatchley III, E.R., 2014. Volatile disinfection byproducts resulting from chlorination of uric acid: implications for swimming pools. Environmental Science \& Technology 48 (6), 3210-3217.

Lifongo, L.L., Bowden, D.J., Brimblecombe, P., 2004. Photodegradation of haloacetic acids in water. Chemosphere 55 (3), 467-476.

Lifongo, L.L., Bowden, D.J., Brimblecombe, P., 2010. Thermal degradation of haloacetic acids in water. International Journal of the Physical Sciences 5 (6), 738-747.

Liu, W., Cheung, L.-M., Yang, X., Shang, C., 2006. THM, HAA and CNCl formation from UV irradiation and chlor(am)ination of selected organic waters. Water Research 40 (10), 2033-2043.

Liviac, D., Wagner, E.D., Mitch, W.A., Altonji, M.J., Plewa, M.J., 2010. Genotoxicity of water concentrates from recreational pools after various disinfection methods. Environ Sci Technol 44 (9), 3527-3532.

Loos, R., Barceló, D., 2001. Determination of haloacetic acids in aqueous environments by solid-phase extraction followed by ion-pair liquid chromatography-electrospray ionization mass spectrometric detection. Journal of Chromatography A 938 (1-2), 45-55.

Lourencetti, C., Grimalt, J.O., Marco, E., Fernandez, P., Font-Ribera, L., Villanueva, C.M., Kogevinas, M., 2012. Trihalomethanes in chlorine and bromine disinfected swimming pools: air-water distributions and human exposure. Environment international 45, 59-67. 
Mahlangu, T.O., Hoek, E.M.V., Mamba, B.B., Verliefde, A.R.D., 2014. Influence of organic, colloidal and combined fouling on $\mathrm{NF}$ rejection of $\mathrm{NaCl}$ and carbamazepine: role of solute-foulant-membrane interactions and cakeenhanced concentration polarisation. Journal of Membrane Science 471, 3546.

Manasfi, T., De Méo, M., Coulomb, B., Di Giorgio, C., Boudenne, J.-L., 2016. Identification of disinfection by-products in freshwater and seawater swimming pools and evaluation of genotoxicity. Environment international 88, 94-102.

Marina, L.S., Ibarluzea, J., Basterrechea, M., Goñi, F., Ulibarrena, E., Artieda, J., Orruño, I., 2009. Indoor air and bathing water pollution in indoor swimming pools in Guipuzcoa (Spain). Gaceta Sanitaria 23 (2), 115-120.

Matilainen, A., Sillanpää, M., 2010. Removal of natural organic matter from drinking water by advanced oxidation processes. Chemosphere 80 (4), 351365.

Meike, K., Iris, H., Ocke, R., Carsten K., S., 2009. Haloacetonitriles -another important group of disinfection byproducts in swimming pool water. Swimming Pool \& Spa International Conference, London, March 2009.

Mi, B., Elimelech, M., 2010. Organic fouling of forward osmosis membranes: fouling reversibility and cleaning without chemical reagents. Journal of Membrane Science 348 (1-2), 337-345.

Michalski, R., Mathews, B., 2007. Occurrence of chlorite, chlorate and bromate in disinfected swimming pool water. Polish journal of environmental studies 16 (2), 237-241.

Mo, H., Tay, K., Ng, H., 2008. Fouling of reverse osmosis membrane by protein (BSA): Effects of $\mathrm{pH}$, calcium, magnesium, ionic strength and temperature. Journal of Membrane Science 315 (1-2), 28-35.

Moffa, P.E., Davis, D.P., Somerlot, C., Sharek, D., Gresser, B., Smith, T., 2006. Alternative Disinfection Technology Demonstrates Advantages for Wet Weather Applications-A Pilot Study of Powdered Bromine Technology. Proceedings of the Water Environment Federation 2006 (12), 1202-1218.

Motsa, M.M., Mamba, B.B., D’Haese, A., Hoek, E.M.V., Verliefde, A.R.D., 2014. Organic fouling in forward osmosis membranes: the role of feed solution chemistry and membrane structural properties. Journal of Membrane Science 460, 99-109.

Muellner, M.G., Wagner, E.D., McCalla, K., Richardson, S.D., Woo, Y.-T., Plewa, M.J., 2007a. Haloacetonitriles vs. regulated haloacetic acids: are nitrogencontaining DBPs more toxic? Environmental Science \& Technology 41 (2), 645-651.

Muellner, M.G., Wagner, E.D., McCalla, K., Richardson, S.D., Woo, Y.-T., Plewa, M.J., 2007b. Haloacetonitriles vs. Regulated Haloacetic Acids: Are Nitrogen-Containing DBPs More Toxic? Environmental Science \& Technology 41 (208), 645-651.

Murphy, J.L., Arrowood, M.J., Lu, X., Hlavsa, M.C., Beach, M.J., Hill, V.R., 2015. Effect of cyanuric acid on the inactivation of Cryptosporidium parvum under 
hyperchlorination conditions. Environmental Science \& Technology 49 (12), 7348-7355.

Nakajima, M., Kawakami, T., Niino, T., Takahashi, Y., Onodera, S., 2009. Aquatic Fate of Sunscreen Agents Octyl-4-methoxycinnamate and Octyl-4dimethylaminobenzoate in Model Swimming Pools and the Mutagenic Assays of Their Chlorination Byproducts. Journal of Health Science 55 (3), 363-372.

Nalepa, C.J., 2004. 25 Years Of Bromine Chemistry In Industrial Water Systems: A Review. NACE International 04087., NACE International.

NEA, 2005. Code of Practice on Environmental Health, National Environment Agnecy, Singapore.

NEA, 2008. Environmental Public Health Act. Chapter 95. Environmental Public Health (Quality of Piped Drinking Water) Regulations.

Negreira, N., Canosa, P., Rodríguez, I., Ramil, M., Rubí, E., Cela, R., 2008. Study of some UV filters stability in chlorinated water and identification of halogenated by-products by gas chromatography-mass spectrometry. Journal of Chromatography A 1178 (1-2), 206-214.

Nghiem, L.D., Schäfer, A.I., Elimelech, M., 2004. Removal of natural hormones by nanofiltration membranes: measurement, modeling, and mechanisms. Environmental Science \& Technology 38 (6), 1888-1896.

Nghiem, L.D., Schäfer, A.I., Elimelech, M., 2005. Pharmaceutical retention mechanisms by nanofiltration membranes. Environmental Science \& Technology 39 (19), 7698-7705.

Nieuwenhuijsen, M.J., Toledano, M.B., Elliott, P., 2000. Uptake of chlorination disinfection by-products; a review and a discussion of its implications for exposure assessment in epidemiological studies. Journal of Exposure Analysis and Environmental Epidemiology 10 (6), 586-599.

NSPF, 2006. Certified Pool-Spa Operator Handbook: National Swimming Pool Foundation, Colorado Springs, CO.

Oatley, D.L., Llenas, L., Aljohani, N.H.M., Williams, P.M., Martínez-Lladó, X., Rovira, M., de Pablo, J., 2013. Investigation of the dielectric properties of nanofiltration membranes. Desalination 315, 100-106.

Oo, M.H., Song, L., 2009. Effect of pH and ionic strength on boron removal by RO membranes. Desalination 246 (1-3), 605-612.

Ozaki, H., Li, H., 2002. Rejection of organic compounds by ultra-low pressure reverse osmosis membrane. Water Research 36 (1), 123-130.

Panyakapo, M., Soontornchai, S., Paopuree, P., 2008. Cancer risk assessment from exposure to trihalomethanes in tap water and swimming pool water. Journal of Environmental Sciences 20 (3), 372-378.

Pardo, A., Nevo, K., Vigiser, D., Lazarov, A., 2007. The effect of physical and chemical properties of swimming pool water and its close environment on the development of contact dermatitis in hydrotherapists. American Journal of Industrial Medicine 50 (2), 122-126.

Parinet, J., Tabaries, S., Coulomb, B., Vassalo, L., Boudenne, J.L., 2012. Exposure levels to brominated compounds in seawater swimming pools treated with chlorine. Water Res 46 (3), 828-836. 
Pereira, I., Sá, C.S.A., Boaventura, R.A.R., 2012. Analysis of haloacetic acids in water and air (aerosols) from indoor swimming pools using HSSPME/GC/ECD. Journal of environmental science and health. Part A, Toxic/hazardous substances \& environmental engineering 47 (2), 176-183.

Plewa, M.J., Kargalioglu, Y., Vankerk, D., Minear, R.A., Wagner, E.D., 2002. Mammalian cell cytotoxicity and genotoxicity analysis of drinking water disinfection by - products. Environmental and molecular mutagenesis 40 (2), 134-142.

Plewa, M.J., Simmons, J.E., Richardson, S.D., Wagner, E.D., 2010. Mammalian cell cytotoxicity and genotoxicity of the haloacetic acids, a major class of drinking water disinfection by - products. Environmental and molecular mutagenesis 51 (8 - 9), 871-878.

Plewa, M.J., Wagner, E.D., Muellner, M.G., Hsu, K.-M., Richardson, S.D. (2008) Disinfection By-Products in Drinking Water, pp. 36-50, American Chemical Society.

Plewa, M.J., Wagner, E.D., Richardson, S.D., Thruston, A.D., Woo, Y.-T., McKague, A.B., 2004. Chemical and Biological Characterization of Newly Discovered Iodoacid Drinking Water Disinfection Byproducts. Environmental Science \& Technology 38 (18), 4713-4722.

Pluchon, C., Serodes, J.B., Berthiaume, C., Charette, S.J., Gilbert, Y., Filion, G., Fournier-Larente, J., Rodriguez, M., Duchaine, C., 2013. Haloacetic acid degradation by a biofilm in a simulated drinking water distribution system. Water Science and Technology-Water Supply 13 (2), 447-461.

Procházka, E., Escher, B.I., Plewa, M.J., Leusch, F.D., 2015. In Vitro Cytotoxicity and Adaptive Stress Responses to Selected Haloacetic Acid and Halobenzoquinone Water Disinfection Byproducts. Chemical Research in Toxicology 28 (10), 2059-2068.

Reusch, $\quad$ W., 2013. Carboxylic Acids. https://www2.chemistry.msu.edu/faculty/reusch/virttxtjml/crbacid1.htm.

Richardson, S.D., 2010. Environmental mass spectrometry: emerging contaminants and current issues. Analytical chemistry 82 (12), 4742-4774.

Richardson, S.D. (2011) Encyclopedia of environmental health. Nriagu, J.O. (ed), pp. 110-136, Elsevier, Burlington.

Richardson, S.D., DeMarini, D.M., Kogevinas, M., Fernandez, P., Marco, E., Lourencetti, C., Ballesté, C., Heederik, D., Meliefste, K., McKague, A.B., 2010. What's in the pool? A comprehensive identification of disinfection byproducts and assessment of mutagenicity of chlorinated and brominated swimming pool water.

Richardson, S.D., Plewa, M.J., Wagner, E.D., Schoeny, R., Demarini, D.M., 2007. Occurrence, genotoxicity, and carcinogenicity of regulated and emerging disinfection by-products in drinking water: a review and roadmap for research. Mutation research 636 (1-3), 178-242.

Richardson, S.D., Thruston, A.D., Caughran, T.V., Chen, P.H., Collette, T.W., Floyd, T.L., Schenck, K.M., Lykins, B.W., Sun, G.-r., Majetich, G., 1999. Identification of new ozone disinfection byproducts in drinking water. Environ Sci Technol 33 (19), 3368-3377. 
Rock, J.J., 1974. Formation of haloforms during chlorination of natural water Water Treat. Exam. 23, 234-243.

Rudra, A., Thacker, N., Pande, S., 2005. Hydrogen Peroxide and Ultraviolet Irradiations in Water Treatment. Environmental Monitoring and Assessment 109 (1-3), 189-197.

Rule, K.L., Ebbett, V.R., Vikesland, P.J., 2005. Formation of Chloroform and Chlorinated Organics by Free-Chlorine-Mediated Oxidation of Triclosan. Environmental Science \& Technology 39 (9), 3176-3185.

Sadiq, R., Rodriguez, M.J., 2004. Disinfection by-products (DBPs) in drinking water and predictive models for their occurrence: a review. Science of the Total Environment 321 (1-3), 21-46.

Sakkas, V.A., Giokas, D.L., Lambropoulou, D.A., Albanis, T.A., 2003. Aqueous photolysis of the sunscreen agent octyl-dimethyl-p-aminobenzoic acid: Formation of disinfection byproducts in chlorinated swimming pool water. Journal of Chromatography A 1016 (2), 211-222.

Sarrión, M.N., Santos, F.J., Galceran, M.T., 2000. In Situ Derivatization/Solid-Phase Microextraction for the Determination of Haloacetic Acids in Water. Analytical chemistry 72 (20), 4865-4873.

Schäfer, A.I., Fane, A.G., Waite, T.D. (2005) Nanofiltration: principles and applications, Elsevier.

Schmalz, C., 2012. Formation, phase transfer and toxicity of halogenated disinfection by-products in the processing cycle of swimming pool water.

Schmalz, C., Frimmel, F.H., Zwiener, C., 2011. Trichloramine in swimming poolsformation and mass transfer. Water Research 45 (8), 2681-2690.

Scully, F.E., Howell, G.D., Kravitz, R., Jewell, J.T., Hahn, V., Speed, M., 1988. Proteins in natural waters and their relation to the formation of chlorinated organics during water disinfection. Environ Sci Technol 22 (5), 537-542.

Semião, A.J.C., Schäfer, A.I., 2013. Removal of adsorbing estrogenic micropollutants by nanofiltration membranes. Part A-Experimental evidence. Journal of Membrane Science 431 (0), 244-256.

Sentana, I., Rodriguez, M., Sentana, E., Prats, D., 2010. Effect of pressure and pH over the removal of disinfection by-products using nanofiltration membranes in discontinuous systems. Desalination and Water Treatment 23 (1-3), 3-12.

Serpone, N., Salinaro, A., Emeline, A.V., Horikoshi, S., Hidaka, H., Zhao, J., 2002. An in vitro systematic spectroscopic examination of the photostabilities of a random set of commercial sunscreen lotions and their chemical UVB/UVA active agents. Photochemical \& Photobiological Sciences 1 (12), 970-981.

Shah, A.D., Mitch, W.A., 2012. Halonitroalkanes, halonitriles, haloamides, and Nnitrosamines: a critical review of nitrogenous disinfection byproduct formation pathways. Environ Sci Technol 46 (1), 119-131.

Sharpless, C.M., Linden, K.G., 2003. Experimental and Model Comparisons of Lowand Medium-Pressure Hg Lamps for the Direct and H2O2 Assisted UV Photodegradation of N-Nitrosodimethylamine in Simulated Drinking Water. Environmental Science \& Technology 37 (9), 1933-1940. 
Simard, S., Tardif, R., Rodriguez, M.J., 2013. Variability of chlorination by-product occurrence in water of indoor and outdoor swimming pools. Water Res 47 (5), 1763-1772.

Simon, A., McDonald, J.A., Khan, S.J., Price, W.E., Nghiem, L.D., 2013. Effects of caustic cleaning on pore size of nanofiltration membranes and their rejection of trace organic chemicals. Journal of Membrane Science 447 (0), 153-162.

Singapore, S.L., 2014. Happy Fish Swim School-Public Swimming Pools in Singapore.

Sohn, J., Amy, G., Cho, J., Lee, Y., Yoon, Y., 2004. Disinfectant decay and disinfection by-products formation model development: chlorination and ozonation by-products. Water Res 38 (10), 2461-2478.

Spain, 2007. Swimming Pool Regulations. https://www.eyeonspain.com/spainmagazine/pool-legals.aspx.

Spangenberg, D., Möller, U., Kleinermanns, K., 1996. Photooxidation of exhaust pollutants IV. Photocatalytic and thermal decomposition of trichloroacetic acid: degradation efficiencies and products. Chemosphere 33 (1), 43-49.

Stack, M.A., Fitzgerald, G., O'Connell, S., James, K.J., 2000. Measurement of trihalomethanes in potable and recreational waters using solid phase micro extraction with gas chromatography-mass spectrometry. Chemosphere 41 (11), 1821-1826.

Stottmeister, E., Naglitsch, F., 1996. Human exposure to other disinfection byproducts than trihalomethanes in swimming pools. Annual report of the Federal Environmental Agency, Berlin, Germany (in German).

Stumm, W., Morgan, J.J. (2012) Aquatic chemistry: chemical equilibria and rates in natural waters, John Wiley \& Sons.

Su, M., Wang, D.-X., Wang, X.-L., Ando, M., Shintani, T., 2006. Rejection of ions by $\mathrm{NF}$ membranes for binary electrolyte solutions of $\mathrm{NaCl}, \mathrm{NaNO} 3, \mathrm{CaCl} 2$ and $\mathrm{Ca}(\mathrm{NO} 3) 2$. Desalination 191 (1-3), 303-308.

Sutzkover, I., Hasson, D., Semiat, R., 2000. Simple technique for measuring the concentration polarization level in a reverse osmosis system. Desalination $131(1), 117-127$.

Tang, C. (2006) Effect of flux and feedwater composition on fouling of reverse osmosis and nanofiltration membranes by humic acid, ProQuest.

Tang, C.Y., Chong, T.H., Fane, A.G., 2011. Colloidal interactions and fouling of NF and RO membranes: a review. Advances in Colloid and Interface Science 164 (1-2), 126-143.

Tang, C.Y., Fu, Q.S., Robertson, A.P., Criddle, C.S., Leckie, J.O., 2006. Use of Reverse Osmosis Membranes to Remove Perfluorooctane Sulfonate (PFOS) from Semiconductor Wastewaterâ€ Environmental Science \& Technology 40 (23), 7343-7349.

Tang, C.Y., Kwon, Y.-N., Leckie, J.O., 2007. Fouling of reverse osmosis and nanofiltration membranes by humic acid-effects of solution composition and hydrodynamic conditions. Journal of Membrane Science 290 (1), 86-94.

Tang, C.Y., Kwon, Y.-N., Leckie, J.O., 2009a. Effect of membrane chemistry and coating layer on physiochemical properties of thin film composite polyamide 
RO and NF membranes: I. FTIR and XPS characterization of polyamide and coating layer chemistry. Desalination 242 (1), 149-167.

Tang, C.Y., Kwon, Y.-N., Leckie, J.O., 2009b. Effect of membrane chemistry and coating layer on physiochemical properties of thin film composite polyamide RO and NF membranes: II. Membrane physiochemical properties and their dependence on polyamide and coating layers. Desalination 242 (1), 168-182.

Tang, S., Wang, X.M., Yang, H.W., Xie, Y.F.F., 2013. Haloacetic acid removal by sequential zero-valent iron reduction and biologically active carbon degradation. Chemosphere 90 (4), 1563-1567.

Teo, T.L.L., Coleman, H.M., Khan, S.J., 2015. Chemical contaminants in swimming pools: Occurrence, implications and control. Environment international 76, 16-31.

Terasaki, M., Makino, M., 2008. Determination of chlorinated by-products of parabens in swimming pool water. International Journal of Environmental Analytical Chemistry 88 (13), 911-922.

Tilstam, U., Weinmann, H., 2002. Trichloroisocyanuric acid: A safe and efficient oxidant. Organic process research \& development 6 (4), 384-393.

Tu, K., Nghiem, L., Chivas, A., 2011. Coupling effects of feed solution pH and ionic strength on the rejection of boron by $\mathrm{NF} / \mathrm{RO}$ membranes. Chemical Engineering Journal 168 (2), 700-706.

Tung, H.H., Xie, Y.F., 2009. Association between haloacetic acid degradation and heterotrophic bacteria in water distribution systems. Water Res 43 (4), 971 978.

Uyak, V., Koyuncu, I., Oktem, I., Cakmakci, M., Toroz, I., 2008. Removal of trihalomethanes from drinking water by nanofiltration membranes. Journal of Hazardous materials 152 (2), 789-794.

van den Brink, P., Zwijnenburg, A., Smith, G., Temmink, H., van Loosdrecht, M., 2009. Effect of free calcium concentration and ionic strength on alginate fouling in cross-flow membrane filtration. Journal of Membrane Science 345 (1-2), 207-216.

Verliefde, A.R.D., Cornelissen, E.R., Heijman, S.G.J., Verberk, J.Q.J.C., Amy, G.L., Van der Bruggen, B., van Dijk, J.C., 2008. The role of electrostatic interactions on the rejection of organic solutes in aqueous solutions with nanofiltration. Journal of Membrane Science 322 (1), 52-66.

Vidal, L., Chisvert, A., Canals, A., Salvador, A., 2010. Ionic liquid-based singledrop microextraction followed by liquid chromatography-ultraviolet spectrophotometry detection to determine typical UV filters in surface water samples. Talanta 81 (1-2), 549-555.

Villanueva, C., Font Ribera, L., 2012. Health impact of disinfection by-products in swimming pools. Annali dell'Istituto superiore di sanità 48 (4), 387-396.

Villanueva, C.M., Cantor, K.P., Grimalt, J.O., Castaño-Vinyals, G., Malats, N., Silverman, D., Tardon, A., Garcia-Closas, R., Serra, C., Carrato, A., 2006. Assessment of lifetime exposure to trihalomethanes through different routes. Occupational and environmental medicine 63 (4), 273-277.

Villanueva, C.M., Cantor, K.P., Grimalt, J.O., Malats, N., Silverman, D., Tardon, A., Garcia-Closas, R., Serra, C., Carrato, A., Castano-Vinyals, G., 2007a. 
Bladder cancer and exposure to water disinfection by-products through ingestion, bathing, showering, and swimming in pools. American journal of epidemiology 165 (2), 148-156.

Villanueva, C.M., Gagniere, B., Monfort, C., Nieuwenhuijsen, M.J., Cordier, S., 2007b. Sources of variability in levels and exposure to trihalomethanes. Environmental research 103 (2), 211-220.

Von Gunten, U., Oliveras, Y., 1998. Advanced oxidation of bromide-containing waters: bromate formation mechanisms. Environ Sci Technol 32 (1), 63-70.

Waller, K., Swan, S.H., DeLorenze, G., Hopkins, B., 1998. Trihalomethanes in drinking water and spontaneous abortion. Epidemiology 9 (2), 134-140.

Wang, G.-S., Hsieh, S.-T., Hong, C.-S., 2000. Destruction of humic acid in water by UV light — catalyzed oxidation with hydrogen peroxide. Water Research 34 (15), 3882-3887.

Wang, W., Qian, Y., Boyd, J.M., Wu, M., Hrudey, S.E., Li, X.F., 2013. Halobenzoquinones in swimming pool waters and their formation from personal care products. Environmental Science and Technology 47 (7), 32753282.

Wang, X., Leal, M.G., Zhang, X., Yang, H., Xie, Y., 2014. Haloacetic acids in swimming pool and spa water in the United States and China. Frontiers of Environmental Science \& Engineering, 1-5.

Watson, K., Farré, M.J., Birt, J., McGree, J., Knight, N., 2015. Predictive models for water sources with high susceptibility for bromine-containing disinfection by-product formation: implications for water treatment. Environmental Science and Pollution Research 22 (3), 1963-1978.

Weaver, W.A., Li, J., Wen, Y., Johnston, J., Blatchley, M.R., Blatchley, E.R., 3rd, 2009. Volatile disinfection by-product analysis from chlorinated indoor swimming pools. Water Res 43 (13), 3308-3318.

Weng, S., Blatchley, E.R., 3rd, 2011. Disinfection by-product dynamics in a chlorinated, indoor swimming pool under conditions of heavy use: national swimming competition. Water Res 45 (16), 5241-5248.

Weng, S., Li, J., Blatchley Iii, E.R., 2012. Effects of UV254 irradiation on residual chlorine and DBPs in chlorination of model organic-N precursors in swimming pools. Water Research 46 (8), 2674-2682.

Weng, S., Sun, P., Ben, W., Huang, C.-H., Lee, L.T., Blatchley Iii, E.R., 2014. The Presence of Pharmaceuticals and Personal Care Products in Swimming Pools. Environmental Science \& Technology Letters 1 (12), 495-498.

Werber, J.R., Osuji, C.O., Elimelech, M., 2016. Materials for next-generation desalination and water purification membranes. Nature Reviews Materials 1, 16018.

White, G., 1999. Handbook of chlorination and alternative disinfectants, 1999, New York: John Wiley and Sons.

WHO, 2006. Guidelines for Safe Recreational Water Environments. Volume 2: Swimming Pools and Similar Environments.

WHO, 2008. Guidelines for drinking-water quality - Volume 1: Recommendations, Third edition. 
Wiant, C., 2011. A snapshot of swimmer hygiene behavior. International Journal of Aquatic Research and Education 5 (3), 3.

Wojtowicz, J.A., 1996. Relative bactericidal effectiveness of hypochlorous acid and chloroisocyanurates. Journal of the Swimming Pool and Spa Industry 2 (1), 34-41.

Wojtowicz, J.A., 2004. Effect of cyanuric acid on swimming pool maintenance. Journal of the Swimming Pool and Spa Industry 5 (1), 15-19.

Wu, C., Wei, D., Fan, J., Wang, L., 2001. Photosonochemical degradation of trichloroacetic acid in aqueous solution. Chemosphere 44 (5), 1293-1297.

Xiao, F., Zhang, X., Zhai, H., Lo, I.M., Tipoe, G.L., Yang, M., Pan, Y., Chen, G., 2012. New halogenated disinfection byproducts in swimming pool water and their permeability across skin. Environ Sci Technol 46 (13), 7112-7119.

Xiao, Y., Fan, R., Zhang, L., Yue, J., Webster, R.D., Lim, T.-T., 2014. Photodegradation of iodinated trihalomethanes in aqueous solution by UV 254 irradiation. Water Res 49, 275-285.

Xu, B., Chen, Z., Qi, F., Ma, J., Wu, F., 2009. Rapid degradation of new disinfection by-products in drinking water by UV irradiation: N-Nitrosopyrrolidine and N-nitrosopiperidine. Separation and Purification Technology 69 (1), 126-133.

Xu, P., Drewes, J.E., Bellona, C., Amy, G., Kim, T.-U., Adam, M., Heberer, T., 2005. Rejection of emerging organic micropollutants in nanofiltration-reverse osmosis membrane applications. Water Environment Research 77 (1), 40-48.

Xu, X., Mariano, T.M., Laskin, J.D., Weisel, C.P., 2002. Percutaneous Absorption of Trihalomethanes, Haloacetic Acids, and Haloketones. Toxicology and Applied Pharmacology 184 (1), 19-26.

Yang, L., Schmalz, C., Zhou, J., Zwiener, C., Chang, V.W.C., Ge, L., Wan, M.P., 2016. An insight of disinfection by-product (DBP) formation by alternative disinfectants for swimming pool disinfection under tropical conditions. Water Research 101, 535-546.

Yangali-Quintanilla, V., Sadmani, A., McConville, M., Kennedy, M., Amy, G., 2010. A QSAR model for predicting rejection of emerging contaminants (pharmaceuticals, endocrine disruptors) by nanofiltration membranes. Water Research 44 (2), 373-384.

Yeh, R.Y.L., Farré, M.J., Stalter, D., Tang, J.Y.M., Molendijk, J., Escher, B.I., 2014. Bioanalytical and chemical evaluation of disinfection by-products in swimming pool water. Water Research 59, 172-184.

Yoon, S.-H., Lee, C.-H., Kim, K.-J., Fane, A.G., 1998. Effect of calcium ion on the fouling of nanofilter by humic acid in drinking water production. Water Research 32 (7), 2180-2186.

Yoon, Y., Amy, G., Yoon, J., 2005. Effect of $\mathrm{pH}$ and conductivity on hindered diffusion of perchlorate ions during transport through negatively charged nanofiltration and ultrafiltration membranes. Desalination 177 (1-3), 217227.

Zhang, J., She, Q., Chang, V.W., Tang, C.Y., Webster, R.D., 2014. Mining nutrients $(\mathrm{N}, \mathrm{K}, \mathrm{P})$ from urban source-separated urine by forward osmosis dewatering. Environmental Science \& Technology 48 (6), 3386-3394. 
Zhang, P., LaPara, T.M., Goslan, E.H., Xie, Y., Parsons, S.A., Hozalski, R.M., 2009. Biodegradation of haloacetic acids by bacterial isolates and enrichment cultures from drinking water systems. Environmental Science \& Technology 43 (9), 3169-3175.

Zhang, X., Minear, R.A., 2002. Decomposition of trihaloacetic acids and formation of the corresponding trihalomethanes in drinking water. Water Research 36 (14), 3665-3673.

Zhang, Z., Matson, J.V., 1989. Organic Halogen Stabilizers-Mechanisms and Disinfection Efficiencies. Technical Paper Number TP-89-05, 1989 Annual Meeting, Cooling Tower Institute.

Zhao, C., Zhang, J., He, G., Wang, T., Hou, D., Luan, Z., 2013. Perfluorooctane sulfonate removal by nanofiltration membrane the role of calcium ions. Chemical Engineering Journal 233, 224-232.

Zwiener, C., Richardson, S.D., De Marini, D.M., Grummt, T., Glauner, T., Frimmel, F.H., 2007. Drowning in disinfection byproducts? Assessing swimming pool water. Environmental Science \& Technology 41 (2), 363-372. 


\section{List of Publications}

1. Yang, L., Schmalz, C., Zhou, J., Zwiener, C., Chang, V.W.C., Ge, L., Wan, M.P., 2016. An insight of disinfection by-product (DBP) formation by alternative disinfectants for swimming pool disinfection under tropical conditions. Water Research 101, 535-546.

2. Yang, L., She, Q., Wan, M.P., Wang, R., Chang, V.W.C., Tang, C.Y., 2017. Removal of haloacetic acids from swimming pool water by reverse osmosis and nanofiltration. Water Research 116, 116-125.

3. Yang, L., Zhou, J., She, Q., Wan, M.P., Wang, R., Chang, V.W.C., Tang, C.Y., 2017. Role of calcium ions on the removal of haloacetic acids from swimming pool water by nanofiltration: mechanisms and implications. Water Research 110, 332-341.

4. Yang, L., Giannis, A., Chang, V.W.C., Liu, B., Zhang, J., Wang, J.-Y., 2015. Application of hydroponic systems for the treatment of source-separated human urine. Ecological Engineering 81 (0), 182-191.

5. Zhou, J., Fang, W., Cao, Q., Yang, L., Chang, V.W.C., Nazaroff, W., 2016. Influence of moisturizer and relative humidity on human emissions of fluorescent biological aerosol particles. Indoor Air (accepted). 